San Jose State University

SJSU ScholarWorks

Master's Theses

Master's Theses and Graduate Research

Summer 2019

\title{
Synthesis and Evaluation of Novel Silica Hydride-based Stationary Phases for Bioanalytical Applications
}

Seiichiro Watanabe

San Jose State University

Follow this and additional works at: https://scholarworks.sjsu.edu/etd_theses

\section{Recommended Citation}

Watanabe, Seiichiro, "Synthesis and Evaluation of Novel Silica Hydride-based Stationary Phases for Bioanalytical Applications" (2019). Master's Theses. 5050.

DOI: https://doi.org/10.31979/etd.sww8-2×32

https://scholarworks.sjsu.edu/etd_theses/5050

This Thesis is brought to you for free and open access by the Master's Theses and Graduate Research at SJSU ScholarWorks. It has been accepted for inclusion in Master's Theses by an authorized administrator of SJSU ScholarWorks. For more information, please contact scholarworks@sjsu.edu. 
SYNTHESIS AND EVALUATION OF NOVEL SILICA HYDRIDE-BASED STATIONARY PHASES FOR BIOANALYTICAL APPLICATIONS

\author{
A Thesis \\ Presented to \\ The Faculty of the Department of Chemistry \\ San José State University \\ In Partial Fulfillment \\ of the Requirements for the Degree \\ Master of Science
}

by

Seiichiro Watanabe

August 2019 
(C) 2019

Seiichiro Watanabe

ALL RIGHTS RESERVED 
The Designated Thesis Committee Approves the Thesis Titled

SYNTHESIS AND EVALUATION OF NOVEL SILICA HYDRIDE-BASED

STATIONARY PHASES FOR BIOANALYTICAL APPLICATIONS

By

Seiichiro Watanabe

APPROVED FOR THE DEPARTMENT OF CHEMISTRY

SAN JOSÉ STATE UNIVERSITY

August 2019

Joseph J. Pesek, Ph. D.

Department of Chemistry

Roger H. Terrill, Ph. D.

Department of Chemistry

Ningkun Wang, Ph. D.

Department of Chemistry 


\title{
ABSTRACT \\ SYNTHESIS AND EVALUATION OF NOVEL SILICA HYDRIDE-BASED STATIONARY PHASES FOR BIOANALYTICAL APPLICATIONS
}

\author{
by Seiichiro Watanabe
}

Most HPLC columns are packed with silanol (Si-OH)-rich type-B silica that often participates in undesired electrostatic interactions with sample solutes and produces a poor analyte peak shape. These silanols are also known to facilitate the surface hydration required for HILIC mode to retain polar analytes. However, the hydrated surface composition can easily fluctuate, which results in poor reproducibility and requires a lengthy equilibration step. In this study, four novel stationary phases have been developed by using the TYPE-C ${ }^{\text {TM }}$ silica material, in which up to $95 \%$ of the surface silanols have been replaced with $\mathrm{Si}-\mathrm{H}$ group. One of the advantages of this material is the ability to retain polar molecules by operating in adsorption-based ANP mode, despite surface coverage by the non-polar Si-H group. Overall, TYPE-CTM materials eliminate most of the drawbacks observed in Type-B silica. The objectives of this study were three-fold: (1) to develop novel hydride-based columns by surface modification, (2) to evaluate the general chromatographic performances and analyte selectivity by HPLC and ESI-TOF-MS instrumentation, and (3) to explore new bioanalytical applications of the novel stationary phases and ANP chromatography. Here, practical applications of the columns were demonstrated across several research projects: the analysis of phytocannabinoids along with other psychoactive-substances; metabolomics characterization of the USDA grape cultivars; and ANP-coupled HDX-MS method development which may serve as a potential approach to reduce proton back-exchange during structural proteomics assay. 


\section{ACKNOWLEDGMENTS}

First and foremost, I would like to show my sincere gratitude to Dr. Joseph Pesek and Dr. Maria Matyska-Pesek for providing me an opportunity to work in the research group and for being great mentors. I truly appreciate their guidance and valuable feedback throughout the bachelor's and master's program. I would also like to thank Dr. Roger Terrill for dedicating his valuable time as one of my thesis committee members and for willingness to share his knowledge. Many thanks to Dr. Ningkun Wang for also being my committee member and for allowing me to conduct collaborative research. I would like to give special thanks to my research collaborators: Dr. Abraham Wolcott (San Jose State University), Dr. Gary Takeoka (USDA), Dr. Craig Ledbetter (USDA), Dr. Markus Kramer (University of Tübingen), Mr. Josh Young (MicroSolv), and Ms. Tanya Hiltz (MicroSolv). I also would like to acknowledge the chemistry department staff members: Mr. Michael Stephens, Mr. Aklilu Kidane, Mr. Stephen Cappelloni, Ms. Irene Lin, Mr. Randy Kirchner, and Mr. John Hawk for providing technical support and for maintaining the safe laboratory environment.

Lastly, I am truly grateful for my parents, my girlfriend Theresa, and friends for their endless support, encouragement, and patience they showed me over the past two years. I cannot thank you all enough. 


\section{TABLE OF CONTENTS}

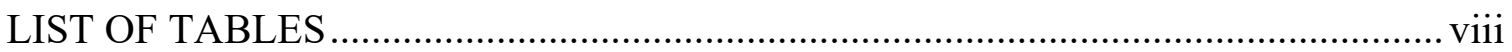

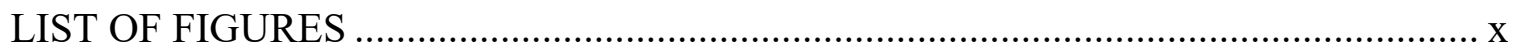

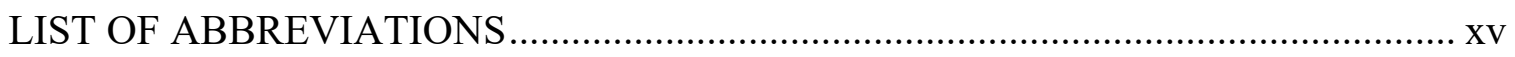

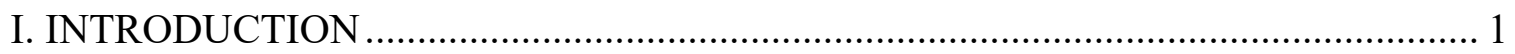

1.1 Background of Chromatography......................................................................... 1

1.2 High-performance Liquid Chromatography (HPLC) …………............................ 2

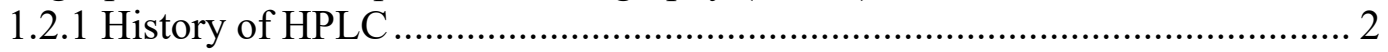

1.2.2 Chromatographic Performance and Data Variables .................................... 3

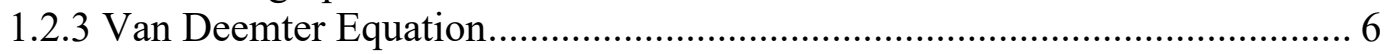

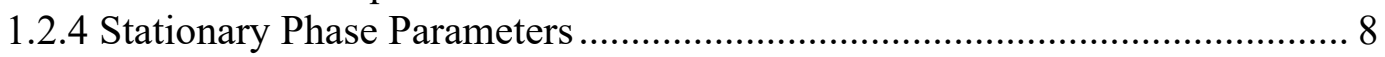

1.3 Modes of Separation ..................................................................................... 10

1.3.1 Organic Normal Phase (ONP) ……...................................................... 10

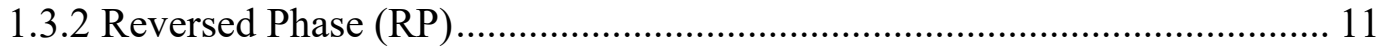

1.3.3 Hydrophilic-interaction Liquid Chromatography (HILIC).......................... 12

1.3.4 Aqueous Normal Phase (ANP) .............................................................. 13

1.4 Column Packing Materials............................................................................... 14

1.4.1 Evolution of Silica Materials .................................................................... 14

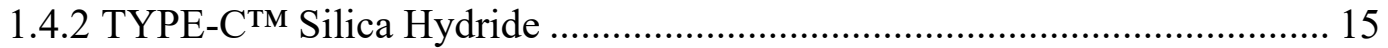

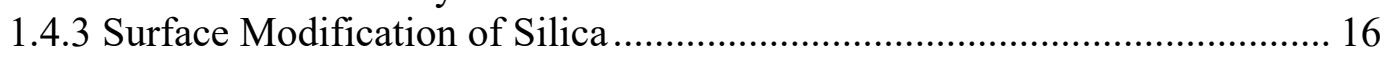

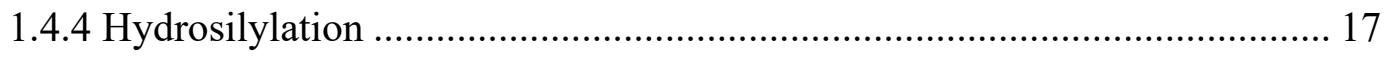

1.5 Characteristics of TYPE-C ${ }^{\text {TM }}$ Silica Columns ...................................................... 19

1.5.1 Surface Properties of Silica Hydride......................................................... 19

1.5.2 Polar Analyte Retention by ANP Chromatography ..................................... 21

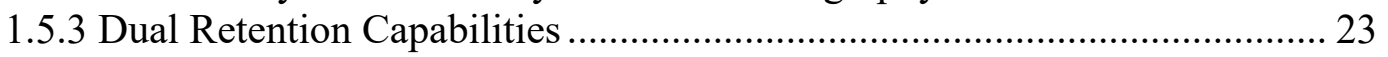

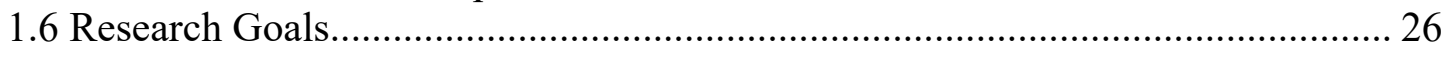

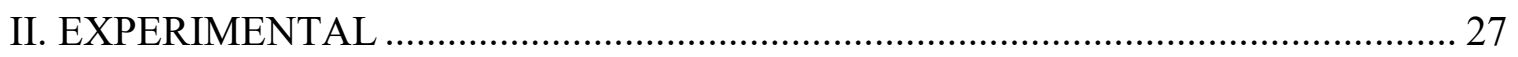

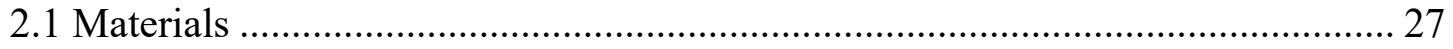

2.1.1 Preparation of the Bonded Phases............................................................... 27

2.1.2 Column Performance Evaluation.................................................................... 29

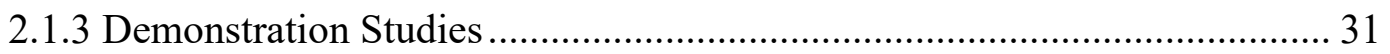

2.1.3.1 Detection of PCBNs in Formulation and Cannabis Plant Extracts........... 31

2.1.3.2 Retention Profile of Controlled Substances .............................................. 32

2.1.3.3 Metabolomics Characterization of USDA Grape Cultivars...................... 32

2.1.3.4 Proton Back-exchange Reduction by ANP-coupled HDX-MS ................ 33

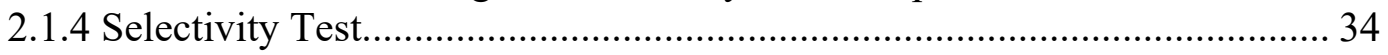

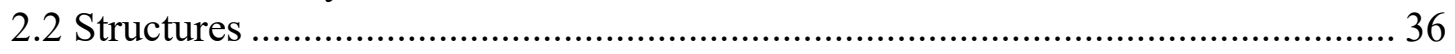

2.2.1 Organic Moieties Used for Surface Modification...................................... 36

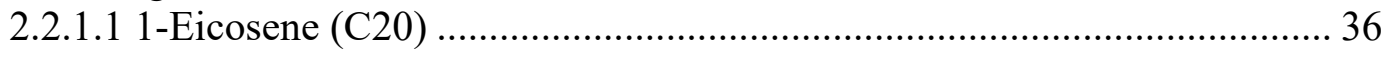

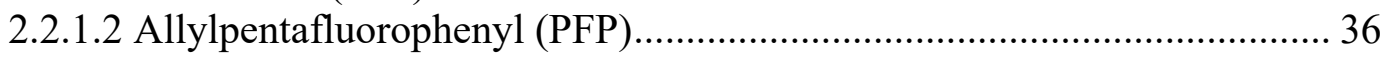




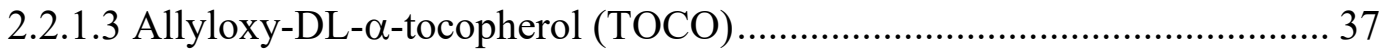

2.2.1.4 10-undecenyloxycarbonyl-leucine (LEU) ................................................ 38

2.2.2 Analytes Used for Column Evaluation ...................................................... 39

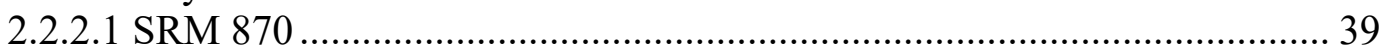

2.2.2.2 Neue Test ....................................................................................... 40

2.2.2.3 Dimethoxybenzene (DMB) Selectivity.................................................... 40

2.2.2.4 Dual Retention Capabilities .................................................................... 41

2.2.3 Analytes Detected in Demonstration Studies ............................................ 42

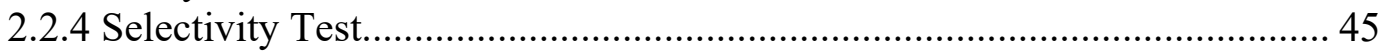

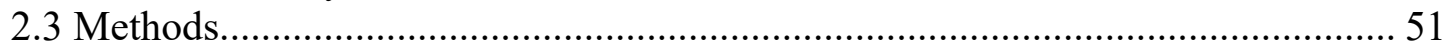

2.3.1 Molecular Dynamics (MD) - Hydride Surface Simulations by MMFF94.. 51

2.3.2 Synthetic Procedures - Hydrosilylation....................................................... 53

2.3.3 Detection of PCBNs in Formulation and Cannabis Plant Extracts.............. 55

2.3.4 Retention Profile of Controlled Substances.................................................... 56

2.3.5 Metabolomics Characterization of USDA Grape Cultivars.......................... 58

2.3.6 Proton Back-exchange Reduction by ANP-coupled HDX-MS .................... 59

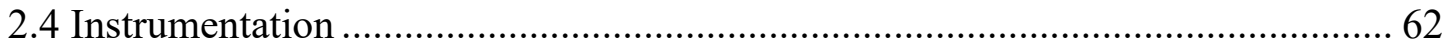

2.4.1 Attenuated Total Reflectance (ATR) ........................................................ 63

2.4.2 Diffuse Reflectance Infrared Fourier Transform Spectroscopy (DRIFTS) . 63

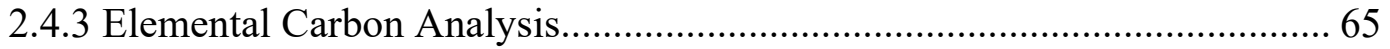

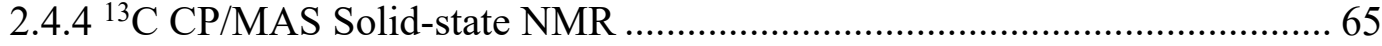

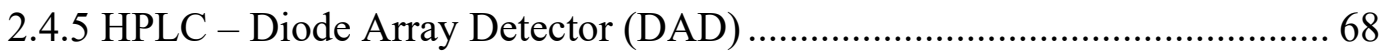

2.4.6 LC/MS - Electrospray Ionization Time-of-flight (ESI-TOF-MS) .............. 70

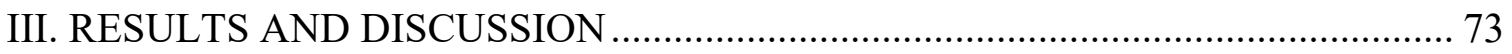

3.1 MD Simulations of the Hydride Surface ……………….................................... 73

3.2 Hydrosilylation Products ................................................................................. 74

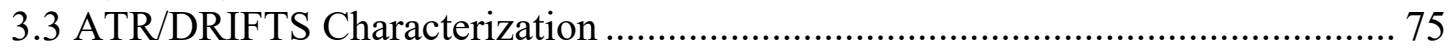

3.4 Elemental Carbon Analysis - Surface Coverage ................................................ 82

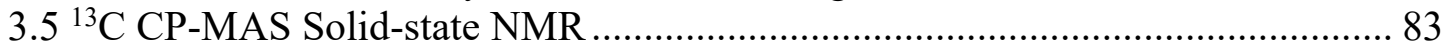

3.6 Column Performance Evaluation by HPLC-DAD ………………………............. 87

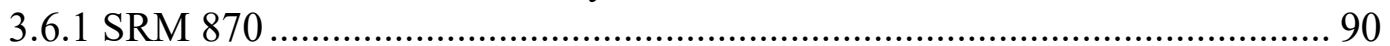

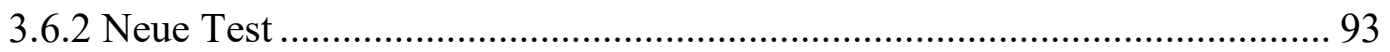

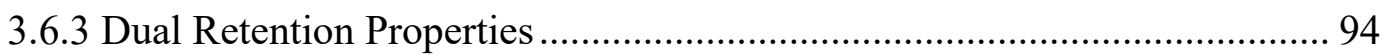

3.6.4 Dimethoxybenzene Selectivity .................................................................. 96

3.7 Detection of PCBNs in Formulation and Cannabis Plant Extracts....................... 99

3.8 Retention Profile of Controlled substances....................................................... 102

3.9 Metabolomics Characterization of USDA Grape Cultivars................................. 106

3.10 Proton Back-exchange Reduction by ANP-coupled HDX-MS ......................... 118

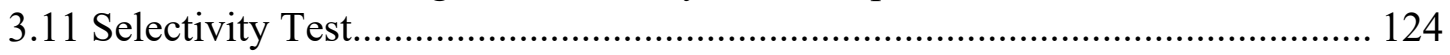

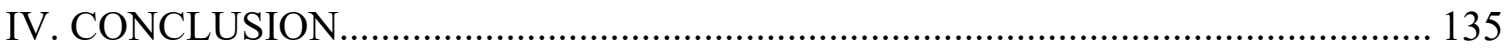

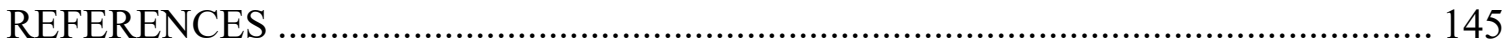




\section{LIST OF TABLES}

Table 1. Reagents Used for Hydrosilylation............................................................... 28

Table 2. Miscellaneous Equipment Used During Synthesis............................................ 29

Table 3. Reagents Used for Mobile Phase Preparation....................................................... 30

Table 4. Compounds Analyzed for Column Performance Evaluation. ............................. 30

Table 5. Miscellaneous Materials Used in the Study ........................................................ 31

Table 6. Samples and Reference Standards Used for Cannabinoids Analysis.................. 31

Table 7. List of Materials and Standards Used for Human Urine Analysis. .................... 32

Table 8. List of Standards and Samples Used for the USDA Grape Analysis. ................ 33

Table 9. Materials Used for ANP-HDX-MS Study....................................................... 33

Table 10. List of Analytes Tested for Column Selectivity.............................................. 34

Table 11. Parameters Used for the MD Simulations of Silica in Aqueous Media. .......... 53

Table 12. Amount of Materials Used for Hydrosilylation.............................................. 54

Table 13. List of Instruments Used in This Study ........................................................... 62

Table 14. Summary of Hydrosilylation for the Four Phases. .......................................... 74

Table 15. Carbon Content and Surface Coverage of the Bonded Moieties...................... 83

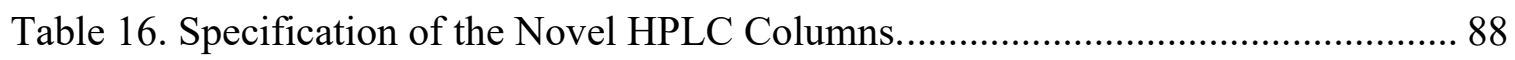

Table 17. Evaluation Criteria in SRM 870 Test and Calculated Column Parameters...... 91

Table 18. Neue Test - Hydrophobicity Parameter Comparison........................................ 94

Table 19. Isotope Mass Distribution of $\mathrm{D}_{2} \mathrm{O}$-EKP Peptide at All Tested Conditions. ... 120

Table 20. A-II Peptide Mass Profile at Normalized Solvent Exposure Time.................. 121

Table 21. Statistical Significance Test for BE Reduction by ANP Gradient.................. 122 
Table 22. A-II Peptide Mass Profile at Normalized Aqueous Solvent Volume. 123

Table 23. Summary of Four Column Properties. 136

Table 24. Analyte Retention Time in Dual Retention Capability Test......................... 136

Table 25. Analyte Retention Time in Evaluation Tests Under RP Condition................ 137

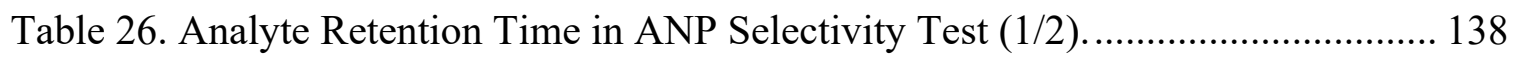

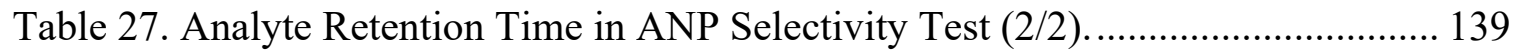

Table 28. Analyte Retention Time in RP Selectivity Test....................................... 140

Table 29. RP Analyte Retention Profile in Bioanalytical Projects. .............................. 141 


\section{LIST OF FIGURES}

Figure 1. Simplified Scheme of a HPLC System. .................................................... 3

Figure 2. Three Fundamental Parameters in Chromatography..................................... 4

Figure 3. Three Terms in Van Deemter Equation........................................................ 6

Figure 4. Key Parameters in HPLC Column. ........................................................... 8

Figure 5. Scheme of Adsorption and Partition-based Retention Mechanism.................. 12

Figure 6. Classification of Chromatography........................................................ 13

Figure 7. Evolution of Chromatographic Silica Materials........................................ 15

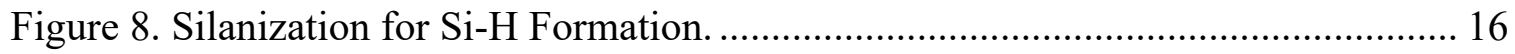

Figure 9. Hydrosilylation for the Production of Bonded Stationary Phase. .................... 17

Figure 10. The Chalk-Harrod Mechanism of Olefin Hydrosilylation. ............................ 18

Figure 11. Comparison of the Two Silica Surfaces................................................... 19

Figure 12. Chromatographic Property of Silica Hydride-based Stationary Phase........... 20

Figure 13. Amount of the Adsorbed Water Monolayer(s) by Two Silica Materials....... 21

Figure 14. Surface Property Differences Among the Two Silica Materials................... 22

Figure 15. Retention of Hydrophilic and Hydrophobic Analytes by a Single Column.... 24

Figure 16. The Analyte Retention Profile of a Silica Hydride-based C18 Column. ........ 25

Figure 17. Retention of Hydrophilic and Hydrophobic Peptides in a Single Run........... 26

Figure 18. Structure of 1-eicosene (C20) Moiety................................................. 36

Figure 19. Structure of Allylpentafluorobenzene (PFP) Moiety. ................................. 37

Figure 20. Structure of Allyloxy-DL- $\alpha$-tocopherol (TOCO) Moiety.............................. 38

Figure 21. Structure of N-undecanoxycarbonyl-leucinate (LEU) Moiety....................... 38 
Figure 22. Five Analytes in SRM 870 Mixture......................................................... 39

Figure 23. Compounds Used in Neue Test. .................................................................. 40

Figure 24. Structures of Anisole and Three Dimethoxybenzenes. ................................... 40

Figure 25. Compounds Used to Evaluate Dual Retention Properties................................. 41

Figure 26. Structures of Standards Used in Grape Metabolomics Analysis..................... 42

Figure 27. Structures of Common Drugs of Abuse. .................................................... 43

Figure 28. Structures of Illicit Substances Detected in Neat Solution.............................. 44

Figure 29. Structures of Phytocannabinoids of Interest................................................... 44

Figure 30. Structures of Two Model Peptides Used in ANP-HDX-MS Study............... 45

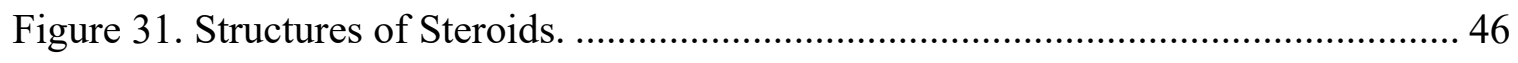

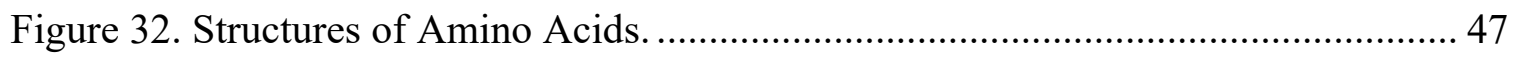

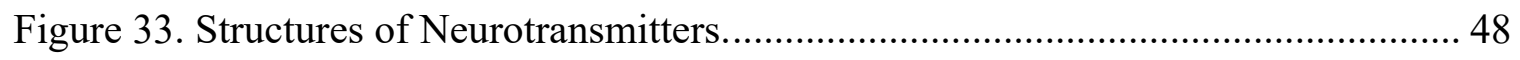

Figure 34. Structures of Nucleobases. ......................................................................... 48

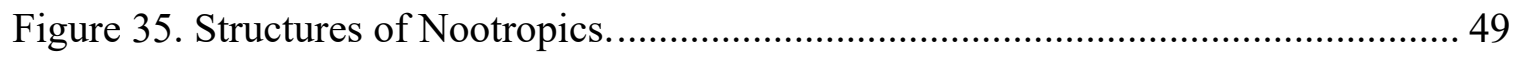

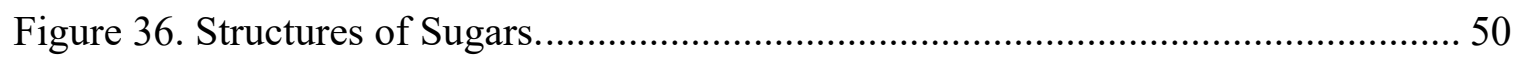

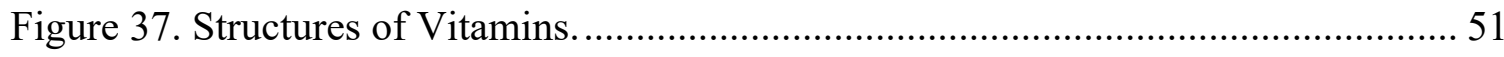

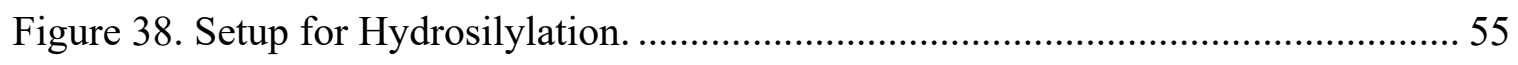

Figure 39. Sample Preparation Workflow for Controlled Substance Analyses................ 57

Figure 40. Scheme for Ordinary HDX-MS (Top) and ANP-HDX Evaluation (Below). . 61

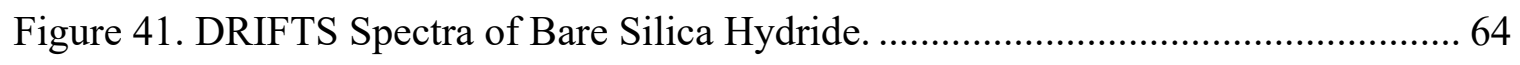

Figure 42. Instrument Used for ${ }^{13} \mathrm{C}$ CP-MAS Solid-state NMR Analysis. ...................... 67

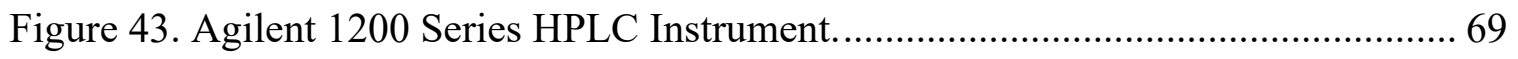


Figure 44. Scheme for UV and PDA Detector. 69

Figure 45. Agilent 1100/1200 Series HPLC Coupled with 6200 Series ESI-TOF-MS.... 70

Figure 46. Scheme for Electrospray Ionization. ................................................... 71

Figure 47. Scheme for Traditional (Top) and Orthogonal-acceleration TOF (Below).... 72

Figure 48. MD Simulations of Ordinary (Top) and Hydride (Bottom) Silica Surfaces. .. 73

Figure 49. The Reacted Bonded Phases. (From left: TOCO, PFP, C20)........................ 74

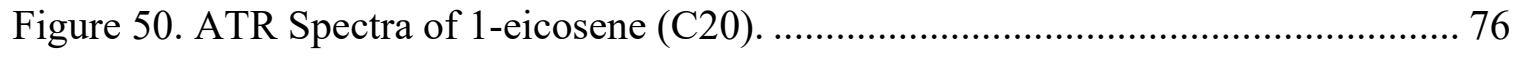

Figure 51. ATR Spectra of Allylpentafluorobenzene (PFP) ...................................... 76

Figure 52. ATR Spectra of Allyloxy-DL-alpha-tocopherol (TOCO)............................ 77

Figure 53. ATR Spectra of 10-undecenyloxycarbonyl-leucine (LEU) .......................... 77

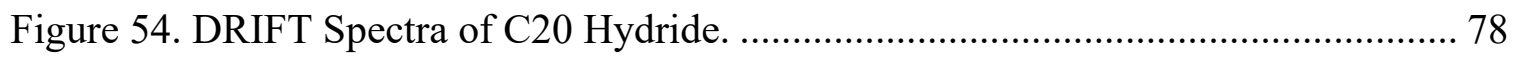

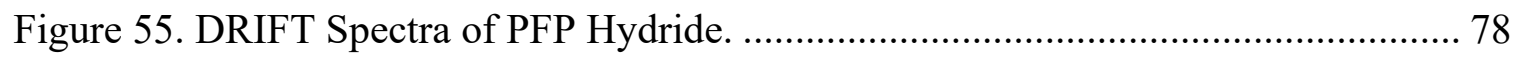

Figure 56. DRIFT Spectra of TOCO Hydride....................................................... 79

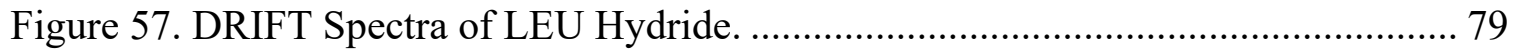

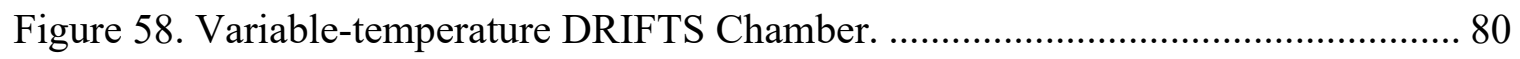

Figure 59. VT-DRIFT Spectra of Two Silica Materials at Room Temperature.............. 81

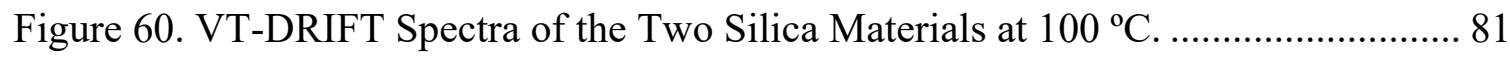

Figure 61. Overlaid VT-DRIFT Spectra of Silica Hydride from $25^{\circ} \mathrm{C}-600^{\circ} \mathrm{C} \ldots \ldots \ldots \ldots . . . . . .82$

Figure $62 .{ }^{13} \mathrm{C}$ CP-MAS Solid-state NMR Spectra of C20 Hydride.............................. 85

Figure $63 .{ }^{13} \mathrm{C}$ CP-MAS Solid-state NMR Spectra of PFP Hydride. ............................ 85

Figure $64 .{ }^{13} \mathrm{C}$ CP-MAS Solid-state NMR Spectra of TOCO Hydride. ........................... 86

Figure $65 .{ }^{13} \mathrm{C}$ CP-MAS Solid-state NMR Spectra of LEU Hydride. ............................ 86 
Figure 66. Newly Packed Columns (From Left: TOCO, PFP, C20, and LEU)............... 87

Figure 67. QC Test Results for TOCO (Left) and PFP (Right)................................ 89

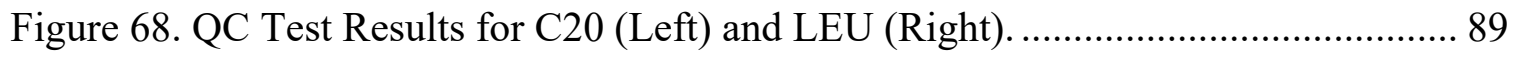

Figure 69. Chromatograms of SRM 870 Mixture Analysis........................................ 91

Figure 70. Calculation for Peak Asymmetric and Tailing Factor................................ 92

Figure 71. Neue Test for Column Hydrophobicity ................................................. 93

Figure 72. Retention Profiles of the Novel Hydride-based Stationary Phases................ 95

Figure 73. DMB Selectivity Evaluation for the Aromatic Columns. ............................ 97

Figure 74. DMB Selectivity Evaluation for All Four Stationary Phases........................ 98

Figure 75. CBD Detected from the SPE-processed Gummy Edible. .......................... 100

Figure 76. Overlaid Chromatograms of Four Cannabis Plant Extracts. ........................ 101

Figure 77. Calibration Curve for CBD Quantification. ......................................... 102

Figure 78. Retention Profiles of Eleven Illicit Substances Detected in Human Urine... 103

Figure 79. Retention Profile of Eight Commonly Abused Drugs in Neat Solution. ...... 105

Figure 80. EIC of Heroin in Neat Solution at Magnified Scale.................................. 106

Figure 81. Retention Profile of Five Flavonoid Reference Standards......................... 107

Figure 82. Catechin and Epicatechin Detected in Five Grape Cultivars. ..................... 109

Figure 83. K3G Detected in Five Grape Cultivars. ................................................. 109

Figure 84. Q3G Detected in Five Grape Cultivars. ............................................ 110

Figure 85. EIC Overlay of Quercetin Standard and Five Grape Sample Injections...... 111

Figure 86. Flavonoid Biosynthetic Pathway..................................................... 112

Figure 87. Flavone and Flavonol Biosynthetic Pathway........................................ 113 
Figure 88. Heatmap Representation of Relative Metabolite Abundance. 114

Figure 89. Metabolome Abundance Differences Between the Two Types of Grapes. .. 116

Figure 90. Metabolome Abundance Differences Between Y539/\#162 vs. \#169. .......... 117

Figure 91. Isotope Mass Distribution of $\mathrm{D}_{2} \mathrm{O}$-EKP at Various Elution Conditions. ...... 118

Figure 92. ANP and RP Elution of $\mathrm{D}_{2} \mathrm{O}-\mathrm{EKP}$ at Normalized $\% \mathrm{H}_{2} \mathrm{O}$ Composition. ...... 119

Figure 93. ANP and RP Elution of A-II at Normalized Retention Time....................... 121

Figure 94. A-II Peptide Elution with ANP/RP Gradient Using PFP Column. ............... 123

Figure 95. RP Retention and Selectivity Profile of Eleven Steroids............................ 125

Figure 96. RP Retention and Selectivity Profile of Eight Nootropic Drugs................... 126

Figure 97. ANP Retention and Selectivity Profile of Eight Nootropic Drugs................ 127

Figure 98. ANP Retention of Four Nucleobases. ..................................................... 128

Figure 99. ANP Retention of Four Neurotransmitters.............................................. 129

Figure 100. RP Retention of Three Vitamins. .......................................................... 131

Figure 101. ANP Retention of Four Vitamins............................................................. 131

Figure 102. ANP Retention of Mono-, Di-, and Tri- Saccharides.................................. 132

Figure 103. ANP Retention of Nineteen Amino Acids (1/2)........................................ 133

Figure 104. ANP Retention of Nineteen Amino Acids (2/2)........................................ 134 


\section{LIST OF ABBREVIATIONS}

\#162 - Y144-162-12 grape cultivar

\#169 - Y144-169-12 grape cultivar

A-II - angiotensin-II

ANP - Aqueous Normal Phase

API - Active Pharmaceutical Ingredients

$\mathrm{A}_{\mathrm{S}}$ - Asymmetric Factor

ATR - Attenuated Total Reflectance

BE - Back-exchange

C20 - 1-eicosene

C33 - C33-30 grape cultivar

CATE - catechin

CBD - cannabidiol

CBDA - cannabidiolic acid

$\mathrm{CP}$ - Cross Polarization

CSA - Chemical Shift Anisotropy

$\mathrm{D}_{2} \mathrm{O}$-EKP $-\mathrm{D}_{2} \mathrm{O}$-reconstituted enkephalin peptides

DAD - Diode Array Detector

DC - Dipolar Coupling

DFR - dihydroflavonol 4-reductase

DHQ - dihydroquercetin

DMB - dimethoxybenzene

$\mathrm{d}_{\mathrm{P}}-$ Particle Diameter

DRIFTS - Diffuse Reflectance Infrared Fourier Transform Spectroscopy

EIC - Extracted Ion Chromatogram

EKC - Electrokinetic Capillary Chromatography

EKP - enkephalin

EPI - epicatechin

ESI - Electrospray Ionization

FTIR - Fourier transform infrared spectroscopy

GC - Gas Chromatography

GDP - Granddaddy Purple (C. indica)

$\mathrm{H}_{2} \mathrm{O}$-EKP $-\mathrm{H}_{2} \mathrm{O}$-reconstituted enkephalin peptides

HDX-MS - Hydrogen-Deuterium Exchange Mass Spectrometry

HETP - Height Equivalent to Theoretical Plate

HILIC - Hydrophilic-Interaction Liquid Chromatography

HPLC - High-Performance (or Pressure) Liquid Chromatography

I.D. - Internal Diameter

IPC - Ion Pair Chromatography

IRE - Internal Reflection Element

IS - Internal Standard

K3G - kaempferol 3-O-glucoside

$\mathrm{L}$ - Length 
LC - Liquid Chromatography

LCY - leucocyanidin

LEU - 10-undecenyloxycarbonyl-leucine

LOD - Limit of Detection

LOQ - Limit of Quantitation

LTM - Late Transition Metal

$\mathrm{M} / \mathrm{Z}$ - Mass-to-Charge Ratio

M3BG - morphine-3-beta-D-glucuronide

M3G - malvidin 3-O-glucoside

MAS - Magic Angle Spinning

MAVEN - Metabolomics and Visualization Engine

MD - Molecular Dynamics

MM - Molecular Mechanics

MMFF94 - Merck Molecular Force Field 94

MS - Mass Spectrometry

NMR - Nuclear Magnetic Resonance

ONP - Organic Normal Phase

PCBNs - phytocannabinoids

PDA - Photo Diode Array

PFP - allylpentafluoro-benzene (or -phenyl)

Q3G - quercetin 3-O-glucoside

QM - Quantum Mechanics

RP - Reversed Phase

RSTGs - Red Seedless Table Grapes

$\mathrm{S} / \mathrm{N}$ - Signal-to-Noise

SEC - Size-Exclusion Chromatography

SFC - Supercritical Fluid Chromatography

SPE - Solid Phase Extraction

SR - Scarlet Royal

SRM 870 - Standard Reference Material 870

SSH - Super Silver Haze (C. sativa)

TES - triethoxysilane

TF - Tailing Factor

THC - tetrahydrocannabinol

THCA - tetrahydrocannabinolic acid

TMCS - trimethylchlorosilane

TOCO - allyloxy-DL-alpha-tocopherol

TOF - Time-of-Flight

TP - Theoretical Plate

UHPLC - Ultra High-Performance (or Pressure) Liquid Chromatography

VD - Van Deemter

VT - Variable-Temperature

WIFI - White Fire OG (Cannabis $s p$. Hybrid)

Y539 - Y539-194-06 grape cultivar 


\section{INTRODUCTION}

\subsection{Background of Chromatography}

Chromatography consists of several techniques that make it possible to separate various molecular species from a complex mixture based on their physical and chemical properties. The term and concept of chromatography was first introduced by RussianItalian botanist Mikhail Tsvet in the early 1900s. The technique was demonstrated in Tsvet's attempt to separate plant pigments such as carotenoids and chlorophylls, by utilizing calcium carbonate as the adsorbent and petroleum ether as the eluent [1]. In chromatography a mixture to be separated is called a sample, consisting of analytes and matrix. Analytes are the molecular species of interest such as chlorophylls and carotenoids; matrix applies to rest of the non-analyte sample components. A sample mixture introduced into the chromatographic system is transported by a stream of mobile phase which travels through a separation medium called a stationary phase. Unique intermolecular interaction(s) between analyte solutes, the stationary phase, and solvent cause each molecular species to travel through the adsorbent at different rates and produces distinct retention times ( $t_{R}$; Section 1.2.2 for more details) [2]. Generally, chromatographic techniques are categorized based on the physical state of the mobile phase: gas (GC), liquid (LC), or supercritical fluid chromatography (SFC), and based on the shape and the physical state of the stationary phase (e.g., thin-layer chromatography, paper chromatography, and column chromatography). Another classification criterion features the mechanism of interaction between the analyte solute and the stationary phase, which will be further discussed in Section 1.3. 


\subsection{High-performance Liquid Chromatography (HPLC)}

\subsubsection{History of HPLC}

Before high-performance liquid chromatography (HPLC) became available, GC was a considerably more powerful technique than LC at the time. Despite GC's high efficiency, some researchers saw little use in GC since the technique is limited to volatile and thermally stable compounds. Thus, separation of many biomolecules had to be done through liquid phase column chromatography. Column LC has many advantages over GC including: compatibility with a broad range of mobile phase solvents; the ability to analyze thermally labile and nonvolatile compounds; and the instrument can be used for sample collection (preparatory application). However, traditional LC primarily depends on the gravitational force to pass the mobile phase through the stationary phase; thus, separation takes hours and sometimes days to complete [3].

Based on Martin and Synge's seminal work in the 1940s [4], a group of researchers predicted that LC can be operated at higher efficiency by drastically reducing the stationary phase particle size and utilizing a high-pressure mechanical pump to increase the mobile phase velocity (Figure 1) [3, 5]. In 1967, the first commercial HPLC instrument, ALC-100, was introduced by Waters Corporation. Over the past fifty years, both HPLC instruments and packing material technology have improved significantly. For instance, an ultra-high-performance liquid chromatography (UHPLC) system can operate at a pressure near 1,000 bar/15,000 psi with a column packed with sub $2 \mu \mathrm{m}$ particles; a nano-HPLC instrument can produce constant flow rates at the nanoliters per minute (nL/min) scale. 


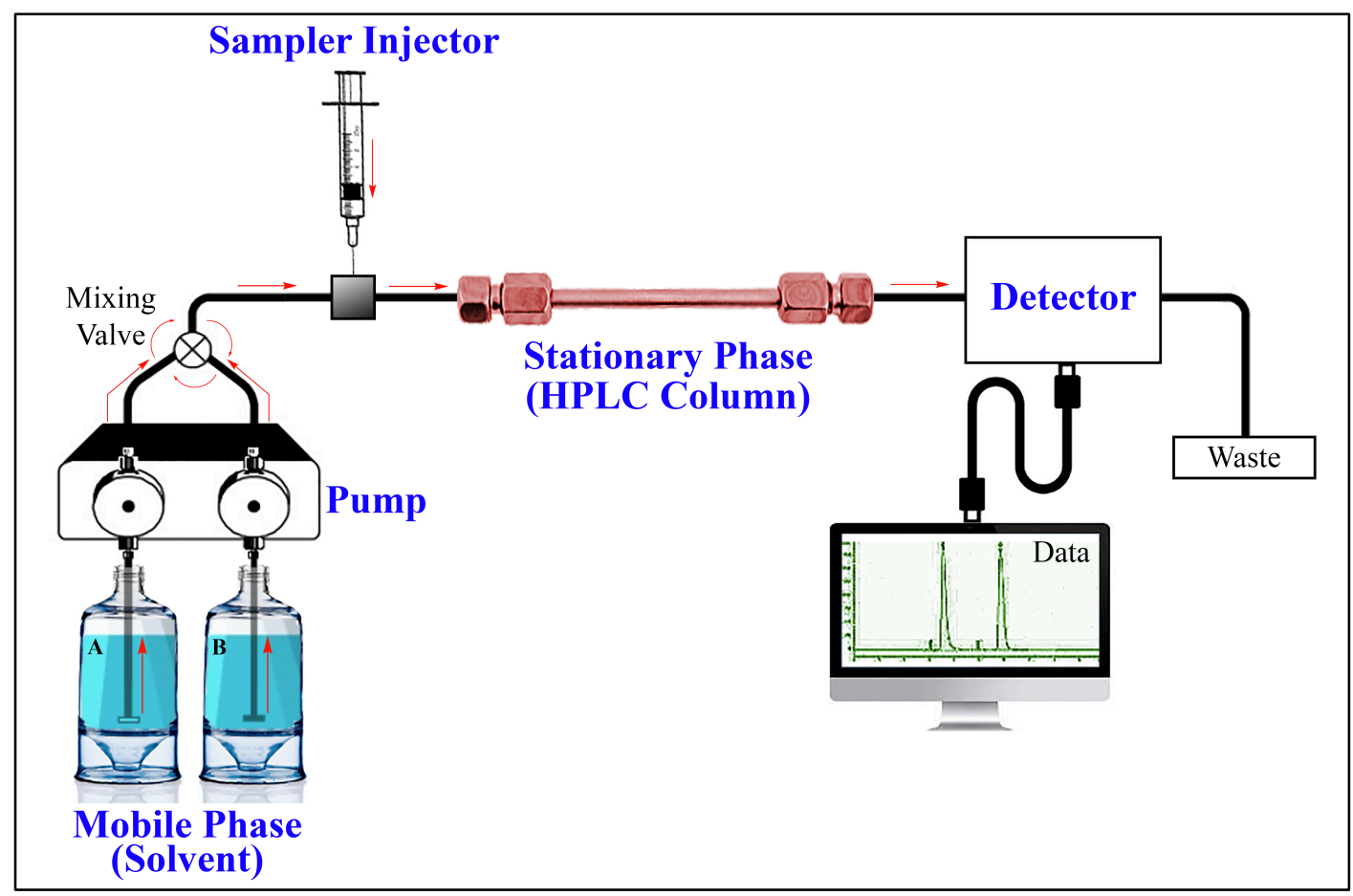

Figure 1. Simplified Scheme of a HPLC System.

In addition, the development of LC-compatible detectors such as electrospray ionization mass spectrometer (ESI-MS) has expanded the functional capabilities of HPLC. Today, HPLC is an essential instrumental analysis technique in numerous fields of research including biopharmaceutical, clinical research, forensic toxicology, food science, and proteomics among others.

\subsubsection{Chromatographic Performance and Data Variables}

In HPLC method development, the general goal is to achieve optimum resolution $\left(\mathrm{R}_{\mathrm{S}}\right)$ in the minimum amount of analysis time. As shown in equation 1.2.2.1, $\mathrm{R}_{\mathrm{S}}$ depends on three components: retention/capacity factor $(k)$, selectivity $(\alpha)$, and column efficiency (N) (Figure 2):

$$
\mathrm{R}_{\mathrm{S}}=\frac{1}{4} \sqrt{\mathrm{N}} \times \frac{(\alpha-1)}{\alpha} \times \frac{\mathrm{k}}{(\mathrm{k}+1)}
$$




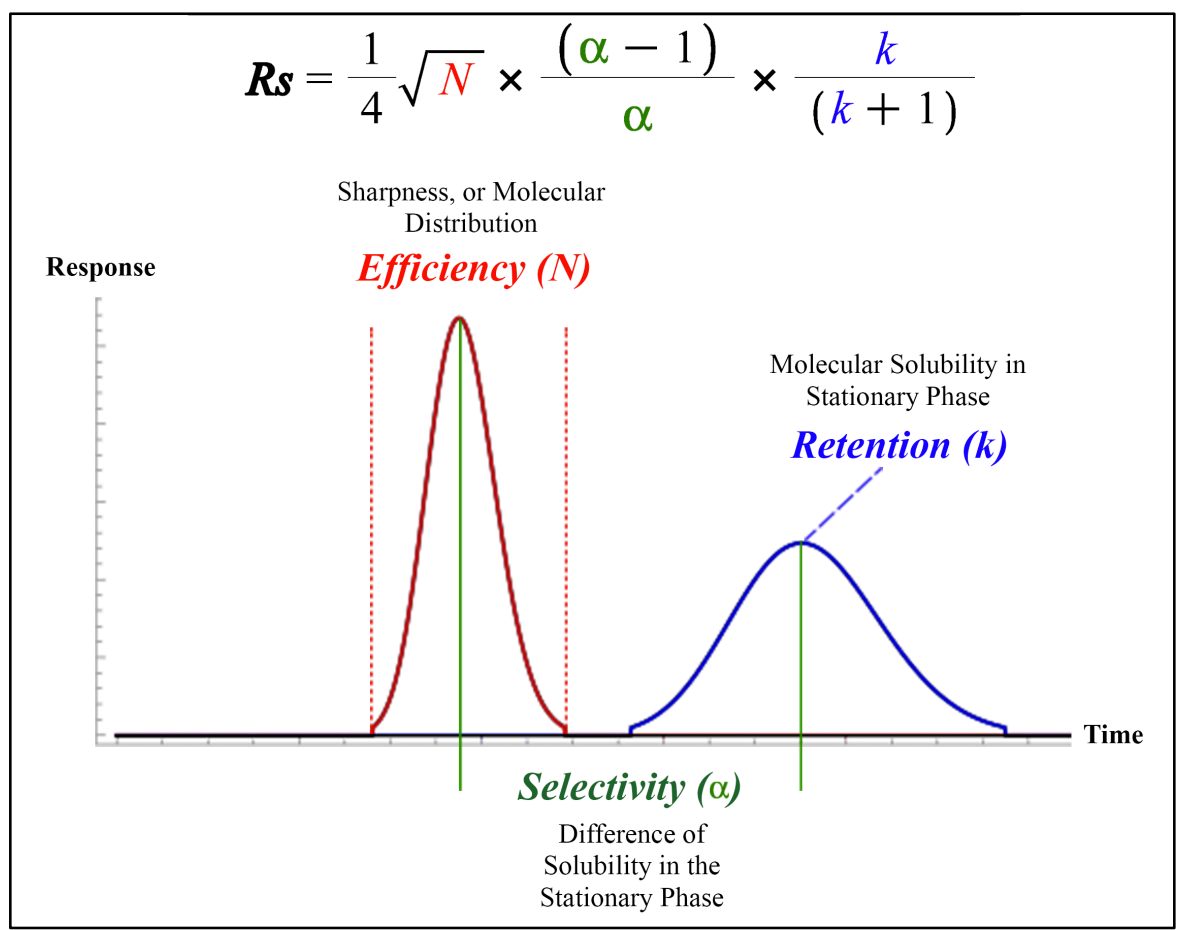

Figure 2. Three Fundamental Parameters in Chromatography.

The resolution value must be higher than 1 to 1.5 in order to ensure the complete separation of sample components so that quantitative information (e.g., peak area and height) can be accurately measured [6].

The retention/capacity factor $\mathrm{k}$ indicates how well an analyte interacts with the stationary phase and is retained $\left(t_{R}\right)$ relative to the retention time of non-retained components $\left(\mathrm{t}_{0}\right)[6]$ :

$$
k=\frac{t_{R}-t_{0}}{t_{0}}
$$

The ratio of the $\mathrm{k}$ factor between the two sample peak apexes is used as a measurement of selectivity $\alpha$, which is the ability of a column to physicochemically distinguish two molecules and elute as far apart as possible from each other (Equation $1.2 .2 .3)[6]$ 


$$
\alpha=\frac{k_{2}}{k_{1}}
$$

Selectivity is one of the major contributors to resolution; however, it does not solely describe how well the two molecules are separated. Even with a large $\alpha$ value, peak broadening due to poor column efficiency $(\mathrm{N})$ could result in a poor separation. The parameter $\mathrm{N}$ is also known as the theoretical plate (TP) number. TP is a concept adopted into chromatography by Martin and Synge, but originally developed for fractional distillation and extraction processes to describe the separation efficiency of the molecules achieved by the system. TP is essentially the hypothetical number of occurrences for an analyte to establish the partition equilibrium with the mobile and stationary phases within a separation media. In other words, TP indicates how frequently the analyte interacts with the stationary phase and achieves mobile-stationary phase equilibrium; therefore, a high "N" value gives narrower peaks. The "N" parameter is calculated using the baseline width $\left(\mathrm{w}_{\mathrm{b}}\right)$ of a peak and its retention time (Eq. 1.2.2.4) [6]:

$$
N=16\left(\frac{t_{R}}{w_{b}}\right)^{2}=5.54\left(\frac{t_{R}}{w_{1 / 2}}\right)^{2}
$$

In some of the literature, the plate number $(\mathrm{N})$ is expressed in another form called the plate height $(\mathrm{H})$, or the height equivalent to theoretical plate (HETP). HETP has also derived from distillation theory and represents the length (or height) of individual theoretical plate, while $\mathrm{N}$ represents the number of theoretical plates present in the given column length (Eq. 1.2.2.5). An efficient separation medium produces a larger $\mathrm{N}$ value; therefore, the HETP value is smaller [6].

$$
\text { HETP }=\frac{L}{N}
$$




\subsubsection{Van Deemter Equation}

Ideally, the peak of a single chemical component should be observed as a sharp straight line. However, when a packed column is used as the stationary phase in chromatography, the analyte peaks are rather described by a Gaussian distribution curve. This is due to several kinetic processes that cause analyte molecules to diffuse across the column and leading to a broader chromatographic peak. The Van Deemter (VD) equation is used to describe the three main causes of band broadening ( $\mathrm{A}, \mathrm{B}$, and $\mathrm{C}$ terms; Figure 3) with respect to column efficiency (HETP) as a function of mobile phase flow rate or linear velocity $(\mathrm{u})($ Eq. 1.2.3.1) [6].

$$
\text { HETP }=A+\frac{B}{u}+\left(C_{S}+C_{M}\right) \cdot u
$$

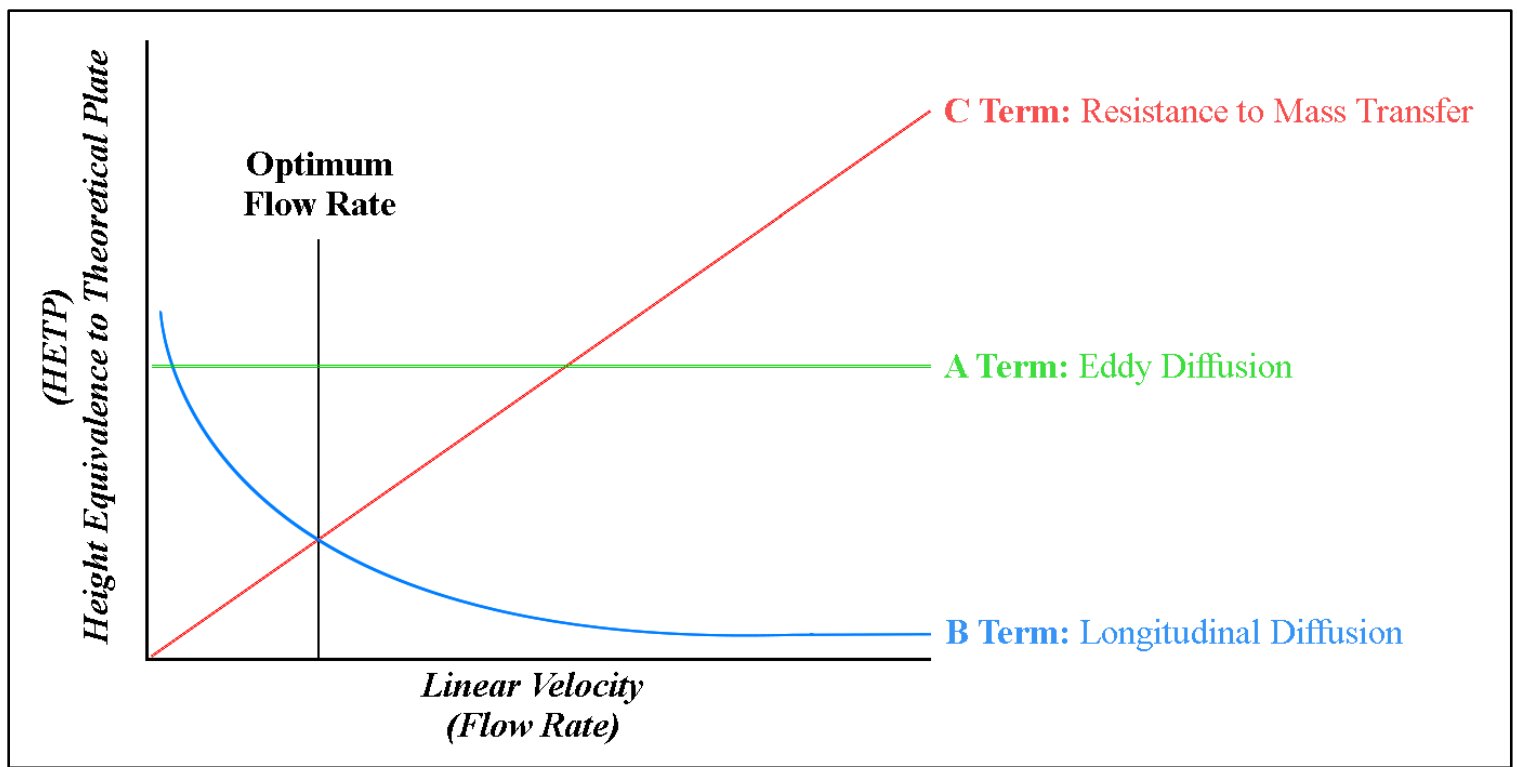

Figure 3. Three Terms in Van Deemter Equation.

The A term in this model accounts for eddy diffusion, which reflects the quality of a packed column. Unlike capillary columns, packed columns create flow channels with various path lengths; therefore, analyte solutes taking different random paths result in 
peak broadening. Eddy diffusion is independent of the mobile phase velocity; however, the effect can be controlled and reduced by utilizing highly uniform and smaller particle packing materials.

The second term, longitudinal diffusion (B) refers to mass dispersion along the longitudinal direction of a column. This phenomenon can be explained by the second law of Fickian diffusion; which is time-dependent, and can be affected by temperature, viscosity, and the size of an analyte molecule. The contribution of longitudinal diffusion to peak broadening in LC is considerably less compared to GC since the diffusion coefficient is 4-5 orders of magnitude lower in liquids than gases [3]. Minimization of this effect can be achieved by increasing the mobile phase flow rate (u) which reduces the total analysis time; therefore, less time is available for the analyte diffusion to occur.

The final $\mathrm{C}$ term is known as the "resistance to mass transfer". Here, mass transfer indicates the transportation of solute from one phase (mobile or stationary) to another. When the mobile phase velocity $(\mathrm{u})$ is higher than the mass transfer coefficient of an analyte, equilibrium between the mobile and the stationary phase would not be established rapidly enough; thereby resulting in peak broadening.

A graphical representation of the VD equation gives a hyperbolic curve when HETP is plotted as a function of linear velocity $(\mathrm{u})$, which allows for optimal flow rate prediction. The equation can be further expanded to calculate the optimal flow rate of a column with a known particle diameter $\left(\mathrm{d}_{\mathrm{p}}\right)$ of the packing material (Eq. 1.2.3.2) [6].

$$
H E T P=A^{\prime} d_{p}+B^{\prime} \frac{D}{u}+C^{\prime} \frac{d_{p}{ }^{2}}{D} \cdot u
$$




\subsubsection{Stationary Phase Parameters}

All the chromatographic data variables mentioned in Section 1.2.2 are affected by, but not limited to, the stationary phase. In HPLC, adsorbent materials are typically packed in stainless-steel housing, also known as a column (Figure 4). Some of the key parameters for the stationary phase includes the column dimensions, which are described by its internal diameter (I.D.) and length (L).

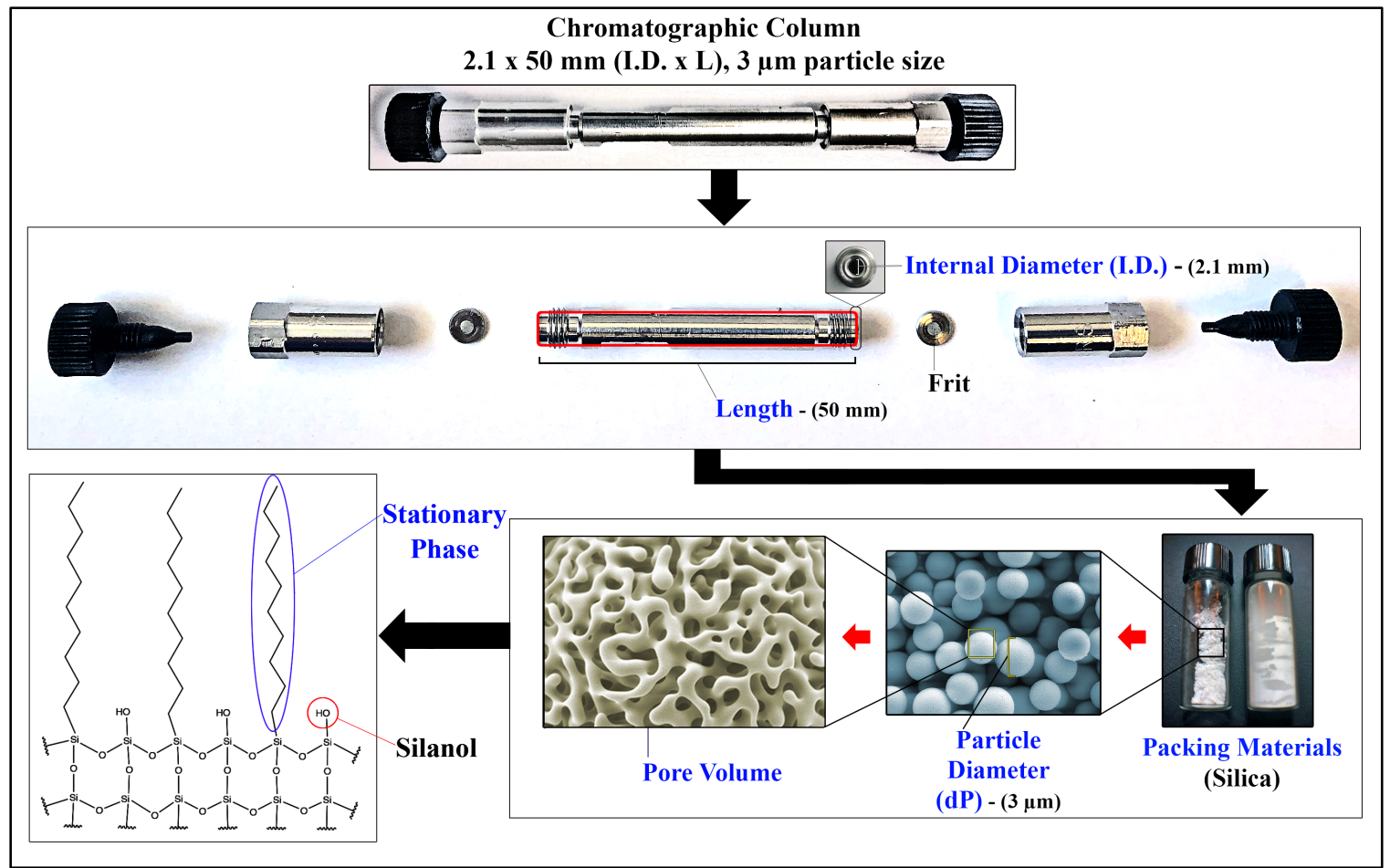

Figure 4. Key Parameters in HPLC Column.

Column I.D. can be scaled down to reduce the flow rate required to achieve maximum efficiency. For instance, a $4.6 \mathrm{~mm}$ I.D. column requires about $1.0 \mathrm{~mL} / \mathrm{min}$ mobile phase velocity while a $2.1 \mathrm{~mm}$ requires $0.3 \mathrm{~mL} / \mathrm{min}$ or less. Therefore, narrower column diameter is more cost effective since a smaller volume of solvents are being used. 
Furthermore, it is beneficial in some detection methods such as electrospray ionization mass spectrometry (ESI-MS) that requires lower mobile phase flow rates.

The length of the column is directly proportional to efficiency. However, when the plate number doubles the resolution increases only by a factor of 1.4. This is likely due to extended elution time enhancing the diffusion effects. Naturally, the downside of the extended column length would be the longer analysis time and increased solvent usage. Column length and internal diameter can together be customized to needs, which could range from: 50 - $300 \mathrm{~mm}$ and $0.1-25 \mathrm{~mm}$, respectively. Generally, 1.0 - $4.6 \mathrm{~mm}$ I.D. is used for analytical applications while preparative columns are $10.0-20.0 \mathrm{~mm}$ or above. Capillary columns have $0.5 \mathrm{~mm}$ or smaller I.D., and it is used when a sensitive detection method is used and/or when the availability of sample material is low [2].

Packing material type, particle diameter $\left(\mathrm{d}_{\mathrm{p}}\right)$, and bonded phase can also significantly influence the quality of chromatographic data as well as the functional capabilities of columns. There are many types of materials used in column chromatography including: Alumina and Titania, among others. Although, the most widely used material is silica due to its cost effectiveness, chemically adjustable surface, and its durability, among other reasons. More details on silica materials will be discussed further in Section 1.4.

Particle diameter $\left(\mathrm{d}_{\mathrm{P}}\right)$ has a significant impact on the column efficiency, primarily by affecting the mass transfer kinetics. By reducing the particle size, one could also expect the reduction in diffusion path lengths, analyte diffusion time, and the time variation in analyte diffusion in/out of the particle pores. Thus, column efficiency is increased due to the reduced peak broadening effect from the $\mathrm{C}$ term in the Van Deemter equation. 
Another factor to consider is the particle size distribution. Narrower size distribution also contributes to improving the column efficiency by reducing the eddy diffusion (A term) which arises from variations in the lengths of flow paths.

Of all the components in a chromatographic column, material surface affects the analyte retention and selectivity the most. For example, in common chromatographic techniques such as reversed-phase and normal-phase, their retention mechanism relies on the adsorption or partitioning of analyte molecules at the silica surface and/or the bonded phase. In contrast, size-exclusion chromatography (SEC) relies on the pore size of the packing material as the critical factor that allows the separation of molecules based on their sizes [2]. Bonded phase refers to the ligand group (organic or metal) chemically attached to the surface of support materials such as silica. Surface modification of the materials gives additional characteristics (e.g., hydrophilicity, hydrophobicity, cationic/anionic, and chiral recognition) to the column, which allows for the unique selectivity and retention of analytes by operating in different modes of separation.

\subsection{Modes of Separation}

\subsubsection{Organic Normal Phase (ONP)}

Normal phase or organic normal phase (ONP) utilizes a polar stationary phase and a nonpolar mobile phase to retain and separate polar molecules. In this technique, hydrophobic molecules elute first while polar molecules are retained the longest. As the name suggests, the mobile phase is consisting only of organic solvents such as hexane, dichloromethane, and ethyl acetate. The stationary phase can be bare silica, where the surface has a large population of polar silanol ( $\mathrm{Si}-\mathrm{OH})$ groups. In addition, 
functionalized silica with polar organic moieties such as amino and cyano can also be used. ONP is a less commonly used technique today and generally considered irreproducible due to difficulties of eliminating residual water content from the mobile phase and the hygroscopic silica surface.

\subsubsection{Reversed Phase (RP)}

Reversed phase (RP) chromatography is the most commonly used technique in HPLC. The retention order of RP is reversed from the ONP chromatography; therefore, it is used for the separation of hydrophobic compounds. Numerous types of RP stationary phases are commercially available, including $\mathrm{C} 18, \mathrm{C} 8$, and phenyl, with $\mathrm{C} 18$ being the most popular form. RP retention is facilitated by hydrophobic interaction and can be explained by either or both adsorption and partition-based mechanisms [7-9].

RP chromatography and its stationary phases can also be used for the analysis of charged-polar compounds when an ionic surfactant is used as a mobile phase additive; this approach is known as ion-pairing chromatography (IPC). Pairing reagents such as alkylammonium cations and alkyl sulfonate anions enhance the retention of a counter ion on a RP column by increasing the paired-analytes' hydrophobicity. IPC can be an effective approach for HPLC-UV detection; however, it is not an ideal choice for mass spectrometry. The use of a pairing reagent not only increases the complexity of operation and interpretation of a mass spectrum, but also exposes the column and instrument to the risk of contamination $[6,10]$. 


\subsubsection{Hydrophilic-interaction Liquid Chromatography (HILIC)}

Hydrophilic-interaction liquid chromatography (HILIC) and ONP are similar in the sense that both techniques are used for polar analyte retention. In HILIC, the mobile phase is a combination of water and water-soluble organic solvents such as methanol and acetonitrile. The fundamental difference between the two modes is the retention mechanism: adsorption for ONP and partition for HILIC. Retention by a partition mechanism is facilitated by the hydration layer formed around the polar surface of the stationary phase. Essentially, the adsorbed water around the surface of silica is acting as an immobilized liquid stationary phase, and analyte partitioning takes place between the water layer and bulk mobile phase (Figure 5). Thus, analyte solutes are separated based on their hydrophilicity $[2,11]$. While HILIC is the more convenient mode of separation than ONP, its unique retention mechanism still poses several disadvantages, such as lower reproducibility (compared to adsorption-based ANP) and a long column equilibration time; which arises from the fluctuations in surface water thickness and composition. In addition, the surface of typical silica materials in HILIC columns are rich in Si-OH groups that tend to deteriorate chromatographic peak shapes because of the unfavorable electrostatic interaction between silanols and analyte molecules [12]. Despite these drawbacks, HILIC is considered a better alternative than ONP and IPC for the analysis of polar compounds.

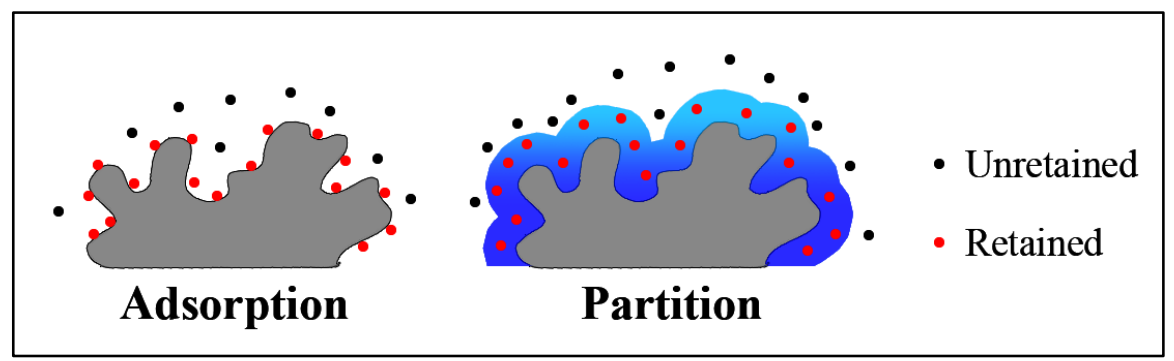

Figure 5. Scheme of Adsorption and Partition-based Retention Mechanism. 


\subsubsection{Aqueous Normal Phase (ANP)}

Aqueous normal phase (ANP) is a relatively new separation mode introduced by Pesek et al. (2005) [13]. This mode is only achievable through special stationary phases developed with the TYPE-CTM silica hydride materials (See Section 1.4.2 for details) $[13,14]$. As the term suggests, the mobile phase composition in ANP consists of water and miscible organic solvents such as acetonitrile and methanol. In ANP stationary phases, about $95 \%$ of surface Si-OH groups have been replaced with silicon-hydride group $[14,15]$. In another words, ANP lacks a feature to form immobilized water layer to employ partition mechanism. Thus, ANP retention of polar molecules is facilitated by analyte adsorption similarly to ONP (Figure 6). Overall, some of the drawbacks observed in the earlier approaches (as discussed in Section 1.3.1-1.3.3) have been reduced or no longer present in the ANP mode.

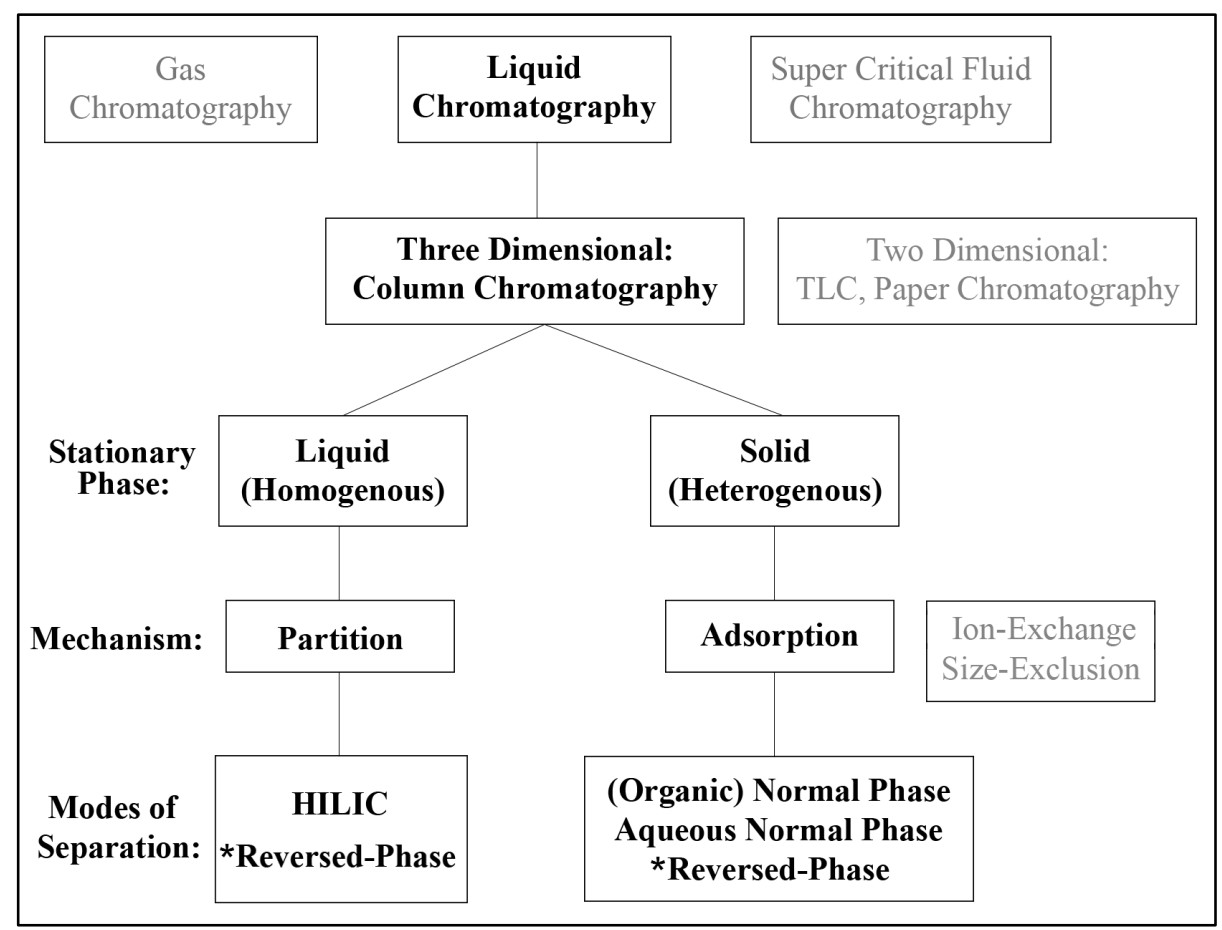

Figure 6. Classification of Chromatography. 


\subsection{Column Packing Materials}

\subsubsection{Evolution of Silica Materials}

Some of the general requirements for column packing materials include high mechanical durability, chemical stability, and so on. In modern liquid chromatography, silica is the most commonly used solid support among others (e.g., polymer and metal oxide), because silica is more cost-efficient, has chemically-adjustable surface, and it can be customized to the desired surface area, particle diameter, and pore size.

The earliest form of silica particles was large, irregular-shaped, and widely distributed in pore sizes $[6,12]$. Consequently, chromatographic results were often irreproducible. Additionally, irregular-shaped particles disintegrate and generate fine particles over time under high-pressure stress, resulting in shorter column life and/or clogging of the chromatographic system.

The second-generation type-A silica (not to be confused with silica gel classification) has a uniform spherical shape, which significantly improved chromatographic reproducibility and stability as a column. One limitation of type-A material is the presence of trace metal impurities (e.g., Al, Fe, Ni) [16] in the silica crystal lattice; which will increase the acidity of silanols and cause peak tailing when analyzing bases or chelator compounds $[17,18]$. This issue has led to the development of type-B silica, a low-metal content high purity material developed using an acid-washing process [16]. Today, type-B silica is used in the majority of HPLC columns; yet, there is significant room for improvement. For instance, surface silanol groups are still present and contribute to unwanted interactions with basic analyte solutes. To overcome this 
drawback, a process called end-capping is commonly used to react the surface silanols with a small silane reagent such as trimethylchlorosilane (TMCS; $\left.\left(\mathrm{CH}_{3}\right)_{3} \mathrm{SiCl}\right)$. Due to steric hinderance, end-capping does not completely react with every accessible silanol on the surface. These bonded silanes could somewhat act as a barrier between analyte solutes and unreacted silanols; therefore, the unwanted electrostatic interactions can be reduced. Unfortunately, the reaction leaves about $50 \%$ of silanols unreacted even if the smallest silane group was used; moreover, the bonded end-capping group becomes hydrolyzed at any $\mathrm{pH}<3$ [16]. Thus, the operational $\mathrm{pH}$ range of typical end-capped columns are limited to about $\mathrm{pH} 5-8$, where $\mathrm{pH}$ is not excessively high to cause the dissolution of the silica support itself [12].

\subsubsection{TYPE-C ${ }^{\text {TM }}$ Silica Hydride}

Silica hydride material, or TYPE-C ${ }^{\text {TM }}$ silica, was developed in the late 80 's by Dr. Joseph Pesek and Dr. Junior Sandoval [19] as a potential solution to the disadvantages exhibited by ordinary silica materials (Figure 7).

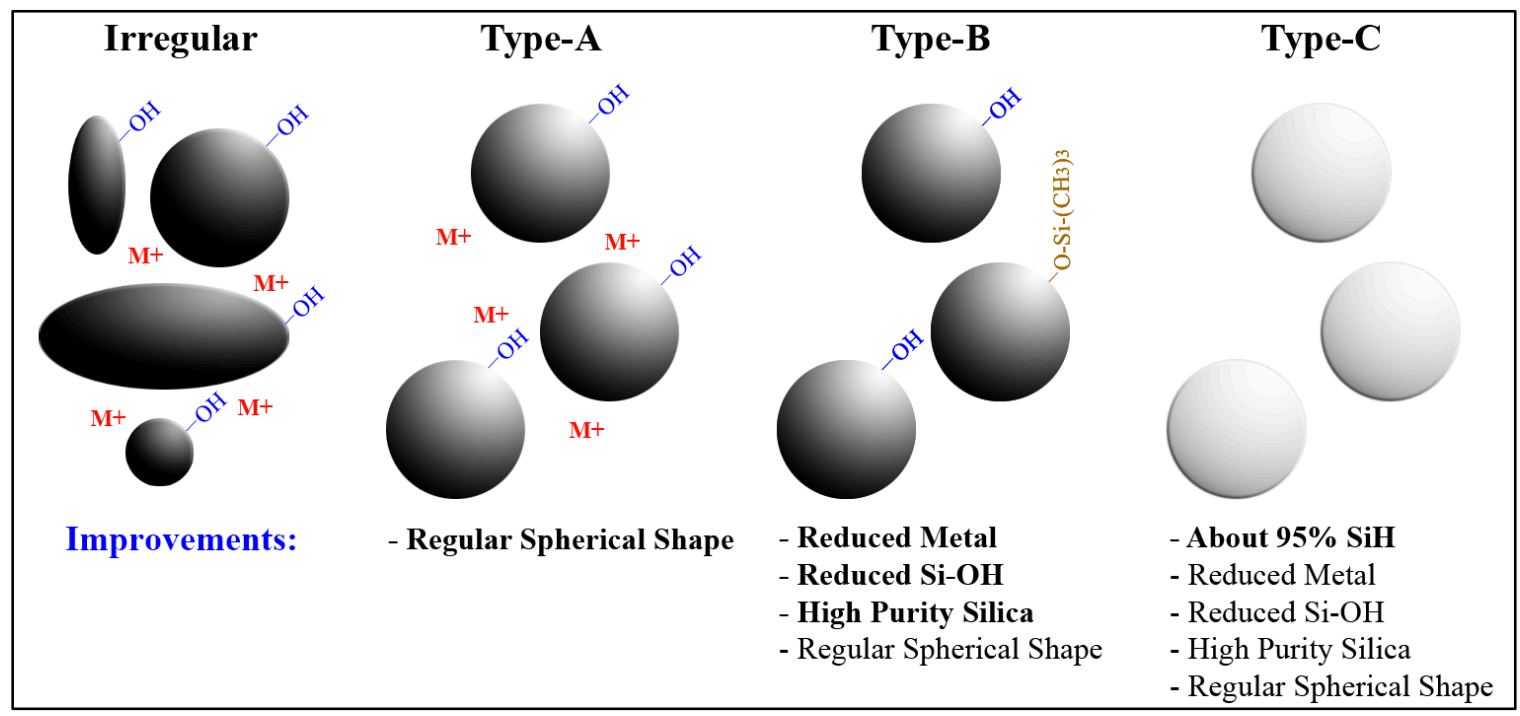

Figure 7. Evolution of Chromatographic Silica Materials. 
A study with $\mathrm{Si}^{29} \mathrm{CP}-\mathrm{MAS} \mathrm{NMR}$ and elemental hydrogen analysis has determined the surface coverage by $\mathrm{Si}-\mathrm{H}$ groups to be about $95 \%$ after the treatment with the silanization process $[14,15]$.

One way of synthesizing such hydride materials is by an acid-catalyzed condensation reaction between the silanols and triethoxysilane (TES; $\left.(\mathrm{OEt})_{3} \mathrm{SiH}\right)$ reagent (Figure 8). This one-step process requires only a small amount of acid catalyst and can be carried out in a non-dry environment. HPLC columns made with silica hydride materials are currently licensed and sold by MicroSolv Technologies, Inc. (Leland, NC) under the Cogent ${ }^{\mathrm{TM}}$ brand name.

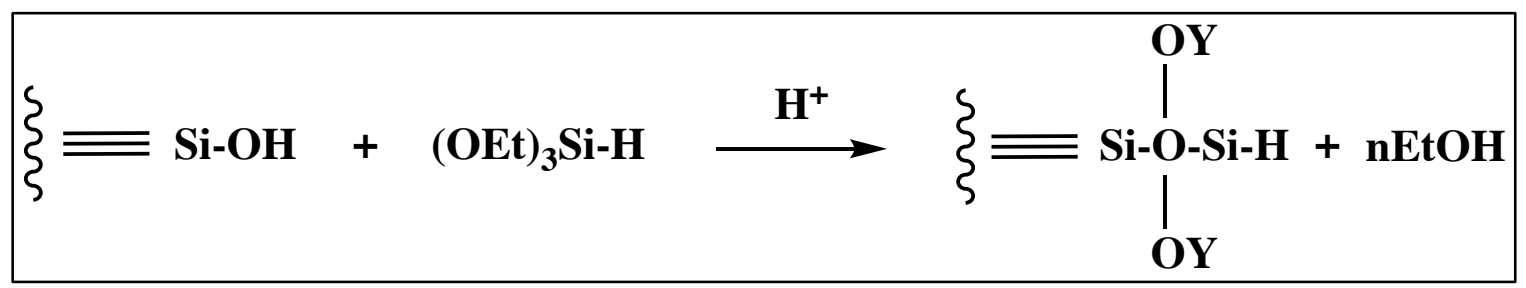

Figure 8. Silanization for Si-H Formation.

\subsubsection{Surface Modification of Silica}

Traditionally, silica surface is modified by etherification of $\mathrm{Si}-\mathrm{OH}$ group with organic alcohol (R-OH) to yield a Si-O-C linkage. However, this silyl ether linkage is known to be hydrolytically unstable in aqueous conditions [20-23]. A second approach is organosilanization, which is one of the most commonly used methods for the development of HPLC columns [22]. In this reaction, a mono- or tri-functionalized organosilane reagent $\left(\mathrm{X}-\mathrm{SiR}_{2}{ }_{2} \mathrm{R}, \mathrm{X}_{3}-\mathrm{SiR} ; \mathrm{X}=-\mathrm{Cl}\right.$, -OMe, -OEt) can be used to form a SiO-Si-C bond; reaction at a single reactive site produces a "monomeric" phase, and three groups provide "polymeric" phases. The polymeric phases exhibit higher hydrolytic 
stability; however, it is known to have lower reproducibility than the monomeric phases [20].

The third approach involves a two-step synthesis starting with chlorination of the silica surface followed by alkylation with a Grignard reagent or an organolithium compound. A direct $\mathrm{Si}-\mathrm{C}$ bond is known to be more hydrolytically stable than a conventional Si-O-C bond. Yet, this approach has been a less popular choice due to moisture-sensitive nature of the synthetic procedures [20,22].

\subsubsection{Hydrosilylation}

Hydrosilylation (or "hydrosilation") is an alternative one-step reaction process to form a hydrolytically stable Si-C bond (Figure 9). Although, this approach is limited only to the silica materials previously treated with silanization process (see Section 1.4.2 for details).

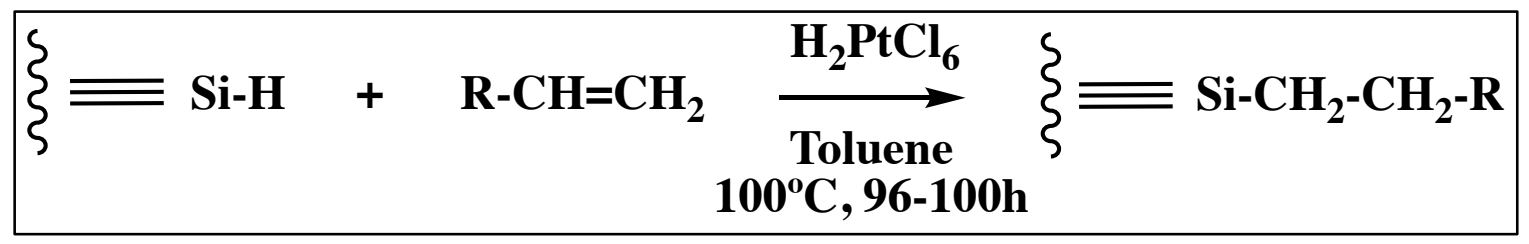

Figure 9. Hydrosilylation for the Production of Bonded Stationary Phase.

Many forms of catalysts can be utilized to perform this reaction, such as metal complexes and free-radical initiators. Of all catalysts, platinum has been one of the most popular choice after the discovery of hexachloroplatinic (IV) acid (Speier's catalyst; $\mathrm{H}_{2} \mathrm{PtCl}_{6} \cdot 2$-propanol) in the late 1950 's, which has remarkably high catalytic activity compared to other late transition metals (LTM) $[24,25]$. The mechanism of LTMcatalyzed hydrosilylation of alkenes was proposed by Chalk and Harrod (Figure 10) [26, 27]. 


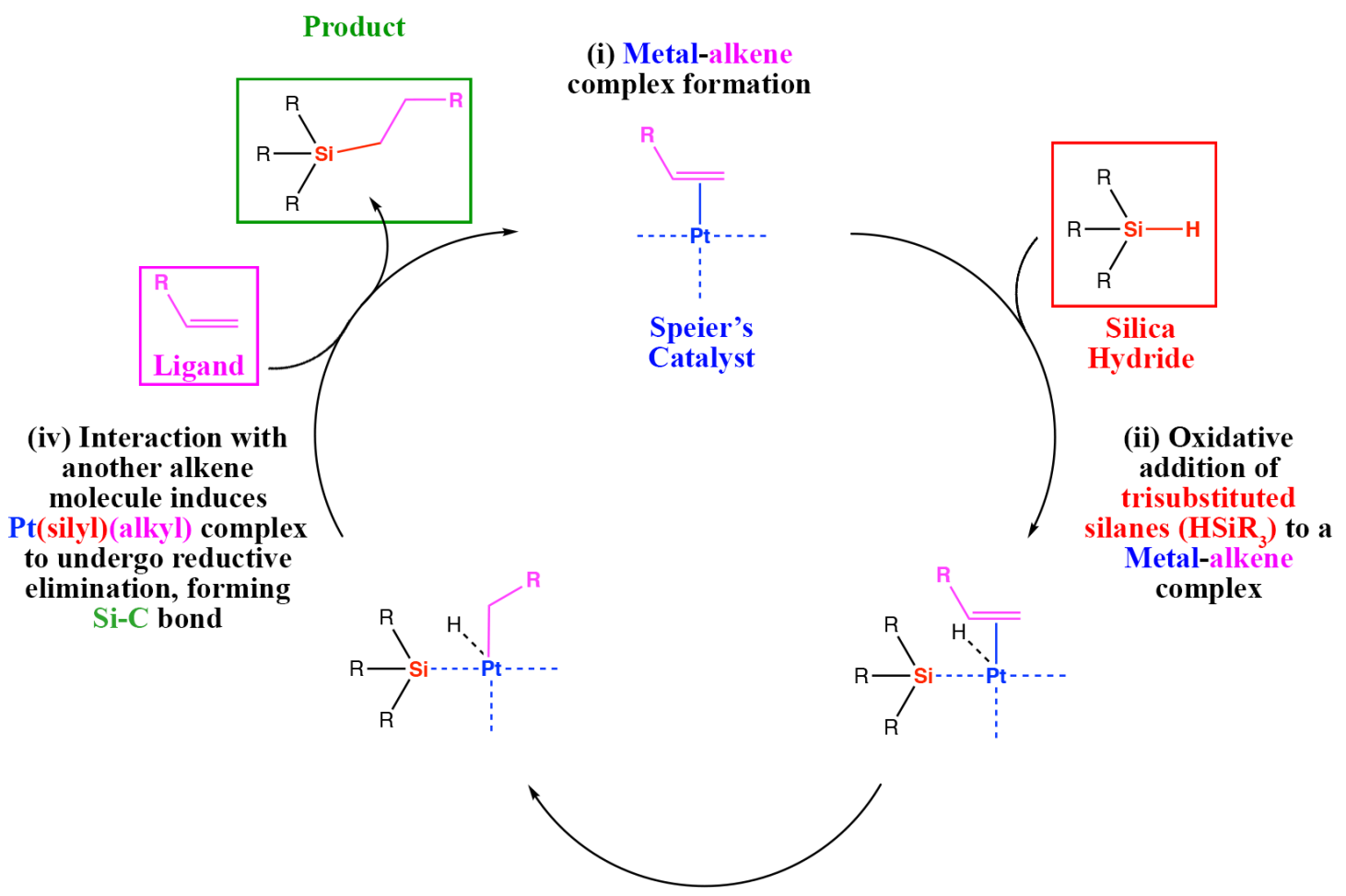

(iii) Migratory

insertion of

alkene into the

Pt-H bond

Figure 10. The Chalk-Harrod Mechanism of Olefin Hydrosilylation.

The catalytic cycle is initiated with activation of the Pt(IV) metal by a formation of the metal-alkene complex, also known as the "induction period" (i) [28-30], followed by the oxidative addition of hydrosilane to the metal-alkene complex that generates a metalhydrido-silyl intermediate (ii). Migratory insertion of the metal-bound alkene to metalhydrogen bond, also known as hydrometallation forms a metal-silyl-alkyl complex (iii). Finally, interaction with another alkene molecule induces reductive elimination of the $\operatorname{Pt}($ silyl)(alkyl) complex and leads to a formation of Si-C bond (iv).

Commonly, the reaction is used for the addition of ligands with a terminal alkene group across the $\mathrm{R}_{3} \mathrm{Si}-\mathrm{H}$ bond to form an $\mathrm{R}_{3} \mathrm{Si}-\mathrm{C}-\mathrm{C}-\mathrm{R}$ '. Some studies suggest it is also 
possible to attach olefins with non-terminal alkenes, alkynes, or even a cyano group [3133]. This unique feature opens new possibilities for developing stationary phases that were difficult to achieve with conventional synthetic approaches.

\subsection{Characteristics of TYPE-CTM Silica Columns}

\subsubsection{Surface Properties of Silica Hydride}

Since the TYPE-CTM silica surface is largely populated with nonpolar Si-H groups, there is no unintended retention of basic analytes via electrostatic attraction by $\mathrm{Si}-\mathrm{OH}$ group. Instead, bare silica hydride possesses more RP-like characteristics in comparison to silanol-rich ordinary silica materials (Figure 11).

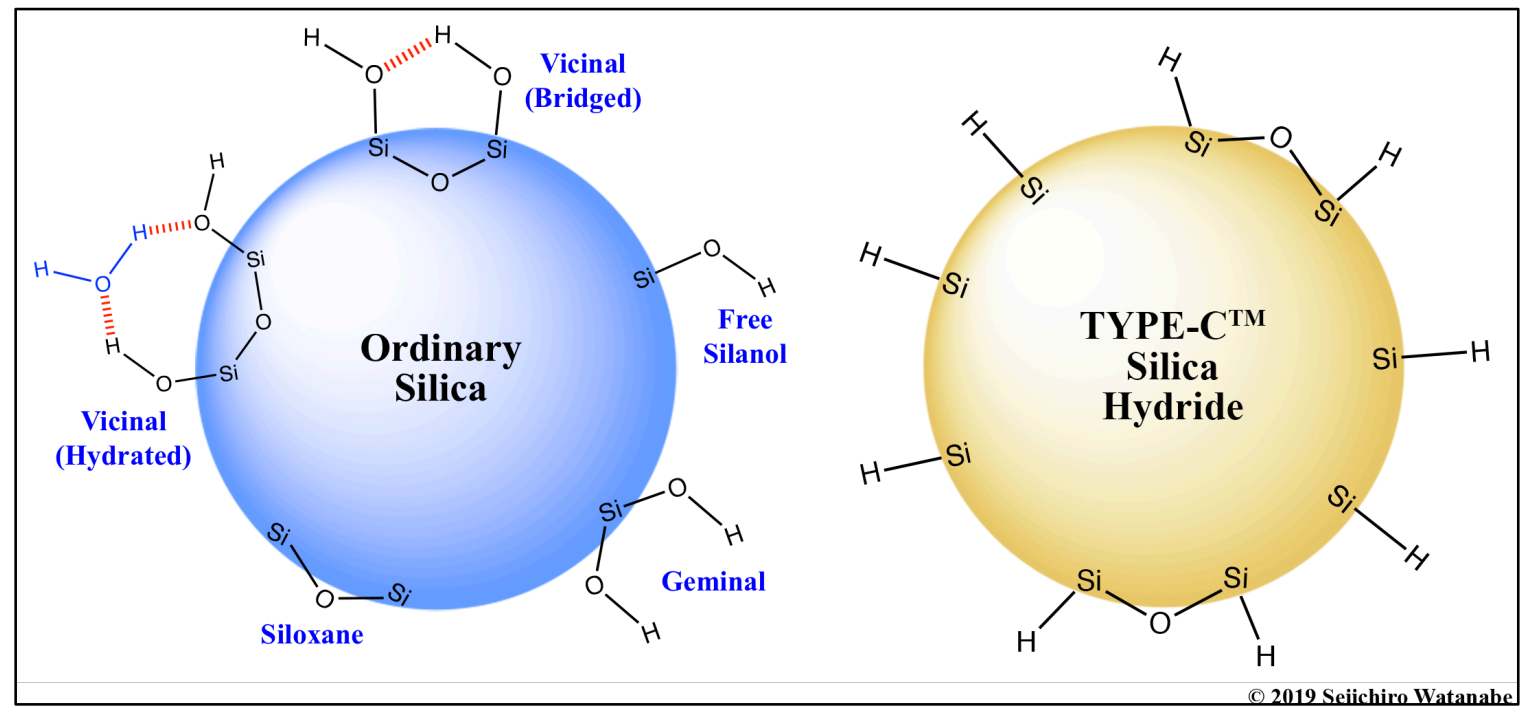

Figure 11. Comparison of the Two Silica Surfaces.

One way to demonstrate chromatographic differences between the two materials is by measuring the retention behavior of uracil, pyridine, and phenol under RP conditions. Uracil is used as a control for an unretained component (a void marker); pyridine is a basic analyte which could be retained by silanol-rich columns, and phenol is a typical example of a hydrophobic compound easily retained by RP columns. The bare ordinary 
silica column does not retain uracil and phenol very well; however, pyridine is retained due to its interaction with the surface silanols. In contrast, silica hydride columns exhibit significant increase in phenol retention while pyridine is only slightly more retained than uracil $[13,14]$. In case of non-end-capped RP column, pyridine could be retained longer than phenol due to its significant silanol activity (Figure 12).

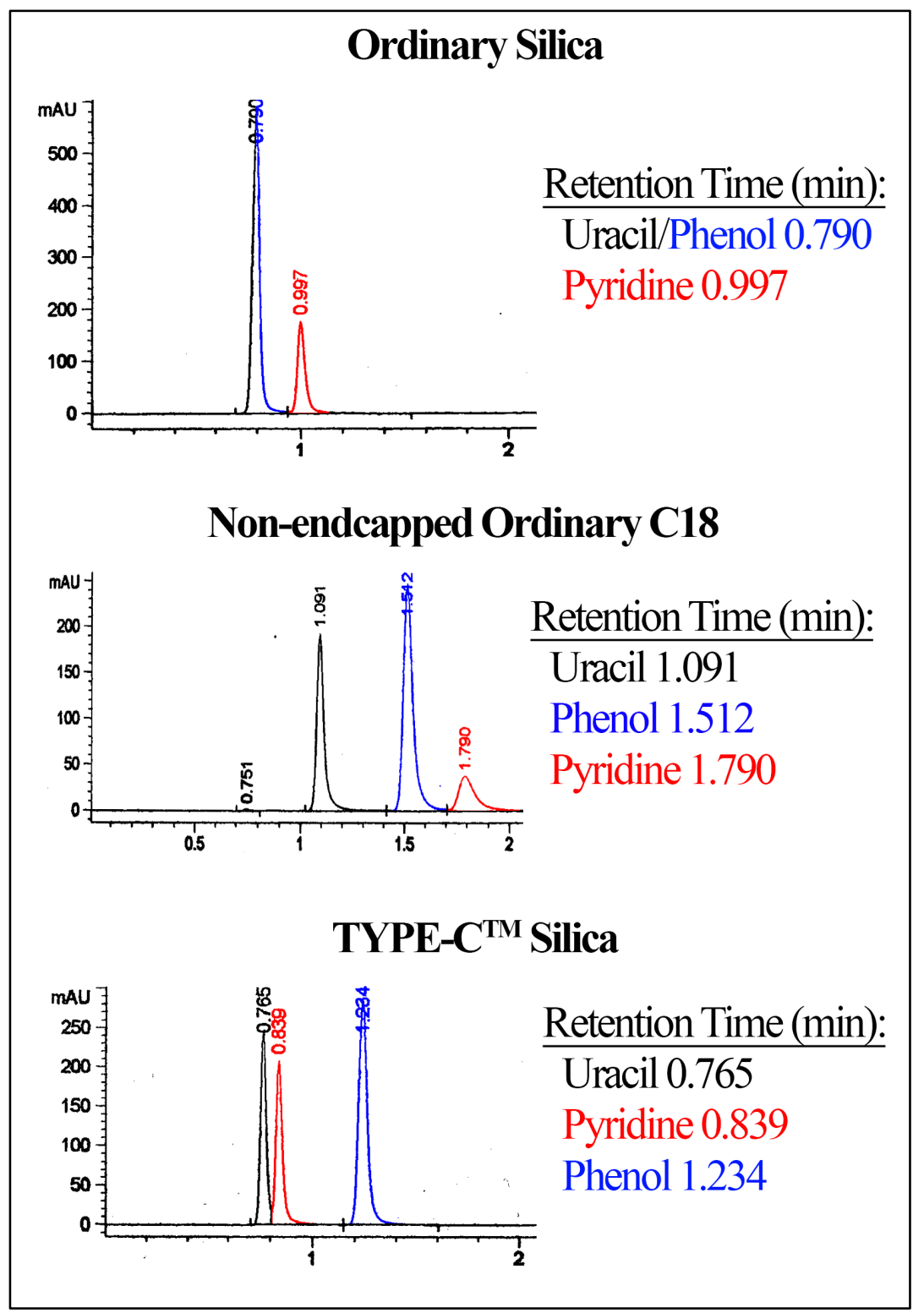

Figure 12. Chromatographic Property of Silica Hydride-based Stationary Phase. (Adapted from Pesek et al., 2005 [13]) 
The lack of silanol groups and their replacement with silica hydride poses numerous additional positive influences on chromatographic performance. For instance, the silica hydride surface does not accumulate strongly adsorbed water layers $(<0.5$ monolayer $)$ as much as the silanol-rich ordinary silica surface does (3-9.5 monolayers) (Figure 13).

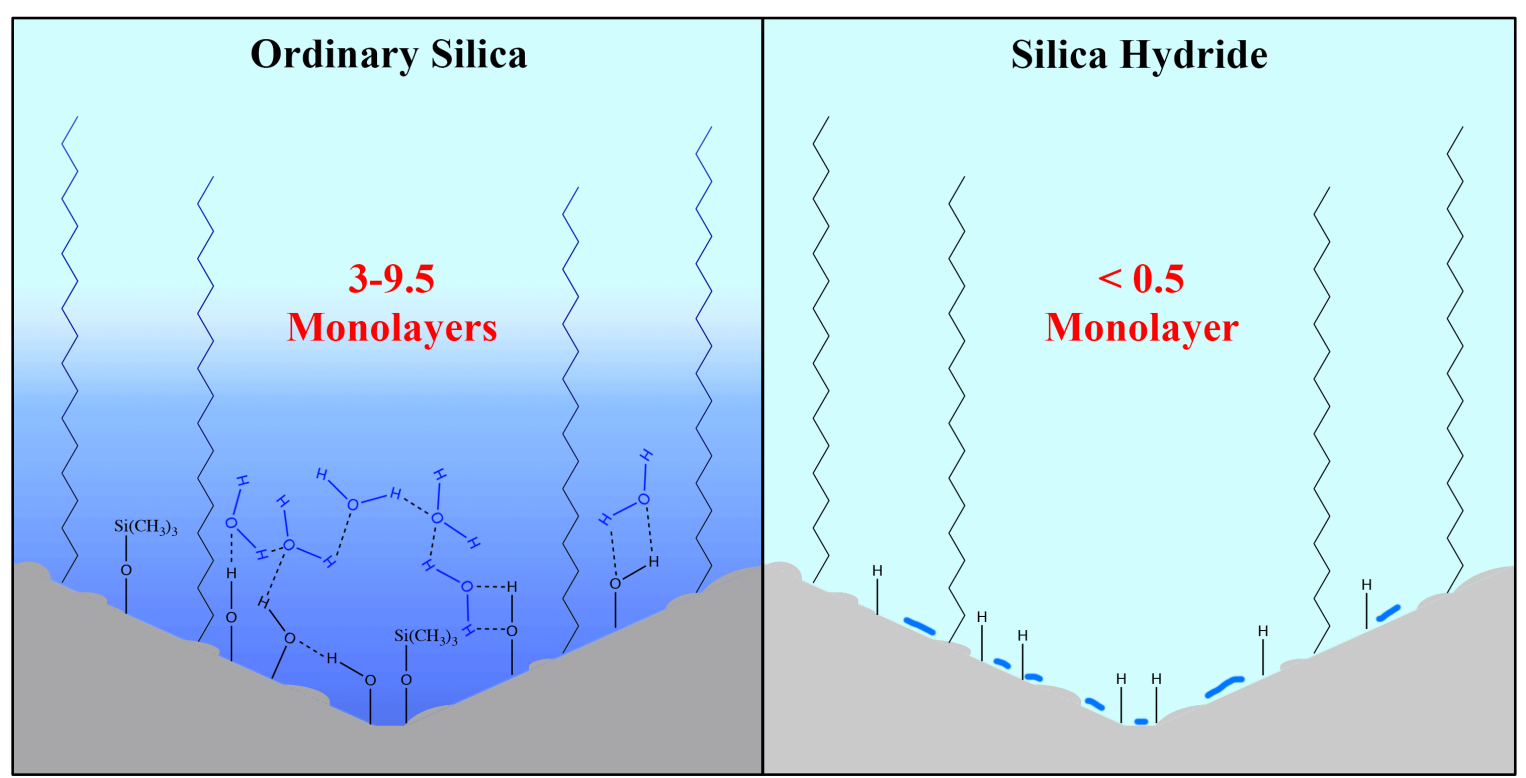

Figure 13. Amount of the Adsorbed Water Monolayer(s) by Two Silica Materials.

Partitioning that takes place on the surface can vary due to the thickness of water layer, which also depends on mobile phase composition. Since the silica hydride surface is much more stable in this sense, it is known to produce greater reproducibility and precision for polar molecule analysis, and shorter column equilibration time compared to ordinary silica columns [34].

\subsubsection{Polar Analyte Retention by ANP Chromatography}

Another unique property of the silica hydride material is its ability to operate in the normal phase mode (both ANP and ONP) in addition to the RP mode. As discussed in Section 1.3.3-1.3.4, silica hydride materials do not form strongly adsorbed water layers 
like the ordinary silica and HILIC materials do. Thus, polar analyte retention mechanism is distinct from the HILIC mode's partition mechanism but rather comparable to ONP's adsorption-based mechanism. This is supported by different trends in retention exhibited by the two modes in changing mobile phase additive concentration. While there are some exceptions, the HILIC mode is generally known to increase polar analyte retention as the additive (e.g., formic acid, ammonium acetate) concentration increases. This is due to higher salt content increases the thickness of hydration layer, thereby extending the polar analyte retention [35]. In contrast, ANP retention of polar analytes decreases as the additive concentration increases [36].

Polar analyte retention by silica hydride seems to be counter-intuitive considering its surface being nonpolar.

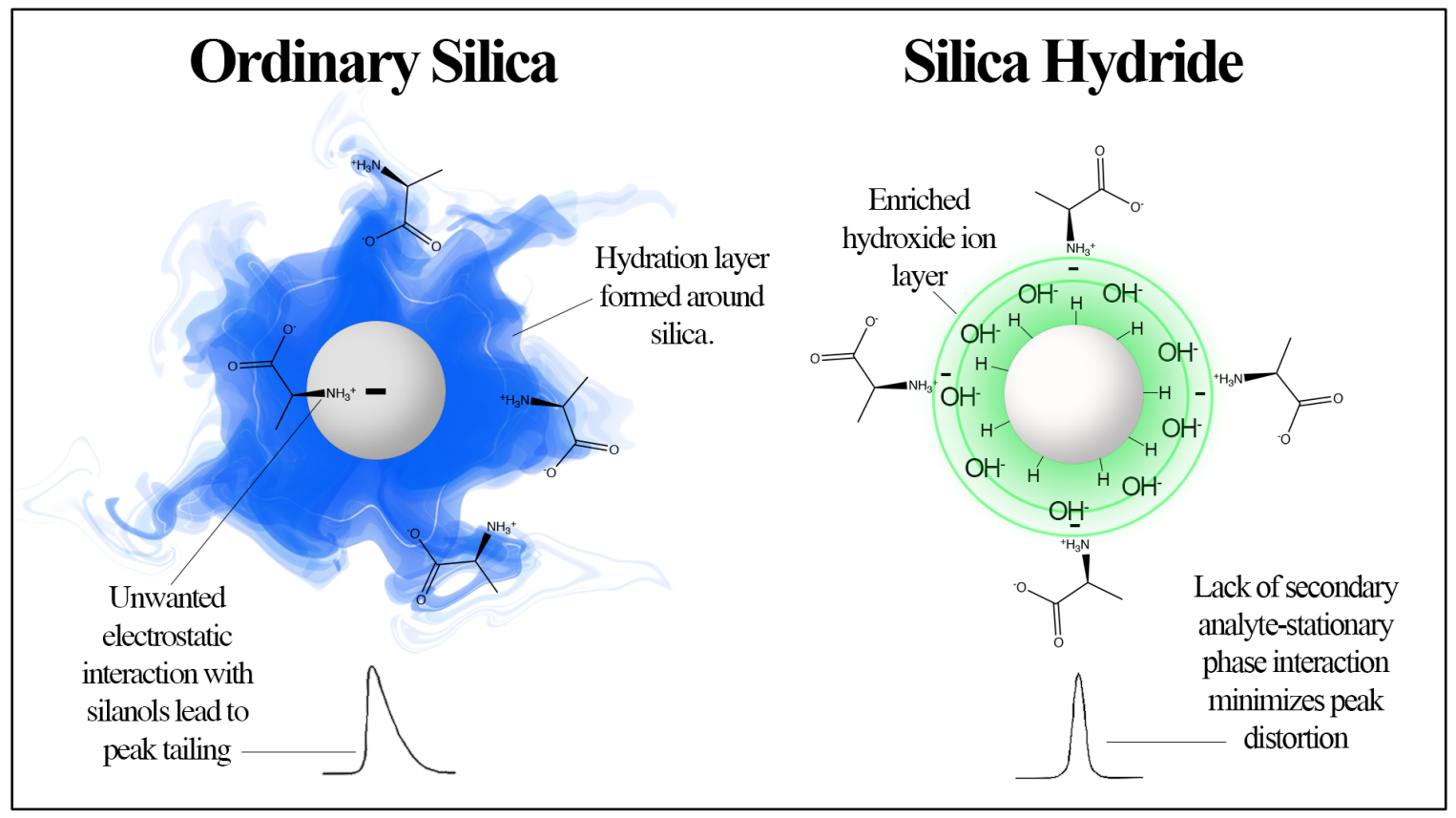

Figure 14. Surface Property Differences Among the Two Silica Materials. 
To determine the mechanism behind this behavior, Kulsing et al., (2014) conducted zeta potential measurements on hydride particles in aqueous-organic media. According to their study, the surface of silica hydride is negatively charged due to the accumulation of hydroxide/negative ions, which can be explained by the collective dipole-moment fluctuation suppression theory (Figure 14) $[37,38]$. This phenomenon is often associated with the "oil droplet in water" analogy, which also behaves like a negatively charged ion upon the formation of micelles and attracts cations to its surface [39].

\subsubsection{Dual Retention Capabilities}

The ability of hydride-based columns to operate in both normal and reversed phase modes is often called a "dual retention" property. This feature can be observed on the same column in two separate runs [12], or simultaneously in a single run [40]. In Figure 15 , the hydrophobic glyburide (hydrophobicity coefficient $-\log \mathrm{P}=4.79$ ) is retained longer than hydrophilic metformin ( $\log \mathrm{P}=-2.64)$ on a $\mathrm{C} 18 \mathrm{RP}$ column at $50 \%$ organic composition. Under these conditions, RP is very likely the dominant mode of retention. However, as the organic composition increases to $85 \%$, the retention of metformin becomes greater than glyburide; thus, ANP becomes dominant over the RP mode. 


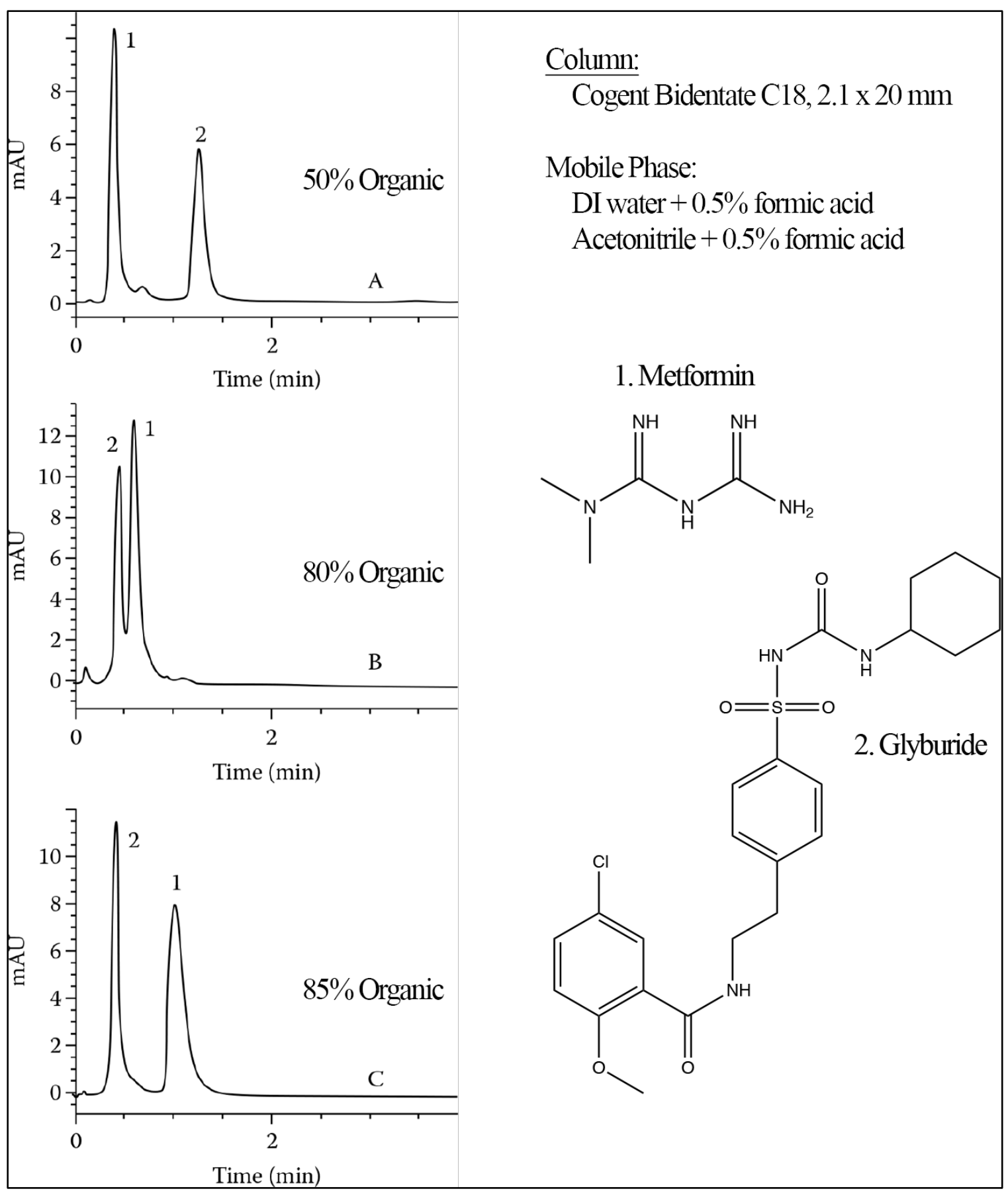

Figure 15. Retention of Hydrophilic and Hydrophobic Analytes by a Single Column. (Adapted from Pesek et al., 2005 [13])

When the retention time of polar/nonpolar molecules is plotted as a function of $\%$ organic, it gives a characteristic "U-shaped" profile [36], (Figure 16). 


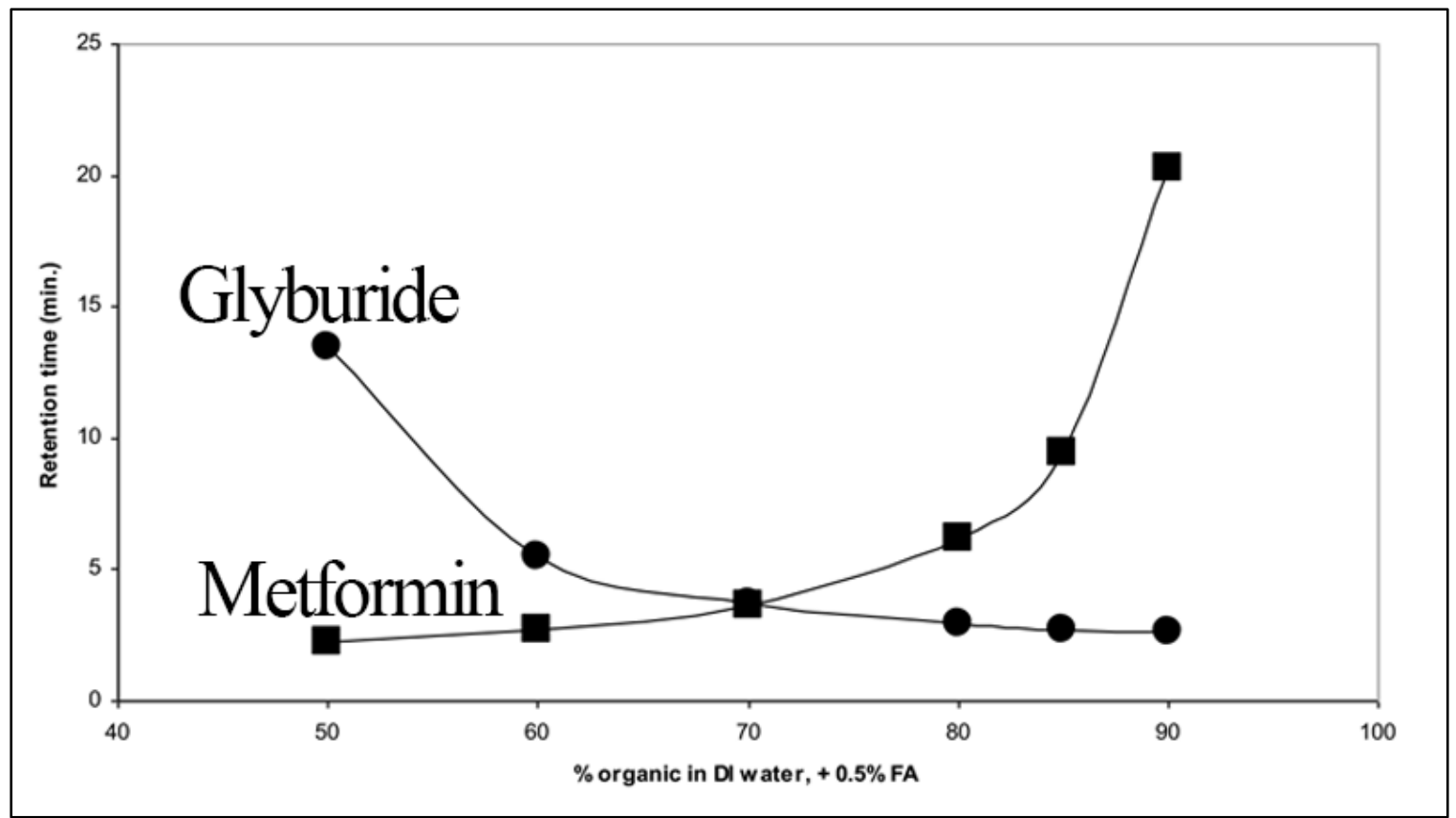

Figure 16. The Analyte Retention Profile of a Silica Hydride-based C18 Column. (Adapted from Pesek et al., 2005 [13])

The dual retention feature is particularly useful when analyzing molecules with amphipathic properties, or a mixture containing analytes with ranges of hydrophobicity. Boysen et al., (2011) have demonstrated the simultaneous separation of both hydrophilic and hydrophobic peptides, with hydrophobicity coefficients ranging from $\log \mathrm{P}=-2.31$ to $\log \mathrm{P}=11.34$, in a single ANP gradient run [40]. Figure 17 shows the retention of five relatively hydrophilic peptides for more than 15 mins. The two relatively non-polar peptides with close hydrophobicity parameters were eluted at much earlier time-point, but the peaks are still almost fully resolved. In summary, a single hydride-based stationary phase allows for the retention and selectivity for a much larger variety of molecules. 


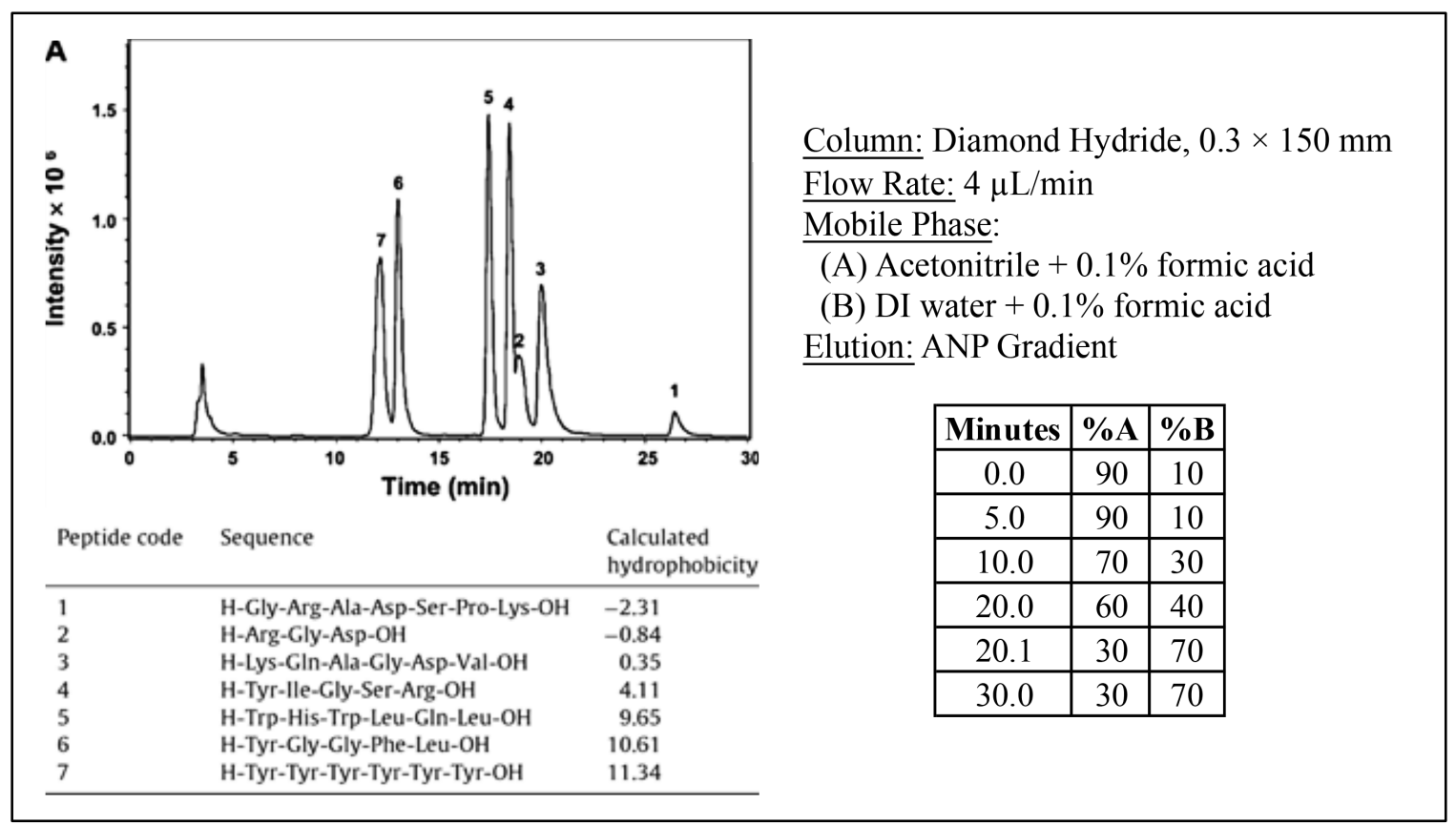

Figure 17. Retention of Hydrophilic and Hydrophobic Peptides in a Single Run. (Adapted from Boysen et al., 2011 [40])

\subsection{Research Goals}

The general objectives of this study are three-fold: (1) to develop and characterize novel silica hydride-based stationary phases; (2) to evaluate the chromatographic performance of new column materials; and (3) to discover new bioanalytical applications of TYPE-C ${ }^{\text {TM }}$ silica hydride stationary phases and ANP chromatography.

In this study, four new HPLC columns were developed from TYPE-C ${ }^{\text {TM }}$ silica hydride provided by MicroSolv Technologies, Inc. and with four olefin materials: allyloxy-DL-alpha-tocopherol (TOCO), allylpentafluorobenzene (PFP), 1-eicosene (C20), and 10-undecenyloxycarbonyl-leucine (LEU). Upon completion of the hydride surface modification, DRIFTS, $\mathrm{Si}^{29}$ CP-MAS solid-state NMR, and elemental carbon analysis were utilized as a confirmation tool for reaction products and to predict the chromatographic properties of the materials. Another aim in this project is to better 
understand the physical properties of silica hydride materials that allows for the materials to operate in the ANP mode. Thus, silica hydride nano-particle behavior in aqueous media was investigated using MMFF94 molecular dynamics (MD) simulations, and variable-temperature DRIFTS (VT-DRIFTS) was used to study the desorption of water from the silica lattice.

The second part of the study evaluates the general chromatographic properties of the new columns based on the following criteria: dual retention property, hydrophobicity, metal-ion activity, silanophilicity, and selectivity. The analyte selectivity is investigated by screening several compound types including amino acids, steroids, nucleotides, neurotransmitters, sugars, and nootropics. Understanding the analyte-column interaction is a critical step in choosing the right column for a project and successful method development.

The final step is to discover new applications of hydride-based stationary phases and ANP chromatography in various bioanalytical settings by using the newly developed columns. Fields of interest are pharmaceutical, biological sample analysis, food science, metabolomics, and structural proteomics.

\section{EXPERIMENTAL}

\subsection{Materials}

\subsubsection{Preparation of the Bonded Phases}

The TYPE-C ${ }^{\text {TM }}$ silica hydride was obtained from MicroSolv Technology, Inc.

(Leland, NC). The hydride material has an average particle size of $4 \mu \mathrm{m}, 100 \AA$ pore size, and the surface area of $400 \mathrm{~m}^{2} / \mathrm{g}$. Three olefins purchased from Gelest, Inc. (Morrisville, 
PA) were allyloxy-DL- $\alpha$-tocopherol (TOCO), allylpentafluorobenzene (PFP), and 1eicosene (C20). The fourth material, 10-undecenyloxycarbonyl-leucine (LEU), was obtained from Professor Shamsi at the Department of Chemistry at Georgia State University. Hydrosilylation was carried out in an anhydrous environment using toluene as the primary reaction solvent. The Speier's catalyst was prepared by dissolving hexachloroplatinic (IV) acid in 2-propanol. Other reagents and equipment used for the synthesis can be found in Table 1 and Table 2. Synthetic procedures are further discussed in Section 2.3.2.

Table 1. Reagents Used for Hydrosilylation.

\begin{tabular}{|c|c|c|}
\hline Name & Vendor & Catalog \# \\
\hline Silica Hydride & $\begin{array}{c}\text { MicroSolv } \\
\text { Technologies, Inc. }\end{array}$ & - \\
\hline Dichloromethane & EM Science & DX0838-1 \\
\hline Toluene & Sigma Aldrich & $24451-1$ \\
\hline Ethyl Ether & Fisher Scientific & E138-1 \\
\hline 2-Propanol & Fisher Scientific & A451-4 \\
\hline $\begin{array}{c}\text { Hexachloroplatinic (IV) } \\
\text { acid }\end{array}$ & Riedel-de Haen & 12506 \\
\hline Potassium Bromide & Harshaw/Filtrol & $9-5-0002$ S \\
\hline $\begin{array}{c}\text { KBr Powder Packets } \\
\text { Calcium Chloride } \\
\text { (anhydrous) }\end{array}$ & $\begin{array}{c}\text { Thermo Fisher } \\
\text { Scientific } \\
\text { Chemical Ltd }\end{array}$ & $0016-032$ \\
\hline Allylpentafluorobenzene & Gelest, Inc. & ENEA0378 \\
\hline $\begin{array}{c}\text { Allyloxy-DL- } \alpha- \\
\text { tocopherol }\end{array}$ & Gelest, Inc. & ENEA0374 \\
\hline 1-Eicosene, 90\% & Gelest, Inc. & ENEE1020 \\
\hline $\begin{array}{c}10- \\
\text { undecenyloxycarbonyl- } \\
\text { leucine }\end{array}$ & $\begin{array}{c}\text { Georgia State } \\
\text { University }\end{array}$ \\
\hline
\end{tabular}


Table 2. Miscellaneous Equipment Used During Synthesis.

\begin{tabular}{|l|c|c|}
\hline \multicolumn{1}{|c|}{ Name } & Vendor & Catalog \# \\
\hline $\begin{array}{l}\text { 19/22 250 mL 3-necked round- } \\
\text { bottom flask }\end{array}$ & Kimax USA & - \\
\hline \multirow{2}{*}{ 19/38 West Condenser } & Pyrex & - \\
\cline { 2 - 3 } & Kimax USA & - \\
\hline 1L Filtering Flask & Pyrex & 5340 \\
\hline $\begin{array}{l}\text { 250 mL Flexible Fabric Heating } \\
\text { Mantle }\end{array}$ & Glas-col Apparatus Company & O402 \\
\hline $\begin{array}{l}\text { 90M Sintered Glass Funnel (600 } \\
\text { mL) }\end{array}$ & Kimax USA & - \\
\hline Analytical Balance & Mettler Instrument Corp. & AE200 \\
\hline Drying tube 19/22 & ACE GLASS INC. & - \\
\hline Dyla-Dual Hot Plate/Stirrer & VWR Scientific & $58849-001$ \\
\hline $\begin{array}{l}\text { Enlarging Adaptor (24/40 -> } \\
\text { 19/22) }\end{array}$ & Kimax USA & - \\
\hline $\begin{array}{l}\text { Thelco Model 17 Laboratory } \\
\text { Oven }\end{array}$ & PCE GLASS INC. & - \\
\hline Thermometer (-20-110C) & Labcraft & 31478 \\
\hline $\begin{array}{l}\text { Total Immersion Precision } \\
\text { Thermometer }\end{array}$ & VWR Scientific & $61016-026$ \\
\hline Vacuum Oven & VWR Scientific & VWR 1410 \\
\hline \multirow{2}{*}{\begin{tabular}{l} 
Variable Transformer \\
\cline { 2 - 3 }
\end{tabular}} & Scientific Products & $122-0003$ \\
\hline
\end{tabular}

\subsubsection{Column Performance Evaluation}

Reagents used for mobile phase and sample preparation in all HPLC/LC-MS experiments are listed in Table 3. The list of analytes used for the characterization of basic column performance and miscellaneous materials are shown in Table 4 and Table 5, respectively. 
Table 3. Reagents Used for Mobile Phase Preparation.

\begin{tabular}{|l|c|c|}
\hline \multicolumn{1}{|c|}{ Name } & Vendor & Catalog \# \\
\hline Milli-Q Water & $\begin{array}{c}\text { Millipore Water Purification } \\
\text { System }\end{array}$ & - \\
\hline Methanol & GFS Chemicals & 1679 \\
\hline Methanol & Fisher Chemical & A452-4 \\
\hline Acetonitrile & EMD & AX0142-1 \\
\hline Formic Acid & Sigma Aldrich & F-0507 \\
\hline Dimethyl Sulfoxide & EM Science & MX1458-1 \\
\hline Potassium Phosphate, Dibasic & J.T. Baker & 493810 \\
\hline Potassium Phosphate, Monobasic & EM Science & PX1565-1 \\
\hline Ammonium Hydroxide & J.T. Baker & 2856564 \\
\hline
\end{tabular}

Table 4. Compounds Analyzed for Column Performance Evaluation.

\begin{tabular}{|l|c|c|}
\hline \multicolumn{1}{|c|}{ Name } & Vendor & Catalog \# \\
\hline SRM 870 & NIST & - \\
\hline Uracil & Sigma Aldrich & - \\
\hline Naphthalene & J. T. Baker Chemical Co. & - \\
\hline Acenaphthene & Packard & - \\
\hline Anisole & N/A & - \\
\hline Ortho-dimethoxybenzene & Janssen Chimica & SJSU Barcode: C010326 \\
\hline Meta-dimethoxybenzene & Sigma Aldrich & SJSU Barcode: C006135 \\
\hline Para-dimethoxybenzene & EKC & SJSU Barcode: C006137 \\
\hline Adenine & - & - \\
\hline Adenosine & - & - \\
\hline Caffeine & - & - \\
\hline Glyburide & - & - \\
\hline Potassium Nitrate & - & - \\
\hline
\end{tabular}


Table 5. Miscellaneous Materials Used in the Study.

\begin{tabular}{|l|c|c|}
\hline \multicolumn{1}{|c|}{ Equipment } & Vendor & Catalog \# \\
\hline $0.45 \mu \mathrm{m}$ Nylon Membrane Filter & MicroSolv Technologies, Inc. & 58545-N47 \\
\hline Syringes, $3 \mathrm{~mL}$ & MicroSolv Technologies, Inc. & 58903-S-C \\
\hline Clinical Centrifuge & International Equipment Co. & Model CL \\
\hline Bond Elut Certify II SPE & Varian & $1211-3051$ \\
\hline UCT Clean XCEL I & United Chemical Technologies & CSXCE103 \\
\hline UCT Clean XCEL II & United Chemical Technologies & CSXCE2103 \\
\hline MagNA Lyser Green Beads & Roche & 03 358 941 001 \\
\hline HPLC vials, Screw Top 2 mL & MicroSolv & $9502 S-0 C V$ \\
\hline AQTM Brand RSA vials & MicroSolv & 9509 S-1WAP-RS \\
\hline Bransonic 12 Ultrasonic Cleaner & Branson & - \\
\hline Reacti-Therm ${ }^{\text {TM Heating Module }}$ & Pierce & 18800 \\
\hline Deluxe Mixer Vortex & Scientific Products & S8220 \\
\hline Thermolyne Mixer & Barnstead & M16715 \\
\hline Education Line - pH Meter & Mettler Toledo AG & EL20 \\
\hline Wig-L-Bug Amalgamator & Crescent & SC-40 \\
\hline
\end{tabular}

\subsubsection{Demonstration Studies}

\subsubsection{Detection of PCBNs in Formulation and Cannabis Plant Extracts}

The novel stationary phases were evaluated for analysis of phytocannabinoids in the extracts of four cannabis plants and a gummy pharmaceutical formulation of cannabidiol (CBD). A list of reference standards and samples are provided in Table 6.

Table 6. Samples and Reference Standards Used for Cannabinoids Analysis.

\begin{tabular}{|l|c|c|}
\hline \multicolumn{1}{|c|}{ Name } & Vendor & Catalog \# \\
\hline Cannabidiol (CBD) & \multirow{2}{*}{ Sigma Aldrich } & C6395 \\
d3-Cannabidiol (d3-CBD) & & C-084 \\
\hline White Fire OG (Cannabis sp. hybrid) & - & - \\
\hline Bio (Cannabis sp. hybrid) & - & - \\
\hline Super Silver Haze (Cannabis sativa) & - & - \\
\hline Granddaddy Purple (Cannabis indica) & - & - \\
\hline Kushy Punch (CBD Gummy Edible) & Caliva & - \\
\hline
\end{tabular}




\subsubsection{Retention Profile of Controlled Substances}

Bioanalytical study application was demonstrated using a human urine control (UTAK Control Level 1) containing nineteen commonly abused controlled substances (See Section 2.3.4 for details). All samples and reference standards used in the project are shown in Table 7.

Table 7. List of Materials and Standards Used for Human Urine Analysis.

\begin{tabular}{|c|c|c|}
\hline Name & Vendor & Catalog \# \\
\hline Urine Control Level 1 & UTAK & 4308 \\
\hline Heroin & Sigma Chemical Co. & H-5144 \\
\hline LSD & \multirow{7}{*}{ Cerillant Corp } & L-001 \\
\hline MDMA & & M-013 \\
\hline 4-Fluoromethcathinone & & - \\
\hline Mephedrone & & - \\
\hline N-ethylcathinone & & - \\
\hline Pentedrone & & - \\
\hline S/R-Cathinone & & - \\
\hline
\end{tabular}

\subsubsection{Metabolomics Characterization of USDA Grape Cultivars}

Metabolic profiles of five grape skin extracts were analyzed and compared using the novel silica hydride stationary phases as separation media. Reference standards and grape skin samples obtained from Dr. Gary Takeoka \& Dr. Craig Ledbetter (the United States Department of Agriculture - Agricultural Research Service - Western Regional Research Center - Healthy Processed Foods Research; USDA-ARS-WRRC-HPFR) are listed in Table 8. 
Table 8. List of Standards and Samples Used for the USDA Grape Analysis.

\begin{tabular}{|c|c|c|}
\hline Name & Vendor & Catalog \# \\
\hline Gallic Acid & \multirow{12}{*}{ Sigma Aldrich } & G7384-100G \\
\hline 3,4-Dihydroxybenzoic acid & & $37580-25 \mathrm{G}-\mathrm{F}$ \\
\hline 4-Hydroxybenzoic acid & & H20059-100G \\
\hline Syringic acid & & S6881-5G \\
\hline Caftaric acid & & $15029-5 \mathrm{MG}$ \\
\hline$(+)$-Catechin & & $\mathrm{C} 1251-5 \mathrm{G}$ \\
\hline (-)-Epicatechin & & E1753-1G \\
\hline Resveratrol & & R5010-100MG \\
\hline Procyanidin B1 & & 19542-1MG-F \\
\hline Procyanidin B2 & & 42157-1MG-F \\
\hline Quercetin 3-glucoside & & 16654-10MG \\
\hline Kaempferol 3-glucoside & & $68437-5 \mathrm{MG}$ \\
\hline Y539-194-06 & \multirow{5}{*}{$\begin{array}{l}\text { USDA-ARS- } \\
\text { WRRC-HPFR }\end{array}$} & - \\
\hline C33-30 & & - \\
\hline Scarlet Royal & & - \\
\hline Y144-169-12 & & - \\
\hline Y144-162-12 & & - \\
\hline
\end{tabular}

\subsubsection{Proton Back-exchange Reduction by ANP-coupled HDX-MS}

Silica hydride and aqueous normal phase (ANP) chromatography were evaluated for the structural proteomics assay - hydrogen-deuterium exchange mass spectrometry (HDX-MS) as a potential approach to reduce proton back-exchange during chromatographic analysis. Table 9 shows the model peptides and deuterium oxide $\left(\mathrm{D}_{2} \mathrm{O}\right)$ used in the study.

Table 9. Materials Used for ANP-HDX-MS Study.

\begin{tabular}{|l|l|c|}
\hline \multicolumn{1}{|c|}{ Name } & Vendor & Catalog \# \\
\hline$\left[{\left.\mathrm{D}-\mathrm{Ala}^{2}, \mathrm{D}-\mathrm{Met}^{5}\right] \text {-Enkephalin Acetate }}^{\mid}\right.$ & Sigma Aldrich & E-3507 \\
\hline Angiotensin II & Sigma Aldrich & A-9525-1MG \\
\hline Deuterium Oxide & Stohler Isotope Chemicals & D320 \\
\hline
\end{tabular}




\subsubsection{Selectivity Test}

Column-analyte selectivity is evaluated by testing a series of molecular species for retention and separation. The following groups of compounds were used: steroids, sugars, nucleobases, vitamins, neurotransmitters, amino acids, and nootropic drug molecules (Table 10).

Table 10. List of Analytes Tested for Column Selectivity.

\begin{tabular}{|c|c|c|}
\hline Name & Vendor & Catalog \# \\
\hline Progesterone & Sigma-Aldrich & P-0130 \\
\hline (-)-Norgestrel & Sigma-Aldrich & $\mathrm{N}-2260$ \\
\hline Estrone & Sigma-Aldrich & E-9750 \\
\hline$\Delta^{4}$-Androsten-3,17-dione & Sigma-Aldrich & A-9630 \\
\hline Adrenosterone & Sigma-Aldrich & A-6252 \\
\hline Beta-Estradiol & Sigma-Aldrich & E-8875 \\
\hline Corticosterone & Sigma-Aldrich & $\mathrm{C}-2505$ \\
\hline Prednisolone & Sigma-Aldrich & P-6004 \\
\hline $17 \alpha$-Ethynylestradiol & Sigma-Aldrich & E-4876 \\
\hline $11 \alpha$-acetoxyprogesterone & - & - \\
\hline Ethisterone & Sigma-Aldrich & $28,681-1$ \\
\hline Raffinose & Fisher Scientific Co. & - \\
\hline $\mathrm{D}(+)$-Melezitose & Sigma-Aldrich & - \\
\hline $\mathrm{D}(+)$-Maltose & JT Baker Chemical Co. & - \\
\hline D-Trehalose dehydrate & Matheson Coleman \& Bell & - \\
\hline Lactose & Pfanstiehl Laboratories Inc. & - \\
\hline Sucrose & JT Baker Chemical Co. & - \\
\hline $\mathrm{D}(+)$-Turanose & ICN Pharmaceuticals Inc. & 103167 \\
\hline Glucose & Sigma-Aldrich & - \\
\hline D-Ribose & Nutritional Biochemicals Co. & - \\
\hline $\mathrm{D}(+)-\mathrm{Xylose}$ & JT Baker Chemical Co. & - \\
\hline Adenine & JT Baker Chemical Co. & - \\
\hline Thymine & Sigma-Aldrich & - \\
\hline Guanine & - & - \\
\hline Cytosine & Nutritional Biochemicals Co. & - \\
\hline Thiamine & Matheson Coleman \& Bell & 5421 \\
\hline
\end{tabular}




\begin{tabular}{|c|c|c|}
\hline Riboflavin & - & - \\
\hline Folic Acid & Sigma-Aldrich & F7876-10G \\
\hline Pyridoxine & Matheson Coleman \& Bell & 1145 \\
\hline Biotin & KODAK & 1368448 \\
\hline (-)-Epinephrine & Sigma-Aldrich & $\mathrm{E} 4250-5 \mathrm{G}$ \\
\hline (-)-Norepinephrine & Sigma-Aldrich & A7257-1G \\
\hline Dopamine & Sigma-Aldrich & H8502-5G \\
\hline GABA & Sigma-Aldrich & A-2129 \\
\hline DL-Alanine & - & - \\
\hline L-Cysteine & - & - \\
\hline DL-Aspartic Acid & Mallinckrodt Chemical Works & - \\
\hline DL-Glutamic Acid & Sigma-Aldrich & - \\
\hline DL-Phenylalanine & JT Baker Chemical Co. & - \\
\hline Glycine & - & - \\
\hline L-Histidine & Sigma-Aldrich & - \\
\hline DL-Isoleucine & - & - \\
\hline Lysine & - & - \\
\hline DL-Leucine & - & - \\
\hline DL-Methionine & - & - \\
\hline DL-Asparagine & - & - \\
\hline DL-Proline & - & - \\
\hline L-Glutamine & - & - \\
\hline L-Arginine & Matheson Coleman \& Bell & - \\
\hline DL-Serine & - & - \\
\hline L-Threonine & - & - \\
\hline DL-Valine & - & - \\
\hline L-Tryptophan & - & - \\
\hline DL-Tyrosine & - & - \\
\hline Aniracetam & \multirow{8}{*}{ NOOTROPICS DEPOT } & - \\
\hline Coluracetam & & - \\
\hline Fasoracetam & & - \\
\hline Phenylpiracetam & & - \\
\hline Pramiracetam & & - \\
\hline Noopept & & - \\
\hline$\alpha$-GPC & & - \\
\hline CDP-Choline & & - \\
\hline
\end{tabular}




\subsection{Structures}

\subsubsection{Organic Moieties Used for Surface Modification}

\subsubsection{1-Eicosene (C20)}

1-eicosene $(\mathrm{C} 20)$ is an extended form of a popular C18 RP stationary phase by two carbons (Figure 18). Therefore, hydrophobicity of the resulting column is expected to be higher than typical monomeric C18 stationary phases. Aside from its strong RP properties, the silica hydride bed facilitates the $\mathrm{C} 20$ column to operate in ANP mode to retain polar analytes. In summary, C20 is predicted to achieve retention and separation of wide analyte polarity range.

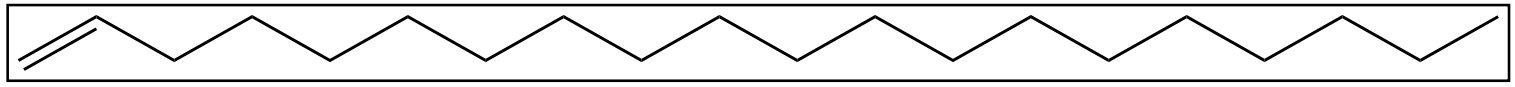

Figure 18. Structure of 1-eicosene (C20) Moiety.

\subsubsection{Allylpentafluorophenyl (PFP)}

Pentafluorophenylpropyl (PFP)-bonded stationary phases can be synthesized from hydrosilylation-mediated attachment of allylpentafluorobenzene (Figure 19) to the hydride surface. The PFP column has been a widely used column in pharmaceutical industries due to its ability to exhibit numerous column-analyte interactions: $\pi-\pi$ interaction, H-bonding, hydrophobic interaction, dipole-dipole, and cation exchange [41, 42]. This feature is often referred as the mixed mode [43, 44], and it allows for the unique analyte retention and selectivity. One study by Bell et al. (2005) suggests that PFP exhibits "U-shaped" retention profile depending on the mobile phase conditions [45], which resembles the retention profile shown by the silica hydride materials. Since many drug molecules have been engineered to have optimum membrane permeability 
and solubility, they typically contain both hydrophobic constituents such as aromatic ring, as well as polar functional group. A ligand like PFP allows for intermolecular interactions in multiple aspects, therefore it is an ideal choice of stationary phase for drug analyses.

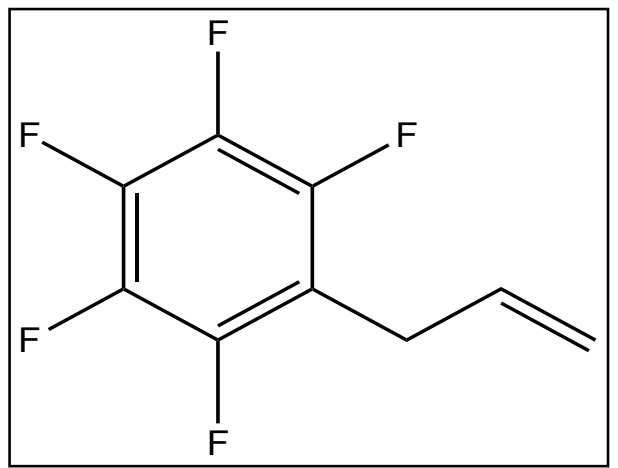

Figure 19. Structure of Allylpentafluorobenzene (PFP) Moiety.

\subsubsection{Allyloxy-DL- $\alpha$-tocopherol (TOCO)}

Tocopherol is a physiologically active fat-soluble compound known as Vitamin E. The role of this molecule is not completely understood. Many studies indicate its primary function as a radical scavenger to protect cell membranes from oxidative damage. In the meantime, other studies hypothesize its activity also as a gene regulator and enzyme inhibitor [46-48].

Allyloxy-DL- $\alpha$-tocopherol (TOCO) used in this study is a synthetic derivative of tocopherol known as DL- $\alpha$-tocopherol (all-rac- $\alpha$-tocopherol), which consists of all eight possible diastereomers, or four pairs of racemates (Figure 20). Of all tocopherol isomers, the $\mathrm{D}$ isomer (RRR- $\alpha$-tocopherol) is the only form found in nature and considered more bioactive [49-51]. Although enantiomerically pure $\alpha$-tocopherol olefin is the most ideal candidate for providing chromatographic selectivity for biomolecules, the DL olefin was 
used instead due to availability reasons. Based on its structure, the resulting column would likely interact with analyte molecules by H-bonding, hydrophobic interaction, and $\pi-\pi$ interaction. Overall, a DL-TOCO phase is expected to exhibit unique analyte selectivity.

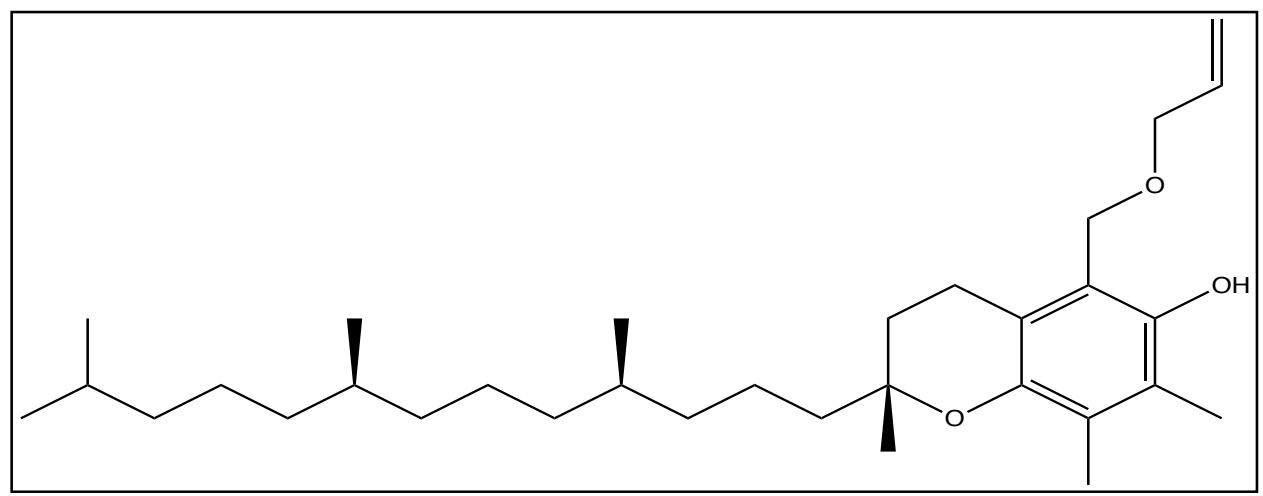

Figure 20. Structure of Allyloxy-DL- $\alpha$-tocopherol (TOCO) Moiety.

\subsubsection{10-undecenyloxycarbonyl-leucine (LEU)}

10-undecenyloxycarbonyl-leucine (LEU), also known as leucine surfactant, consists of an eleven-carbon alkyl chain esterified to a carboxyl derivative of amino acid leucine (Figure 21). This molecule was originally developed and donated by Dr. Shamsi at Georgia State University as a chiral recognition ligand in electrokinetic capillary chromatography (EKC) $[52,53]$. Most of the chiral separation in EKC has been demonstrated by dipeptide polymeric surfactants. Though, monomeric LEU surfactant alone is also hypothesized to show chiral recognition to some extent in HPLC applications.

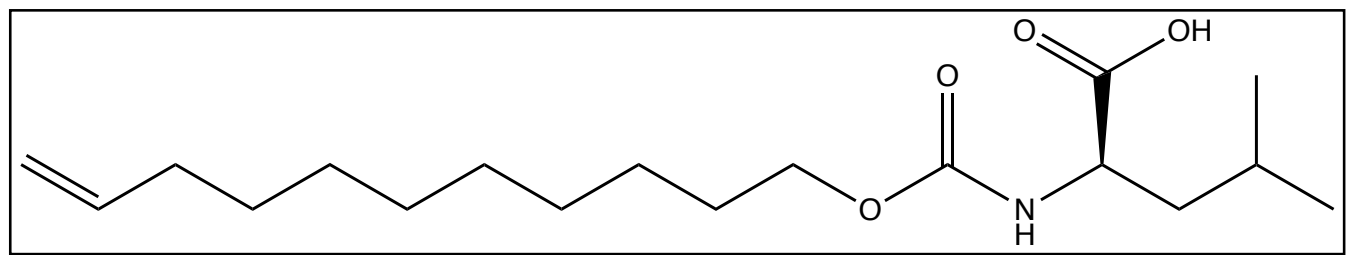

Figure 21. Structure of N-undecanoxycarbonyl-leucinate (LEU) Moiety. 


\subsubsection{Analytes Used for Column Evaluation}

\subsubsection{SRM 870}

Standard Reference Material ${ }^{\circledR} 870$ (SRM 870) developed by NIST is a mixture containing five organic molecules (Figure 22) that can be used to evaluate the general chromatographic performance of HPLC columns. This testing mixture is typically used for the characterization of C18 RP columns.

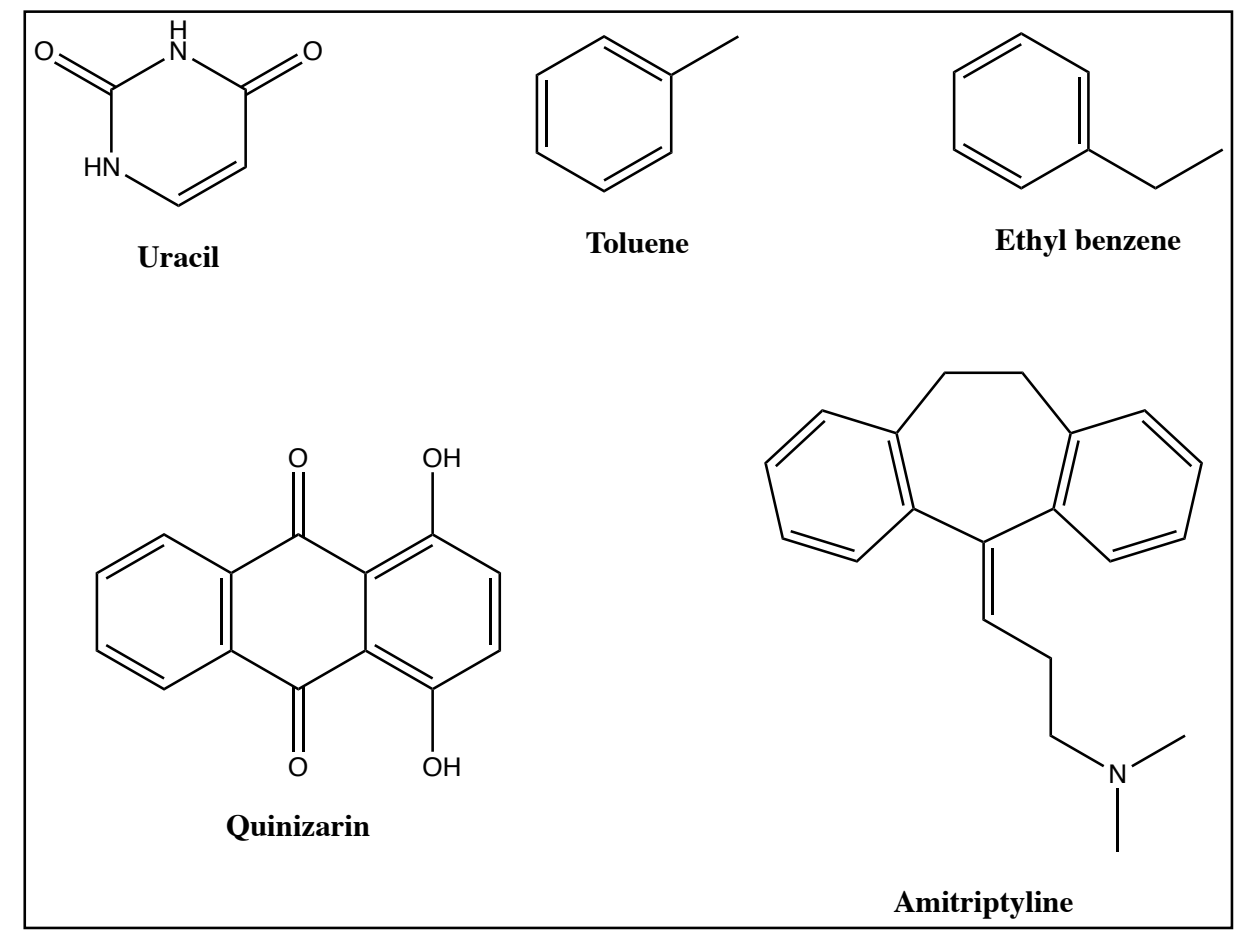

Figure 22. Five Analytes in SRM 870 Mixture.

In this study, however, the mixture was used to compare the newly developed columns in five criteria: void volume, methylene selectivity, hydrophobic retention, metal activity, and silanol activity. The void volume was estimated by using uracil as an unretained "void" marker. Toluene and ethyl benzene are two structurally similar nonpolar molecules used to evaluate hydrophobic retentiveness and methylene selectivity. Quinizarin is a chelator agent and eluted as a tailing peak when metal impurities are 
present within a column and have high activity towards the analyte molecules. Lastly, amitriptyline is a basic analyte and high silanol activity also results in severe peak tailing.

\subsubsection{Neue Test}

One of the two Neue test was performed to calculate for the hydrophobicity parameter of the columns [54]. The testing mixture contains uracil, naphthalene, and acenaphthene (Figure 23). Second half of the test, the silanophilicity test, was not performed since silanol activity is measured by MicroSolv QC test and SRM 870 test mixture.
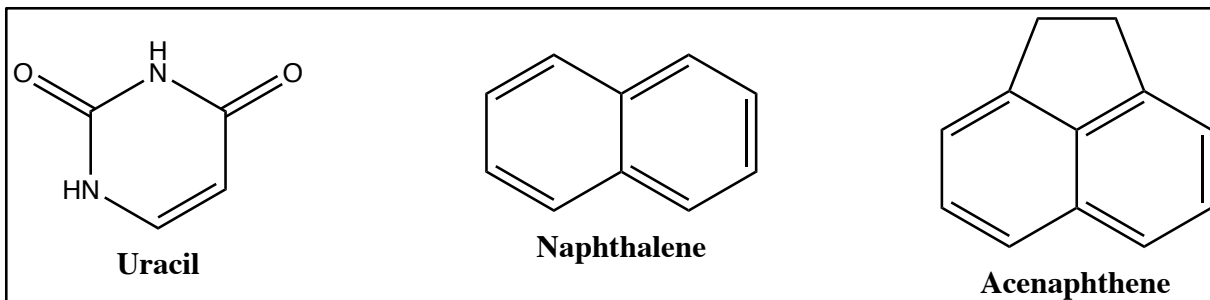

Figure 23. Compounds Used in Neue Test.

\subsubsection{Dimethoxybenzene (DMB) Selectivity}

Selectivity for anisole and three (o-, m-, p-) dimethoxybenzenes (DMB) were evaluated on a PFP column and compared against a Phenyl Hydride column (Figure 24).

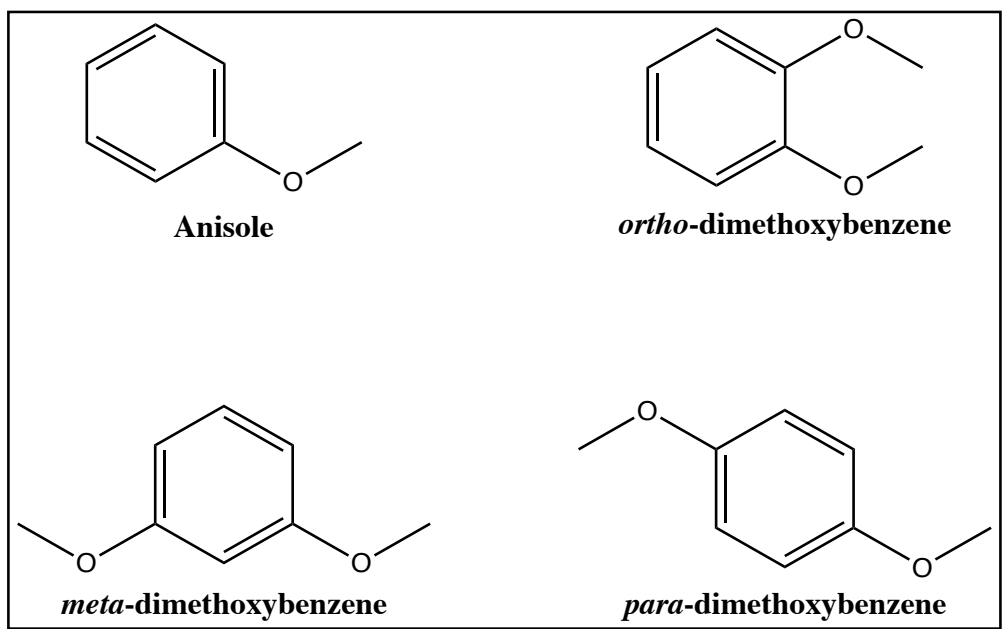

Figure 24. Structures of Anisole and Three Dimethoxybenzenes. 


\subsubsection{Dual Retention Capabilities}

Dual retention capabilities of newly developed bonded phases were characterized by using polar, nonpolar, and amphipathic molecules: adenine (or adenosine), glyburide, and caffeine, respectively (Figure 25).

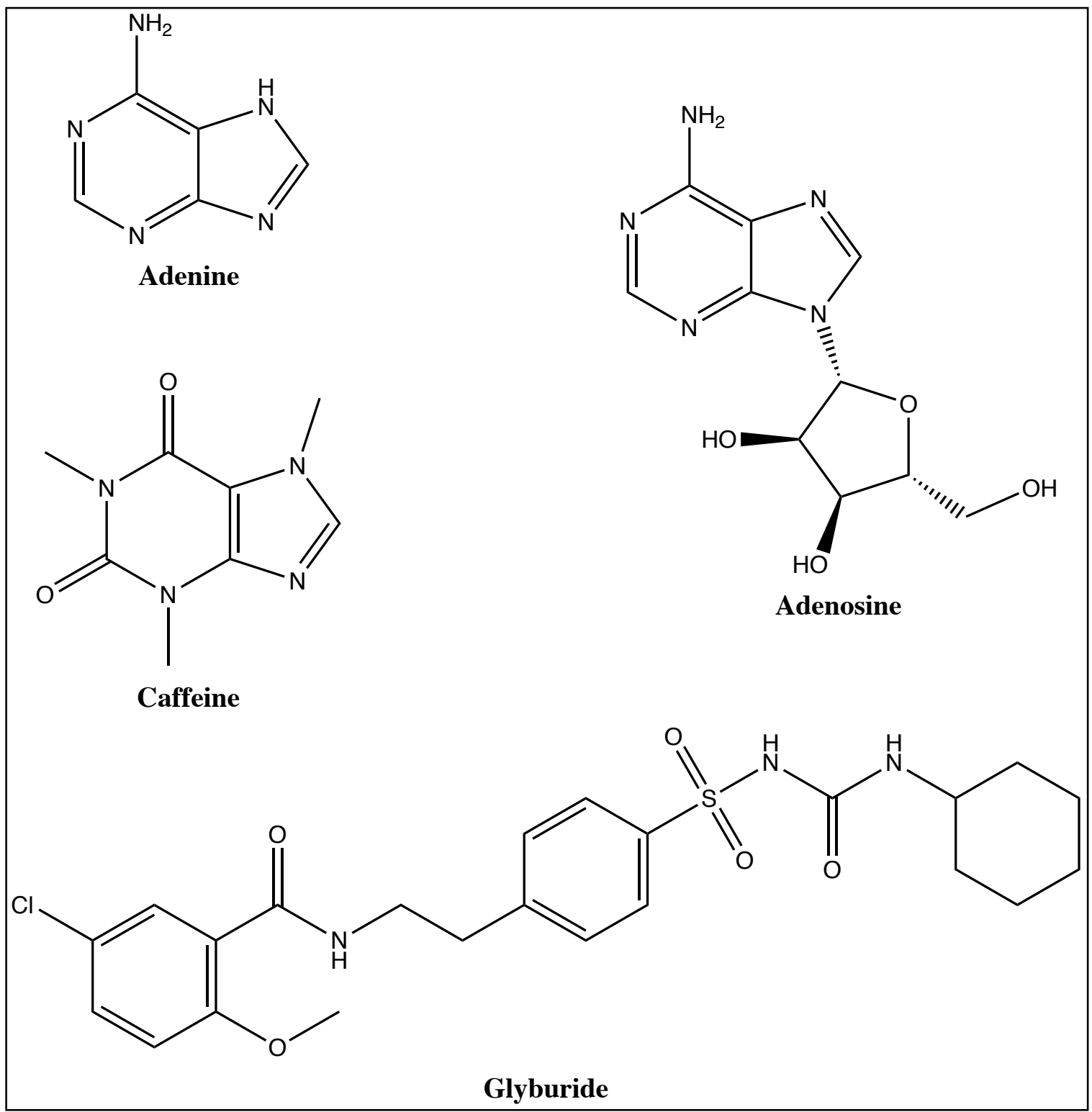

Figure 25. Compounds Used to Evaluate Dual Retention Properties. 


\subsubsection{Analytes Detected in Demonstration Studies}

Structures of the analytes of interest are shown in Figures 26 for USDA grape skin analysis, Figures 27-29 for drug bioanalysis, and Figure 30 for the ANP-HDX-MS project.<smiles>Oc1cc(O)c2c(c1)OC(c1ccc(O)c(O)c1)C(O)C2</smiles>

Catechin/Epicatechin<smiles>O=c1c(OC2O[C@H](CO)[C@@H](O)[C@H](O)[C@H]2O)c(-c2ccc(O)c(O)c2)oc2cc(O)cc(O)c12</smiles>

Quercetin 3-glucoside<smiles></smiles><smiles>O=c1c(OC2O[C@H](CO)[C@@H](O)[C@H](O)[C@H]2O)c(-c2ccc(O)cc2)oc2cc(O)cc(O)c12</smiles>

Kaempferol 3-glucoside

Figure 26. Structures of Standards Used in Grape Metabolomics Analysis. 


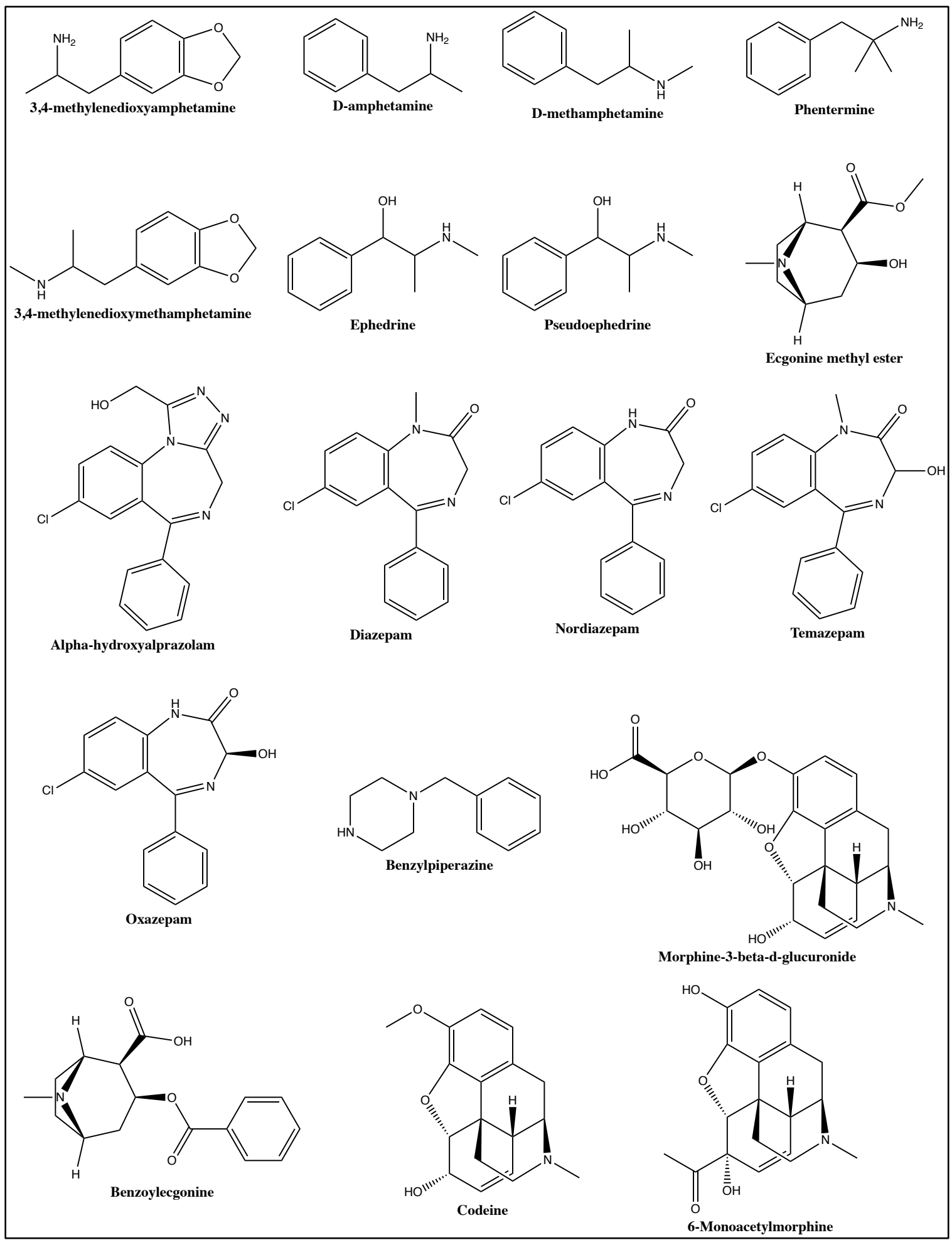

Figure 27. Structures of Common Drugs of Abuse. 


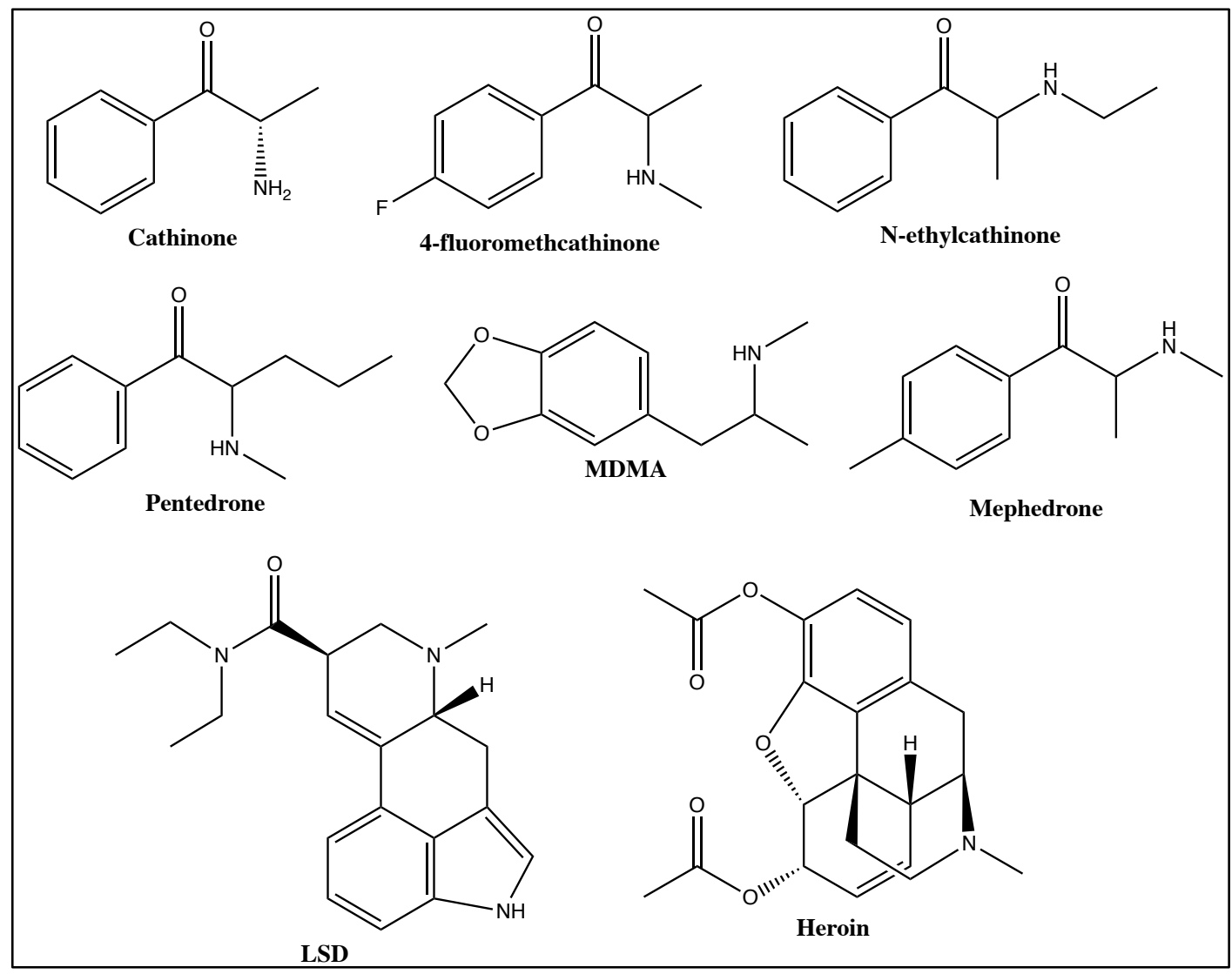

Figure 28. Structures of Illicit Substances Detected in Neat Solution.

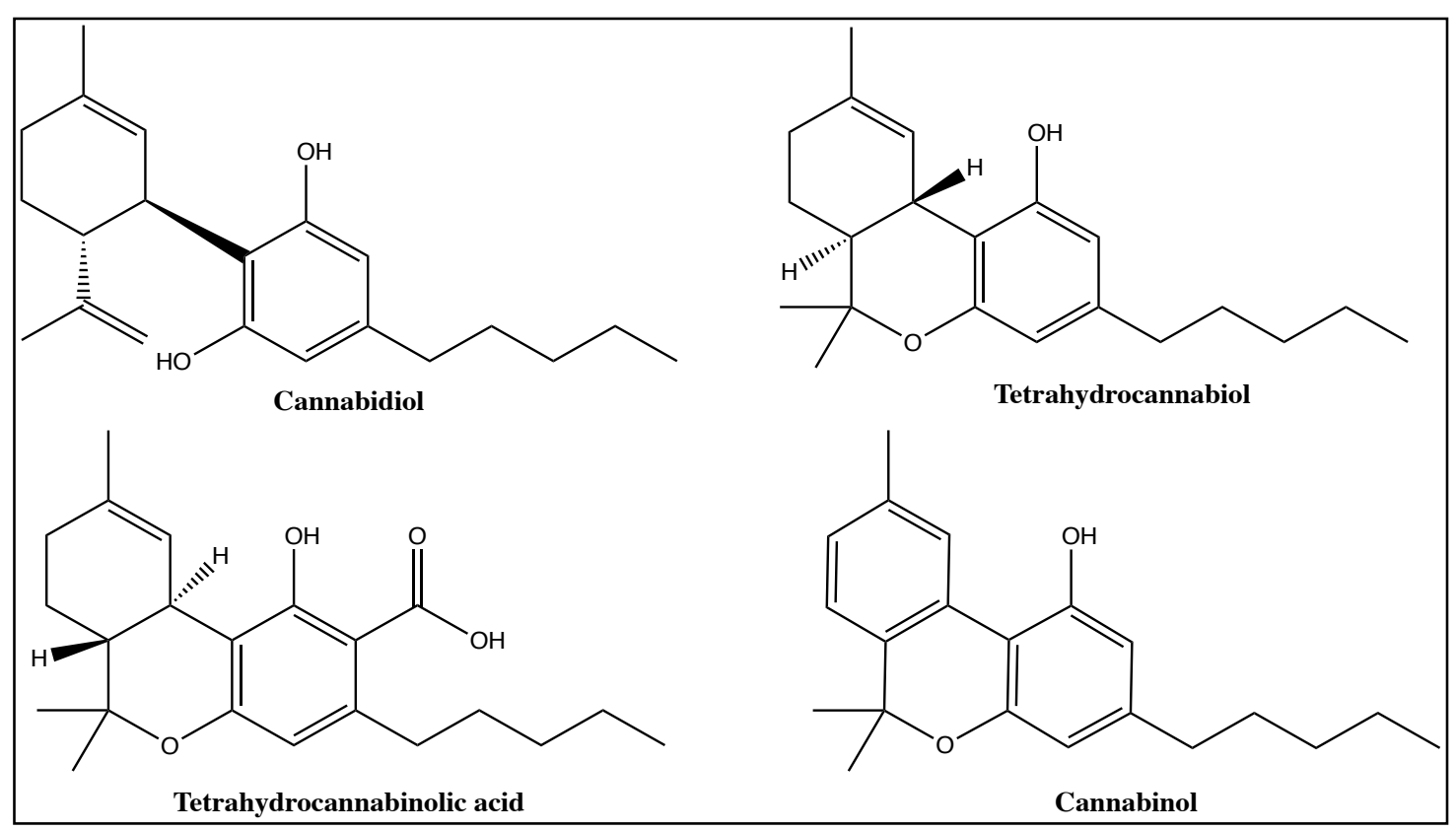

Figure 29. Structures of Phytocannabinoids of Interest. 


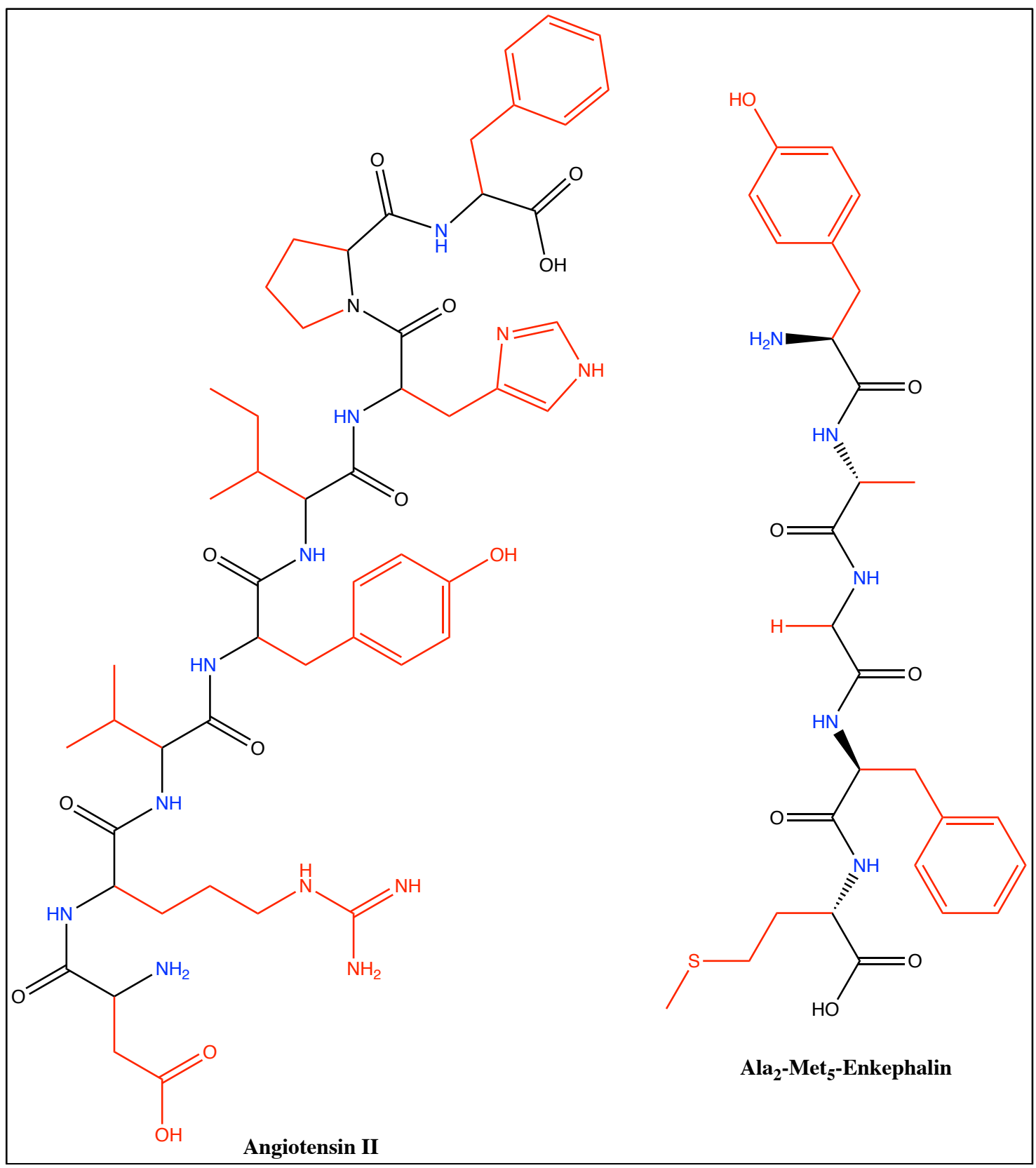

Figure 30. Structures of Two Model Peptides Used in ANP-HDX-MS Study.

\subsubsection{Selectivity Test}

Groups of compounds used for the column selectivity assessment were steroids

(Figure 31), amino acids (Figure 32), neurotransmitters (Figure 33), nucleobases (Figure 34), nootropics (Figure 35), sugars (Figure 36), and vitamins (Figure 37). 


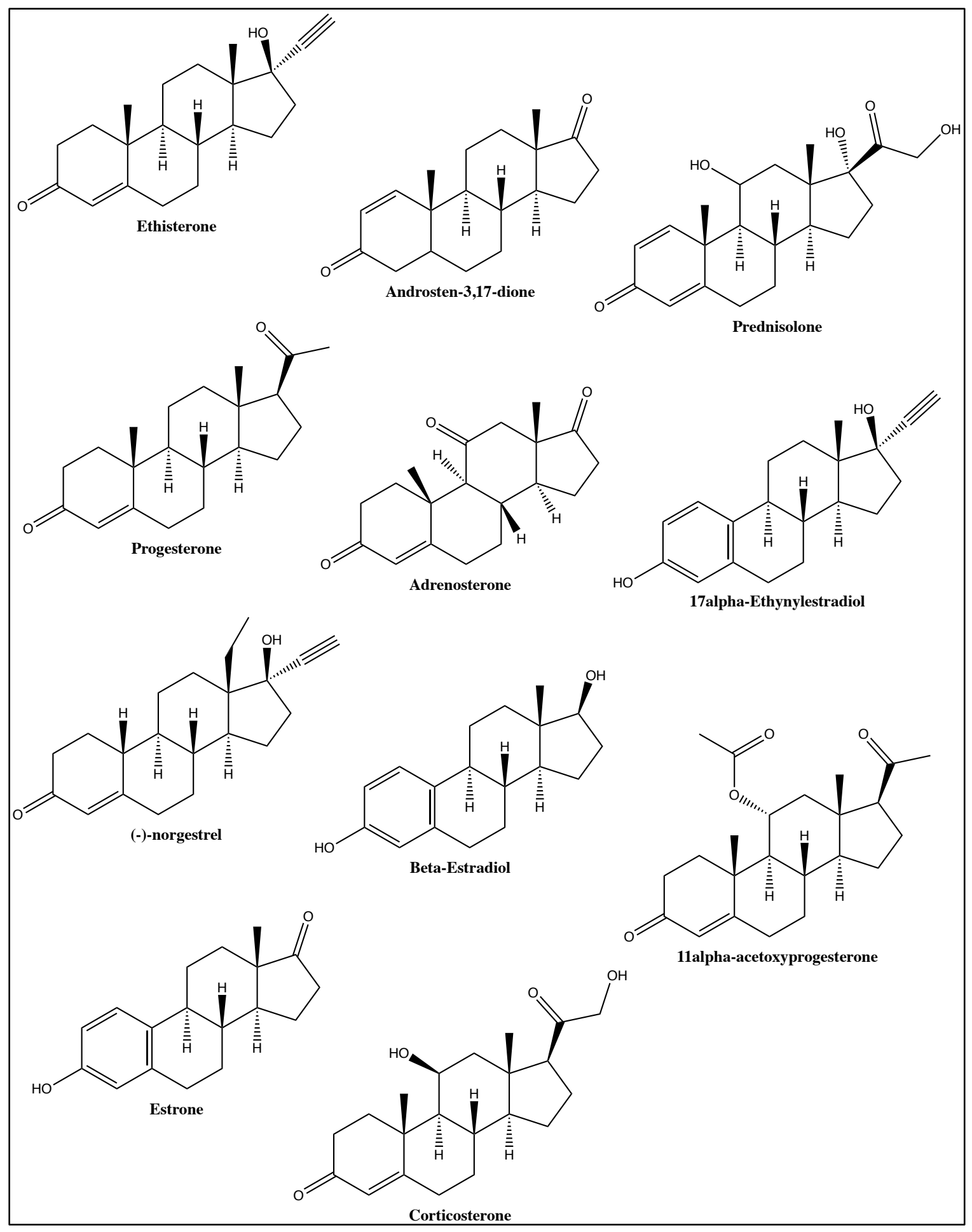

Figure 31. Structures of Steroids. 


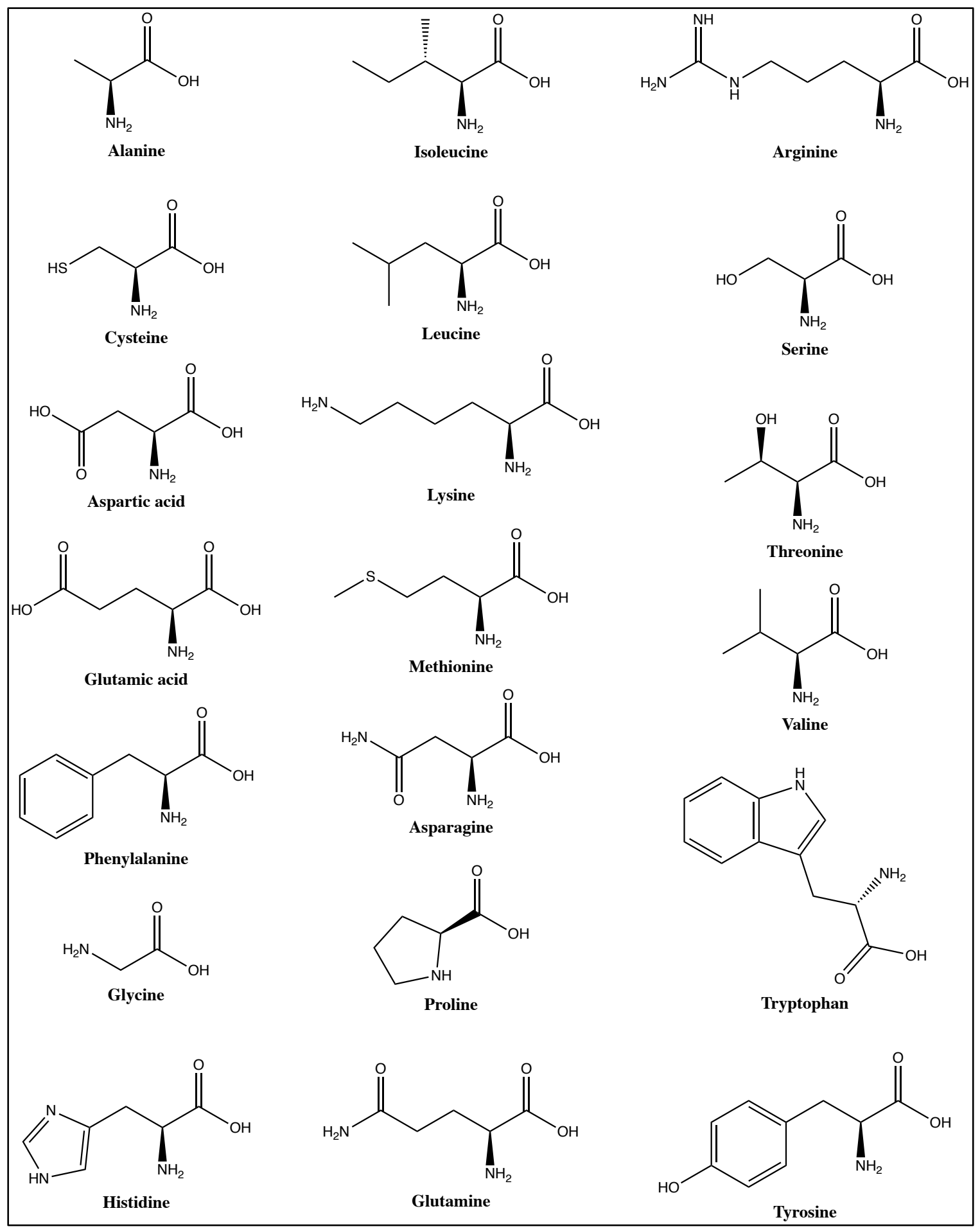

Figure 32. Structures of Amino Acids. 


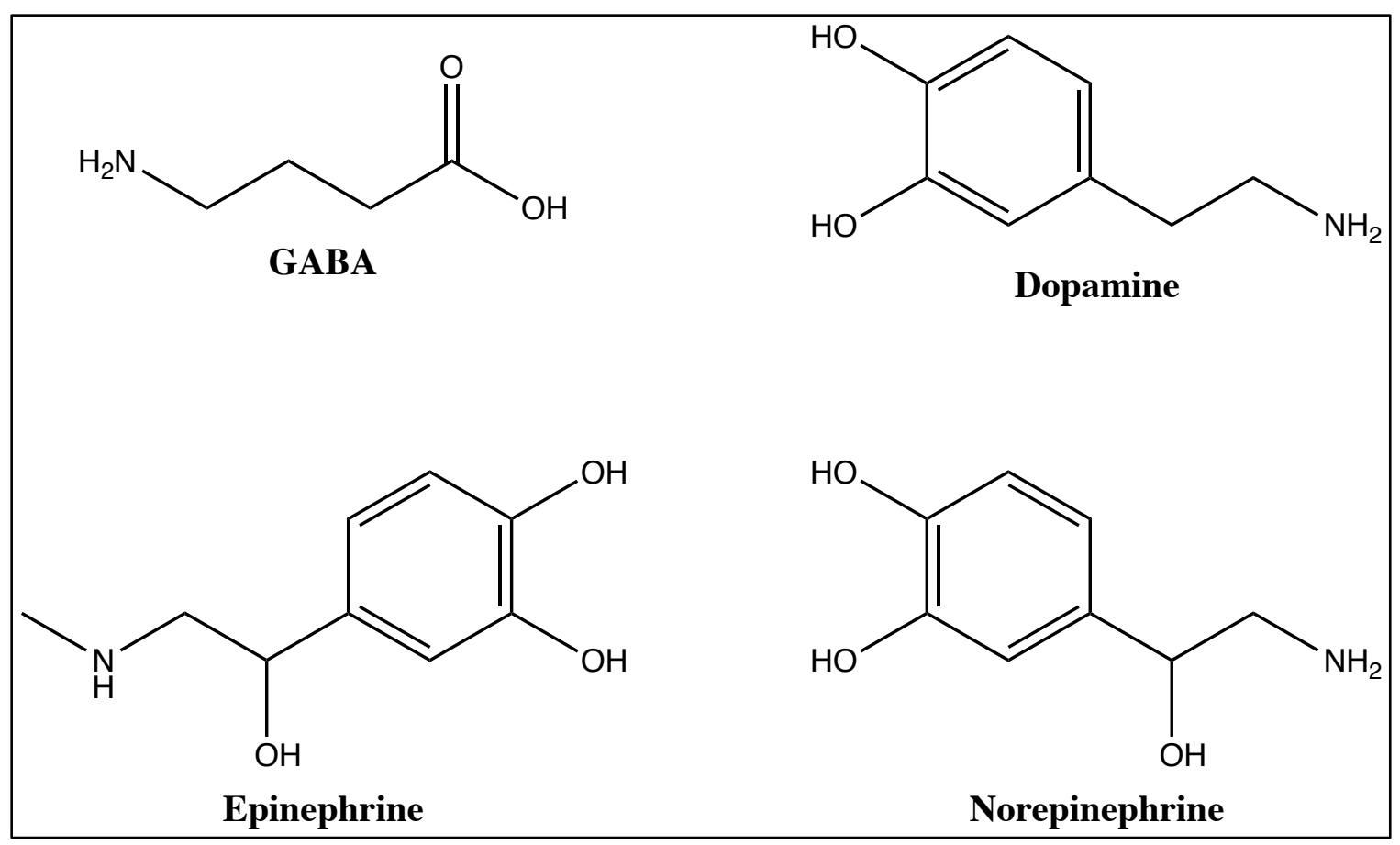

Figure 33. Structures of Neurotransmitters.

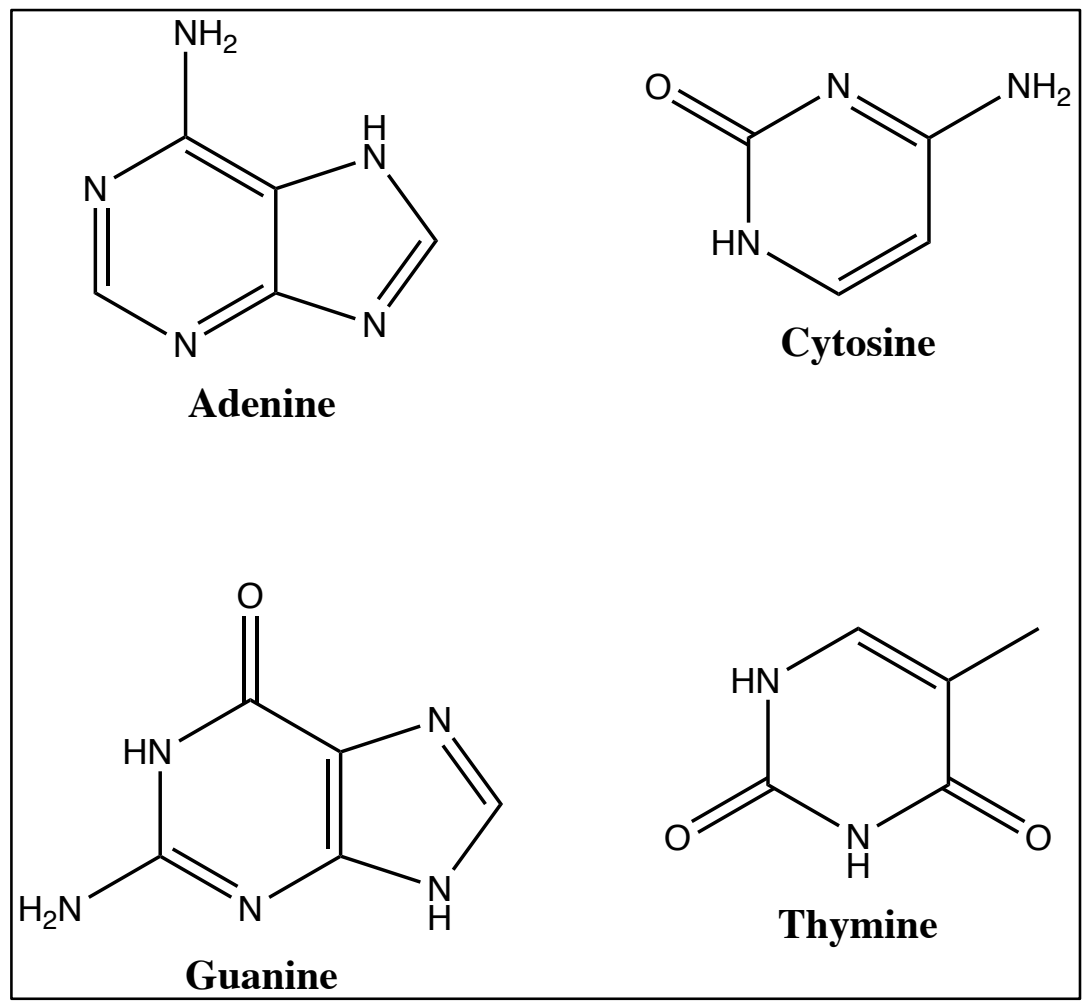

Figure 34. Structures of Nucleobases. 


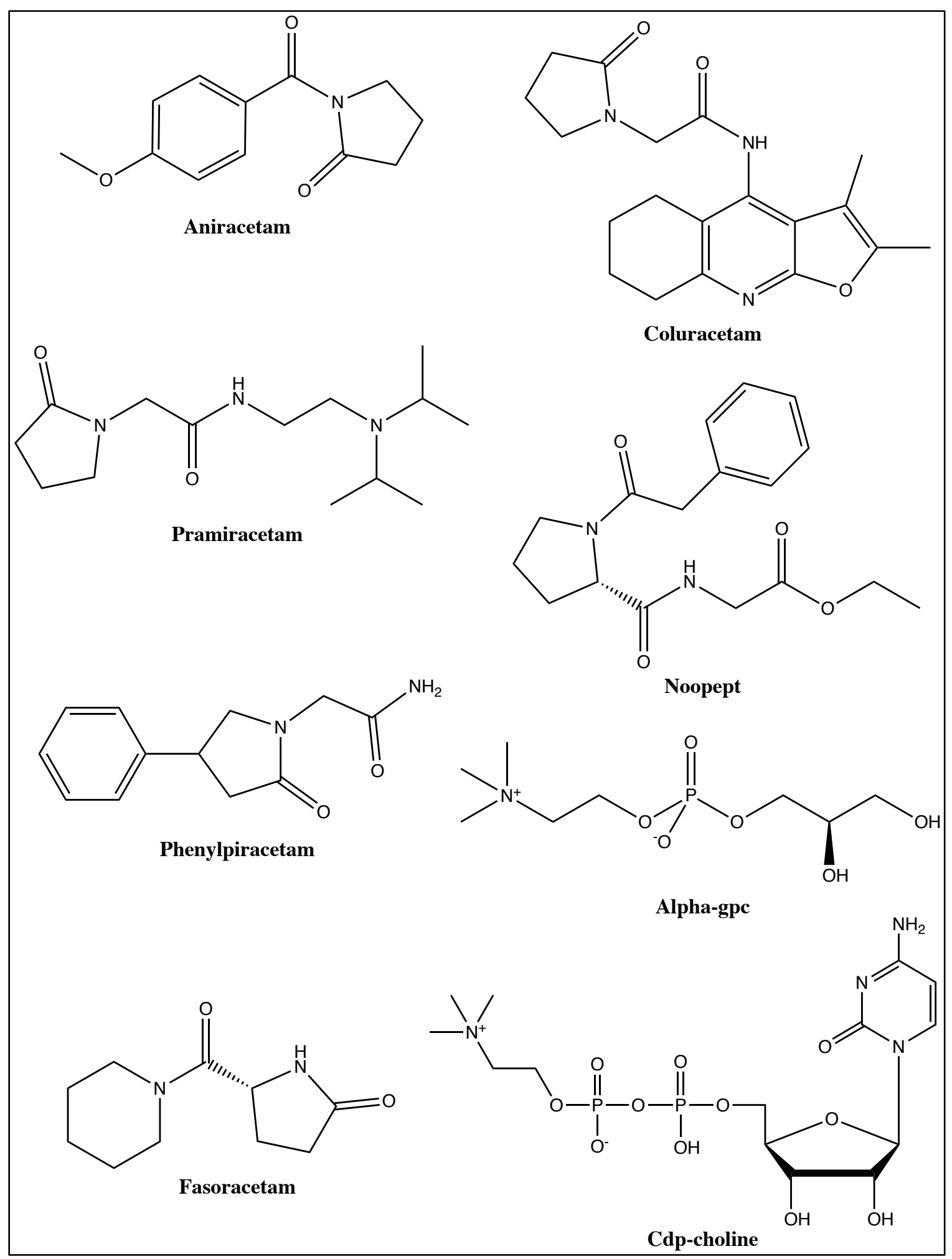

Figure 35. Structures of Nootropics. 


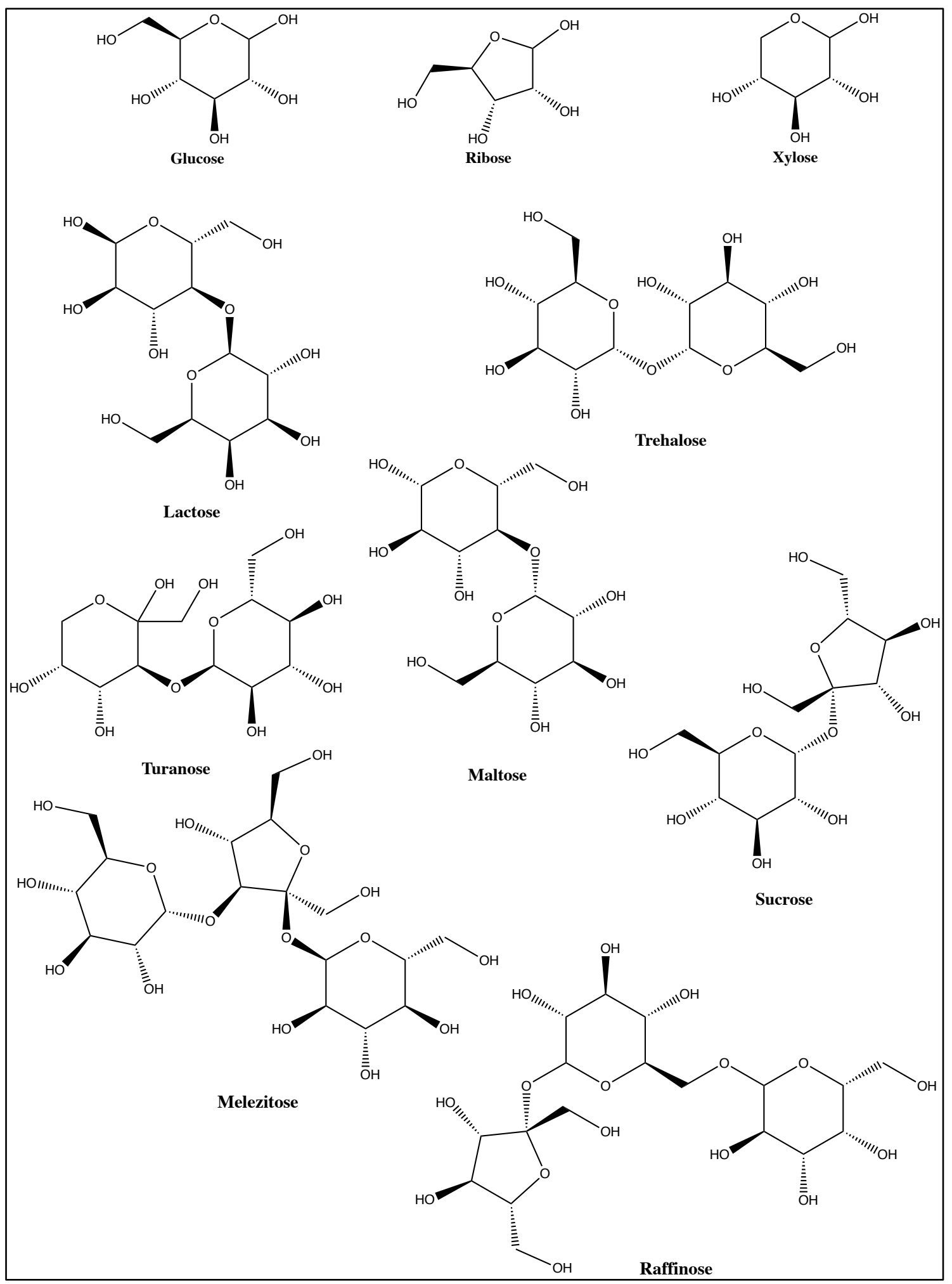

Figure 36. Structures of Sugars. 


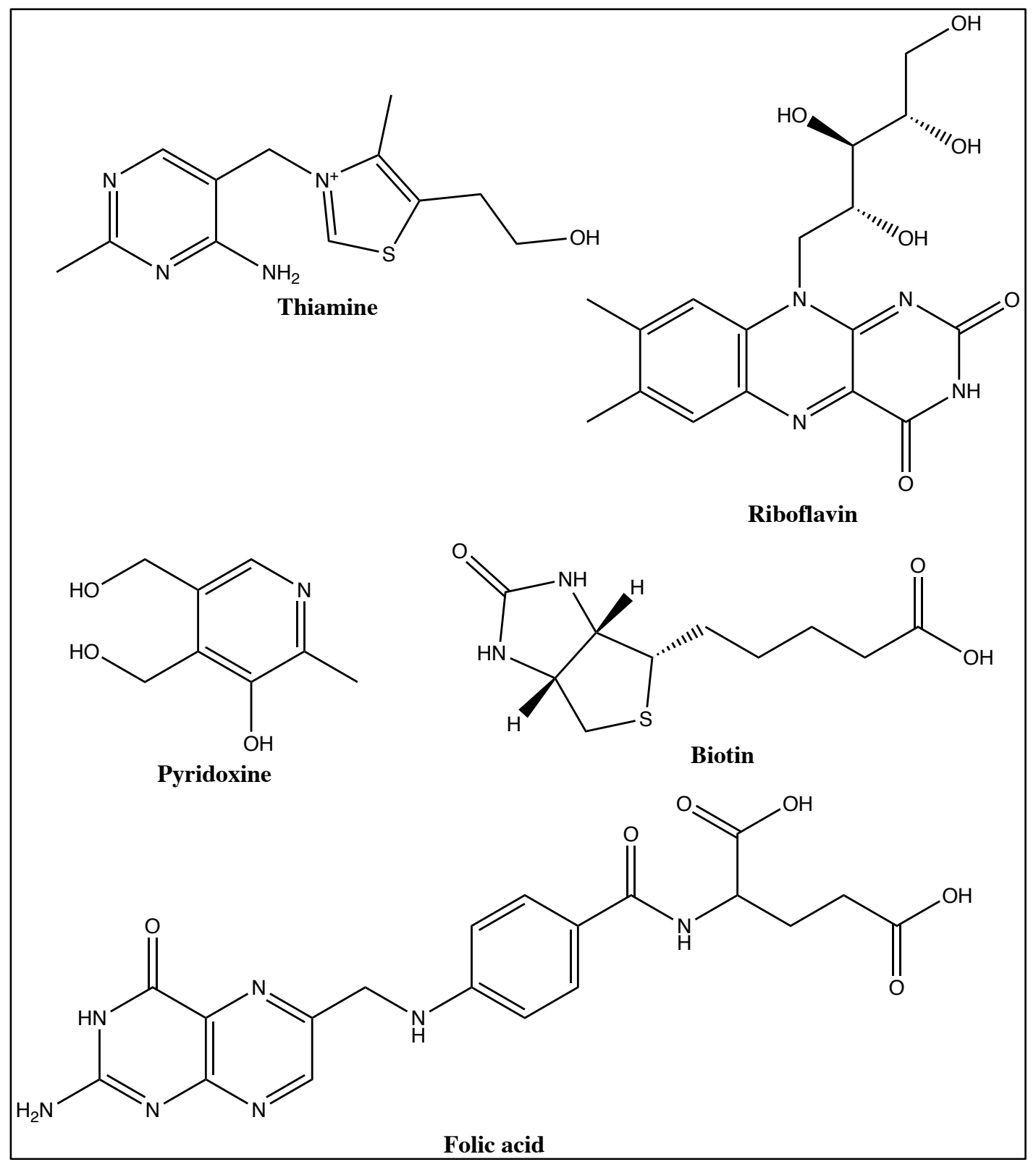

Figure 37. Structures of Vitamins.

\subsection{Methods}

\subsubsection{Molecular Dynamics (MD) - Hydride Surface Simulations by MMFF94}

Another objective in this study is to better understand the surface behavior of silica hydride and its relevance to the unique characteristics observed in the chromatographic experiments. One way to achieve this goal is by utilizing molecular dynamics (MD) 
simulation, a technique to computationally visualize and predict physical movements of atoms and molecules. Quantum mechanics (QM) is considered as a comprehensive way of performing this type of simulation; however, QM is often avoided due to the massive amount of calculations, time, and cost it requires. Molecular mechanics (MM) is a less detailed approach than QM but requires less resources and time. Therefore, MM is suitable alternative for the computational modeling of large molecular systems such as proteins [55].

MM calculation is based upon several assumptions: (1) treating the nucleus and electrons around them as spheres, (2) treating bonds between atoms/molecules as springs, (3) using experimental parameters such as force constants and equilibrium for some of the potential energy functions, and (4) the potential energy of a system as the summation of individual energy terms (Eq. 2.3.1.1-2.3.1.3). These potential energy functional forms are known as an interatomic potential, or a force field.

$$
\begin{gathered}
E_{\text {TотAL }}=E_{\text {covalent }}+E_{\text {noncovalent }} \\
E_{\text {covalent }}=E_{\text {bond }}+E_{\text {angle }}+E_{\text {dihedral }} \\
E_{\text {noncovalent }}=E_{\text {electrostatic }}+E_{\text {van der Waals }}
\end{gathered}
$$

In this study, Merck Molecular Force Field 94 (MMFF94) [56] was utilized to briefly simulate the surface behavior of silica hydride and ordinary silica for comparison. Segments of silica particles (48 Si atoms) were designed on ChemDraw (PerkinElmer). The generated silica particles were structurally optimized in the aqueous media (224 water molecules) and energy minimization was performed on Chem3D (CambridgeSoft) using MMFF94. Parameters and conditions used are shown in Table 11. 
Table 11. Parameters Used for the MD Simulations of Silica in Aqueous Media.

\begin{tabular}{|c|c|}
\hline Si Atoms & 48 \\
\hline H$_{2} \mathbf{O}$ (Molecules) & 224 \\
\hline Minimum RMS Gradient & 0.001 \\
\hline Step Interval (fs) & 1 \\
\hline Frame Interval (fs) & $1,000,000,000$ \\
\hline Terminate After (steps) & $1,000,000,000$ \\
\hline Heating/Cooling Rate (kcal/atom/ps) & 100 \\
\hline Target Temperature (K) & 298 \\
\hline
\end{tabular}

\subsubsection{Synthetic Procedures - Hydrosilylation}

All glassware used in this procedure was oven-dried overnight at $100^{\circ} \mathrm{C}$. Bare silica hydride materials were also stored and dried overnight at $120^{\circ} \mathrm{C}$ under the vacuum $(-25$ inHg). Speier's catalyst was prepared by dissolving 1 gram of $\mathrm{H}_{2} \mathrm{PtCl}_{6} \bullet 6 \mathrm{H}_{2} \mathrm{O}$ in 244 $\mathrm{mL}$ of 2-propanol to give $10 \mathrm{mM}$ concentration. The Pt catalyst must first undergo an induction period, where both olefin $(0.008 \mathrm{~mol} / \mathrm{gram}$ of silica hydride $)$ and the catalyst $(1: 10$ - molar ratio of olefin to the catalyst $)$ in the toluene media $(1: 20 \mathrm{w} / \mathrm{v}$ ratio - silica to toluene) are heated for 30 minutes at $70^{\circ} \mathrm{C}$. Here, $\mathrm{Pt}(\mathrm{IV})$ is catalytically activated by reduction to lower-valent states such as $\mathrm{Pt}(\mathrm{II})$ and $\mathrm{Pt}(0)$ colloids, which is believed to be the cause of continuous color change that one may observe in the reaction media [57]. Once the temperature stabilizes, the reaction remains for another 1 hour, followed by the gentle addition of silica hydride. The temperature is then raised to $100^{\circ} \mathrm{C}$ and held for 96 up to 100 hours.

Upon completion, the reaction product is separated from the toluene media using a $90 \mathrm{M}$ sintered glass funnel followed by $3 \times 25 \mathrm{~mL}$ hot toluene wash at $90{ }^{\circ} \mathrm{C}$, and $2 \times 25$ 
$\mathrm{mL}$ wash with diethyl ether. For the PFP phase, the product was washed with $50 \mathrm{~mL}$ instead of $25 \mathrm{~mL}$ volume and an additional $50 \mathrm{~mL}$ dichloromethane wash step was performed between toluene and diethyl ether washes. Then, products are dried under a fume hood at room temperature for overnight to remove the residual diethyl ether. Complete removal of excess solvents was achieved by vacuum drying at $110^{\circ} \mathrm{C}$ for 24 hours. The amounts of each reagent used are shown in Table 12. The reaction setup for hydrosilylation is shown in (Figure 38).

Table 12. Amount of Materials Used for Hydrosilylation.

\begin{tabular}{|c|c|c|c|c|}
\hline & TOCO & PFP & C20 & LEU \\
\hline Amount of olefin used (g) & 17.0400 & 8.3525 & 11.2038 & 6.9900 \\
\hline Silica hydride (g) & 5.0076 & 5.0016 & 5.0030 & 2.6630 \\
\hline Toluene (mL) & $\sim 100$ & $\sim 100$ & $\sim 100$ & $\sim 53.3$ \\
\hline 10 mM Speier's Cat. (mL) & 0.976 & 0.976 & 0.976 & 0.520 \\
\hline \hline Toluene (90'C) wash (mL) & $3 \times 25$ & $3 \times 50$ & $3 \times 25$ & $3 \times 25$ \\
\hline $\begin{array}{l}\text { Dichloromethane wash (mL) } \\
\text { Ethyl ether wash (mL) }\end{array}$ & $3 \times 25$ & $3 \times 50$ & $3 \times 25$ & $3 \times 25$ \\
\hline $\begin{array}{l}\text { Notes: } \\
\text { Olefin: 0.008 mol/gram of silica hydride } \\
\text { Toluene: } 1: 20 \text { wt./vol. ratio (silica hydride to toluene) } \\
\text { Catalyst: } 1: 10 \text { mol ratio - (olefin to catalyst) }\end{array}$ & \\
\hline \hline
\end{tabular}




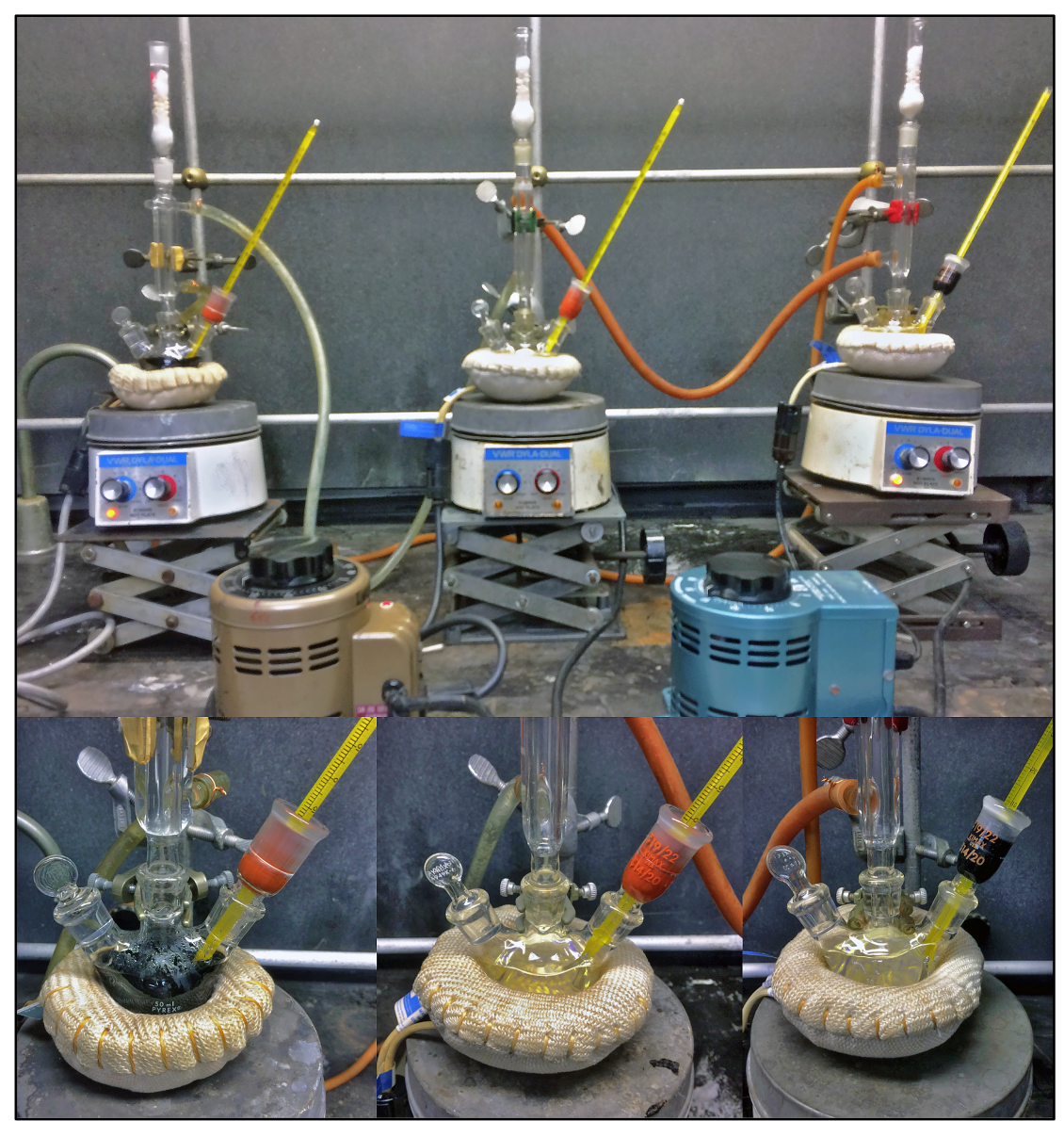

Figure 38. Setup for Hydrosilylation.

\subsubsection{Detection of PCBNs in Formulation and Cannabis Plant Extracts}

Small amounts of four cannabis plant samples were legally obtained from a local dispensary: "Super Silver Haze" (SSH), "Granddaddy Purple" (GDP), "White Fire OG" (WIFI), and "BIO". About $17 \mathrm{mg}$ of the plant leaves were weighed and added to $1 \mathrm{~mL}$ of extraction reagent $\left(50 \%\right.$ methanol in $\mathrm{DI}_{2} \mathrm{O}+0.1 \%$ formic acid) in a MagNA Lyser beads homogenization tube (Roche) and shaken on a Wig-L-Bug Amalgamator (Crescent) at maximum speed for about three minutes. The homogenate was diluted by 10 -fold and centrifuged to collect the supernatant. Then, sample solutions were filtered through 0.45 $\mu \mathrm{m}$ nylon membrane syringe filters and stored in amber RSA vials (MicroSolv 
Technologies, Inc.) until LC/MS injection.

Cannabidiol (CBD) formulation extract was prepared from KushyPunch CBD gummy edible (Caliva). Approximately $1.0 \mathrm{~g}$ of the formulation was weighed and dissolved in 5 $\mathrm{mL}$ of extraction solvent ( $50 \%$ methanol in $\mathrm{DI}_{2} \mathrm{O}+0.1 \%$ formic acid) and sonicated. The solution was heated to $70^{\circ} \mathrm{C}$ on a hotplate for about 15 minutes to allow for dissolution, followed by centrifugation at $1,294 \mathrm{x}$ g for 5 minutes to remove insoluble components. Supernatant was transferred into a $100 \mathrm{~mL}$ volumetric flask and filled to the line with DI $\mathrm{H}_{2} \mathrm{O}+0.1 \%$ formic acid. Then, the sample was loaded on a Bond Elut Certify II RP-SAX solid-phase extraction (SPE) cartridge after proper conditioning steps. The solid phase extraction (SPE) procedure was adopted and modified from Benvenuto et al., 2017 [58].

CBD and stable isotopically labelled CBD (CBD-d3) standards were obtained from Sigma Aldrich/Cerillant. Linearity of CBD was demonstrated in the range of $0.1-100$ $\mu \mathrm{g} / \mathrm{mL}$ using internal standard (IS) calibration and taking the CBD/CBD-d3 peak area ratio. A calibration curve was plotted and processed using GraphPad PRISM and Wolfram Mathematica. It is worth noting that the main objective of this project is neither method validation nor absolute quantification of phytocannabinoids. Rather, the goal is to briefly demonstrate the performance of the novel stationary phases for its potential applications in cannabis research.

\subsubsection{Retention Profile of Controlled Substances}

In addition to the cannabis project discussed in the previous section, the novel stationary phases were also evaluated for the analysis of controlled substances with/without the presence of biological matrix. 


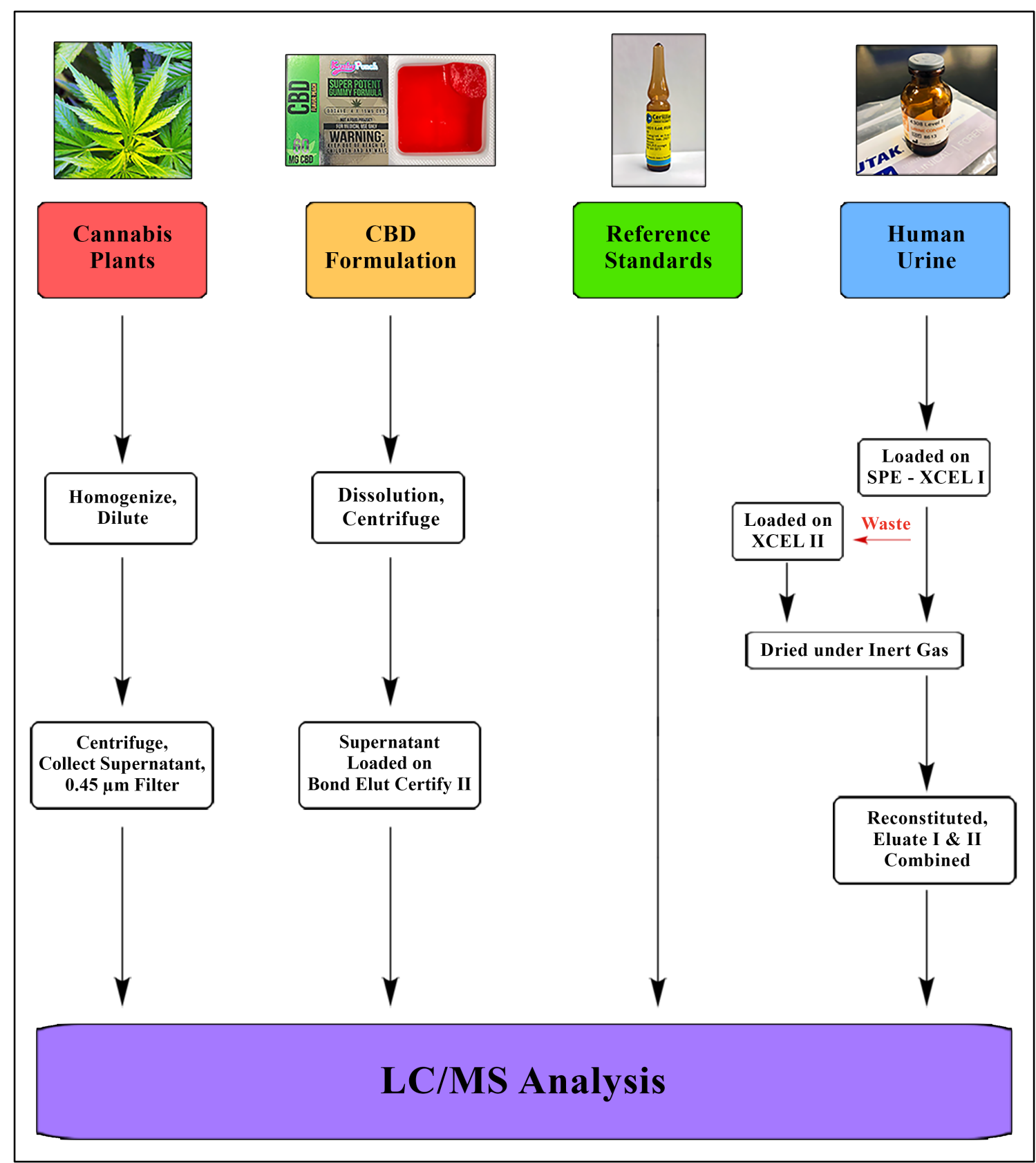

Figure 39. Sample Preparation Workflow for Controlled Substance Analyses.

The matrix used in this study was human urine obtained from UTAK (Level 1 Urine Toxicology Control), which contains eighteen controlled substances (shown in Figure 27, and tetrahydrocannabinolic acid from Figure 29). Sample preparation starts with reconstitution of lyophilized urine control in $10 \mathrm{~mL}$ DI $\mathrm{H}_{2} \mathrm{O}$, followed by $5 \mathrm{~mL}$ sample 
loaded on a UCT Clean Screen XCEL I SPE column to extract all substances except tetrahydrocannabinolic acid (THCA) [59]. THCA extraction is done by loading the XCEL I waste on an XCEL II SPE column. Both eluates from the SPE columns were dried under argon gas and combined after reconstitution with mobile phase solvents (Figure 39).

\subsubsection{Metabolomics Characterization of USDA Grape Cultivars}

Five dried grape skin samples were provided by Dr. Gary Takeoka (Albany, CA) and Dr. Craig Ledbetter (Parlier, CA) from the United States Department of Agriculture Agricultural Research Service (USDA-ARS). For sample extraction, approximately $1.0 \mathrm{~g}$ of ground grape skin powder were mixed with $15 \mathrm{~mL}$ of extraction reagent $(50 \%$ methanol $+48.5 \%$ DI $\mathrm{H}_{2} \mathrm{O}+1.5 \%$ formic acid), followed by vortex and sonication. After 5 minutes of centrifugation at 1,294 g, the supernatant was vacuum-filtered with a $0.45 \mu \mathrm{m}$ nylon membrane (MicroSolv). Twelve analytical standard stock solutions were prepared at $1 \mathrm{mg} / \mathrm{mL}$ in DMSO and diluted to $10 \mu \mathrm{g} / \mathrm{mL}$ with the extraction solvent for LC/MS detection.

Chromatographic performance was assessed on the Agilent MassHunter Qualitative Analysis Software. Another goal of this project is to better understand the metabolic characteristics of five grape cultivars. Thus, statistical analysis of the metabolite abundance was performed using the Metabolomics Analysis and Visualization Engine (MAVEN) [60]. The comprehensive metabolite profile was elucidated using $\mathrm{R}$ programming [61] by performing dimensionality reduction of dataset. Ward's minimum variance method was utilized for data clustering in the generated heatmap representation. 


\subsubsection{Proton Back-exchange Reduction by ANP-coupled HDX-MS}

Hydrogen-deuterium exchange mass spectrometry (HDX-MS) is a powerful and versatile technique for the structural elucidation of proteins. Some of the applications include conformational dynamics assessment, determination of post-translational modification sites and protein-protein/protein-ligand interaction sites [62]. Unlike other structural proteomics techniques such as NMR and X-ray crystallography, HDX-MS does not require a crystallization step or have severe limitations in molecular sizes and concentrations. However, HDX-MS has one critical drawback known as the "backexchange" (BE) and numerous methods have been proposed to minimize this undesirable effect. The concept behind this technique involves isotope-labeling of the sample proteins with deuterium by incubating in a $\mathrm{D}_{2} \mathrm{O}$ buffer. There are three types of hydrogens on a protein that exchange protons with the solvent. Although, alkyl $(\mathrm{C}-\mathrm{H})$ hydrogens exchange at extremely slow rate while the hydrogens on an acidic/basic/polar side chains exchange too rapidly; therefore, only the peptide amide $(-\mathrm{NH})$ backbone is monitored in the HDX-MS experiments. Solvent accessibility of the backbone amide plays a critical role in determining the exchange rate (from $<1$ second up to months); hence the incubation time required to exchange protons varies for each backbone amide $[63,64]$. Essentially, the proton BE phenomenon defeats the purpose of HDX experiment and must be minimized by strict control of sample environment during the exchange experiment and chromatographic procedures.

Typical workflow of the HDX-MS experiment is shown in Figure 40. The first step is the protein sample incubation in a $\mathrm{D}_{2} \mathrm{O}$ buffer solution, which induces H-D exchange 
in the order of most solvent accessible to the least. At different time-points (e.g., 0, 1, 5, $10,30 \ldots$ minutes), a small portion of the sample is aliquoted and transferred into the quenching solution to terminate the H-D exchange reaction. The quenching solution is at $0^{\circ} \mathrm{C}$ and $\mathrm{pH}$ of 2-3, which decreases the exchange rate by nearly 5 orders of magnitude compared to $\mathrm{pH} 7$ at $25^{\circ} \mathrm{C}$. The quenching solution also contains protease (e.g., trypsin or pepsin) which digests the sample proteins into peptide fragments so that mass difference caused by H-D exchange can be observed on a LC/MS instrument at peptide resolution. The proton BE initiates as soon as the protein is subjected to protiated solvents such as water in the quenching solution or aqueous mobile phase in the LC system. Even in strictly controlled setting with a refrigerated $\mathrm{LC}$ system at $0^{\circ} \mathrm{C}$ and $\mathrm{pH} 2, \mathrm{BE}$ can be as high as $20 \%$ of the entire protein in ESI-MS experiment $[63,65]$.

Over the course of years, numerous methods have been devised to minimize the $\mathrm{BE}$ effect in HDX experiments. Online column digestion is one of the common approaches today that results in reduced sample handling time. However, this method typically requires additional LC components such as trap column and switch valve, which could increase the complexity of operation. Furthermore, this approach serves as additional source of sample carryover which could potentially affect the measurement of peptide deuteration count [66].

In this study, silica hydride based-stationary phases and ANP chromatography are investigated as a novel strategy to minimize the BE in HDX-MS experiments. As of today, almost every proteomics applications utilize RP chromatography as the elution method. Unfortunately, RP requires larger amounts of aqueous solvent to facilitate 
retention and separation of the peptides by hydrophobic interactions. As demonstrated by Zhang et al., the composition of aqueous eluent and the length of exposure results in greater deuterium BE [67]. In this study, ANP chromatography is hypothesized to be better alternative to RP chromatography. This is because ANP retention is most efficient under high organic eluent condition, as discussed in Section 1.5.3. Thus, in theory ANP may achieve better separation and BE reduction simultaneously. In order to confirm this hypothesis, BE behavior during ANP elution was investigated by using two peptide models reconstituted in deuterium solution $\left(\mathrm{D}_{2} \mathrm{O}, 99.8 \% \mathrm{D}\right)$. Since the model peptides, $\mathrm{Ala}^{2}-\mathrm{Met}^{5}$-Enkephalin (EKP) and Angiotensin-II (A-II), are fully-deuterated and stored in $\mathrm{D}_{2} \mathrm{O}$ solution, $\mathrm{BE}$ occurs only upon their exposure to mobile phase after sample injection.

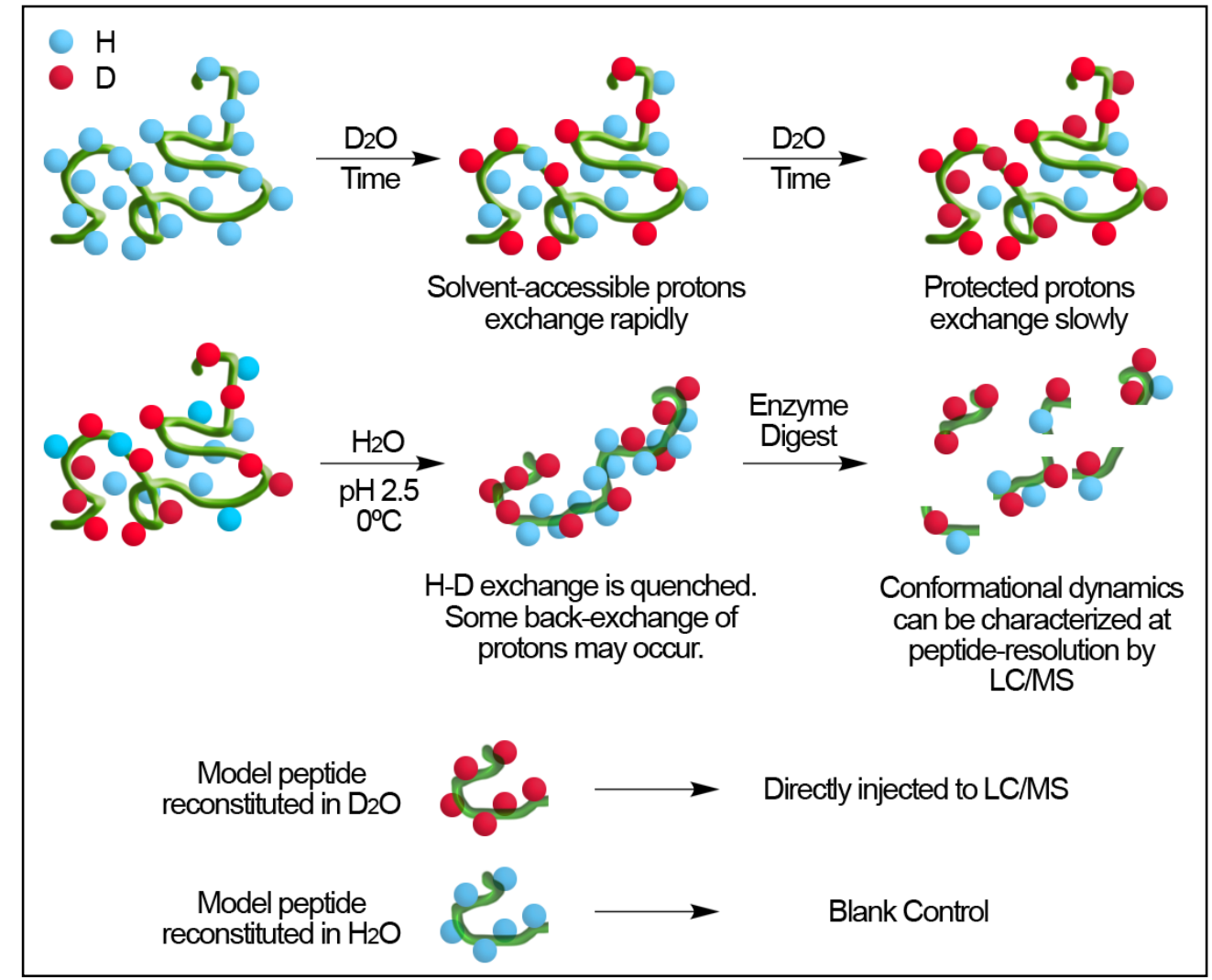

Figure 40. Scheme for Ordinary HDX-MS (Top) and ANP-HDX Evaluation (Below). 


\subsection{Instrumentation}

All instruments used in the study are listed in Table 13.

Table 13. List of Instruments Used in This Study.

\begin{tabular}{|c|c|c|}
\hline Instruments & Company & Cat.\#/Model \# \\
\hline HPLC 1200 Series - System 1 & \multirow{7}{*}{$\begin{array}{c}\text { Agilent } \\
\text { Technologies }\end{array}$} & - \\
\hline Degasser & & G1322A \\
\hline Quaternary Pump & & G1311A \\
\hline Thermostat for Autosampler & & G1330B \\
\hline High Performance Autosampler & & G1367B \\
\hline Thermostatted Column Compartment & & G1316A \\
\hline Diode Array Detector & & G1315D \\
\hline HPLC 1200 Series - System 2 & \multirow{6}{*}{$\begin{array}{c}\text { Agilent } \\
\text { Technologies }\end{array}$} & - \\
\hline Degasser & & G1379B \\
\hline Binary Pump & & G1312A \\
\hline High Performance Autosampler & & G1329A \\
\hline Thermostatted Column Compartment & & G1316A \\
\hline Diode Array Detector & & G1315D \\
\hline HPLC 1100/1200 Series \& 6200 Series MSD & \multirow{8}{*}{$\begin{array}{c}\text { Agilent } \\
\text { Technologies }\end{array}$} & - \\
\hline Degasser & & G1322A \\
\hline Binary Pump & & G1312B \\
\hline Thermostat for Autosampler & & G1330B \\
\hline High Performance Autosampler SL & & G1367C \\
\hline Thermostatted Column Compartment & & G1316A \\
\hline Isocratic Pump & & G1310A \\
\hline TOF Mass Detector & & G3250AA \\
\hline Nicolet 6700 FT-IR & \multirow{3}{*}{ Thermo Scientific } & 912A0751 \\
\hline Nicolet Avatar Smart Diffuse Reflectance & & $0028-497$ \\
\hline SMART iTR ATR Sampling Accessory & & $222-247000$ \\
\hline $\begin{array}{l}\text { Praying Mantis }{ }^{\mathrm{TM}} \text { Diffuse Reflection } \\
\text { Accessory }\end{array}$ & Harrick Scientific & DRP-XXX \\
\hline Praying Mantis ${ }^{\mathrm{TM}}$ Chamber & Harrick Scientific & HVC-DRM-5 \\
\hline Temperature Controller & Harrick Scientific & ATK-024-3 \\
\hline PE 2400 Series II CHNS/O Analyzer & PerkinElmer & $\mathrm{N} 2410650$ \\
\hline Avance III HD 300 WB NMR Spectrometer & Bruker & - \\
\hline
\end{tabular}




\subsubsection{Attenuated Total Reflectance (ATR)}

Attenuated total reflectance (ATR) is a form of Fourier-transform infrared spectroscopy (FTIR) based upon internal reflection of IR beam in ATR crystal, also known as the internal reflection element (IRE). A sample (e.g., solid/liquid) in close contact with IRE is subjected to IR light that has penetrated through the crystal. This light is known as the evanescent wave which penetrates through the sample and the incident light is reflected if no absorption occurs. Essentially, the detector measures the attenuated intensity of totally-reflected IR beam caused by sample absorption that may have occurred.

One advantage of ATR-FTIR is that it requires little to no sample preparation step for the most liquid and solid samples. This technique was used only for the characterization of liquid olefin materials. In this study, a Thermo Scientific Nicolet 6700 FT-IR equipped with a SMART iTR ATR Sampling Accessory and deuterated-triglycine sulfate (DTGS)-KBr detector was used. Parameters were set to scan from 525-4000 $\mathrm{cm}^{-1}$ range with a resolution of $4 \mathrm{~cm}^{-1}$ and 32 scans were collected and averaged.

\subsubsection{Diffuse Reflectance Infrared Fourier Transform Spectroscopy (DRIFTS)}

For the FTIR measurement of powder samples with rough surfaces, infrared spectra of diffuse reflection are collected by the technique called diffuse reflectance infrared Fourier transform spectroscopy (DRIFTS). Irradiation of the sample may result in specular reflection on the surface, while the remaining radiation may enter the bulk powder by refraction. The incident light that has entered the insides of powders is transmitted through them after some has been absorbed, or internally reflected at the 
powder surfaces; thereby, causing the radiation to diffusely reflect. Any diffuse reflected radiation must have transmitted through the powder particle at least once; therefore, a DRIFTS accessory is designed to collect the diffuse reflected radiation to generate a spectrum essentially equivalent to IR absorption spectrum of the sample.

Spectroscopic characterization by DRIFTS was employed on bare silica hydride and bonded phase products using the same FT-IR instrument equipped with the Nicolet Avatar Smart Diffuse Reflectance accessory. Parameters were set to scan from 400-4000 $\mathrm{cm}^{-1}$ range with a resolution of $4 \mathrm{~cm}^{-1}$, and 32 scans were averaged against pure $\mathrm{KBr}$ as a reference spectrum. All samples were prepared by diluting the silica material (5\% wt.) in a $\mathrm{KBr}$ matrix (95\% wt.) and grinding to fine powder to prevent excess light absorption.

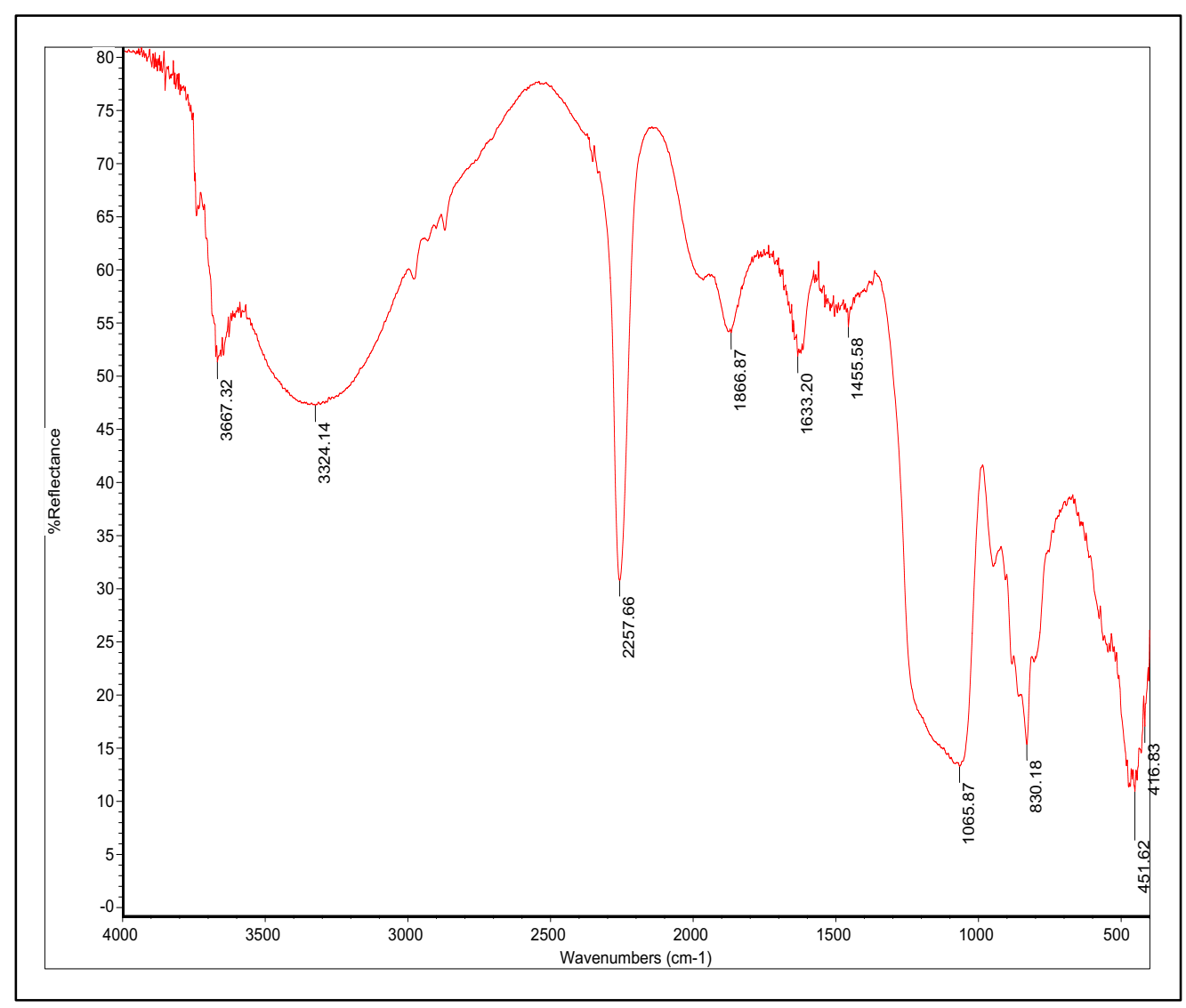

Figure 41. DRIFTS Spectra of Bare Silica Hydride. 
Absorption by the Si-H bond can be monitored by the sharp peak at $2250 \mathrm{~cm}^{-1}$ (Figure 41) $[68,69]$. Reacted bonded phases were compared against the ATR spectra to verify the presence of olefin moiety on the surface of silica hydride.

\subsubsection{Elemental Carbon Analysis}

Elemental analysis was employed for the determination of \% carbon in reacted products. The obtained values were utilized to calculate the density of bonded moieties on silica hydride surface by using the Berendsen-de Galan equation:

$$
\alpha_{R}=\frac{P_{C} \cdot 10^{6}}{S_{B E T} \bullet\left(100 \bullet M_{C} \cdot n_{C}-P_{C} \bullet M_{R}\right.}
$$

where $\alpha_{R}$ is surface coverage $\left(\mu \mathrm{mol} / \mathrm{m}^{2}\right), \mathrm{P}_{\mathrm{C}}$ as the $\%$ carbon, $\mathrm{S}_{\mathrm{BET}}$ as the specific surface area of the silica hydride $\left(400 \mathrm{~m}^{2} / \mathrm{g}\right), \mathrm{M}_{\mathrm{C}}$ as the atomic weight of carbon, $\mathrm{n}_{\mathrm{C}}$ as the number of carbon atoms found in the bonded organic moiety, and $\mathrm{M}_{\mathrm{R}}$ as the molecular weight of the organic moiety $[70,71]$.

The analysis was conducted by Galbraith Laboratories, Inc. (Knoxville, TN) with a PerkinElmer 2400 Series II CHNS/O Analyzer (PE-2400). Elemental carbon is indirectly quantified by detecting $\mathrm{CO}_{2}$ gas, which is produced by combusting 1-5 $\mathrm{mg}$ of the sample in pure oxygen at $920-980^{\circ} \mathrm{C}$ under static conditions. The PE-2400 instrument separates and analyzes the generated gas with a steady-state thermal conductivity analyzer.

\subsection{4 ${ }^{13} \mathrm{C}$ CP/MAS Solid-state NMR}

Carbon-13 solid-state nuclear magnetic resonance spectroscopy $\left({ }^{13} \mathrm{C}\right.$-ssNMR $)$ is another technique used to confirm the presence of a bonded moiety on the product silica hydride surface. NMR analysis of solids for dilute nuclei such as ${ }^{13} \mathrm{C}$ typically faces two 
challenges: the low spectra resolution associated with chemical shift anisotropy (CSA) and heteronuclear dipolar coupling (DC), and low sensitivity due to rarity and the low gyromagnetic ratio of ${ }^{13} \mathrm{C}$ nuclei.

In typical liquid-state applications of NMR, the resulting spectrum consists of sharp peaks, due to averaging of chemical shift anisotropic effect to zero by Brownian motion (random, rapid reorientation of nuclei) in the sample solution. However, the random movement of sample is restricted in solids, thereby analyte molecules result in an immobilized state at random orientation with respect to the magnetic field. Since chemical shift is also orientation-dependent, the resulting chemical shift is an anisotropic broad peak. The CSA and DC effect can be reduced, if not neglected, by utilizing the magic angle spinning (MAS) technique. The magic angle $\left(54.7^{\circ}\right)$ is derived from solving the factor $3 \cos ^{2} \theta-1$, also known as the second-order Legendre polynomial, which is a small segment of Hamiltonian expression of the DC effect. MAS also reduces the CSA effect by mimicking the motion of fluid. This is done by introducing rapid $(1-25 \mathrm{kHz})$ artificial spin to a sample rotor at the magic angle with respect to applied magnetic field. Therefore, peak broadening effects from both CSA and DC are diminished on the NMR timescale resulting in an isotropic chemical shift analogous to solution-state NMR [72, 73].

The signal sensitivity issue for ${ }^{13} \mathrm{C}$ nuclei can be resolved with the cross polarization (CP) technique. The $\mathrm{CP}$ technique involves polarization energy transfer from abundant ${ }^{1} \mathrm{H}$ nuclei to rare ${ }^{13} \mathrm{C}$ nuclei by taking advantage of the heteronuclear dipolar coupling effect. In order to achieve energy transfer between the two nuclei with different 
gyromagnetic ratios $\left(\gamma_{\mathrm{P}}\right.$ and $\left.\gamma_{\mathrm{C}}\right)$, field intensity for protons $\left(\mathrm{H}_{1 \mathrm{P}}\right)$ and carbons $\left(\mathrm{H}_{1 \mathrm{C}}\right)$ must be adjusted to fulfill Hartmann-Hahn condition $\left(\gamma_{\mathrm{H}} \bullet \mathrm{H}_{1 \mathrm{P}}=\gamma_{\mathrm{C}} \bullet \mathrm{H}_{1 \mathrm{C}}\right)$. In this condition, precession frequencies of two nuclei match and spins in close vicinity will have strong heteronuclear dipolar coupling; thus, polarization energy can be resonance transferred [74]. The heteronuclear DC effect used for the energy transfer is decoupled by turning off only the carbon transmitter, and therefore the free inductive decay of ${ }^{13} \mathrm{C}$ nuclei is directly recorded to generate the NMR spectrum [75]. Overall, the CP technique allows for the signal enhancement of dilute nuclei.

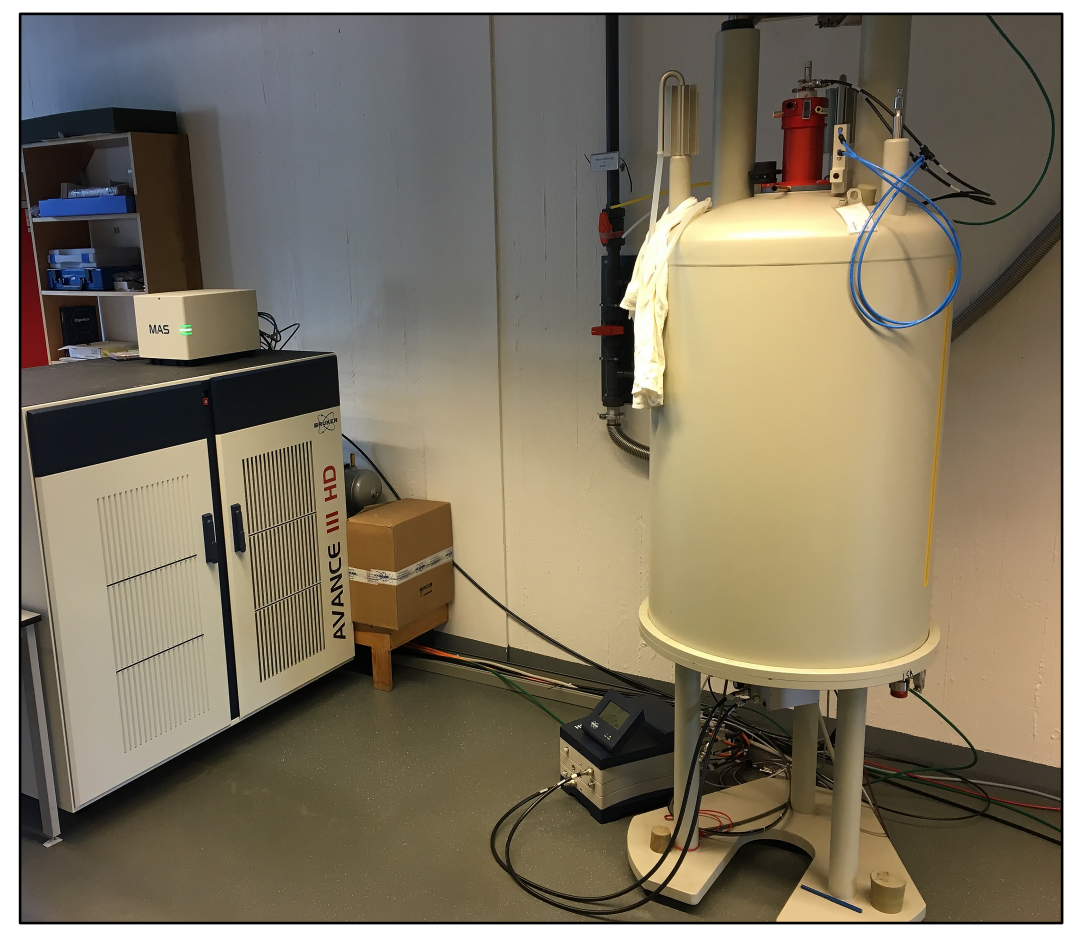

Figure 42. Instrument Used for ${ }^{13} \mathrm{C}$ CP-MAS Solid-state NMR Analysis.

The ${ }^{13} \mathrm{C}$ CP-MAS ssNMR spectra were collected by Dr. Markus Kramer at the University of Tübingen in Germany with a Bruker Avance III HD 300 WB NMR spectrometer equipped with a $4 \mathrm{~mm}$ CP-MAS Probe Head (Figure 42). The sample rotor was spun at $10 \mathrm{kHz}$. 


\subsubsection{HPLC - Diode Array Detector (DAD)}

About half of chromatographic studies utilize HPLC coupled with photo diode array detector (PDA or DAD), most particularly for column performance evaluation. HPLC with UV/DAD has much less solvent and buffer restrictions compared to LC/MS instrumentation; therefore, it allows for more efficient analysis of molecules sensitive to $\mathrm{pH}$ and/or ionic strength. DAD used in this study has multiple advantages over traditional UV detectors. While UV detectors employ single-wavelength scan, DADs can utilize multiple ranges of wavelengths and captures the whole UV/Vis and partial-NIR spectrum. For instance, the Agilent 1200 series HPLC system utilized in this study has a detection range of $190 \mathrm{~nm}$ up to $950 \mathrm{~nm}$ (Figure 43). The peak area of analyte absorbance can be utilized for quantitative measurement, and whole UV/Vis spectra of each peak allows for presumptive identification of analytes (Figure 44). This feature is particularly useful in the food/pharmaceutical industries which allows them to assess the spectral homogeneity of an analyte peak, also known as "peak purity" [76]. In addition to chromatographic purity, peak purity assessment ensures the absence of a co-eluting species in the analyte peak of interest by evaluating the diode array spectra at different points (e.g., front, apex, back) in a single peak. 


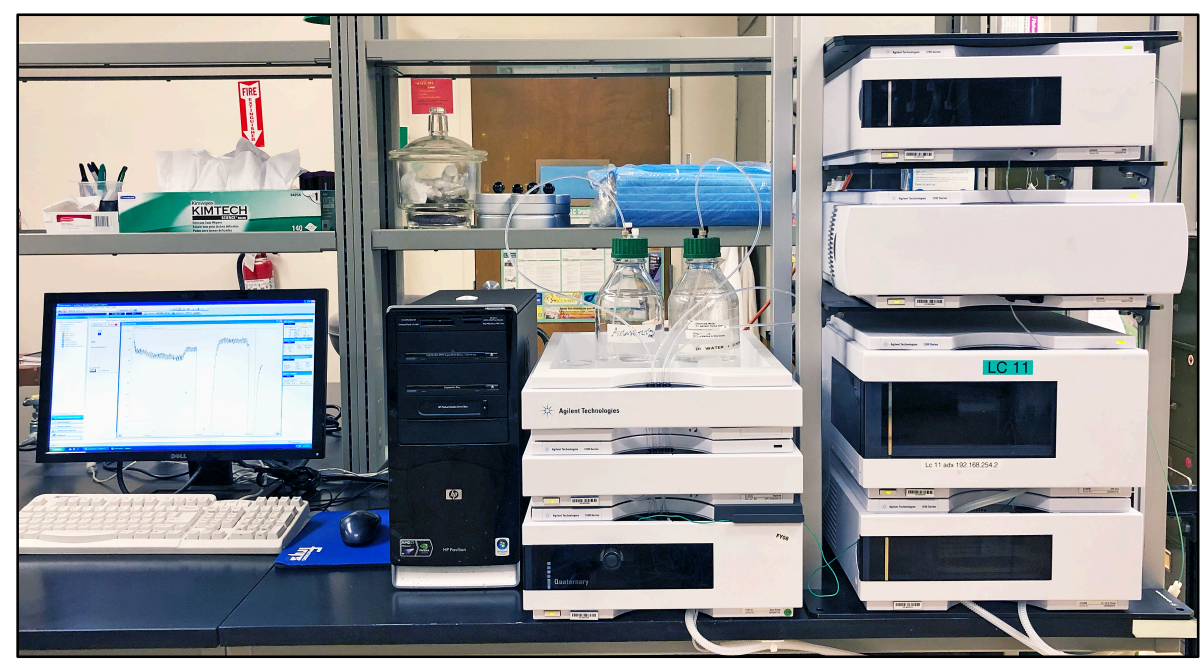

Figure 43. Agilent 1200 Series HPLC Instrument.

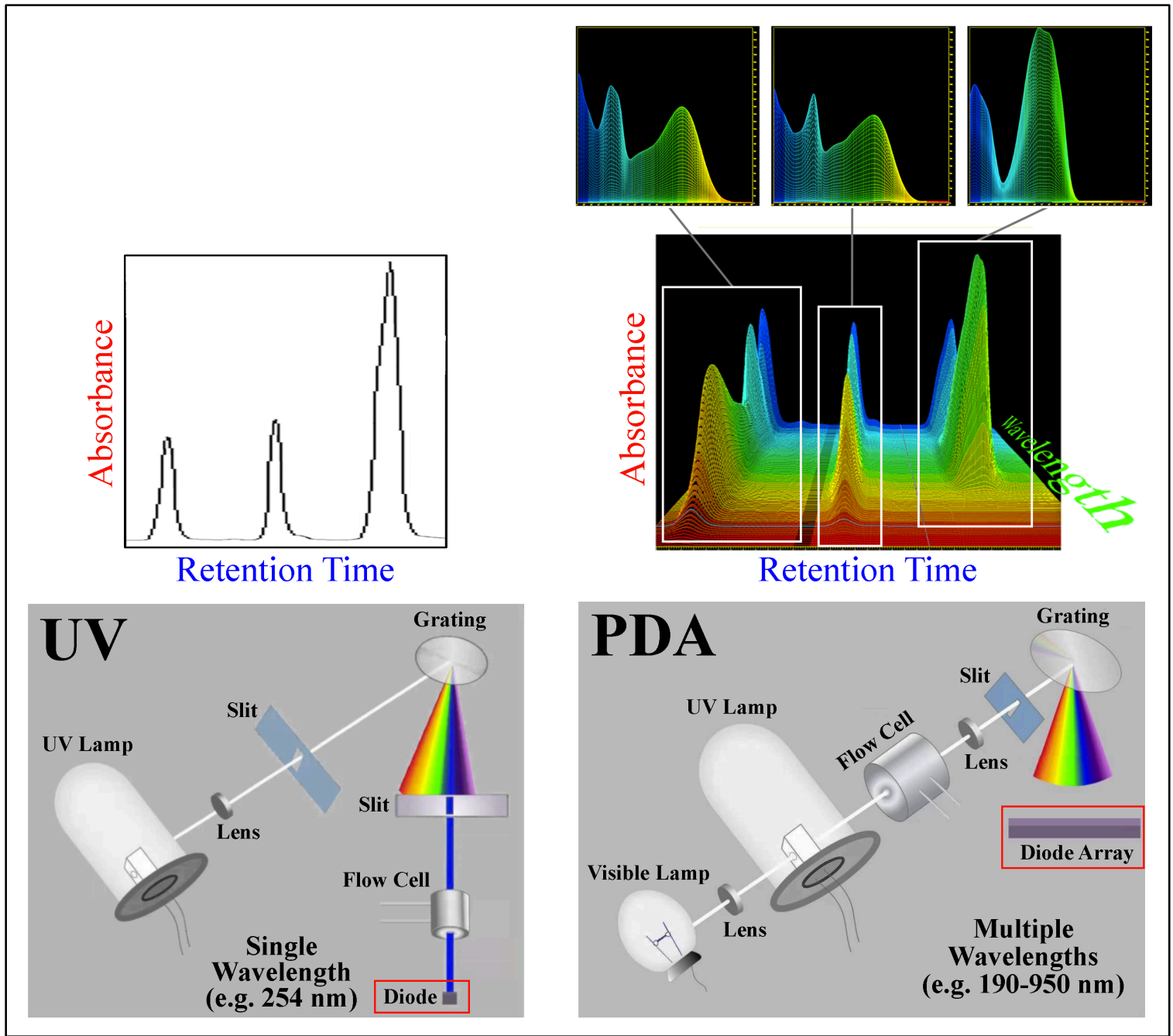

Figure 44. Scheme for UV and PDA Detector. 


\subsubsection{LC/MS - Electrospray Ionization Time-of-flight (ESI-TOF-MS)}

Two limitations of HPLC-UV are: (1) the analyte of interest must have chromophore, and (2) difficulties of separation and data interpretation upon analysis of a complex sample mixture. LC/MS is a powerful alternative that avoids time-consuming analyte derivatization steps and requires significantly less chromatographic method optimization. Therefore, LC/MS was utilized for the analysis of complex biological samples such as extracts of cannabis and grape skin, and UV-inactive carbohydrates. The LC/MS instrument used in this study was an Agilent 1100/1200 Series HPLC coupled to a 6200 Series Dual Electrospray Ionization Orthogonal-acceleration Time-of-Flight Mass Analyzer (ESI-TOF; Figure 45).

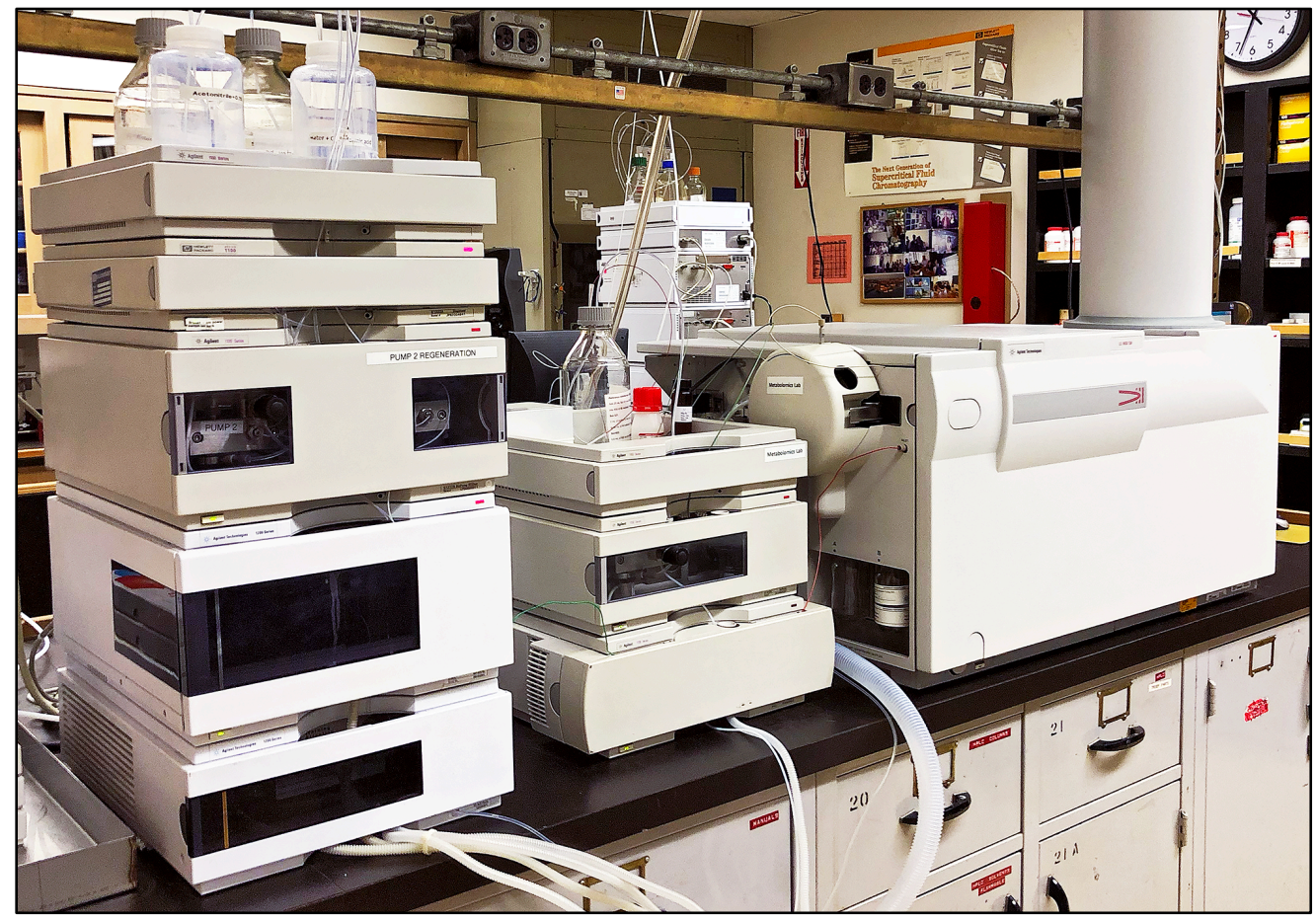

Figure 45. Agilent 1100/1200 Series HPLC Coupled with 6200 Series ESI-TOF-MS.

The first component of the mass spectrometer is an ionizer called ESI, which is illustrated in (Figure 46). 


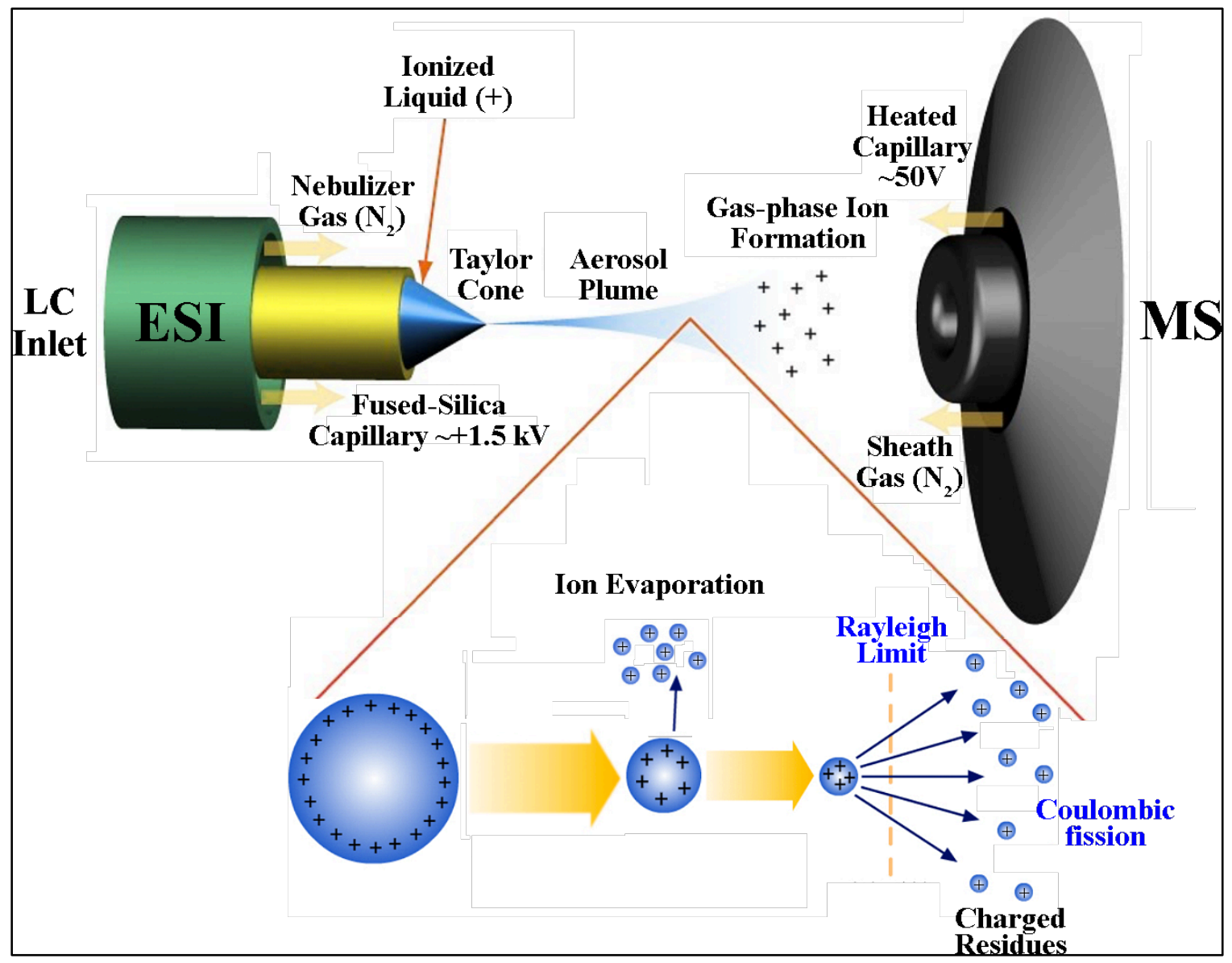

Figure 46. Scheme for Electrospray Ionization.

Upon sample entry from the LC system, the electrospray moves the liquid containing the analyte(s) of interest through a capillary nozzle and disperses into a vacuum as a fine aerosol. The droplets are charged and decreased in size as the solvent evaporates. The charge density of the droplet increases until it reaches the Rayleigh limit, where the electrostatic repulsion of charges overcomes the surface tension of the droplet and causes it to explode (Coulomb fission). This process forms smaller charged-droplets and repeats until complete evaporation and leaves behind gas-phase analyte ions [64]. Same analyte can be charged in multiple ways and produces several ions known as "adducts" (e.g., $[\mathrm{M}+\mathrm{H}]^{+}$and $\left[\mathrm{M}+\mathrm{NH}_{4}\right]^{+}$) which depends on the mobile phase and/or sample composition. 
The TOF analyzer is the second major component in the LC/MS instrument and it is commonly coupled with an electrospray ionizer. Mass analyzer facilitates "extraction" or essentially distinguishes analyte ions based on their $\mathrm{m} / \mathrm{z}$ (mass-to-charge) ratio. Mass extraction is achieved by applying an electric field of known strength which gives ions the same amount of kinetic energy. However, the velocity of ions and the "time-offlight" from the starting point to the detector varies, and it is dependent on the $\mathrm{m} / \mathrm{z}$ ratio. For traditional TOF instruments, mass resolution is limited due to kinetic energy distribution of ions upon its entry into the analyzer. Modern TOF instruments correct this fluctuation by utilizing a reflectron, and by orthogonally accelerating the analyte ions.

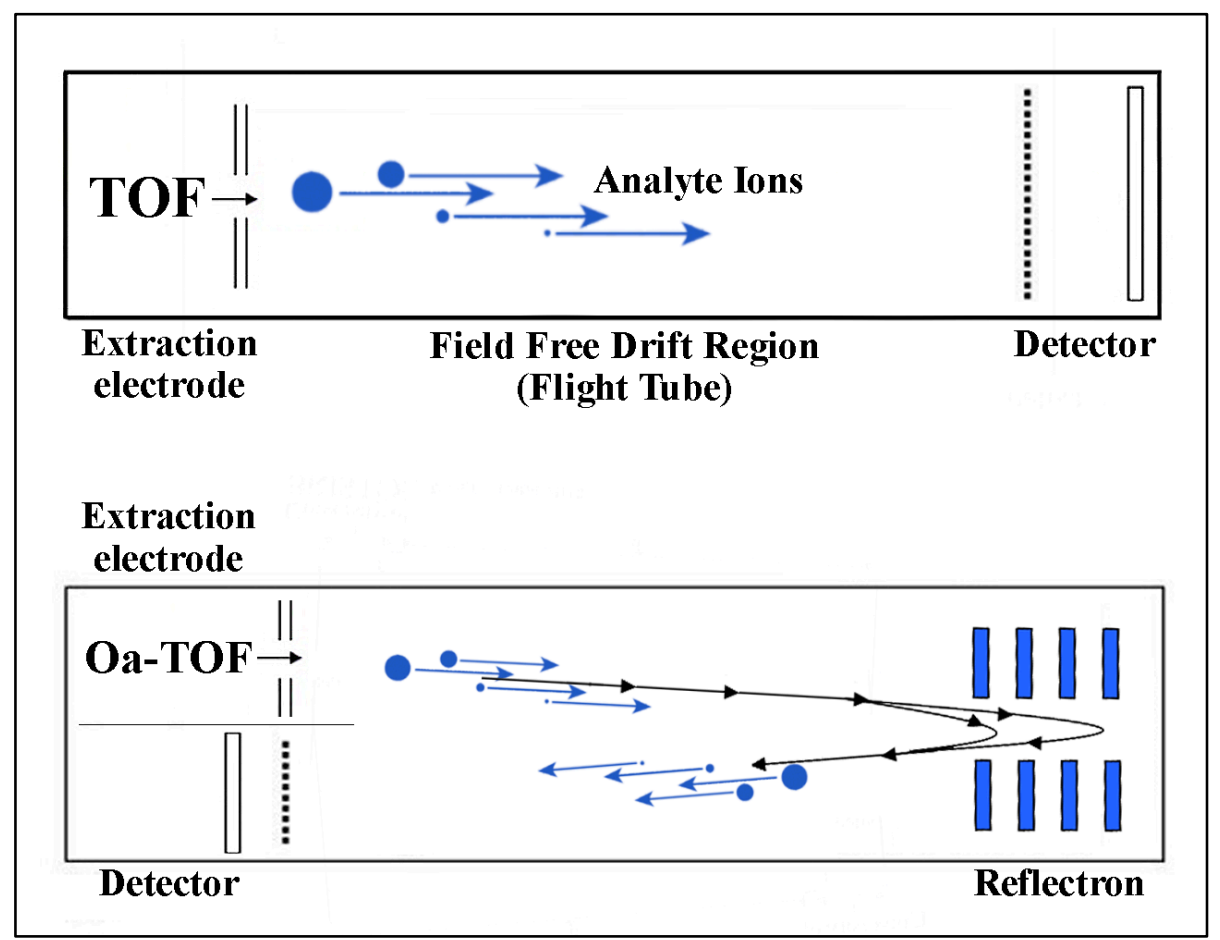

Figure 47. Scheme for Traditional (Top) and Orthogonal-acceleration TOF (Below).

As shown in Figure 47, an ion with higher kinetic energy penetrates deeper into the reflectron compared to the same $\mathrm{m} / \mathrm{z}$ ion with lower energy; thereby adjusting the flight path length of ions according to their differences in kinetic energy [77]. One 
advantageous feature of TOF is that there is no theoretical limit to $\mathrm{m} / \mathrm{z}$ values that the analyzer can detect. Thus, ESI-TOF can analyze a wide range of ionizable large molecules such as proteins.

\section{RESULTS AND DISCUSSION}

\subsection{Simulations of the Hydride Surface}

MD simulations of ordinary silica and silica hydride surfaces were performed to visually understand their behavior differences in aqueous media. Figure 48 was generated using MMFF94 energy minimization and simulated at $298 \mathrm{~K}$ in a $100 \%$ aqueous environment.

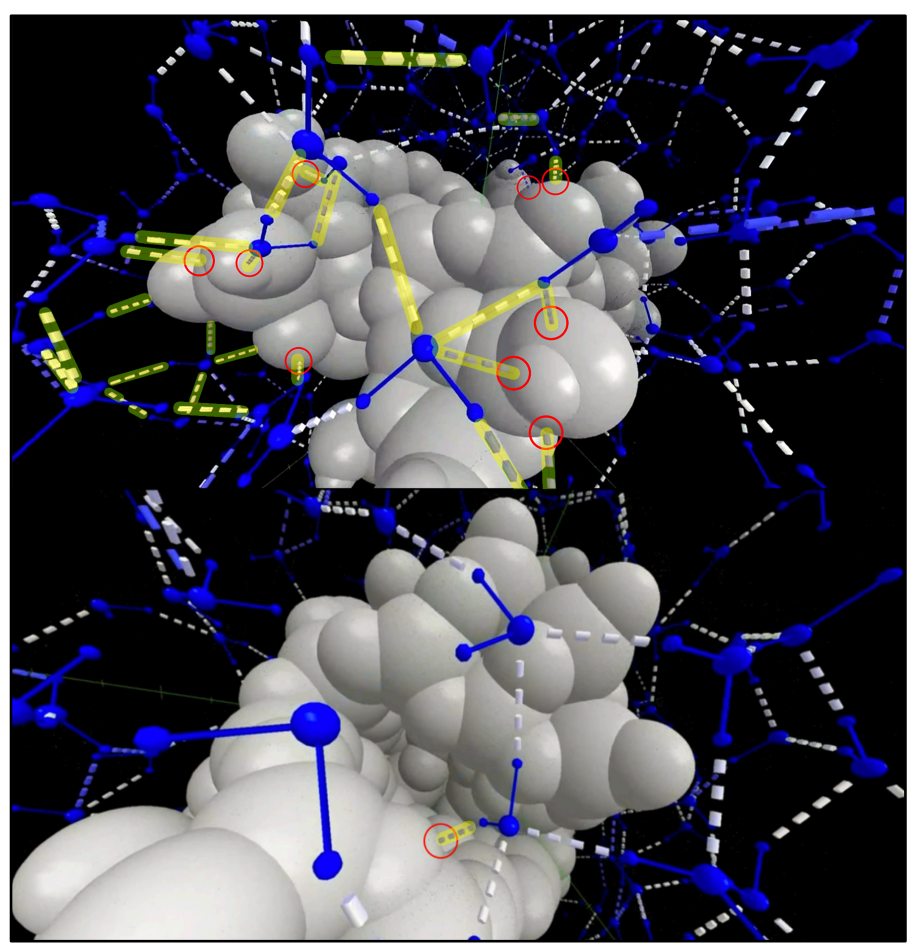

Figure 48. MD Simulations of Ordinary (Top) and Hydride (Bottom) Silica Surfaces.

Ordinary silica (top) actively interacts with surrounding water molecules by hydrogen bonding (circled in red) and forms an immobilized hydration layer (as highlighted in 
yellow). This behavior resembles that of chaotropic agents described in the Hofmeister ion interaction theory, which increases the entropy of the solvent system by engaging with and disrupting the H-bonding networks of water molecules. In contrast, silica hydride materials (bottom) have minimal interactions with surrounding water molecules and do not immobilize bulk water around its surface. Other than virtually non-existent silanols, siloxane ( $\mathrm{Si}-\mathrm{O}-\mathrm{Si}$ ) is the only functional group that participates in weak $\mathrm{H}$ bonding interaction with water [78].

\subsection{Hydrosilylation Products}

The weight of reaction products is shown in Table 14. All bonded phases except for LEU are pictured in Figure 49. Only the TOCO phase had a characteristic light brown color and the rest were uncolored.

Table 14. Summary of Hydrosilylation for the Four Phases.

\begin{tabular}{|l|c|c|c|c|}
\hline & TOCO & PFP & C20 & LEU \\
\hline Wt. of silica used (grams) & 5.0076 & 5.0016 & 5.0030 & 2.6634 \\
\hline Wt. of olefin used (grams) & 17.0400 & 8.3525 & 11.2038 & 6.9900 \\
\hline Wt. of product (grams) & 5.2206 & 5.0900 & 5.4280 & 2.8754 \\
\hline
\end{tabular}

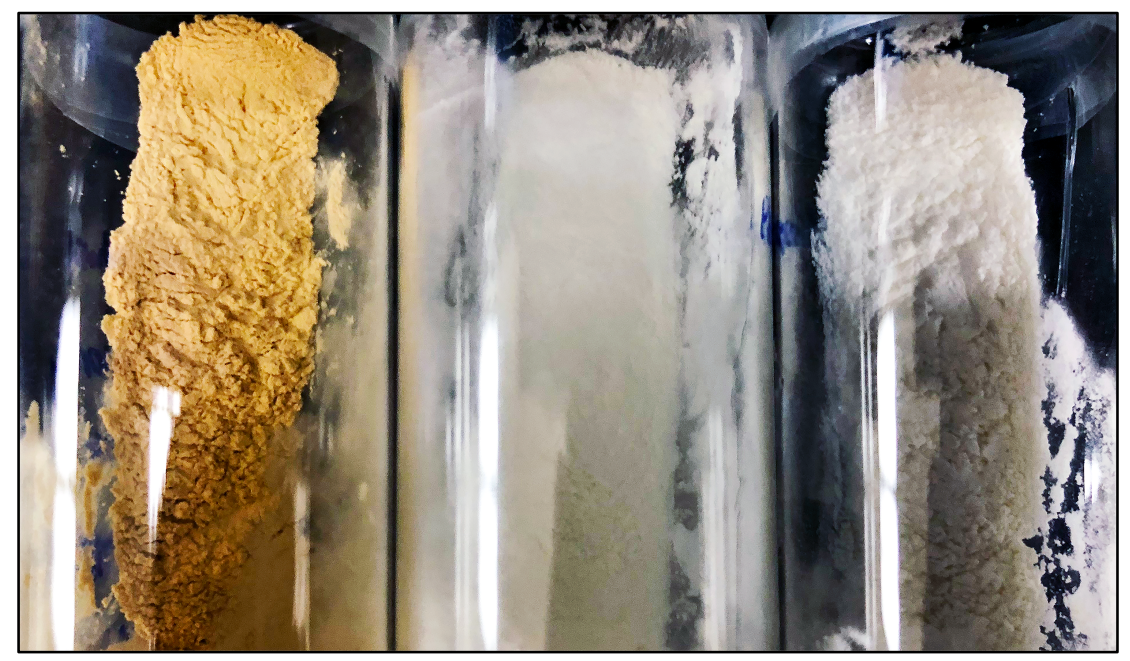

Figure 49. The Reacted Bonded Phases. (From left: TOCO, PFP, C20). 


\subsection{ATR/DRIFTS Characterization}

The reaction products were first characterized by IR spectroscopic techniques. Prior to the investigation of actual bonded phases, ATR spectra of the starting olefin materials were collected as references (Figure 50-53). The successful hydrosilylation reaction can be confirmed when the same characteristic ATR absorbance peaks are observed in the DRIFT spectra of the products. For instance, C20 has two sharp peaks at 2850 and 2920 $\mathrm{cm}^{-1}$ region corresponding to $\mathrm{C}-\mathrm{H}$ stretch (Figure 54). PFP has the aromatic $\mathrm{C}=\mathrm{C}$ stretch peak at $1500 \mathrm{~cm}^{-1}$ region and C-F stretch at $1100 \mathrm{~cm}^{-1}$ region (Figure 55). For TOCO, characteristic $\mathrm{C}-\mathrm{H}$ stretch was observed at $2900 \mathrm{~cm}^{-1}$, aromatic $\mathrm{C}=\mathrm{C}$ stretch at about 1450 $\mathrm{cm}^{-1}$, and C-O stretch around $1080 \mathrm{~cm}^{-1}$ (Figure 56). Finally, LEU has C-H stretch at $2900 \mathrm{~cm}^{-1}$, C-O stretch at $1227 \mathrm{~cm}^{-1}$, and an intense peak at $1716 \mathrm{~cm}^{-1}$ region from overlap of $\mathrm{C}=\mathrm{O}$ stretch, $\mathrm{C}=\mathrm{C}$ stretch, and $\mathrm{N}-\mathrm{H}$ bend (Figure 57). Reacted products are also expected to show depletion in $\mathrm{Si}-\mathrm{H}$ stretch at $2250 \mathrm{~cm}^{-1}$ due to replaced hydride group by the organic moieties, and $\mathrm{C}=\mathrm{C}$ stretch at $1630-1650 \mathrm{~cm}^{-1}$ region from the loss of terminal alkene group of the reactant olefins. In summary, all four DRIFT spectra of silica hydride bonded phases contain the above-mentioned characteristic peaks from the ATR spectra of the olefins. 


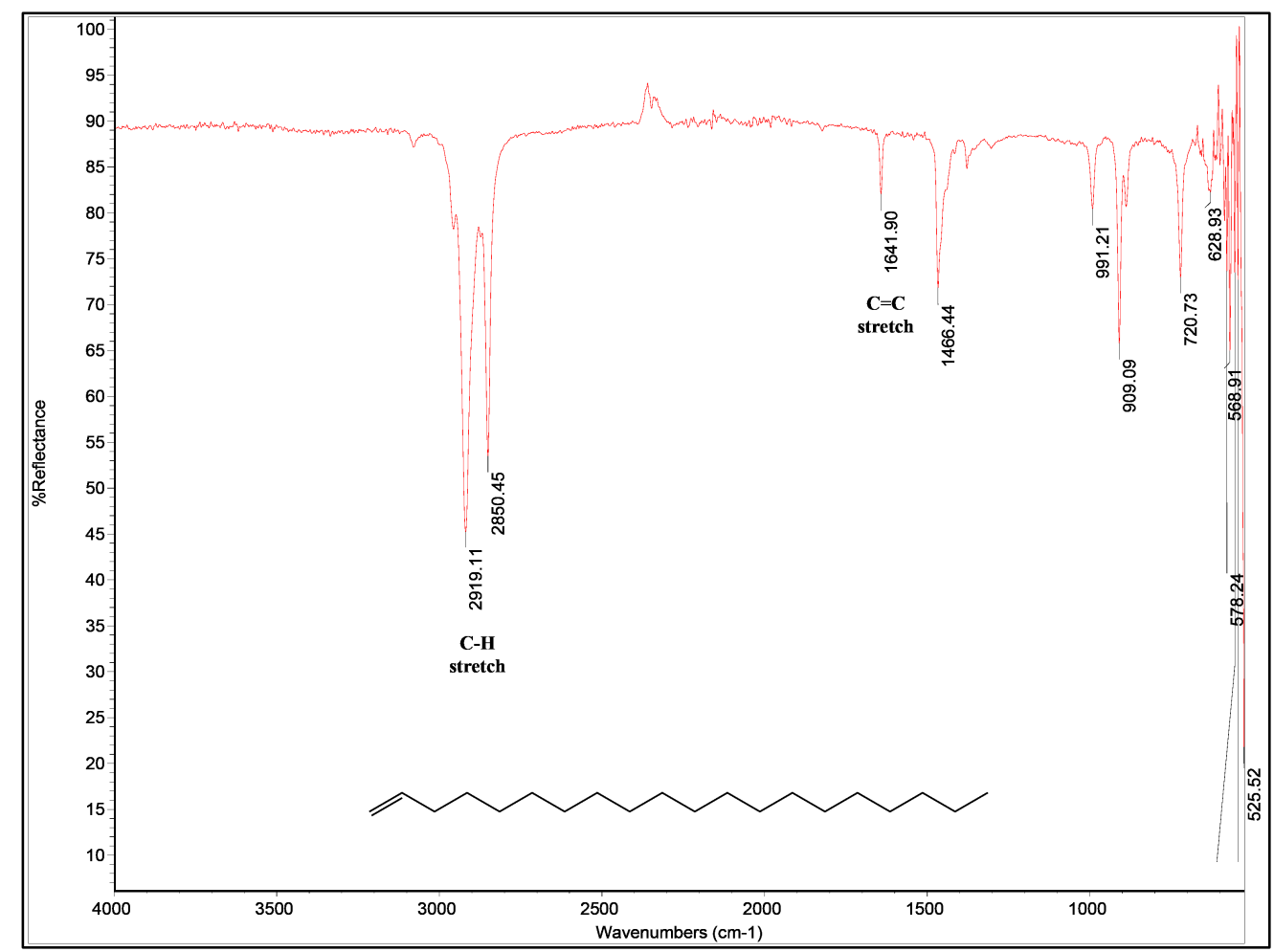

Figure 50. ATR Spectra of 1-eicosene (C20).

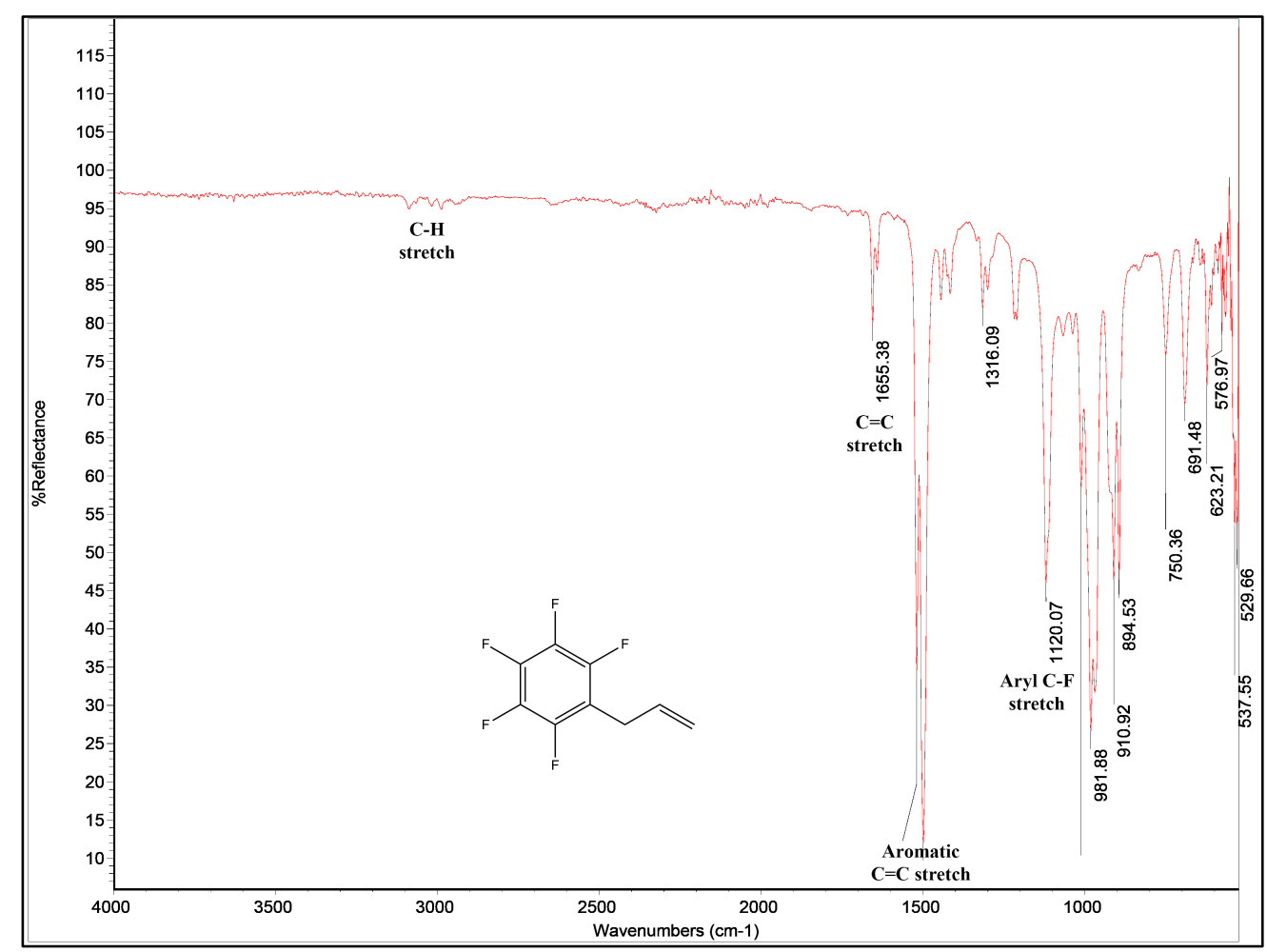

Figure 51. ATR Spectra of Allylpentafluorobenzene (PFP). 


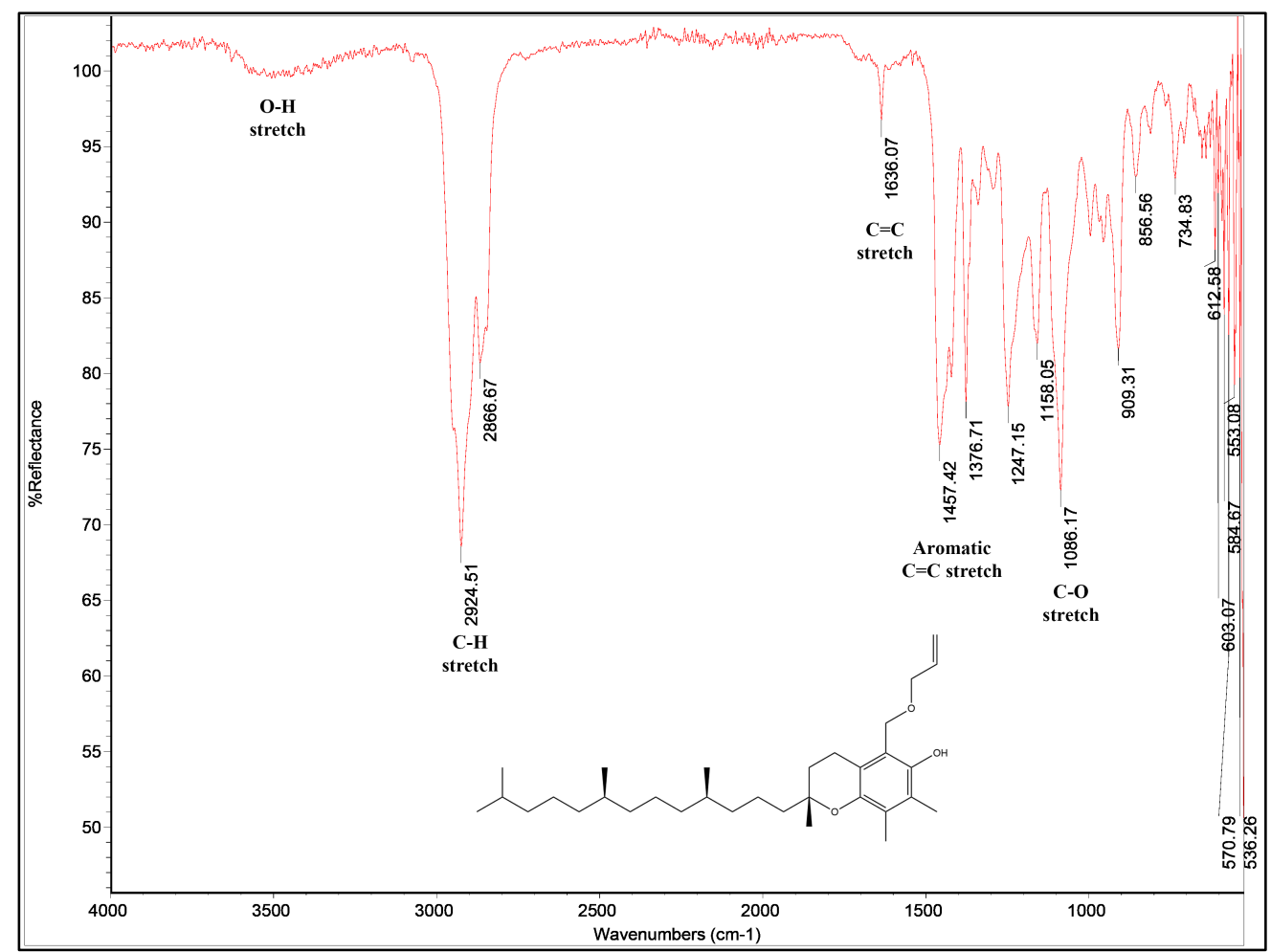

Figure 52. ATR Spectra of Allyloxy-DL-alpha-tocopherol (TOCO).

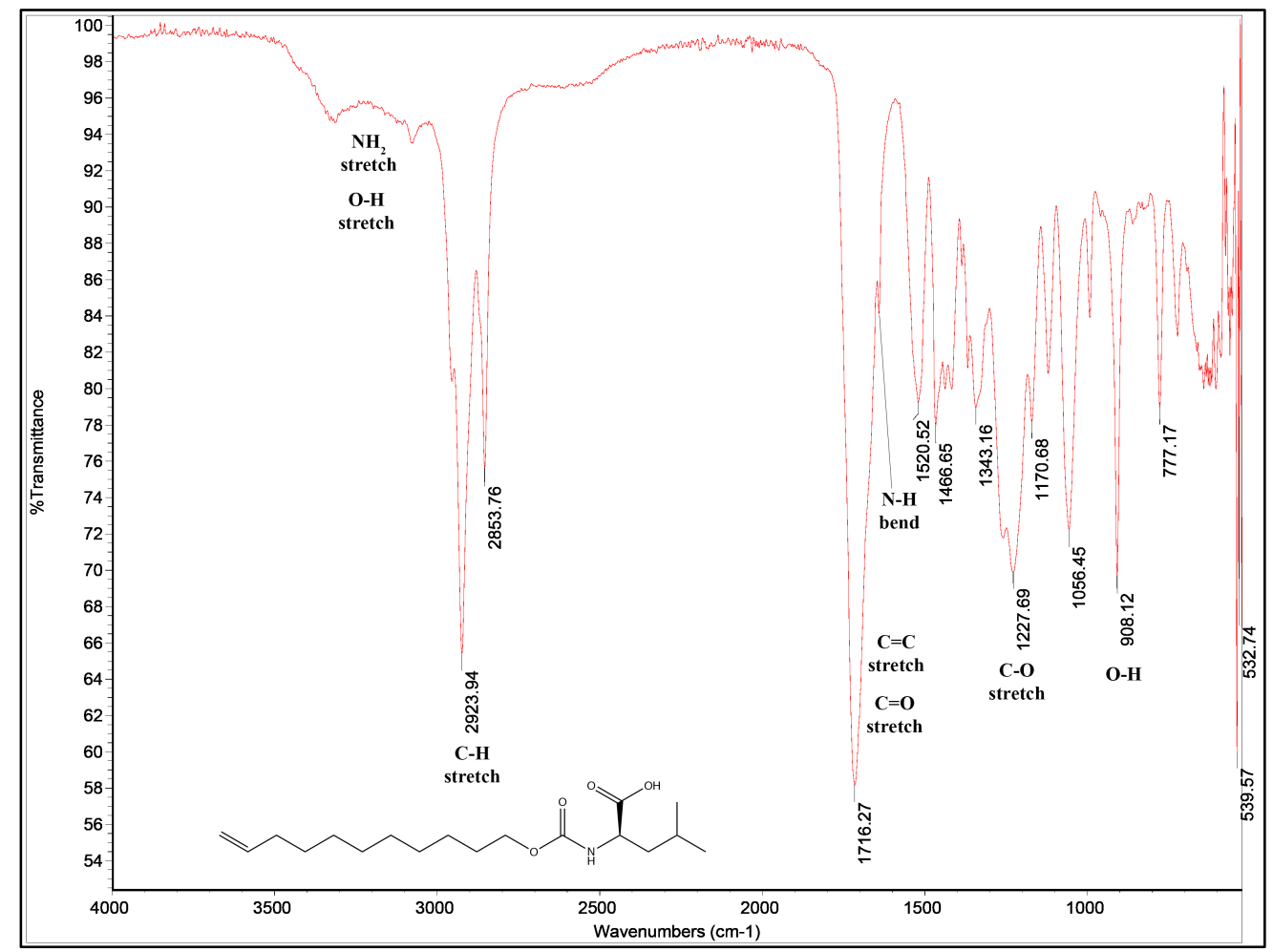

Figure 53. ATR Spectra of 10-undecenyloxycarbonyl-leucine (LEU). 


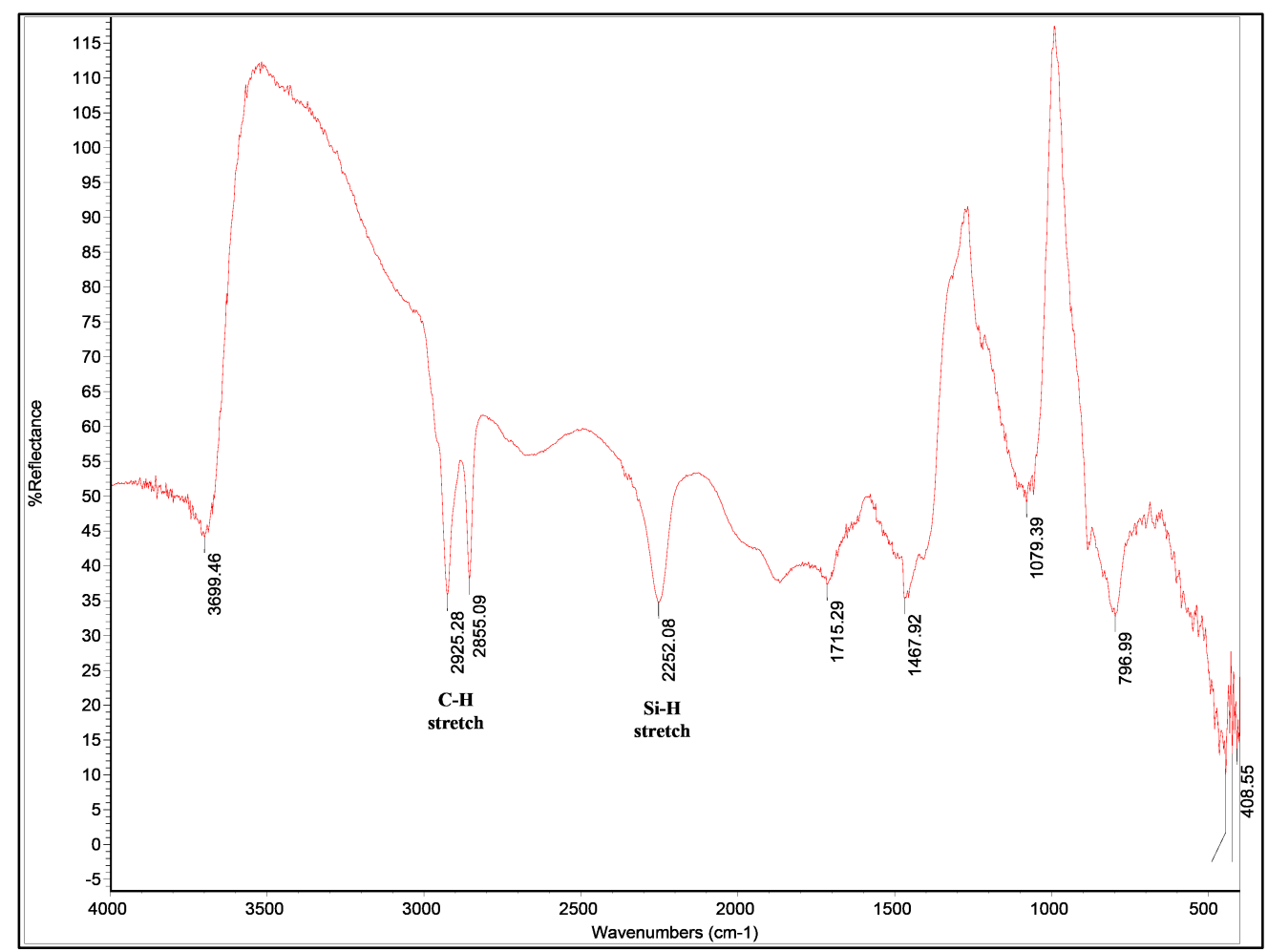

Figure 54. DRIFT Spectra of C20 Hydride.

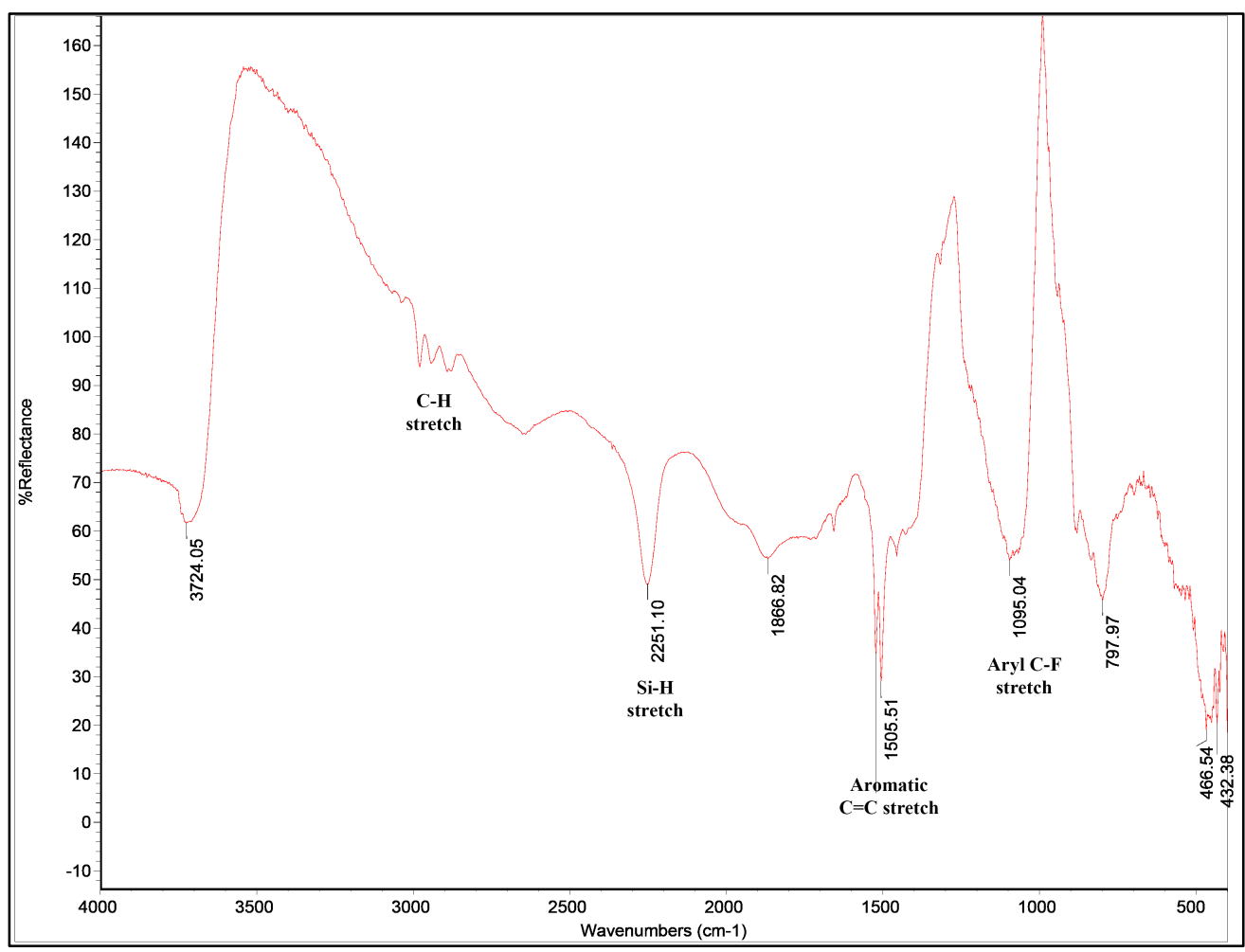

Figure 55. DRIFT Spectra of PFP Hydride. 


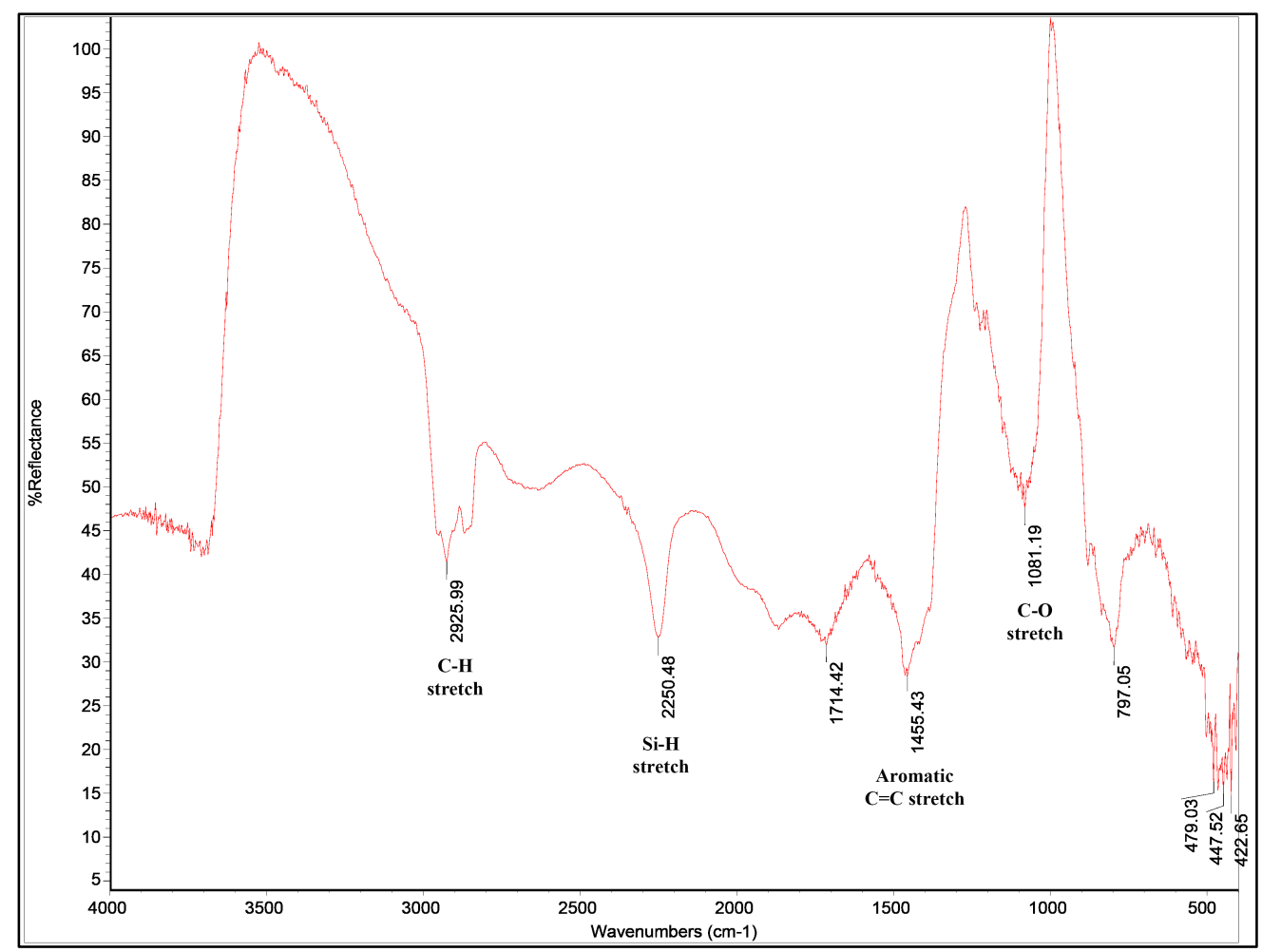

Figure 56. DRIFT Spectra of TOCO Hydride.

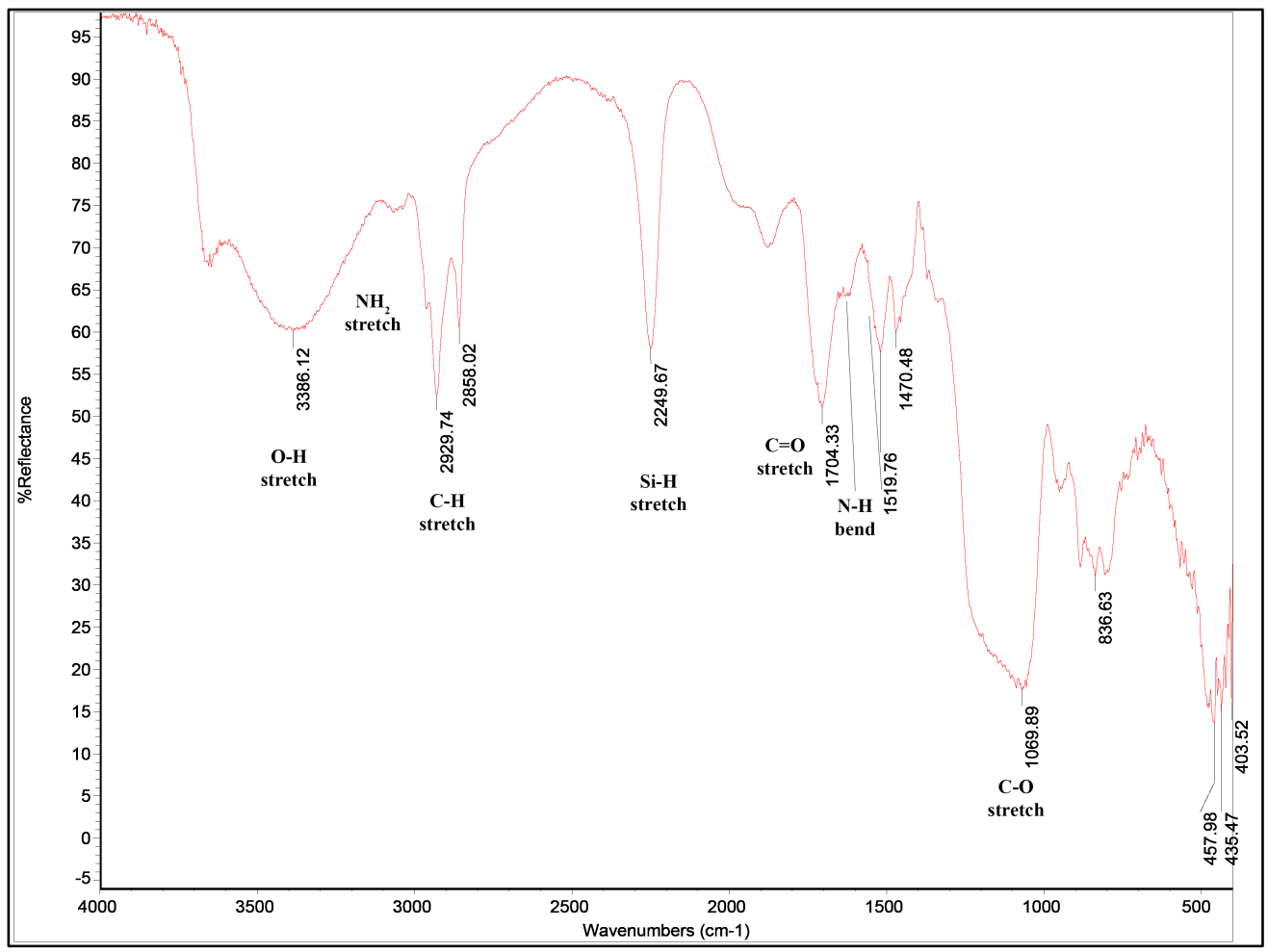

Figure 57. DRIFT Spectra of LEU Hydride. 
Furthermore, high-temperature DRIFT spectroscopy was utilized to study the water desorption profile of ordinary silica and silica hydride materials. Although silica hydride does not strongly adsorb water at its surface like regular silica, the previous DSC study suggests the presence of tightly bound water molecules that stays within the silica hydride lattice until $400-450^{\circ} \mathrm{C}$ [79]. To better comprehend the nature of hydride materials, variable-temperature DRIFTS (VT-DRIFTS) experiments were conducted in collaboration with Professor Abraham Wolcott (San Jose State University). Both silica samples (in $\mathrm{KBr}$ bulk matrix) were heated in the reaction chamber (Figure 58) until the majority of O-H stretch at $3000-3500 \mathrm{~cm}^{-1}$ has diminished.

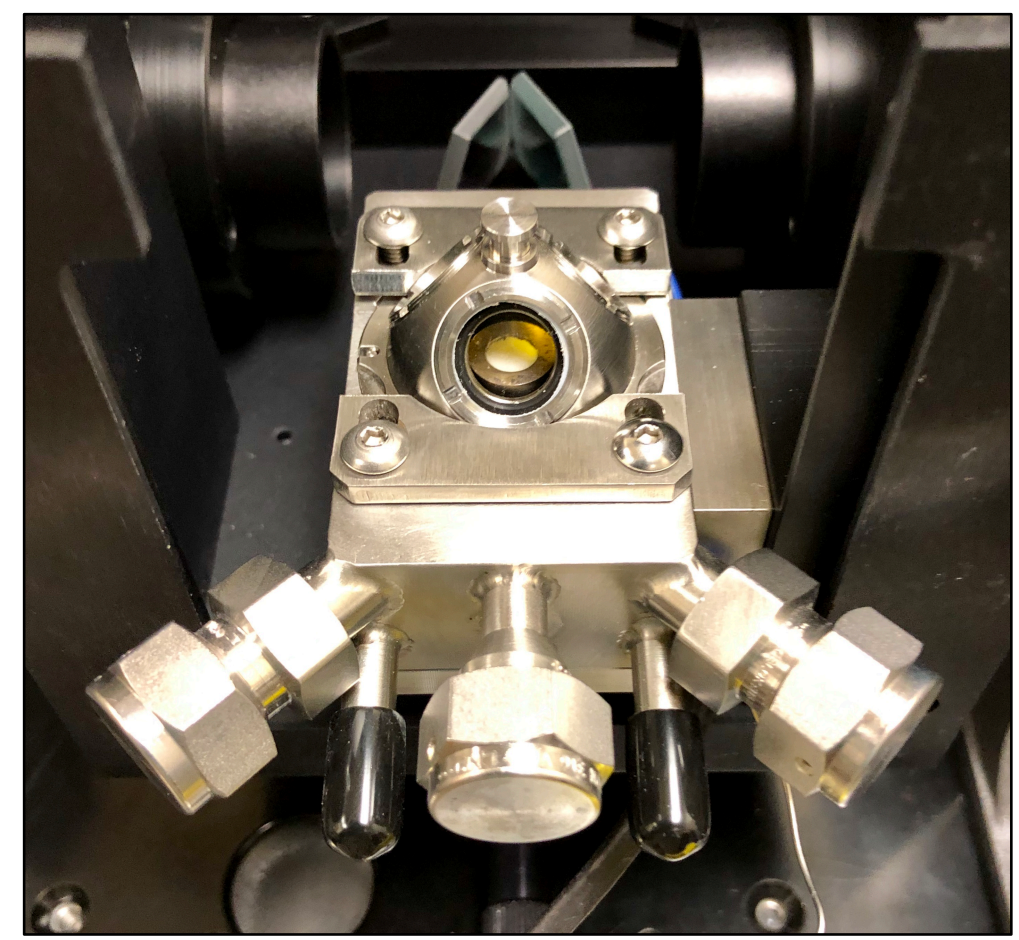

Figure 58. Variable-temperature DRIFTS Chamber.

Figure 59 shows the overlaid DRIFT spectra of the two silica materials at room temperature. As a result, the $\mathrm{O}-\mathrm{H}$ stretch absorbance depletes at $100{ }^{\circ} \mathrm{C}$ for the ordinary silica material (Figure 60). 


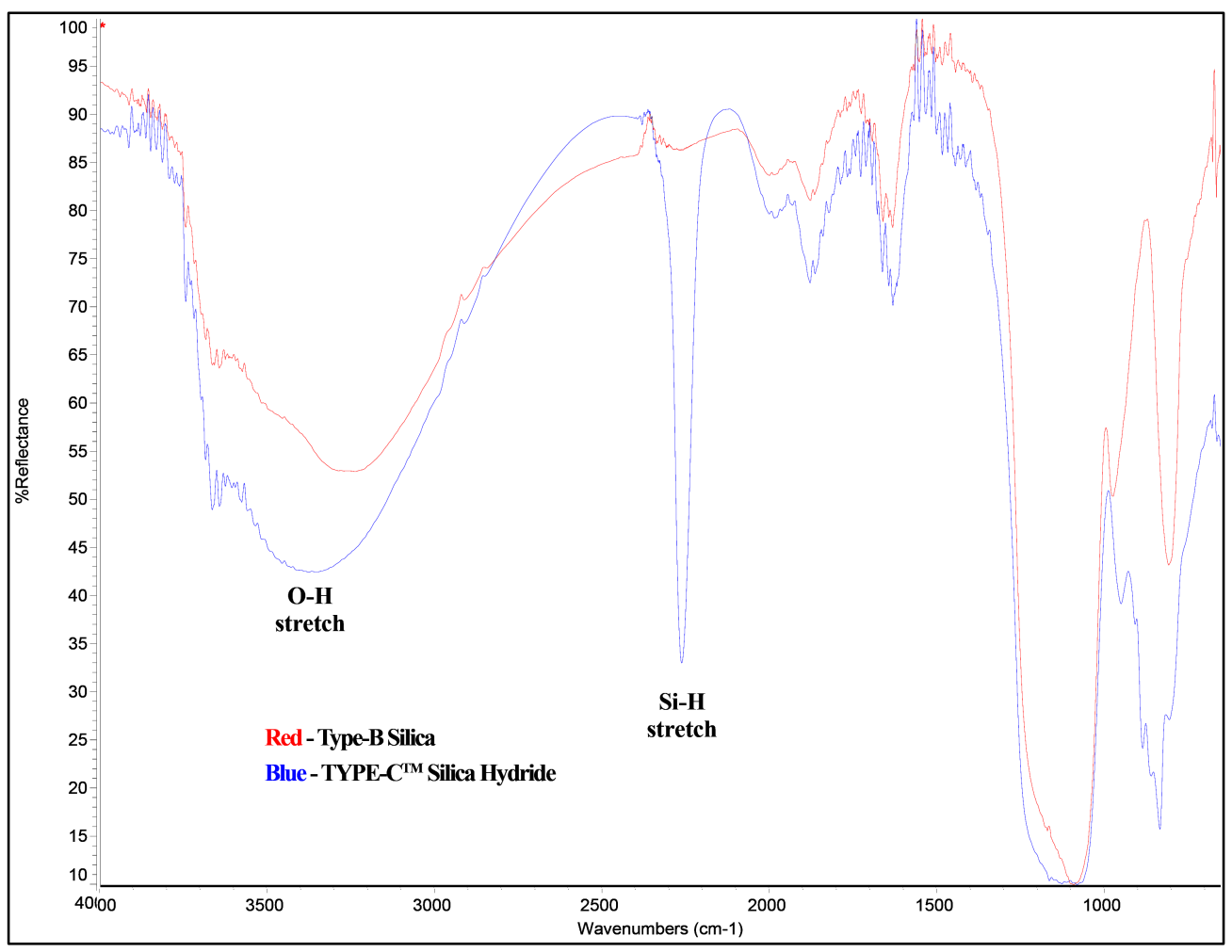

Figure 59. VT-DRIFT Spectra of Two Silica Materials at Room Temperature.

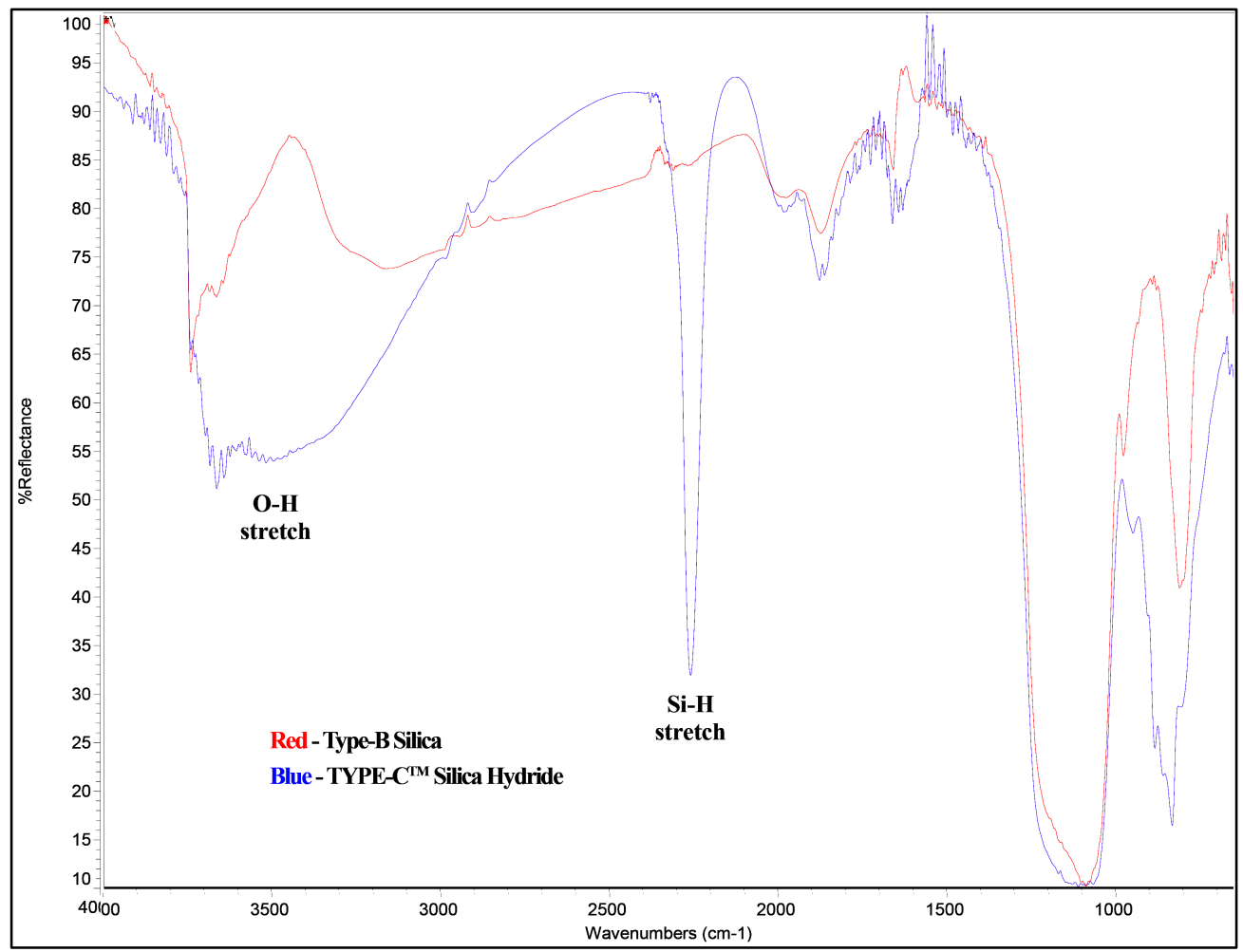

Figure 60. VT-DRIFT Spectra of the Two Silica Materials at $100{ }^{\circ} \mathrm{C}$. 
In the meantime, water desorption from the silica hydride material was not significant until $400-450{ }^{\circ} \mathrm{C}$ where Si-H starts to oxidize (Figure 61). Thus, the result shown here is consistent with the thermal analysis conducted in the previous studies [79].

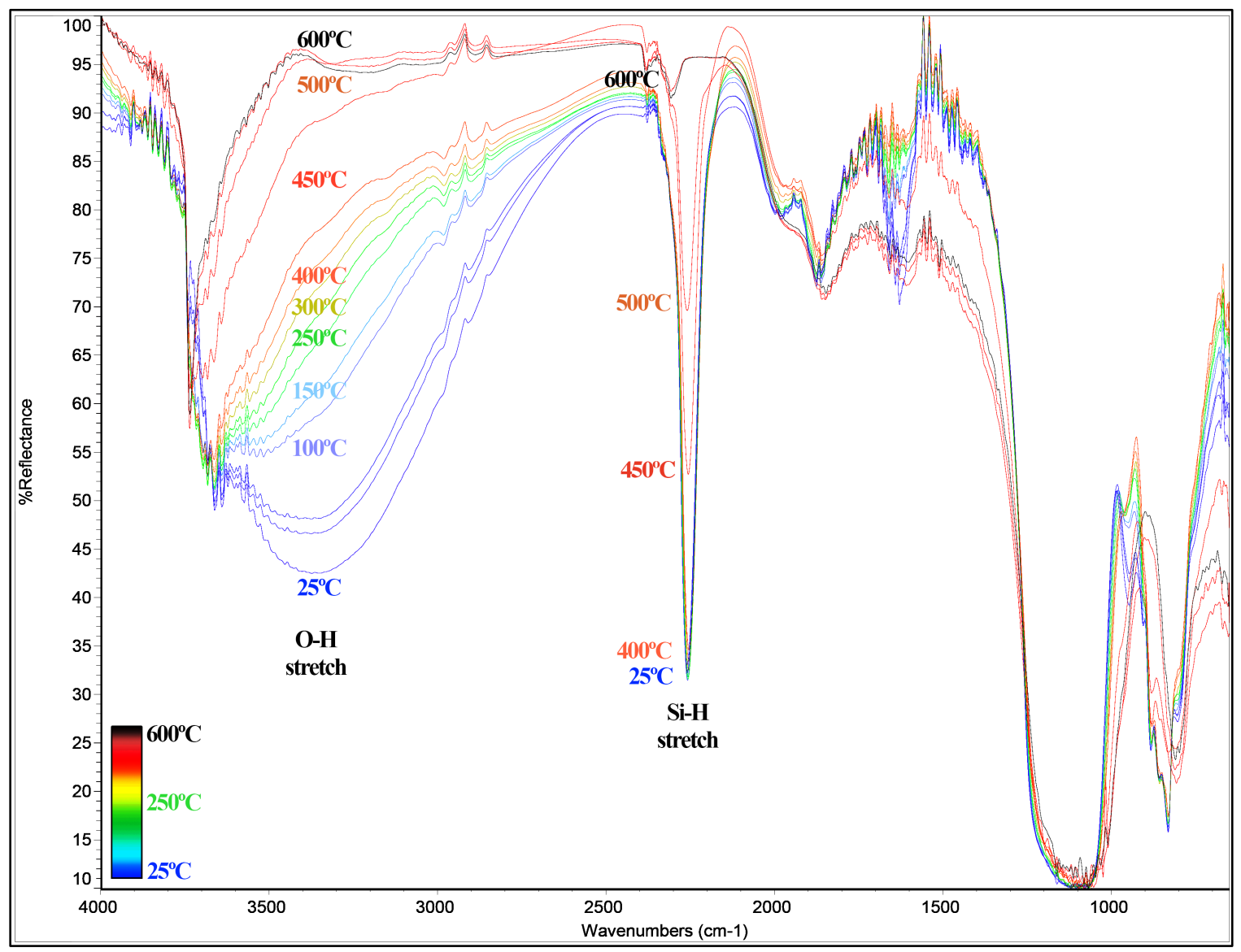

Figure 61. Overlaid VT-DRIFT Spectra of Silica Hydride from $25^{\circ} \mathrm{C}-600^{\circ} \mathrm{C}$.

\subsection{Elemental Carbon Analysis - Surface Coverage}

Bonded organic moieties were quantitatively evaluated by elemental carbon analysis. Surface coverage $\left(\mu \mathrm{mol} / \mathrm{m}^{2}\right)$ of the bonded phase was calculated by using the Berendsen - de Galan equation (Table 15). The extent of ligand coverage has numerous influences on chromatographic performance of the resulting columns. Generally, RP retention is enhanced by total carbon load and/or the hydrophobicity of bonded moiety. For high-end 
commercial C18 stationary phases, the surface coverage can be about $3.5 \mu \mathrm{mol} / \mathrm{m}^{2}$.

Lower coverage is known to increase the NP retention mode; furthermore, coverage of about $1.5 \mu \mathrm{mol} / \mathrm{m}^{2}$ is said to be enough for providing unique chromatographic features associated with the properties of bonded organic moieties [14].

Table 15. Carbon Content and Surface Coverage of the Bonded Moieties.

\begin{tabular}{|c|c|c|c|c|c|}
\hline Bonded Moiety & $\begin{array}{c}\text { Number } \\
\text { of } \\
\text { Carbons }\end{array}$ & $\begin{array}{c}\text { Molecular } \\
\text { Weight } \\
\text { (g/mol) }\end{array}$ & $\begin{array}{c}\text { SBET }_{\text {BET }} \\
\left(\mathrm{m}^{2} / \mathrm{g}\right)\end{array}$ & $\% \mathrm{C}$ & $\begin{array}{c}\text { Surface } \\
\text { Coverage } \\
\left(\mu \mathrm{mol} / \mathrm{m}^{2}\right)\end{array}$ \\
\hline $\begin{array}{l}\text { Allyloxy-DL-alpha-tocopherol } \\
\text { (TOCO) }\end{array}$ & 32 & 486.781 & \multirow{4}{*}{400} & 15.64 & 1.269 \\
\hline $\begin{array}{l}\text { Allylpentafluorobenzene } \\
\text { (PFP) }\end{array}$ & 9 & 208.131 & & 6.56 & 1.736 \\
\hline $\begin{array}{l}\text { 1-eicosene } \\
(\mathrm{C} 20)\end{array}$ & 20 & 280.540 & & 11.87 & 1.434 \\
\hline $\begin{array}{c}\mathrm{N} \text {-undecenoxycarbonyl-leucinate } \\
\text { (LEU) }\end{array}$ & 18 & 327.470 & & 9.52 & 1.286 \\
\hline
\end{tabular}

\section{5 ${ }^{13}$ C CP-MAS Solid-state NMR}

The ${ }^{13} \mathrm{C}$ CP-MAS solid-state NMR spectra were collected as another way to determine the bonding of organic moieties. In cross-polarized ${ }^{13} \mathrm{C}$ solid-state NMR, only the carbons directly bonded to hydrogen atom(s) will provide intense signals. Aside from the CP effect, ssNMR spectra generally have low resolution and signal sensitivity. Therefore, the procedure was used only as a tentative determination rather than confirmatory. Spectra of the four novel phases are shown in Figure 62-65. All carbons directly bonded to hydrogens are indicated in red. The predicted chemical shift of carbons without protons are numbered in light gray color. It is also worth noting that 
some of the resonance peaks observed were not associated with the olefins. For instance, peaks for residual wash solvent can be observed around 60-80 ppm [80], as well as the alkene carbons from olefins at 110-150 ppm region [81]. According to Pesek et al. (2002), peaks present at 17 and $60 \mathrm{ppm}$ may correspond to the methyl and methylene carbons from residual ethoxy groups from the silanization process [82].

The spectra of C20-bonded silica hydride had consistent chemical shifts with the spectra prediction generated by Cambridge ChemDraw software. All carbons in 1eicosene are $\mathrm{sp}^{3}$ hybridized $\mathrm{C}-\mathrm{H}$, and therefore peaks are found in the 10-35 ppm region. The most upfield peak around $12 \mathrm{ppm}$ seen in every NMR spectrum can be assigned to the methylene group directly attached to the hydride silica surface (Si-C) [21], which essentially indicates successful bonding of organic moieties. Chemical shift peaks at 65 and $130 \mathrm{ppm}$ that were consistently observed across other bonded phases are possibly associated with residual solvent. Spectra of the PFP-bonded phase show intense peaks for the carbons directly bonded to protons at 11 and $22 \mathrm{ppm}$. Low-intensity downfield peaks between 100-150 ppm are consistent with the predicted chemical shifts for C-F aromatic carbons. For the TOCO-bonded phase, all C-H bonds were found between the 10-80 ppm region. The spectrum of the LEU-bonded phase consists of one broad peak between 0-50 ppm region. Peaks for carbon \#11 and \#13 were not observed due to significant background noise [69]. 


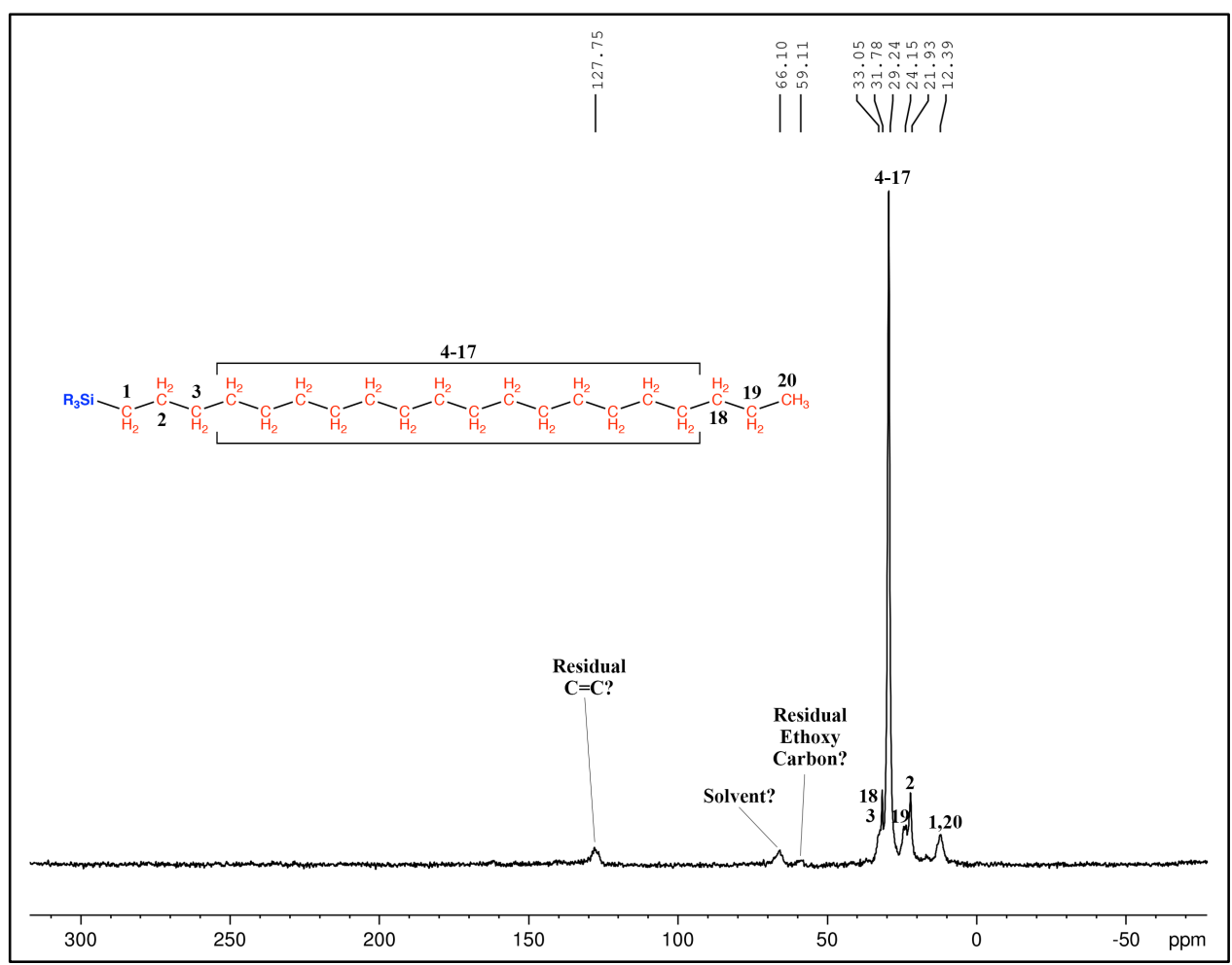

Figure 62. ${ }^{13} \mathrm{C}$ CP-MAS Solid-state NMR Spectra of C20 Hydride.

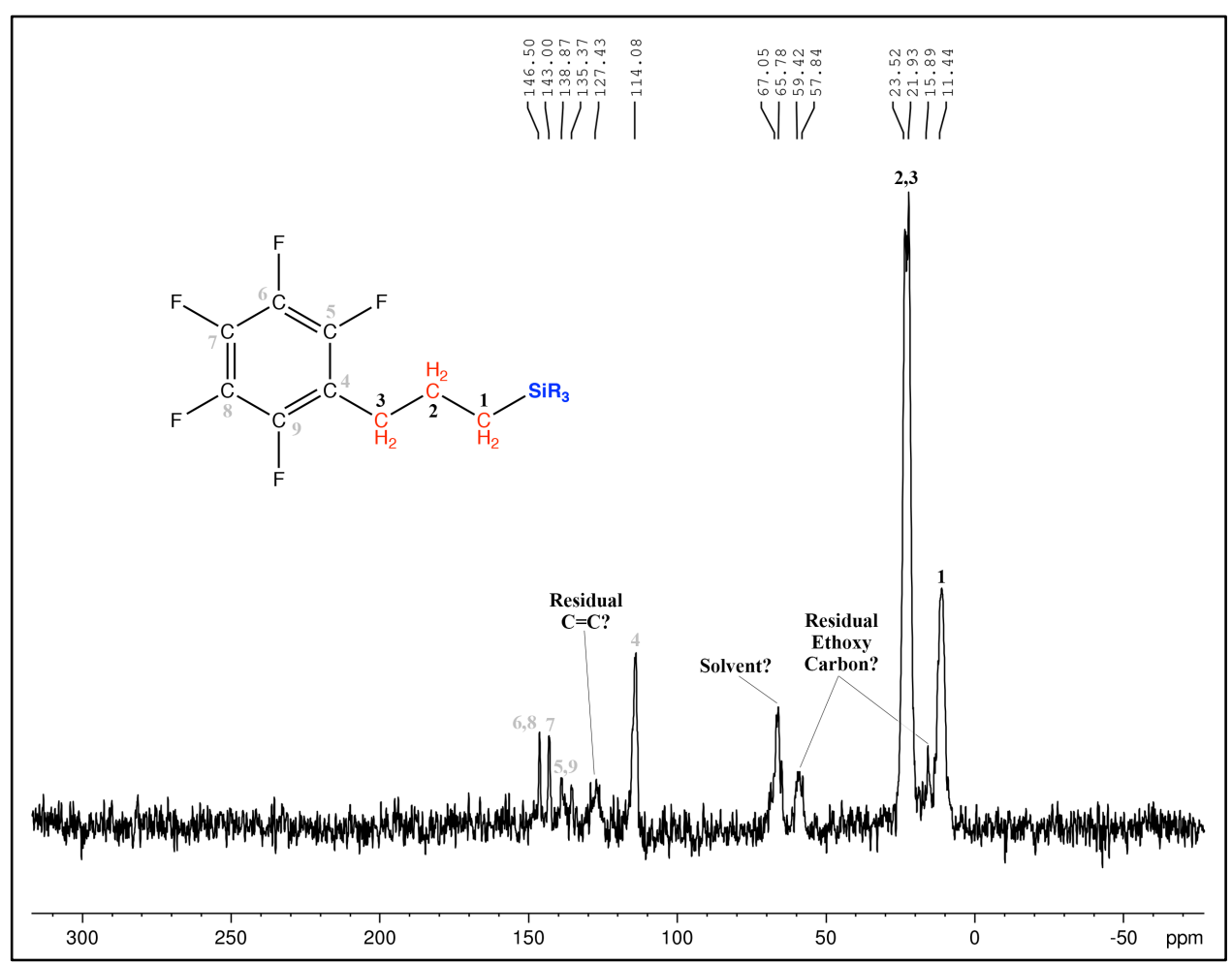

Figure 63. ${ }^{13} \mathrm{C}$ CP-MAS Solid-state NMR Spectra of PFP Hydride. 


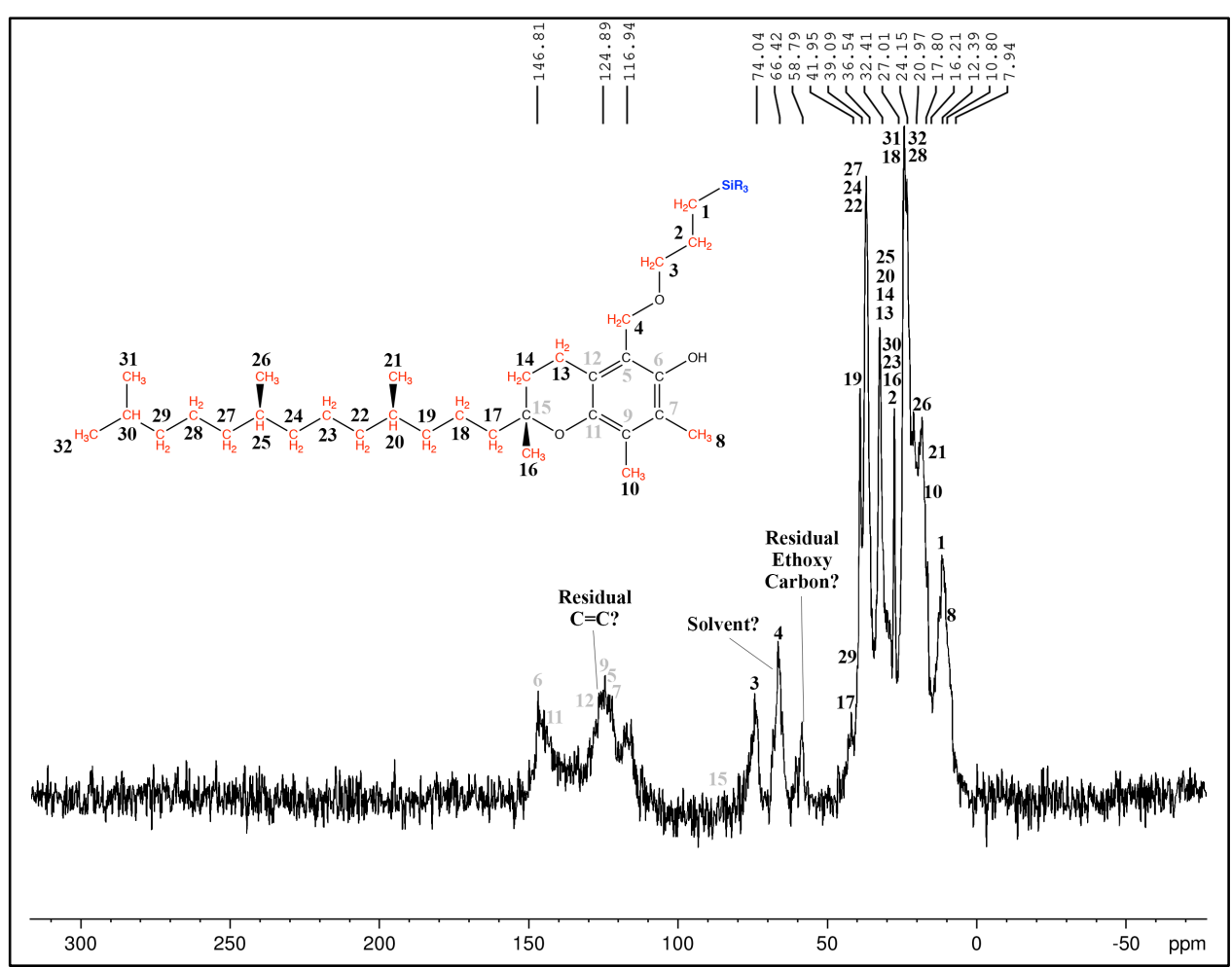

Figure 64. ${ }^{13} \mathrm{C}$ CP-MAS Solid-state NMR Spectra of TOCO Hydride.

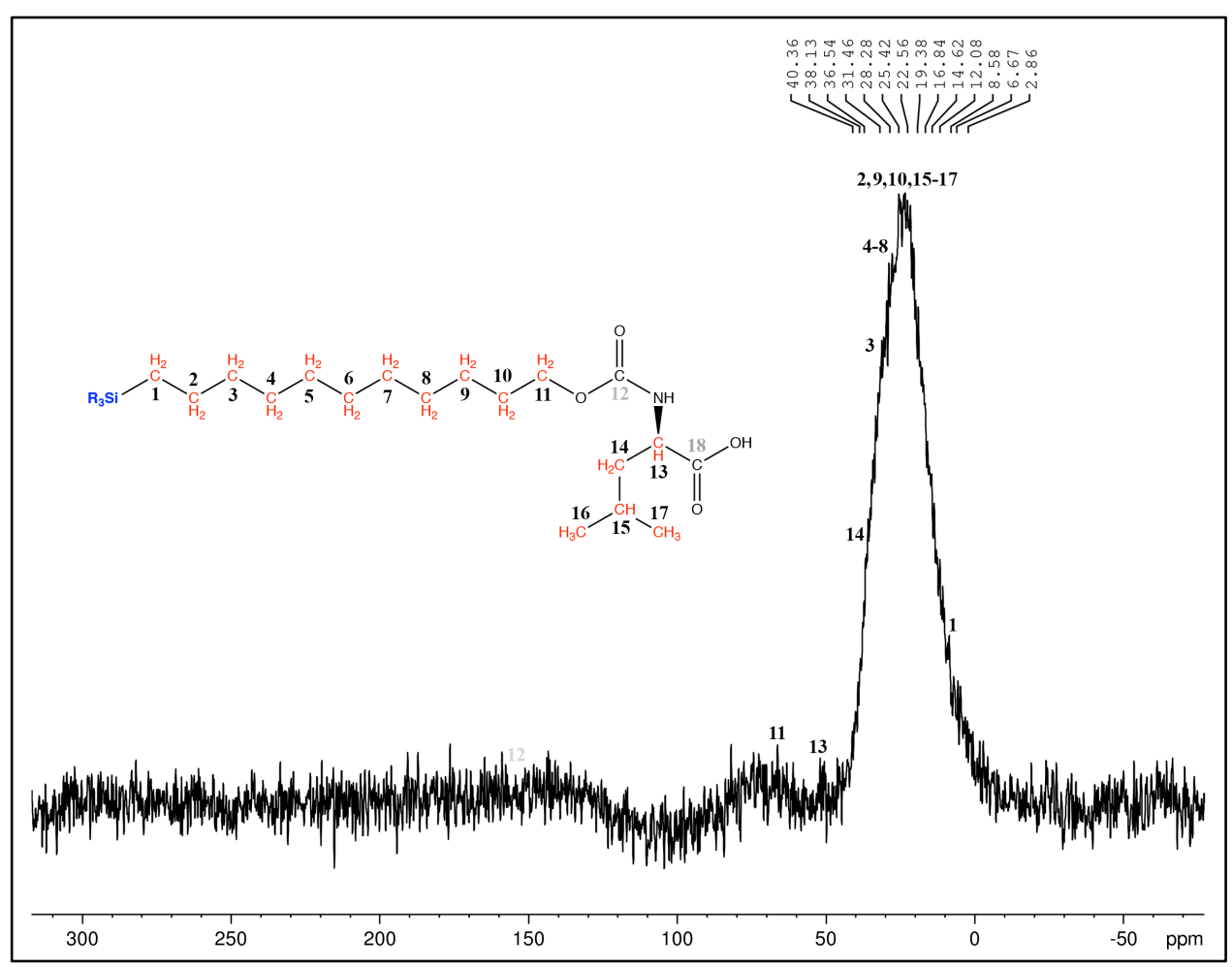

Figure 65. ${ }^{13} \mathrm{C}$ CP-MAS Solid-state NMR Spectra of LEU Hydride. 


\subsection{Column Performance Evaluation by HPLC-DAD}

The synthesized stationary phase materials were sent to MicroSolv Technologies, Inc. for packing into HPLC columns. Packing and quality control (QC) tests were conducted by Mr. Joshua Young for TOCO, PFP, and C20, and the most recently developed LEU column by Ms. Tanya Hiltz. The first three phases were packed individually in a $2.1 \mathrm{~mm}$ i.d. column, which is suitable for both HPLC-DAD and LC/MS experiments. The LEU column was packed in a $4.6 \mathrm{~mm}$ i.d. housing; therefore column characterization was only conducted in HPLC-DAD experiments (Figure 66, Table 16). Since the LEU column was developed nearly one year after the first three columns, only TOCO, PFP, and C20 were extensively characterized and studied for their applications.

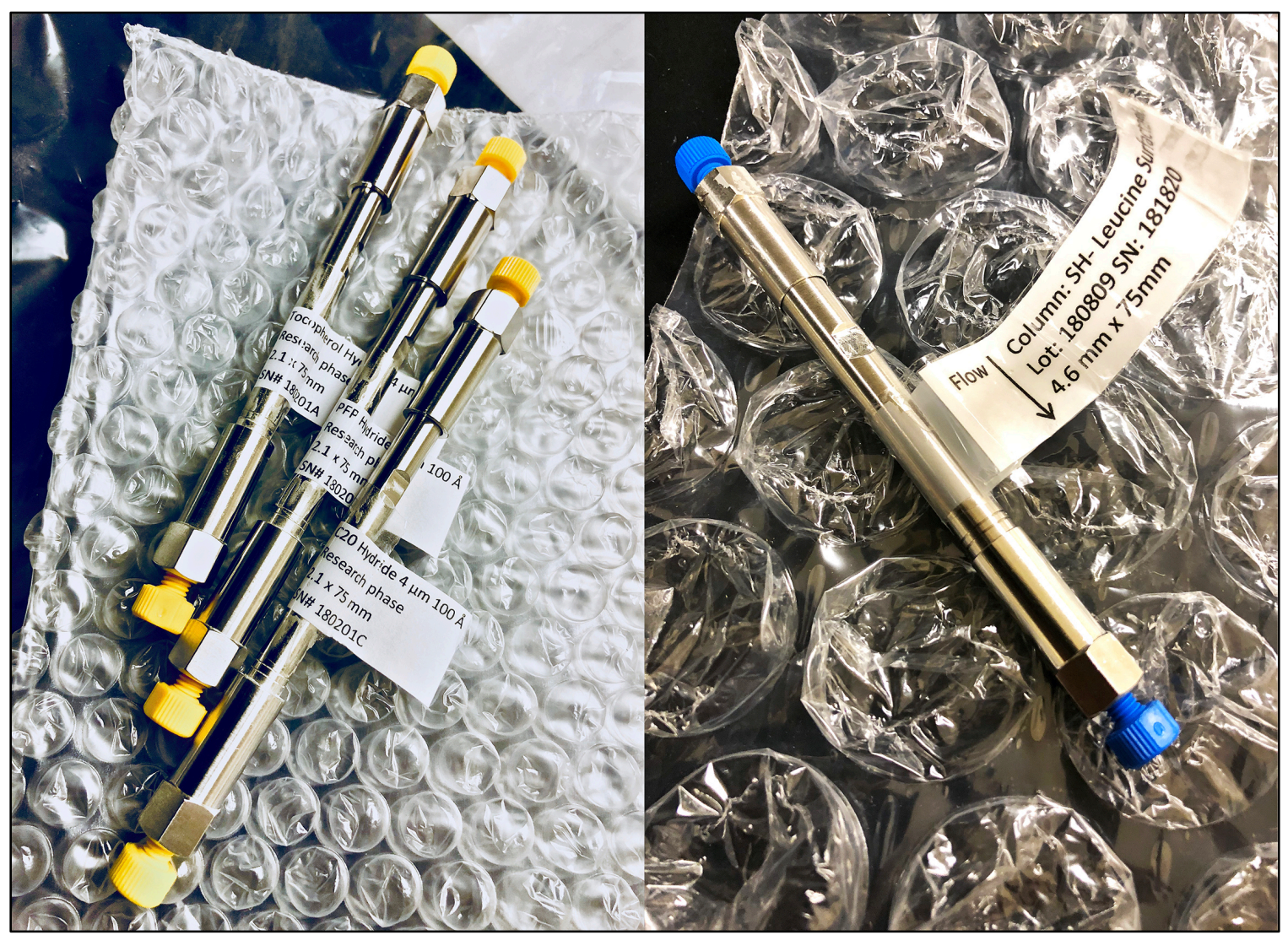

Figure 66. Newly Packed Columns (From Left: TOCO, PFP, C20, and LEU). 
Table 16. Specification of the Novel HPLC Columns.

\begin{tabular}{|c|c|c|c|c|c|}
\hline $\begin{array}{c}\text { Column } \\
\text { Name }\end{array}$ & Dimension & $\begin{array}{c}\text { Pore } \\
\text { Volume }\end{array}$ & $\begin{array}{c}\text { Particle } \\
\text { Diameter }\end{array}$ & Serial No. & Lot No. \\
\hline TOCO Hydride & $2.1 \times 75 \mathrm{~mm}$ & & & $180201 \mathrm{~A}$ & - \\
\hline PFP Hydride & $2.1 \times 75 \mathrm{~mm}$ & \multirow{2}{*}{$100 \AA$} & $4 \mu \mathrm{m}$ & $180201 \mathrm{~B}$ & - \\
\hline C20 Hydride & $2.1 \times 75 \mathrm{~mm}$ & & & $180201 \mathrm{C}$ & - \\
\hline LEU Hydride & $4.6 \times 75 \mathrm{~mm}$ & & & 181820 & 180809 \\
\hline
\end{tabular}

QC testing was conducted by injecting a mixture of uracil, pyridine, and phenol. This mixture can be used to assess void volume (uracil), silanol activity (pyridine), and hydrophobicity of the columns (phenol). The analytes were injected at $0.5 \mu \mathrm{L}$, eluted at $0.3 \mathrm{~mL} / \mathrm{min}$ with premixed (v/v) $90 \% \mathrm{DI} \mathrm{H}_{2} \mathrm{O} / 10 \%$ acetonitrile $/ 0.05 \% \mathrm{H}_{3} \mathrm{PO}_{4}$ and detected at $254 \mathrm{~nm}$. For the LEU column, the detection wavelength was $214 \mathrm{~nm}$ instead of $254 \mathrm{~nm}$. To account for increased internal diameter, the injection volume was raised to $1.0 \mu \mathrm{L}$ and the flow rate to $1.0 \mathrm{~mL} / \mathrm{min}$ (Figure 67 and 68 ).

As expected, all four columns show little to no retention for pyridine due to the lack of significant silanol activity towards basic molecules. The consistent retention of phenol indicates that all columns have the RP retention capability. It is also worth noting that the TOCO column showed slight retention for uracil $\left(t_{R}=1.014 \mathrm{~min}\right)$, and therefore pyridine is the least retained molecule $\left(t_{R}=0.795\right)$. This result indicates that TOCO likely has unique selectivity towards uracil and other analogous molecules. Furthermore, phenol retention by TOCO seems relatively weaker judging from its highest hydrophobicity value among all four phases $(\log \mathrm{P}=9.97)$, and the surface coverage value $\left(\alpha=1.269 \mu \mathrm{mol} / \mathrm{m}^{2}\right)$ similar to more polar LEU column $(\log \mathrm{P}=5.02, \alpha=1.286$ $\mu \mathrm{mol} / \mathrm{m}^{2}$ ) which has longer phenol retention. 


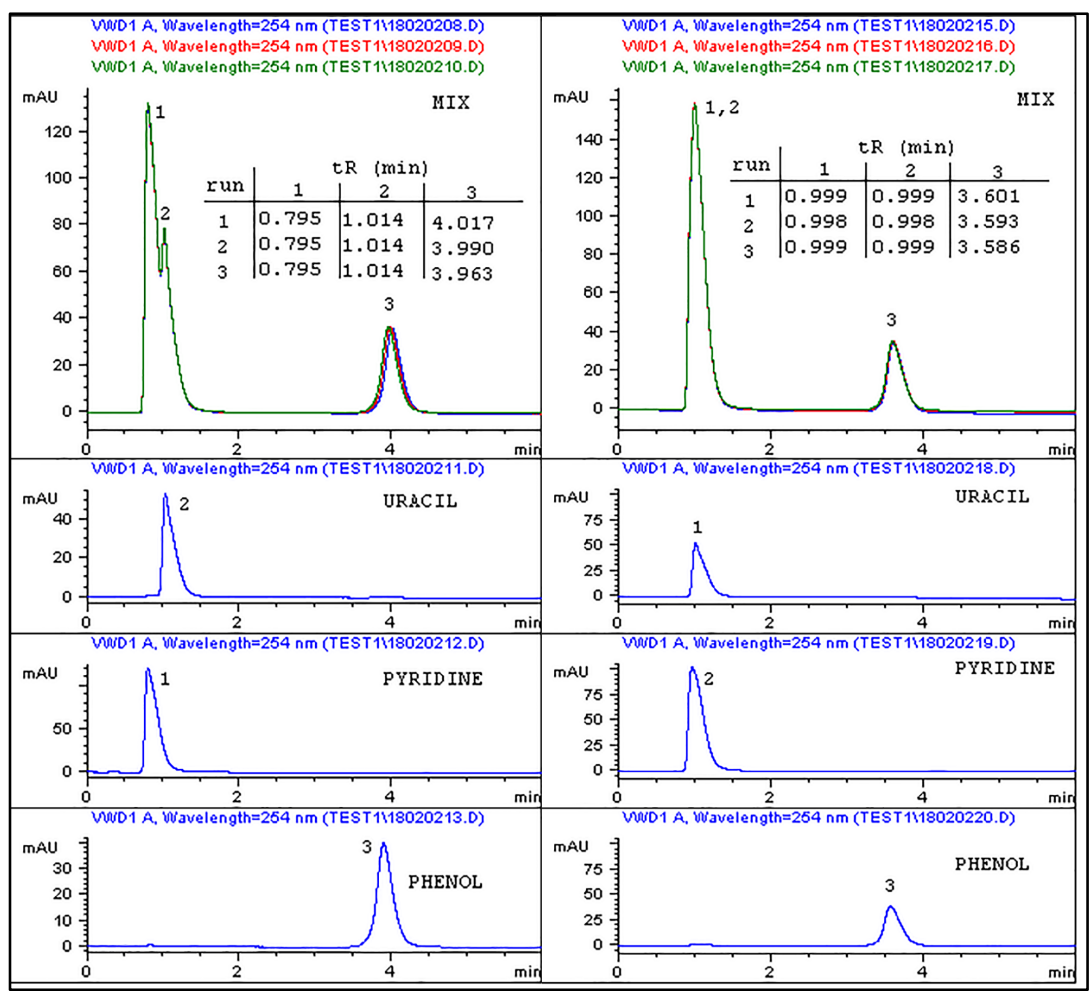

Figure 67. QC Test Results for TOCO (Left) and PFP (Right).

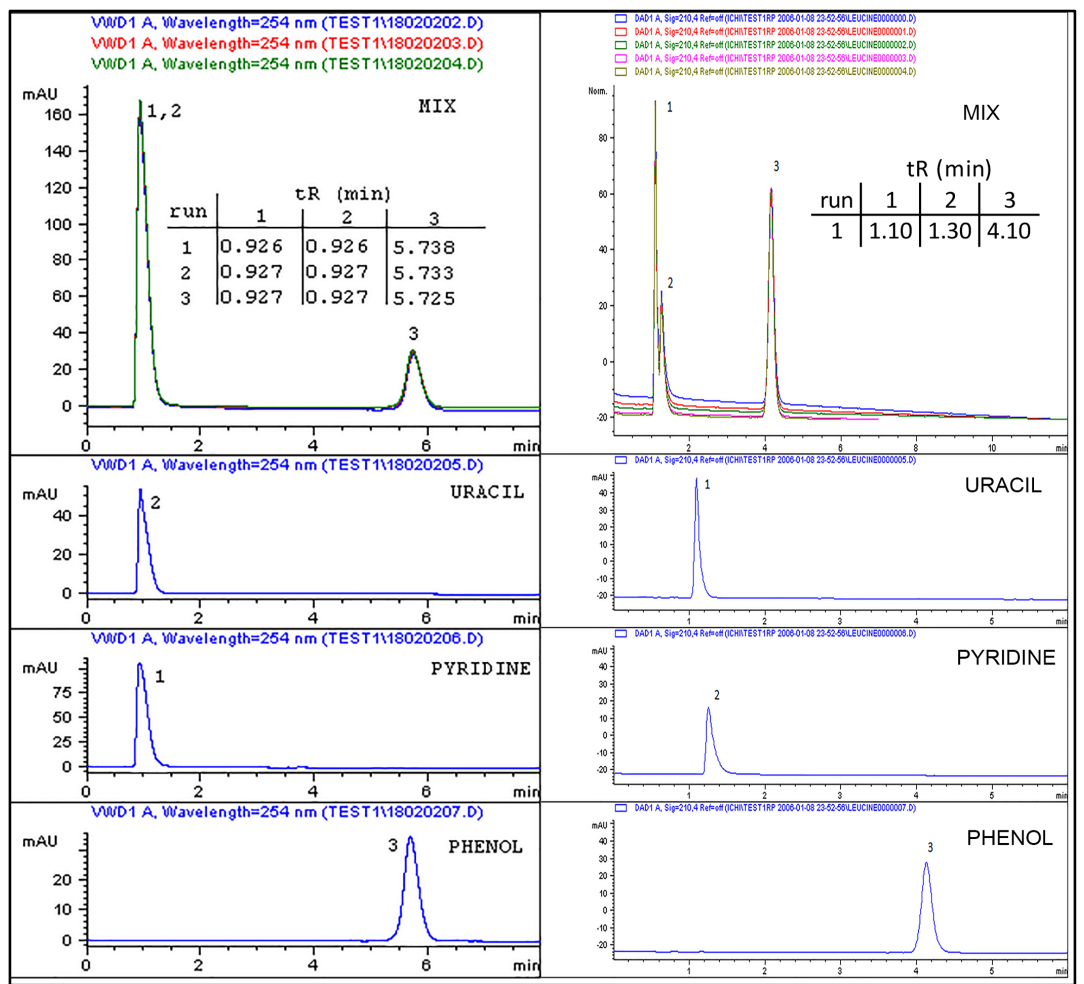

Figure 68. QC Test Results for C20 (Left) and LEU (Right). 


\subsubsection{SRM 870}

Analysis of Standard Reference Material ${ }^{\circledR} 870$ (SRM 870) has allowed better understanding of the general properties of four newly developed columns. Chromatographic conditions were adopted from the supplier's instruction manual. As a mobile phase, premixed $80 \%$ methanol/20\% $20 \mathrm{mM} \mathrm{pH} 7.0$ potassium phosphate buffer was used. For the $2.1 \mathrm{~mm}$ i.d. columns, the flow rate was $0.210 \mathrm{~mL} / \mathrm{min}$ and injection volumes were $1 \mu \mathrm{L}$. To maintain linear flow velocity, these parameters were increased for the $4.6 \mathrm{~mm}$ column to $1 \mathrm{~mL} / \mathrm{min}$ and $5 \mu \mathrm{L}$, respectively. Three detection wavelengths were used to determine the peak identity: $210 \mathrm{~nm}$ (for all five analytes), $254 \mathrm{~nm}$ (for toluene, ethyl benzene, and amitriptyline), and $480 \mathrm{~nm}$ (for quinizarin).

Chromatograms of the analysis results are shown in Figure 69. As previously mentioned, SRM 870 is primarily used for the evaluation of C18 RP columns. Thus, only the $\mathrm{C} 20$ column has exhibited the ability to resolve all five analyte compounds. Yet, this testing method should serve as a valid comparison and allows to elucidate the general properties of the columns as displayed in Table 17. The void volume is calculated by multiplying the flow rate by the retention time of the void marker - uracil. Although void volume measurement can be slightly controversial, the retention time of uracil still provides a good approximation of this parameter. Calculated values for the $2.1 \mathrm{~mm}$ i.d. columns range from $0.22-0.28 \mathrm{~mL}$, and roughly $1.0 \mathrm{~mL}$ for the $4.6 \mathrm{~mm}$ i.d. LEU column. Again, the TOCO phase showed slightly longer retention time for uracil at 1.35 mins. Methylene selectivity was only applicable to $\mathrm{C} 20$, which was the only column capable of resolving toluene and ethyl benzene at tested chromatographic conditions. 


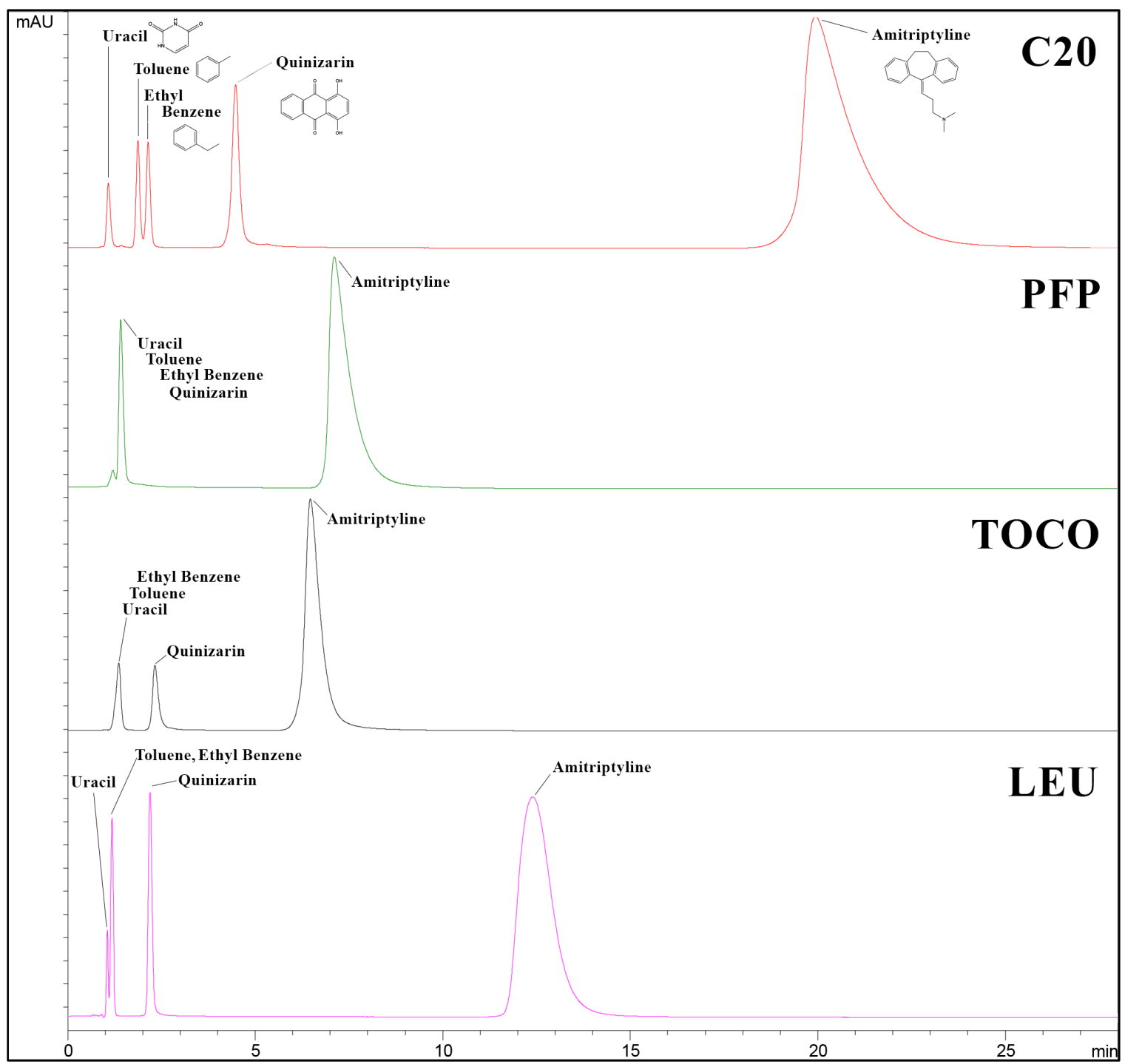

Figure 69. Chromatograms of SRM 870 Mixture Analysis.

Table 17. Evaluation Criteria in SRM 870 Test and Calculated Column Parameters.

\begin{tabular}{|c|c|c|c|c|}
\hline Column & $\begin{array}{c}\text { Void } \\
\text { Volume (mL) }\end{array}$ & $\begin{array}{c}\text { Methylene } \\
\text { Selectivity }\end{array}$ & $\begin{array}{c}\text { Metal Activity } \\
\text { (Quinizarin: N, TF) }\end{array}$ & $\begin{array}{c}\text { Silanol Activity } \\
\text { (Amitriptyline: } \text { N, As) }\end{array}$ \\
\hline C20 & 0.225 & 1.35 & $(7347,0.963)$ & $(3857,3.458)$ \\
\hline PFP & 0.252 & N/A & $(3080,0.689)$ & $(4262,3.961)$ \\
\hline TOCO & 0.284 & N/A & $(2898,0.671)$ & $(3525,1.865)$ \\
\hline LEU & 1.036 & N/A & $(7098,0.858)$ & $(1558,1.793)$ \\
\hline
\end{tabular}


The selectivity value here is the ratio of retention factor k' of two molecules. The metal and silanol activities of the columns seem to be moderate to low based on the observed peak shape and the three calculated parameters: theoretical plate $(\mathrm{N})$ and tailing factor $(\mathrm{TF})$ for quinizarin peak, and asymmetric factor $\left(\mathrm{A}_{\mathrm{S}}\right)$ for the amitriptyline peak. Procedures to calculate the two parameters are shown in Figure 70. A peak is considered symmetric when the TF or $A_{S}$ values are close to 1.

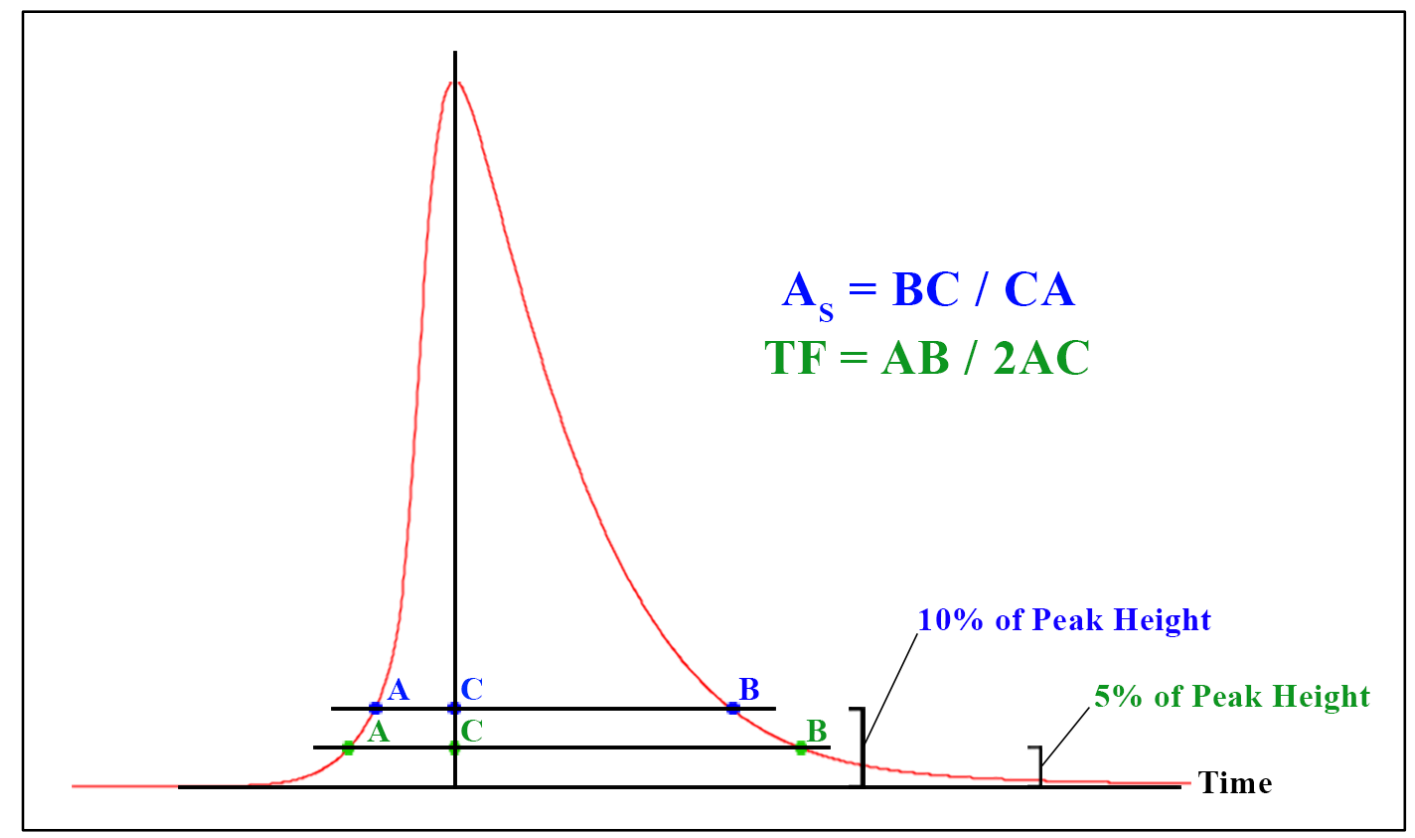

Figure 70. Calculation for Peak Asymmetric and Tailing Factor.

In summary, $\mathrm{C} 20$ exhibits the highest RP retentivity and methylene selectivity based on overall analyte retention and the separation of toluene/methylbenzene peaks. Although, further tests are necessary to elucidate the RP retention behaviors of the remaining three stationary phases. Since there are no significant tailing peaks from quinizarin and amitriptyline, all four columns have little to no metal and silanol activities. The slightly broad amitriptyline peak observed for C20 is likely due to the contribution from longitudinal diffusion effect caused by the long analyte retention. 


\subsubsection{Neue Test}

The Neue test was used to evaluate the hydrophobicity of columns by analyzing a mixture containing uracil $(16 \mu \mathrm{g} / \mathrm{mL})$ and two polycyclic aromatic hydrocarbons (PAHs; $60 \mu \mathrm{g} / \mathrm{mL}$ naphthalene and $200 \mu \mathrm{g} / \mathrm{mL}$ acenaphthene). The LEU column was not evaluated with this testing procedure. As a mobile phase, premixed 35\%-pH $720 \mathrm{mM}$ phosphate buffer $/ 65 \%$ methanol was used at a flow rate of $0.26 \mathrm{~mL} / \mathrm{min}$. A sample volume of $1 \mu \mathrm{L}$ was injected and detected at $214 \mathrm{~nm}$.

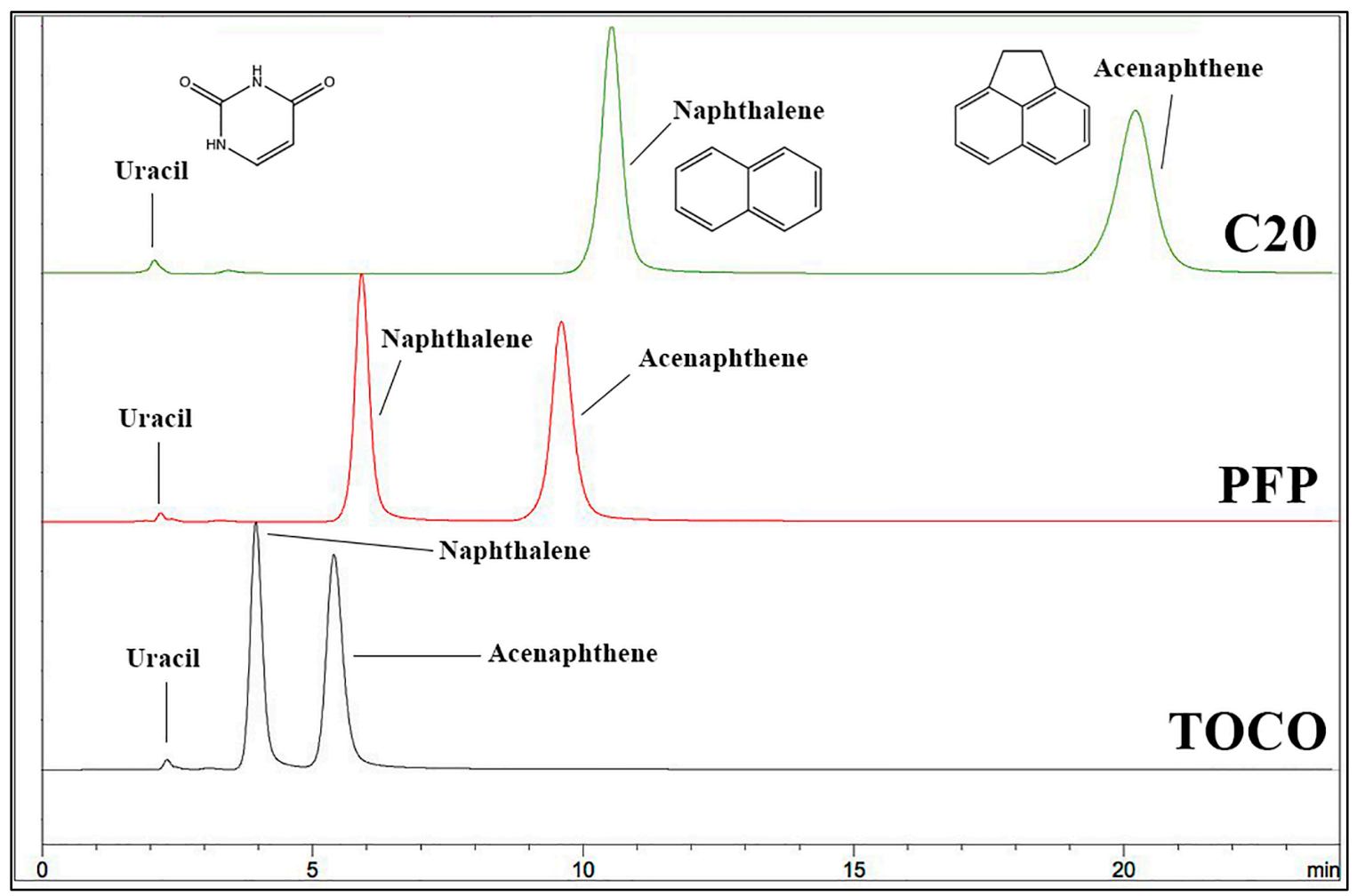

Figure 71. Neue Test for Column Hydrophobicity.

Neue test results are shown in Figure 71. The C20 column once again retains hydrophobic analytes the longest. While PFP and TOCO columns have weaker retentiveness compared to $\mathrm{C} 20$, both columns are capable of fully resolving the two molecules. Thus, all three columns can operate in the RP mode of separation. It is worth 
noting that the calculated hydrophobicity parameter $(v)$ for the three columns were considerably lower than the commercial C18 columns. While this is expected for the TOCO and the PFP columns, the initial plan for the $\mathrm{C} 20$ column $(v=0.0354)$ was to surpass the hydrophobicity parameter of a typical C18 columns (about $v=0.0400)($ Table 18). This is likely an outcome of C20's lower surface coverage values $\left(1.4 \mu \mathrm{mol} / \mathrm{m}^{2}\right)$ compared to typical commercial C18s (up to $3-3.5 \mu \mathrm{mol} / \mathrm{m}^{2}$ ).

Table 18. Neue Test - Hydrophobicity Parameter Comparison.

\begin{tabular}{|c|c|c|c|c|}
\hline Column & Hydrophobicity & $\mathbf{N}_{\text {Naphthalene }}$ & $\mathbf{N}_{\text {Acenaphthene }}$ & Selectivity \\
\hline C20 & 0.0354 & 9811 & 11538 & 2.15 \\
\hline PFP & 0.0290 & 5918 & 7559 & 1.99 \\
\hline TOCO & 0.0224 & 3897 & 3881 & 1.87 \\
\hline *Cogent BDC18 & 0.0397 & - & - & - \\
\hline *Cogent UDC Cholesterol & 0.0339 & - & - & - \\
\hline *Phenomenex Luna® C18 & 0.0400 & - & - & - \\
\hline \multicolumn{4}{|r|}{ *Values from MicroSolv Technologies, Inc. website } \\
\hline
\end{tabular}

\subsubsection{Dual Retention Properties}

The dual retention properties of the columns were investigated by measuring the capacity factors $(\mathrm{k})$ of polar/nonpolar/amphipathic molecules eluted at $10-100 \% \mathrm{~B}$ isocratic conditions. Mobile phases used were $\mathrm{DI} \mathrm{H}_{2} \mathrm{O}+0.1 \%$ formic acid (A), and acetonitrile $+0.1 \%$ formic acid (B). The sample volume of all analytes was $5 \mu \mathrm{L}$ volume and the concentration was $0.1 \mathrm{mg} / \mathrm{mL}$. Since the TOCO column shows slight retention towards the common void marker - uracil, potassium nitrate $\left(\mathrm{KNO}_{3}\right)$ was used instead to obtain the void time $-\mathrm{t}_{0}$ for $\mathrm{k}$ factor calculation. Elution was performed at 0.4 and 1.0 $\mathrm{mL} / \mathrm{min}$ for the $2.1 \mathrm{~mm}$ and $4.6 \mathrm{~mm}$ i.d. columns, respectively. Detection wavelength was set to $214 \mathrm{~nm}$ for $\mathrm{KNO}_{3}$ and $254 \mathrm{~nm}$ for caffeine, adenosine, adenine, and glyburide. 
Since the adenine retention profiles for TOCO/LEU were not interpretable due to poor peak shape, adenosine was used instead to test for polar analyte retention.

Primary RP retention is observed from glyburide $(\mathrm{CLogP}=3.53)$ and ANP retention from adenosine or adenine $(\mathrm{C} \log \mathrm{P}=-4.77$ and -3.38 , respectively). Weak retention for both modes were observed for caffeine $(\mathrm{C} \log \mathrm{P}=-1.375)$.

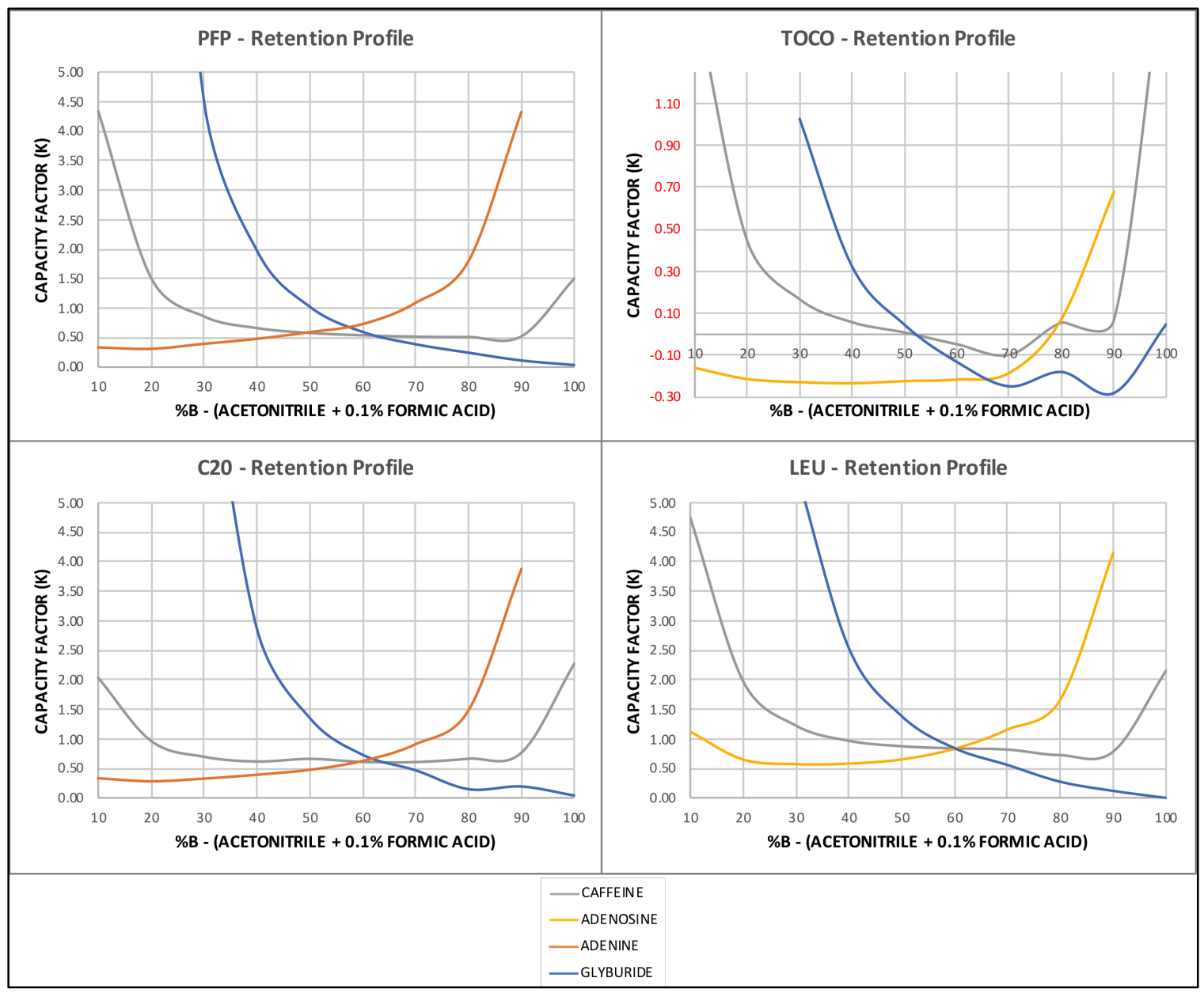

Figure 72. Retention Profiles of the Novel Hydride-based Stationary Phases.

As shown in Figure 72, all four columns exhibit "U-shaped" retention profiles which verifies the dual retention capabilities of the columns. The lowest average retention time of the three analytes are observed between 55 to $65 \%$ B. Although, the point of 
convergence seems to shift towards the lower $\% \mathrm{~B}$ direction as the molar volume of the bonded ligand decreases: TOCO $(65 \% \mathrm{~B}, 154.78 \mathrm{~cm} 3 / \mathrm{mol})>\mathrm{C} 20(62.5 \% \mathrm{~B}, 94.31$ $\left.\mathrm{cm}^{3} / \mathrm{mol}\right)>\operatorname{LEU}\left(60 \% \mathrm{~B}, 92.1 \mathrm{~cm}^{3} / \mathrm{mol}\right)>\operatorname{PFP}\left(57.5 \% \mathrm{~B}, 42.45 \mathrm{~cm}^{3} / \mathrm{mol}\right)$. The TOCO phase once again has the lowest overall retentivity as observed in the previous test results.

\subsubsection{Dimethoxybenzene Selectivity}

Selectivity of methoxybenzene regio-isomers was evaluated by a mixture of anisole and dimethoxybenzenes (DMB). The ability of columns to chromatographically distinguish these molecules can be potentially beneficial for chemical analysis in food science and pharmaceuticals. This is because many natural or artificially made biologically active molecules consist of lipophilic benzene ring(s) regio-selectively bonded to various functional groups.

The DMB analysis was conducted using DI water $+0.1 \%$ formic acid (A) and acetonitrile $+0.1 \%$ formic acid as mobile phases. The column oven temperature was set to $40^{\circ} \mathrm{C}$, the flow rate to $0.3 \mathrm{~mL} / \mathrm{min}$, and the detection wavelength at $280 \mathrm{~nm}$. The gradient condition was: $0.0-1.0 \mathrm{~min} 5 \% \mathrm{~B} ; 1.0-12.0 \mathrm{~min}$ to $95 \% \mathrm{~B} ; 12.0-14.0 \mathrm{~min} 95 \%$ $\mathrm{B} ; 14.1-14.1 \mathrm{~min}$ to $5 \% \mathrm{~B} ; 14.0-15.0 \mathrm{~min}$ to $5 \% \mathrm{~B}$, with 7 minutes of post time after each run. The phenyl hydride column was used for comparison with the PFP column.

Using these chromatographic conditions, the DMB selectivity and retention are considerably better for PFP than a non-fluorinated phenyl column (Figure 73). This is likely due to the unique selectivity given by highly electronegative Fluorine atoms. The 
PFP ligand is known to provide a stationary phase with an ability to participate in dipoledipole and induced dipole-dipole interactions with neutral analytes such as DMBs.

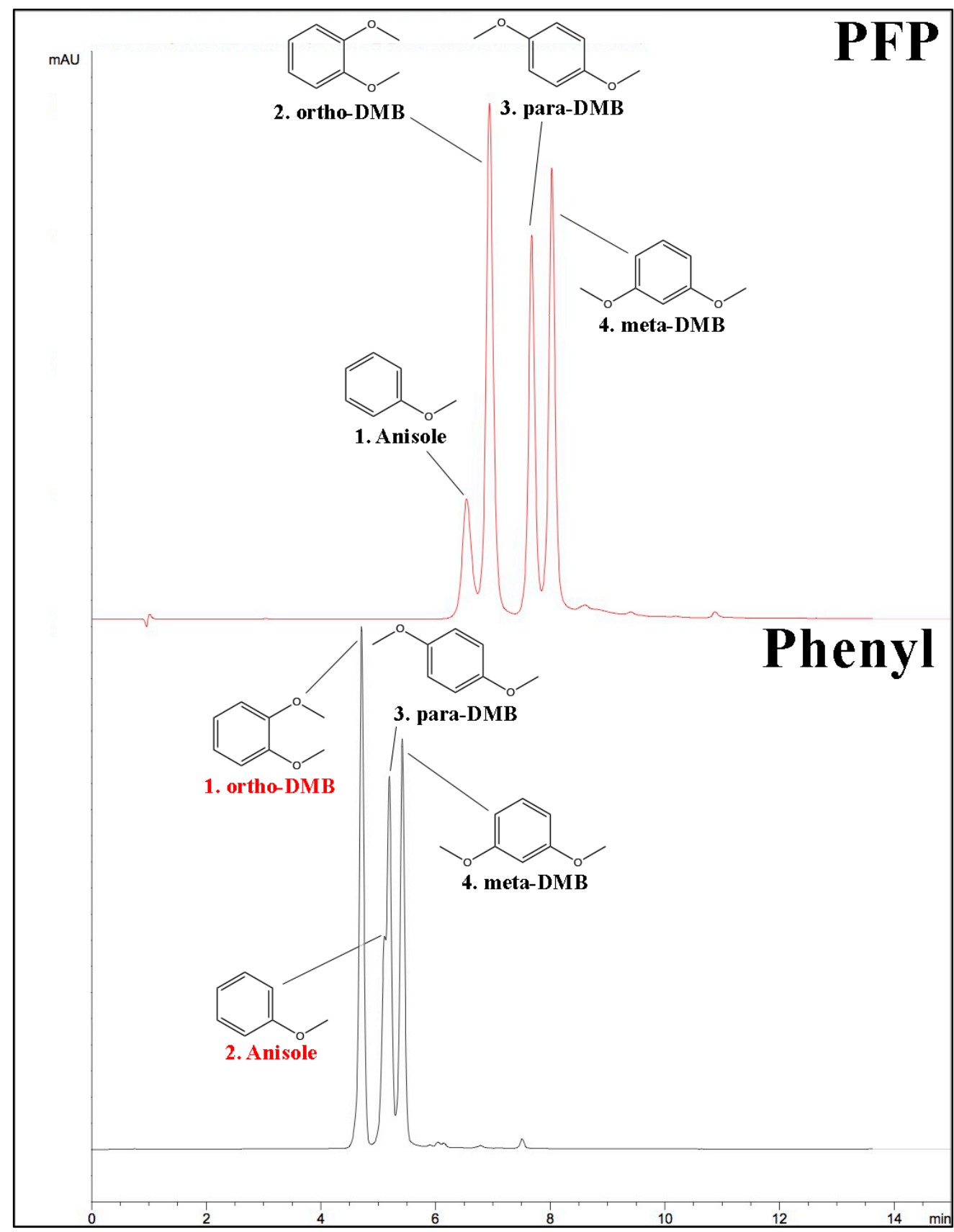

Figure 73. DMB Selectivity Evaluation for the Aromatic Columns.

Furthermore, the PFP column has different retention order from the phenyl column where anisole and o-DMB were switched. 
Separation of the methoxybenzenes by other phases were not as efficient as the PFP column (Figure 74). TOCO and C20 columns were unable to resolve all four analytes; however, the LEU column was able to achieve partial separation on most of the methoxybenzene analogs. In fact, the peak separation for $\mathrm{m}-\mathrm{DMB}$ and $\mathrm{p}-\mathrm{DMB}$ was comparable to the PFP column.

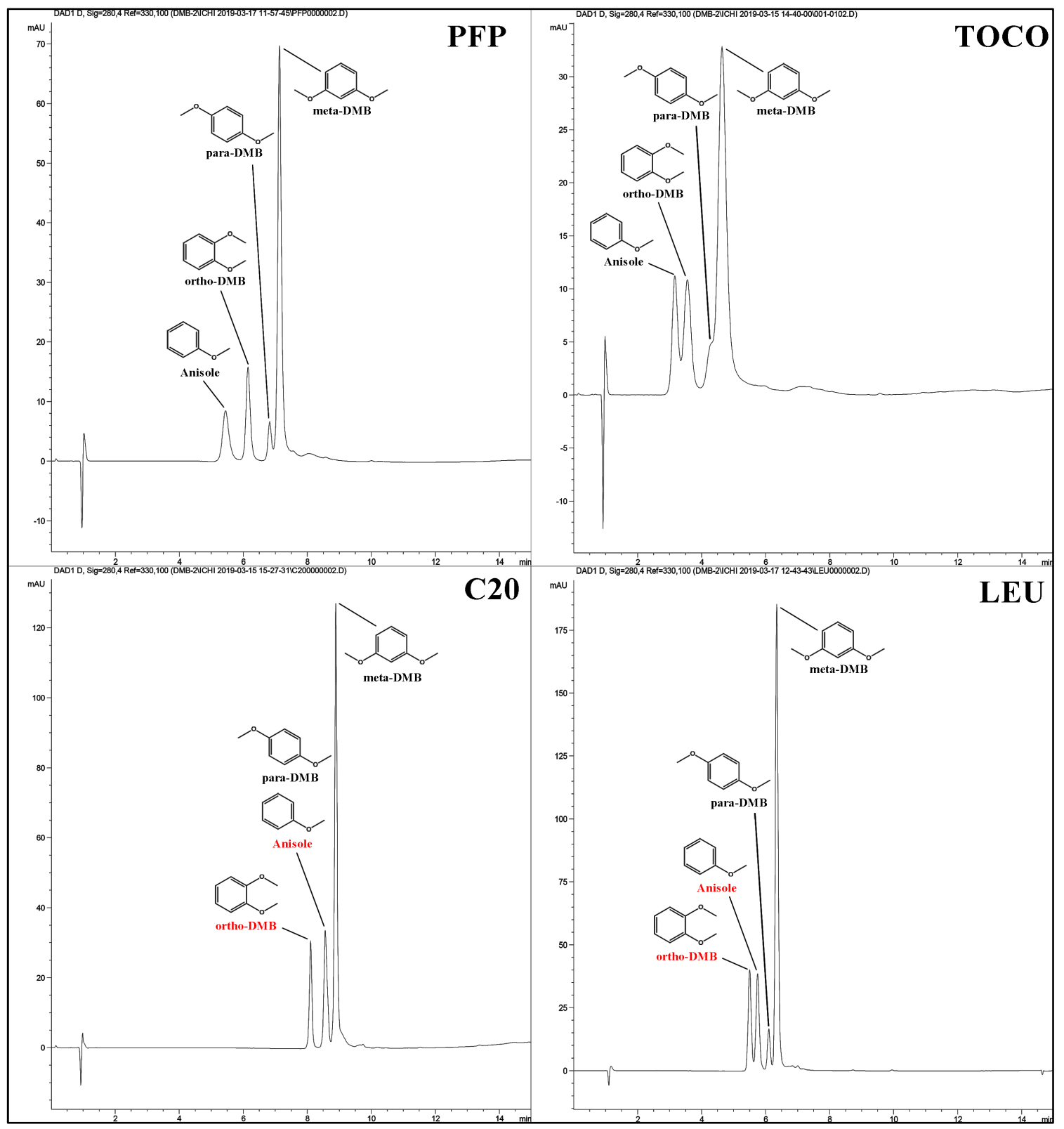

Figure 74. DMB Selectivity Evaluation for All Four Stationary Phases. 


\subsection{Detection of PCBNs in Formulation and Cannabis Plant Extracts}

There are hundreds of phytocannabinoids (PCBNs) present in cannabis plants but only a few are considered and used as active pharmaceutical ingredients (API). Cannabidiol (CBD) and tetrahydrocannabinol (THC) are two isobaric $(315 \mathrm{~m} / \mathrm{z}-$ $[\mathrm{M}+\mathrm{H}]^{+}$) and most highly abundant APIs in cannabis plants [83]. In fresh plants, CBD and THC are present as carboxylic acid derivatives (CBDA and THCA, $359 \mathrm{~m} / \mathrm{z}-$ $[\mathrm{M}+\mathrm{H}]^{+}$) which gradually undergo decarboxylative conversion to target API molecules (e.g., over time, or by heating) $[84,85]$. The presence of CBD was confirmed by reference standards, and other molecules of interest were only tentatively identified due to the lack of reference materials.

The gradient method used in this project was: $0.0-0.5 \mathrm{~min}$ at $10 \% \mathrm{~B} ; 0.5-1.5 \mathrm{~min}$ to $50 \% \mathrm{~B} ; 1.5-2.5 \mathrm{~min}$ at $50 \% \mathrm{~B} ; 2.5-3.5 \mathrm{~min}$ to $90 \% \mathrm{~B} ; 3.5-4.5 \mathrm{~min}$ at $90 \% \mathrm{~B} ; 4.5-5.0$ min to $10 \% \mathrm{~B} ; 5.0-7.0 \mathrm{~min}$ at $10 \% \mathrm{~B}$, with 4 minutes of post time after each run. Mobile phases used were DI water $+0.1 \%$ formic acid (A) and acetonitrile $+0.1 \%$ formic acid (B). For all sample analyses $0.4 \mathrm{~mL} / \mathrm{min}$ flow rate and $4 \mu \mathrm{L}$ injection volume were used. Sample detection was performed on an Agilent 6200 Series dual-ESI-TOF MS analyzer in the positive ionization mode from $100-3000 \mathrm{~m} / \mathrm{z}$ range.

Three columns (TOCO, PFP, and C20) were first evaluated for the retentivity of CBD in the gummy-edible sample prepared by solid-phase extraction (SPE) (Figure 75). All three columns were able to produce reasonable retention time and peak shape. Further analyses were conducted using the $\mathrm{C} 20$ column which achieved the longest $\mathrm{CBD}$ retention among the three columns using the above gradient condition. 


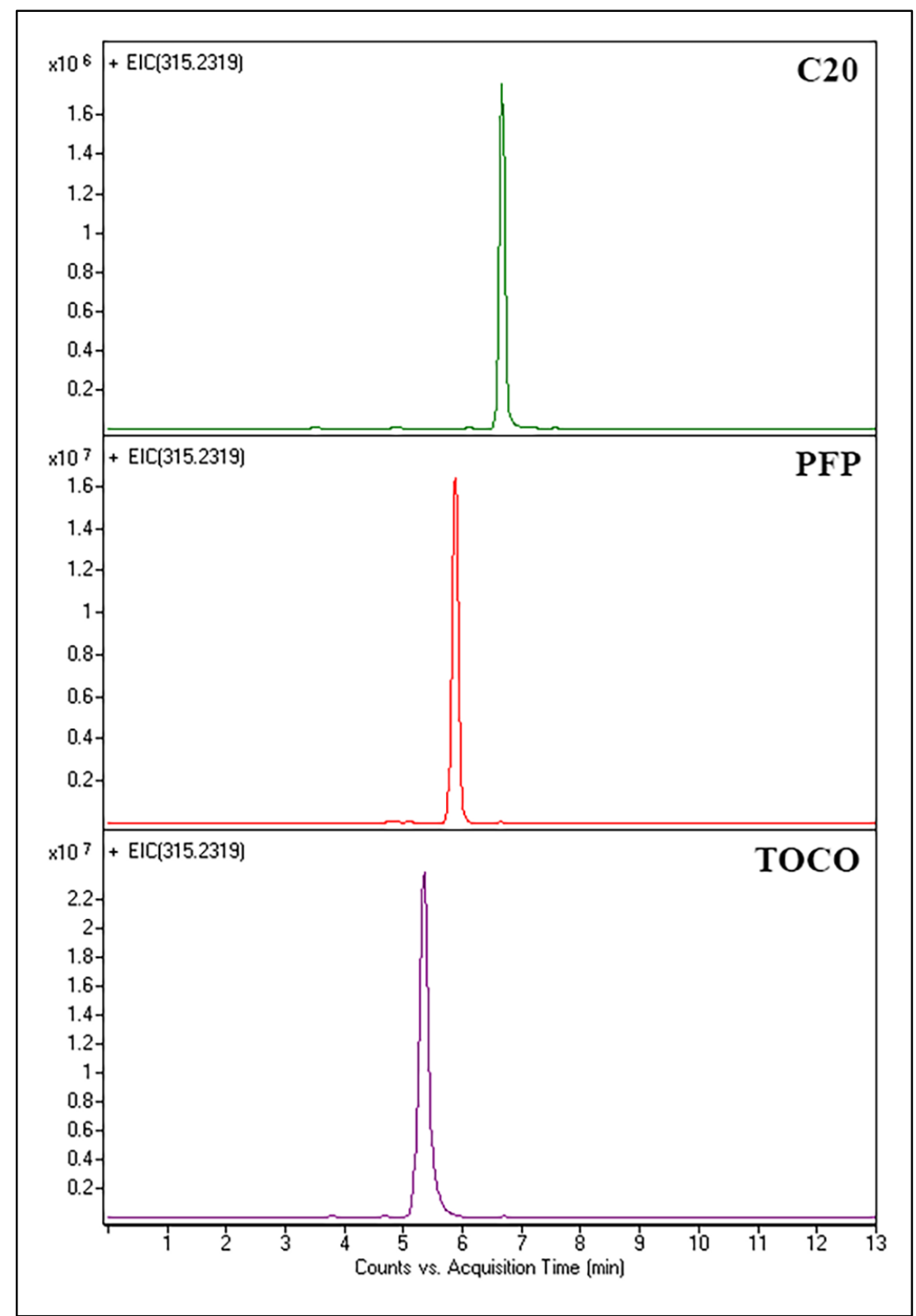

Figure 75. CBD Detected from the SPE-processed Gummy Edible.

The extracts of four cannabis plants were qualitatively analyzed for the presence of CBD. However, no peak was observed at the expected retention time window for the extracted ion chromatogram (EIC) at $315 \mathrm{~m} / \mathrm{z}$. Lack of CBD was further confirmed by the isotope-labelled d3-CBD internal standard (IS; at $318 \mathrm{~m} / \mathrm{z}$ ) spiked into every cannabis 
samples (Figure 76). The isobaric analyte peak and the carboxylate derivative peak (359 $\mathrm{m} / \mathrm{z}$ ) observed at later times in the chromatograms likely correspond to other major PCBNs component such as THC and $\mathrm{CBC}$ and acid derivatives of PCBNs, respectively. However, the identity of these peaks cannot be determined without the reference standards or MS/MS fragmentation study.

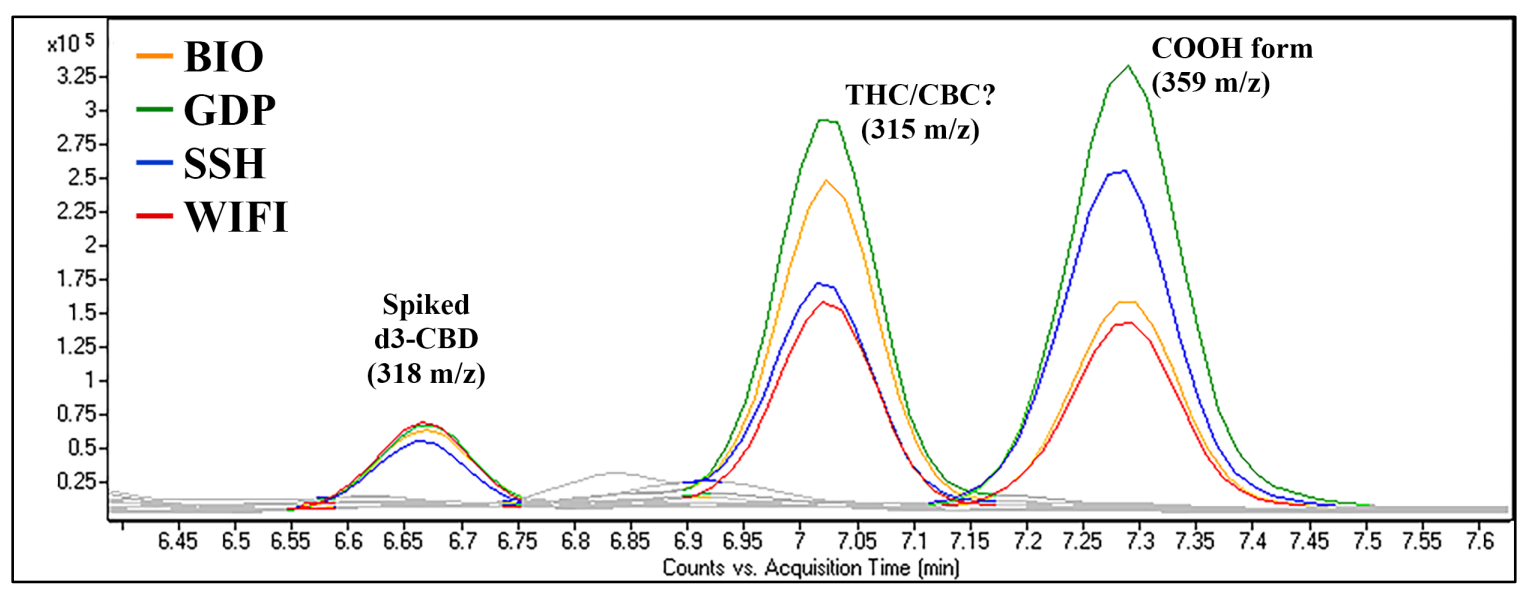

Figure 76. Overlaid Chromatograms of Four Cannabis Plant Extracts.

Therefore, quantification was performed only for CBD in the gummy-edible sample.

Figure 77 shows the CBD calibration curve prepared from 0.1 to $100 \mu \mathrm{g} / \mathrm{mL}$ concentration $\left(\mathrm{R}^{2}=0.9958\right)$. The green peak represents the 10 -fold diluted edible sample, the red peak is the $\mathrm{d} 3-\mathrm{CBD}$ spiked into all samples analyzed in the study, and the gray peak shows the signal intensity of calibration points. The standard curve was generated in GraphPad PRISM by using the analyte-to-IS peak area ratio and fitting with quadratic regression and 1/y weighting. The concentration of gummy-edible was calculated to be $60.0 \mu \mathrm{g} / \mathrm{mL}$, which was about $20 \%$ recovery from the actual value. The majority of CBD was lost most likely during the SPE process. The limit of quantitation (LOQ; signal-to-noise ratio $(\mathrm{S} / \mathrm{N})=10)$ and detection $(\mathrm{LOD} ; \mathrm{S} / \mathrm{N}=3)$ were calculated to 
be 30.3 and $9.1 \mathrm{ng} / \mathrm{mL}$, respectively. There are two possible explanations to the absence of CBD in cannabis extracts: (1) because the concentration was below the LOD, and/or (2) because CBD is present as its acidic precursor CBDA, as mentioned above.

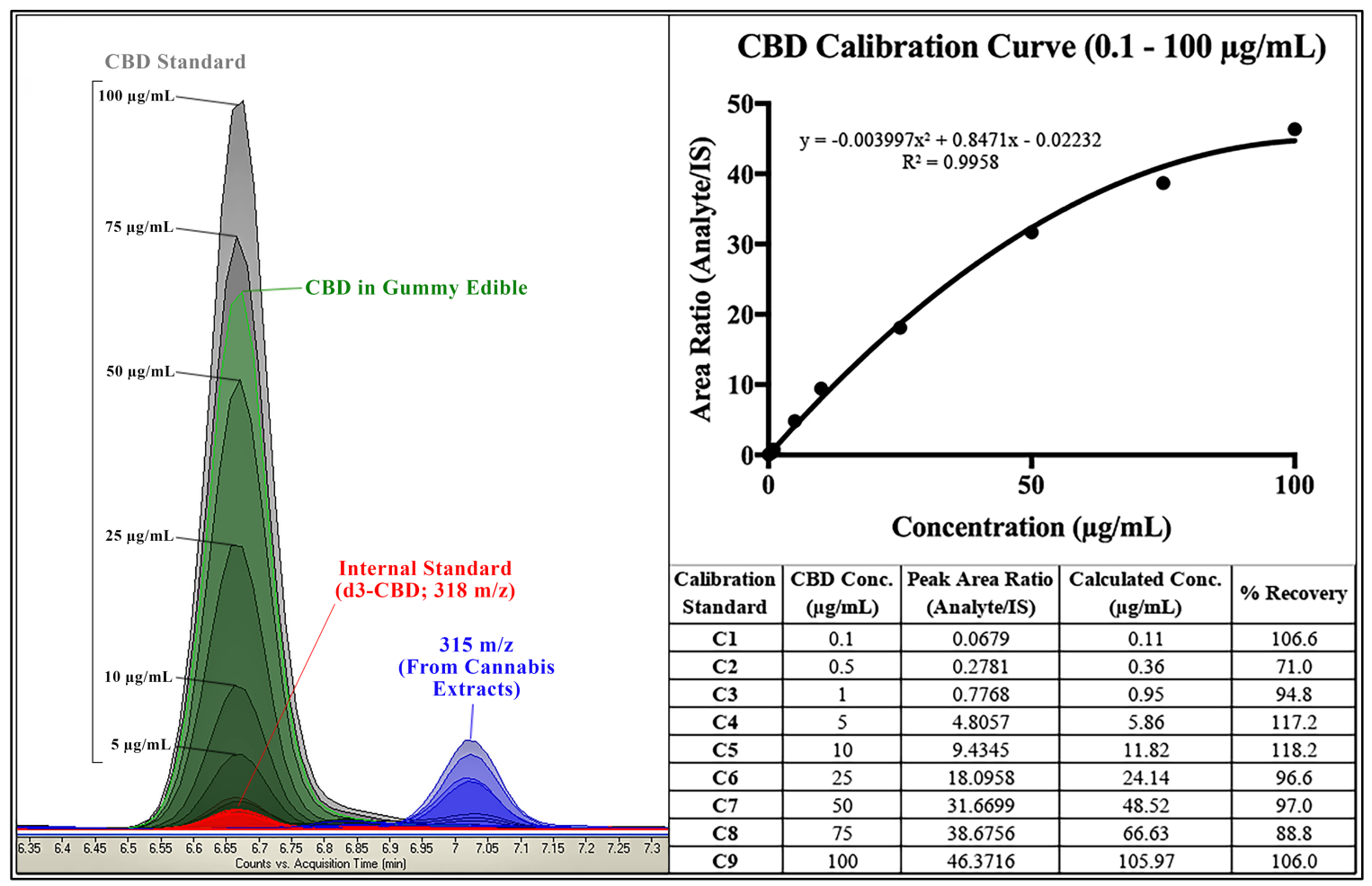

Figure 77. Calibration Curve for CBD Quantification.

\subsection{Retention Profile of Controlled substances}

The chromatographic performance of three experimental columns (TOCO, PFP, C20) were further assessed by investigating the retention behavior of commonly abused drugs in human urine samples and neat solutions. Biological samples such as urine and blood plasma contain matrix components (e.g., salts, phospholipids) that tend to interfere with the ionization process of analyte molecules in an ESI source. The SPE process used for the urine and CBD edible samples essentially removes the matrix in the sample while concentrating the analyte compounds of interest in eluate. 


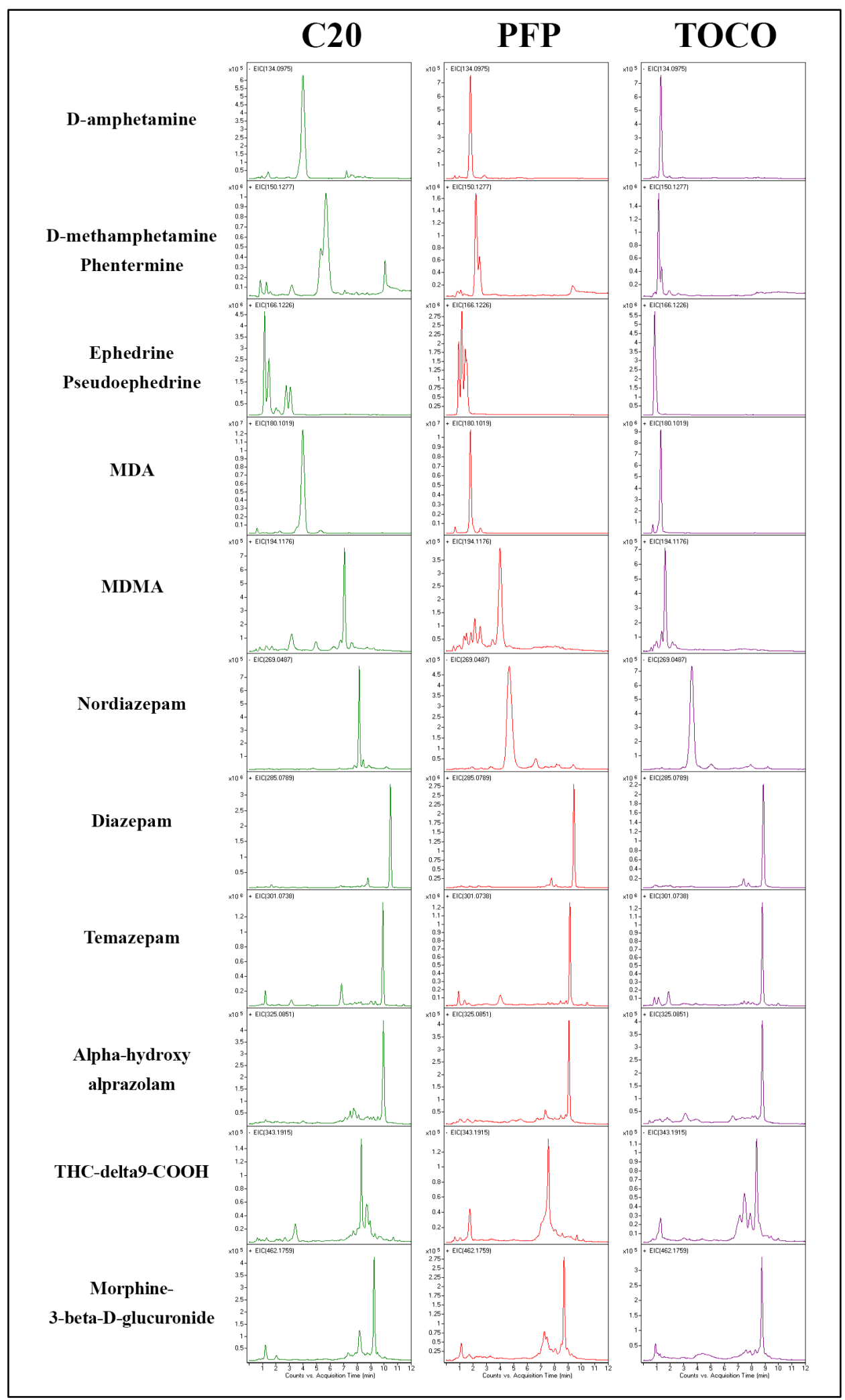

Figure 78. Retention Profiles of Eleven Illicit Substances Detected in Human Urine. 
Figure 78 shows the chromatograms of thirteen out of nineteen substances that were SPE-extracted at detectable levels. The other six substances (benzylpiperazine, benzoylecgonine, oxazepam, 6-monoacetylmorphine, and codeine) were undetected due to low ionization efficiency and/or insufficient recovery from the SPE procedures. Most analytes had reasonable retention and symmetric peaks. However, two isobaric analyte pairs (D-methamphetamine/phentermine and ephedrine/pseudoephedrine) remained unresolved by all three columns. THC-delta9-COOH (or THCA) was also eluted as a poorly shaped peak. Surprisingly, morphine-3-beta-D-glucuronide (M3BG) was one of the few compounds well-retained by TOCO when compared to C20. For other analytes, C20 had the longest retention followed by the PFP column except for M3BG.

Eight additional drugs of abuse in solution were further analyzed to evaluate the analyte selectivity by the three columns. Five of these were cathinone-class drugs (cathinone, 4-fluoromethcathinone, N-ethylcathinone, mephedrone, and pentedrone), a group of psychoactive designer drugs commonly known as "bath salts". All five cathinones are classified as DEA schedule I along with three other substances: LSD, heroin, and MDMA. Chromatograms in Figure 79 show that C20 and PFP can easily separate the cathinone drugs. However, MDMA $(\mathrm{CLogP}=1.685)$ and mephedrone $(\mathrm{CLogP}=1.683)$ were only partially separated by the two columns. Since the heroin signal was considerably lower than other analytes, the EIC of heroin is displayed separately in a magnified scale in Figure 80. 


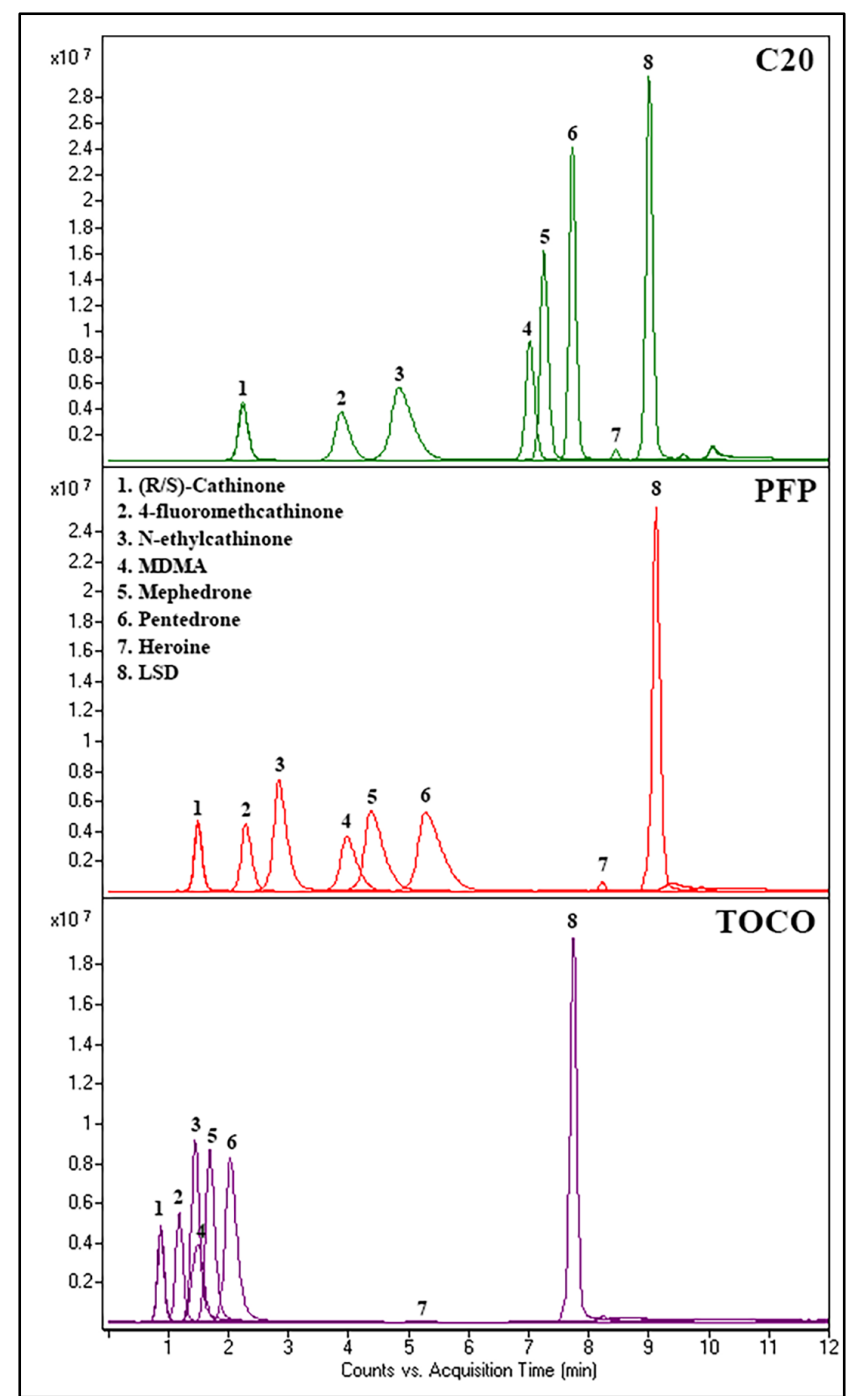

Figure 79. Retention Profile of Eight Commonly Abused Drugs in Neat Solution.

The TOCO column had an interesting behavior where relatively small molecules (e.g., cathinones and MDMA) were weakly retained but heteroaromatic/polycyclic molecules (e.g., LSD and heroin) were well-retained and often more efficiently separated than C20/PFP columns [86]. A similar trend has been observed in human urine analysis as well, where multi-ring compounds such as THCA, morphine glucuronide, and heterocyclic benzodiazepine class drugs were very well-retained. 


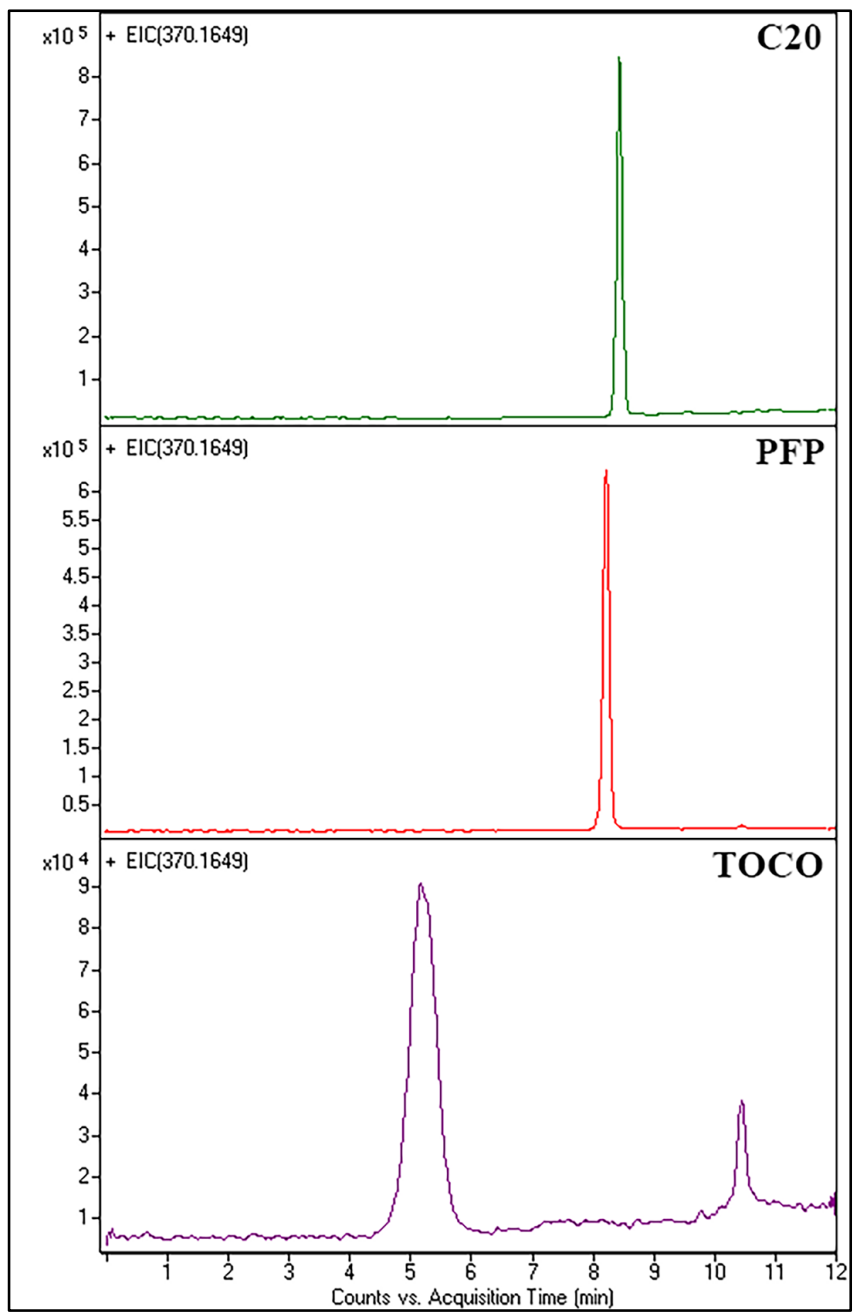

Figure 80. EIC of Heroin in Neat Solution at Magnified Scale.

\subsection{Metabolomics Characterization of USDA Grape Cultivars}

In metabolomics studies, metabolite abundance within biological systems are profiled in targeted and/or non-targeted manner. Thus, silica hydride's ability to achieve retention and separation in a broad range of analyte polarity is highly advantageous. In this project, wine grape (Vitis vinifera) is used as a model organism and metabolite components in five grape skin extracts obtained from the USDA grape cultivars are analyzed and compared. Subjects are: Scarlet Royal (SR), C33-30 (C33), Y144-162-12 (\#162), Y144-169-12 (\#169), and Y539-194-06 (Y539). SR and C33 are two red 
seedless table grapes (RSTGs) where C33 is the mother of USDA-patented cultivar SR, which was produced by crossbreeding with other RSTGs. The other three selections are also in a parent-daughter relationship, where Y539 (male) was crossed with a red-fleshed 06-7502-4 (female; not studied) to produce the sibling selections named \#162 and \#169. The \#162/\#169 grapes are both red-fleshed breeds like their mother, while Y539 is not.

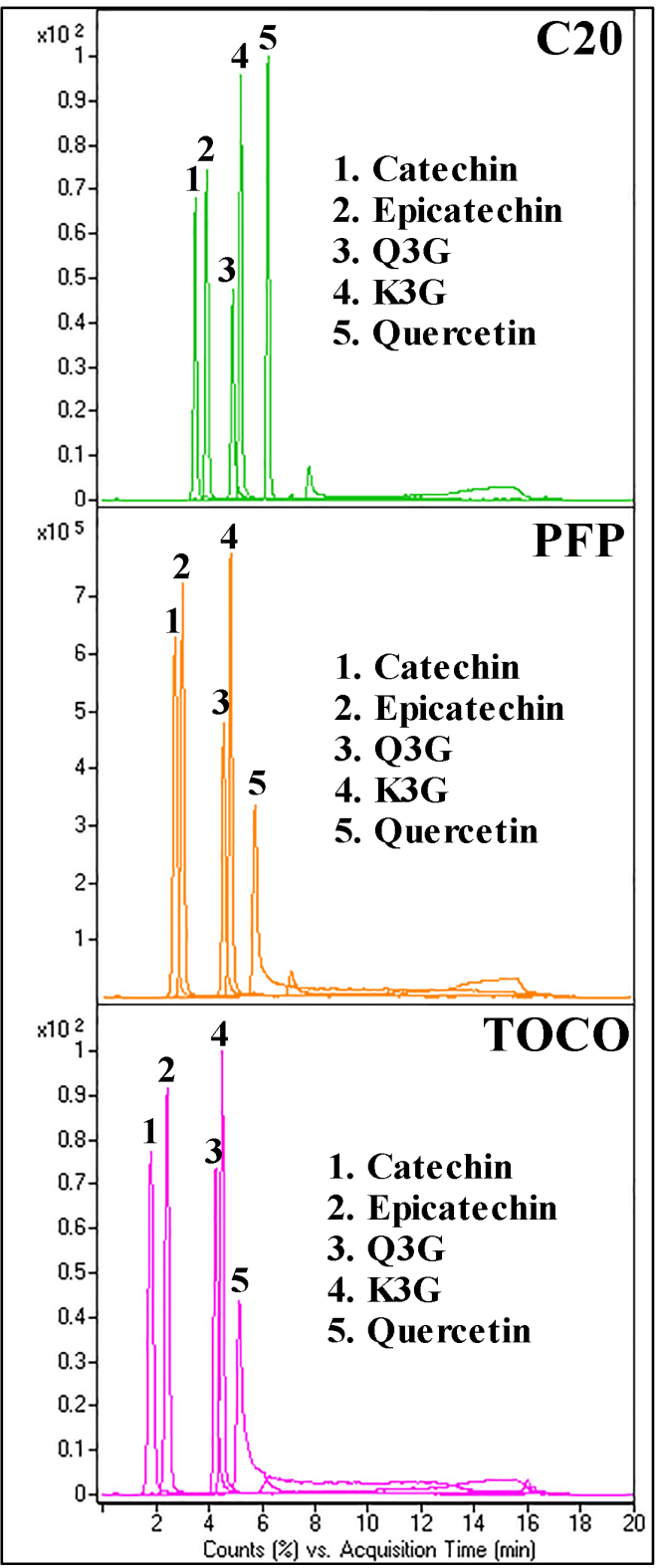

Figure 81. Retention Profile of Five Flavonoid Reference Standards. 
Before the grape skin extracts were analyzed, five flavonoid molecules commonly found in wine were assessed on the three columns (TOCO, PFP, C20). Mobile phases used were: $\mathrm{DI} \mathrm{H}_{2} \mathrm{O}+0.1 \%$ formic acid (A) and acetonitrile $+0.1 \%$ formic acid (B). Skin extracts were injected at a $5 \mu \mathrm{L}$ volume and eluted at $0.4 \mathrm{~mL} / \mathrm{min}$ with a $\mathrm{RP}$ gradient method: $0.0-1.0 \mathrm{~min}$ at $5 \% \mathrm{~B} ; 1.0-12.0 \mathrm{~min}$ to $95 \% \mathrm{~B} ; 12.0-14.0 \mathrm{~min}$ at $95 \% \mathrm{~B} ; 14.1-$ 16.0 min at $5 \% \mathrm{~B}$, with 4 minutes of post time equilibration after each run. Detection was done on the ESI-TOF MS in the negative ionization mode at mass range of 100-3000 $\mathrm{m} / \mathrm{z}$. Figure 81 shows the overlaid chromatograms of five reference standards injections for: catechin (CATE), epicatechin (EPI), quercetin 3-O-glucoside (Q3G), kaempferol 3O-glucoside $(\mathrm{K} 3 \mathrm{G})$, and quercetin. Longest retention and best overall separation were achieved by the C20 column; however, catechin and epicatechin were resolved most efficiently by the TOCO phase. In summary, all three columns are fully operational for the analysis of flavonoids.

All five skin extracts seem to contain a substantial amount of all reference analytes (Figure 82, 83) except for quercetin (Figure 84). The following figures were generated by MAVEN software and displays relative abundance of the analytes of interest in five grape samples eluted by three columns. As indicated as a bar graph on the right-hand side of the figure, all three compounds were generally more abundant in the RSTGs - SR and $\mathrm{C} 33$. 


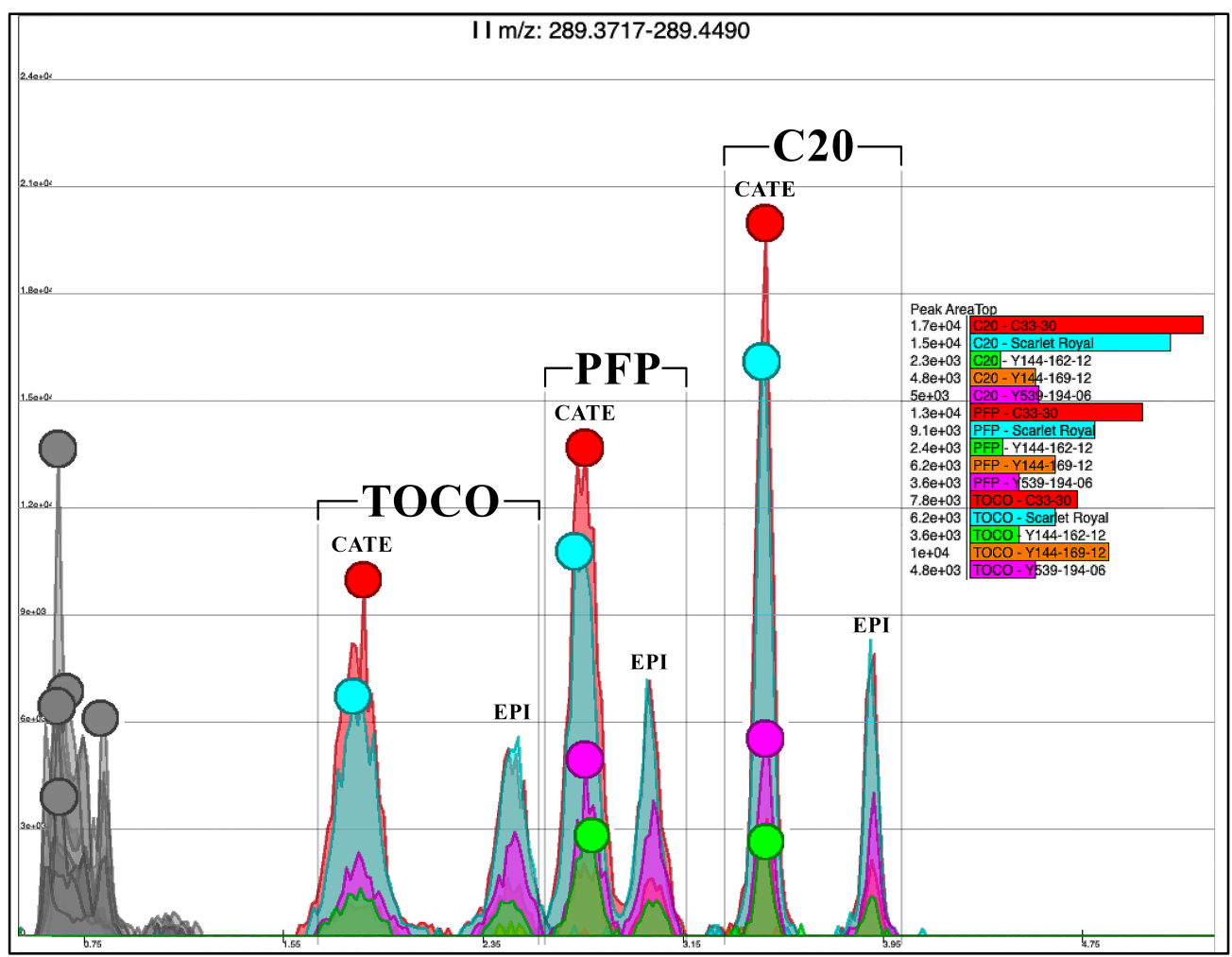

Figure 82. Catechin and Epicatechin Detected in Five Grape Cultivars.

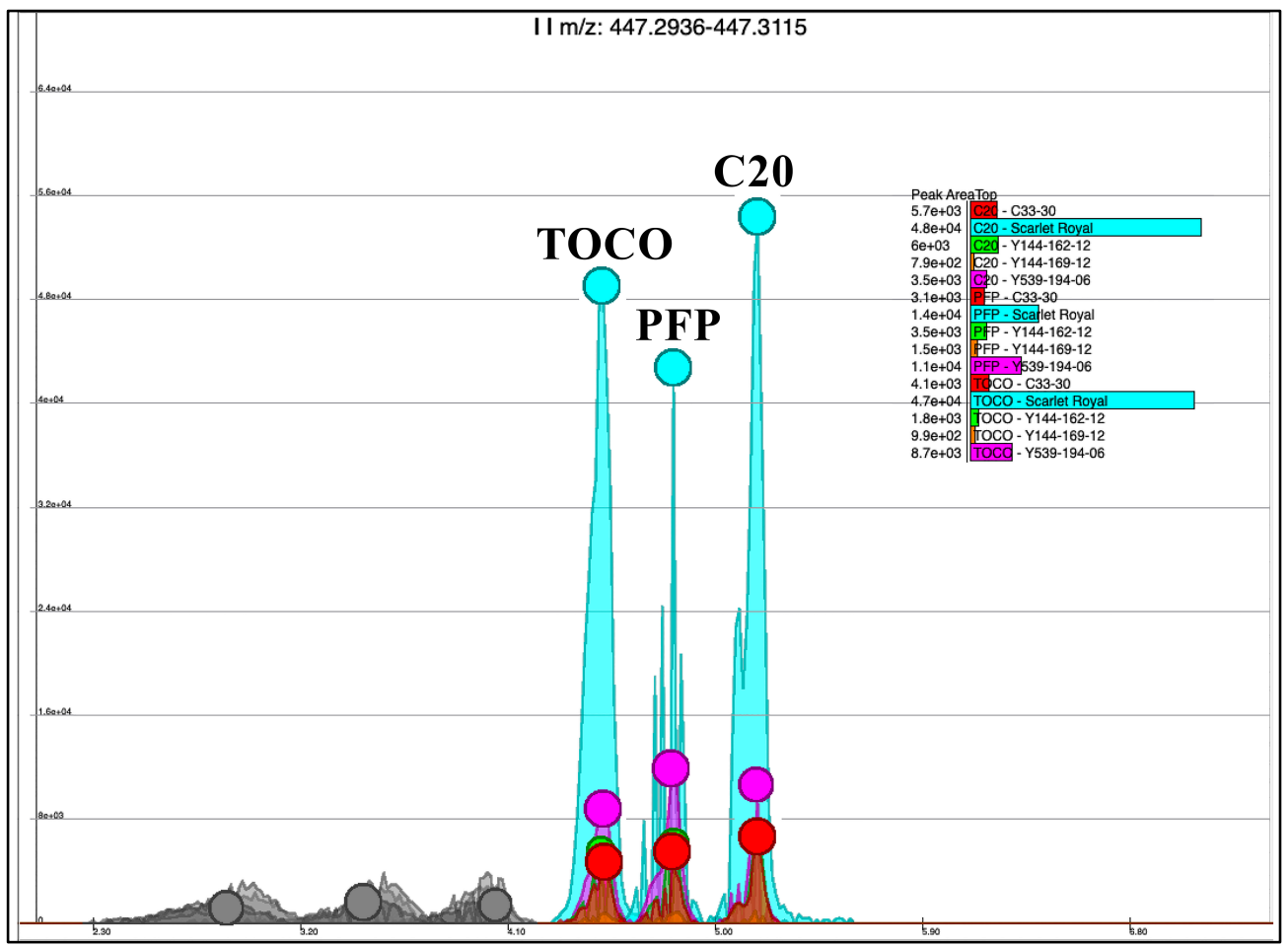

Figure 83. K3G Detected in Five Grape Cultivars. 


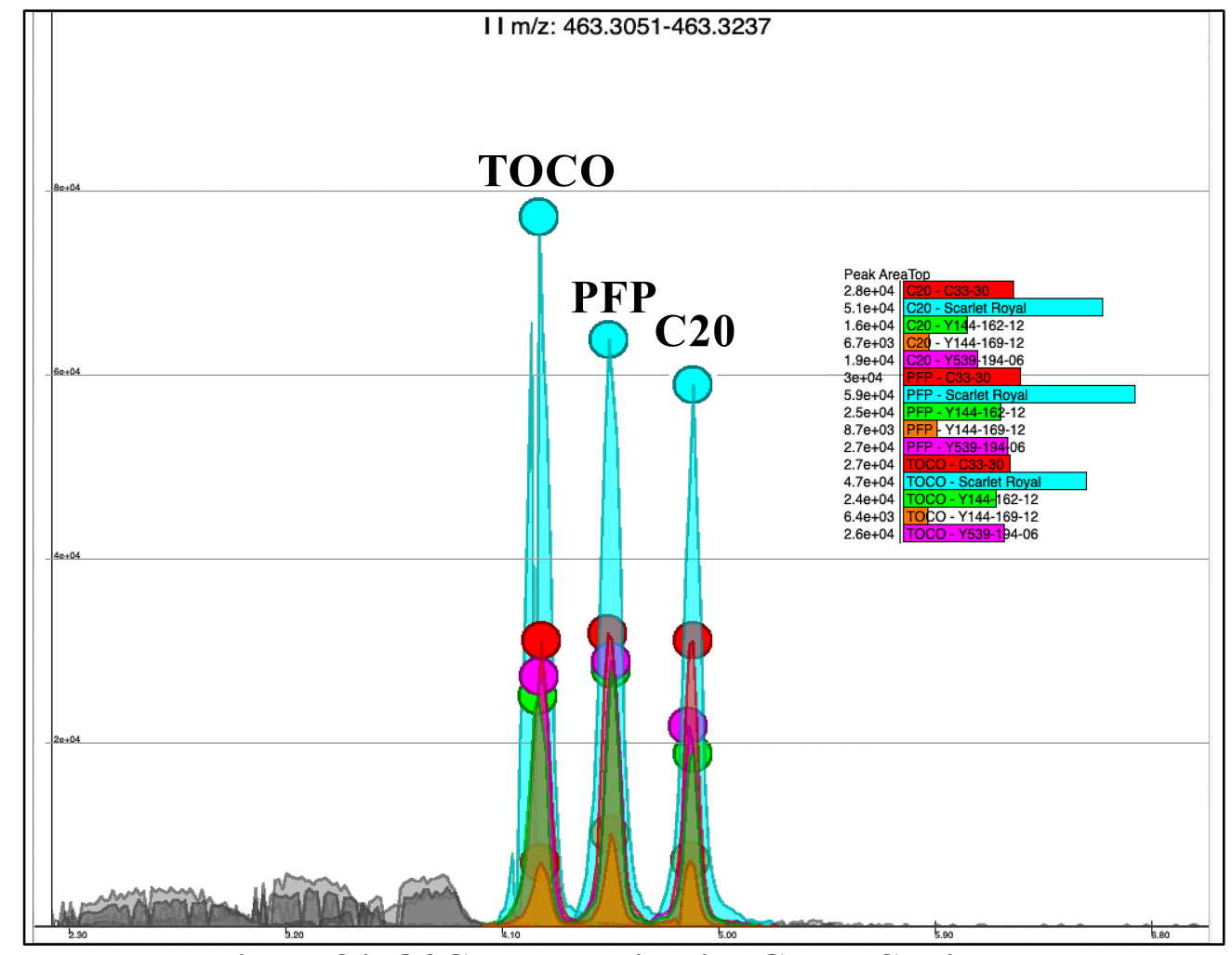

Figure 84. Q3G Detected in Five Grape Cultivars.

It is also apparent that other three Y-series grapes (\#162, \#169, and Y539) have a similar trend for \#162 and Y539; however, \#169 grape exhibits a unique metabolic profile from the other two. For example, \#169 has a relatively high abundance for catechin and epicatechin but the Q3G abundance is lower than \#162 and Y539. The absence of quercetin intermediate was consistent across all cultivars and possibly due to consumption during $\mathrm{Q} 3 \mathrm{G}$ production among other metabolic products within the flavonoid and flavone/flavonol biosynthetic pathways (Figure 85). 


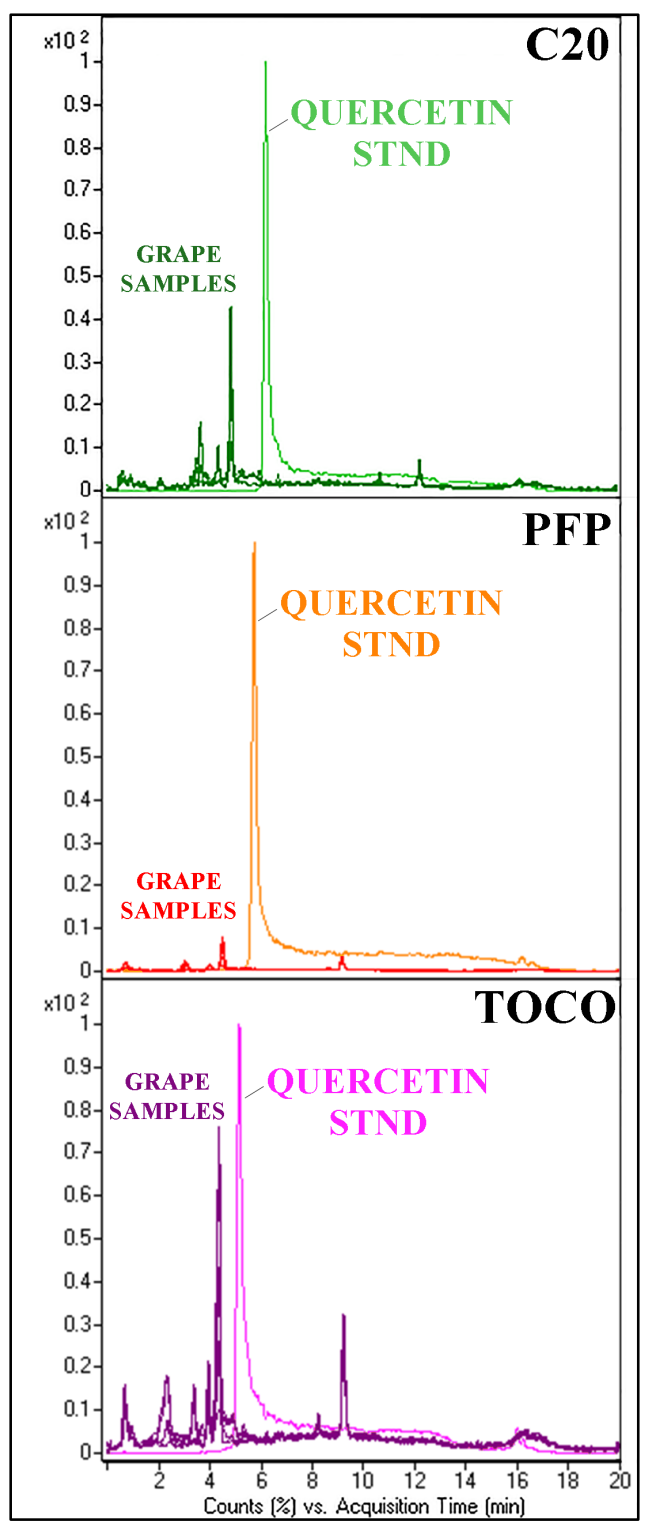

Figure 85. EIC Overlay of Quercetin Standard and Five Grape Sample Injections.

Differences in metabolic profiles across the grape cultivars are essentially phenotypic variations caused by the distinct genotypes of individual cultivar and/or different extents of gene expression affected by numerous environmental factors. Metabolomics is a powerful tool for the identification of candidate enzymes or genes that may have caused the phenotypic differences observed in the grape samples. For instance, one of the ways to explain the increased abundance of CATE/EPI in SR and C33 grapes are the increased 
metabolic flux from dihydroquercetin (DHQ) to leucocyanidin (LCY) in the flavonoid biosynthetic pathway (Figure 86).

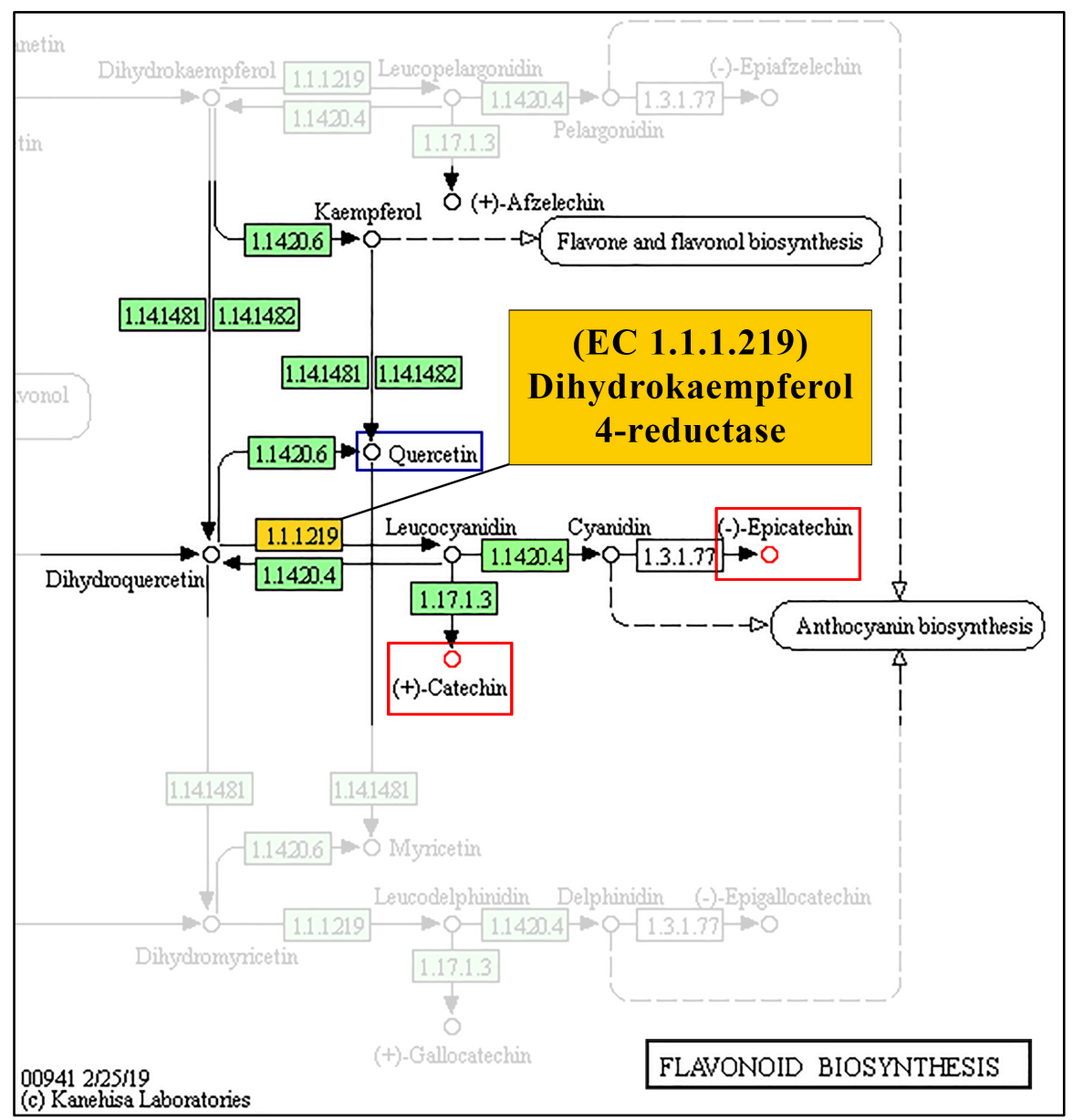

Figure 86. Flavonoid Biosynthetic Pathway.

Catalysis of this reaction is controlled by dihydroflavonol 4-reductase (DFR), which is known to be the rate-limiting enzyme in anthocyanin biosynthetic pathway in several plant species $[87,88]$. This is one explanation to the increased production of both metabolites since LCY is a precursor to CATE and an essential intermediate for the synthesis of EPI. Increase in both $\mathrm{Q} 3 \mathrm{G} / \mathrm{K} 3 \mathrm{G}$ concentration for $\mathrm{SR}$ is potentially related to the activity of flavonol 3-O-glucosyltransferase, which is responsible for glycosylation 
of kaempferol and quercetin in the flavone and flavonol biosynthetic pathway (Figure 87) $[89,90]$.

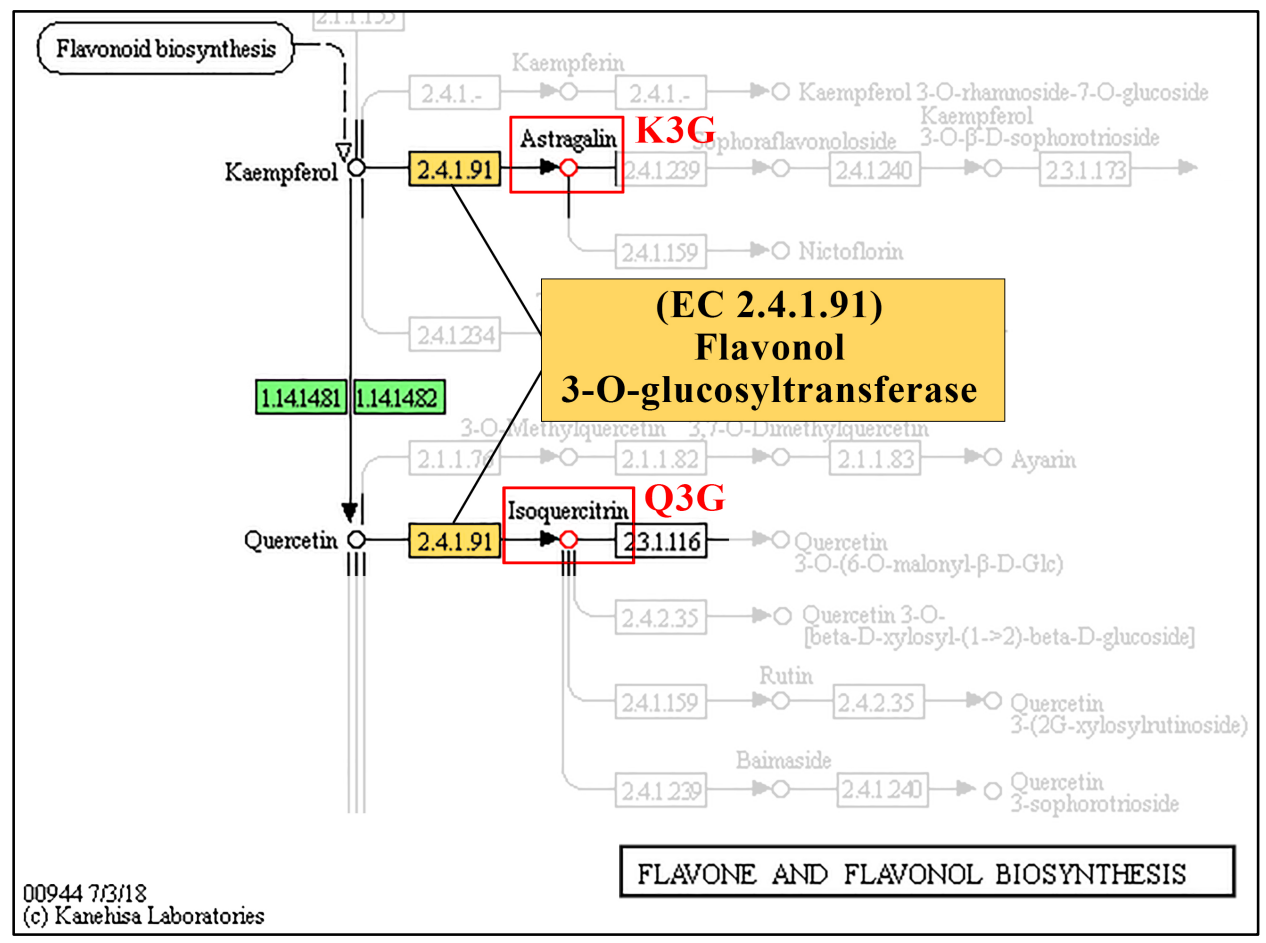

Figure 87. Flavone and Flavonol Biosynthetic Pathway.

However, the selective increase of Q3G in C33 is more likely an outcome of increased kaempferol $\rightarrow$ quercetin flux, which is regulated by other enzymes (EC 1.14.14.81-82).

The comprehensive metabolome abundance profiles are shown in Figure 88 as a clustered heatmap representation. Each colored line represents a peak group (based on the unique combination of $\mathrm{M} / \mathrm{Z} \& \mathrm{tR}$ ) with red indicating high and blue as low abundance of analyte ions (the natural log of ion counts). Clustered abundance profile indicates C33 and SR under the same cluster and Y-series grapes under their own category. Based on this analysis, \#162 grape has potentially inherited more metabolic traits from its father Y539 more than \#169 cultivar did. In order to better understand the chemical 
components that genetically distinguishes grape cultivars, a set of grape samples from one cluster was compared against another by using scatter plots.

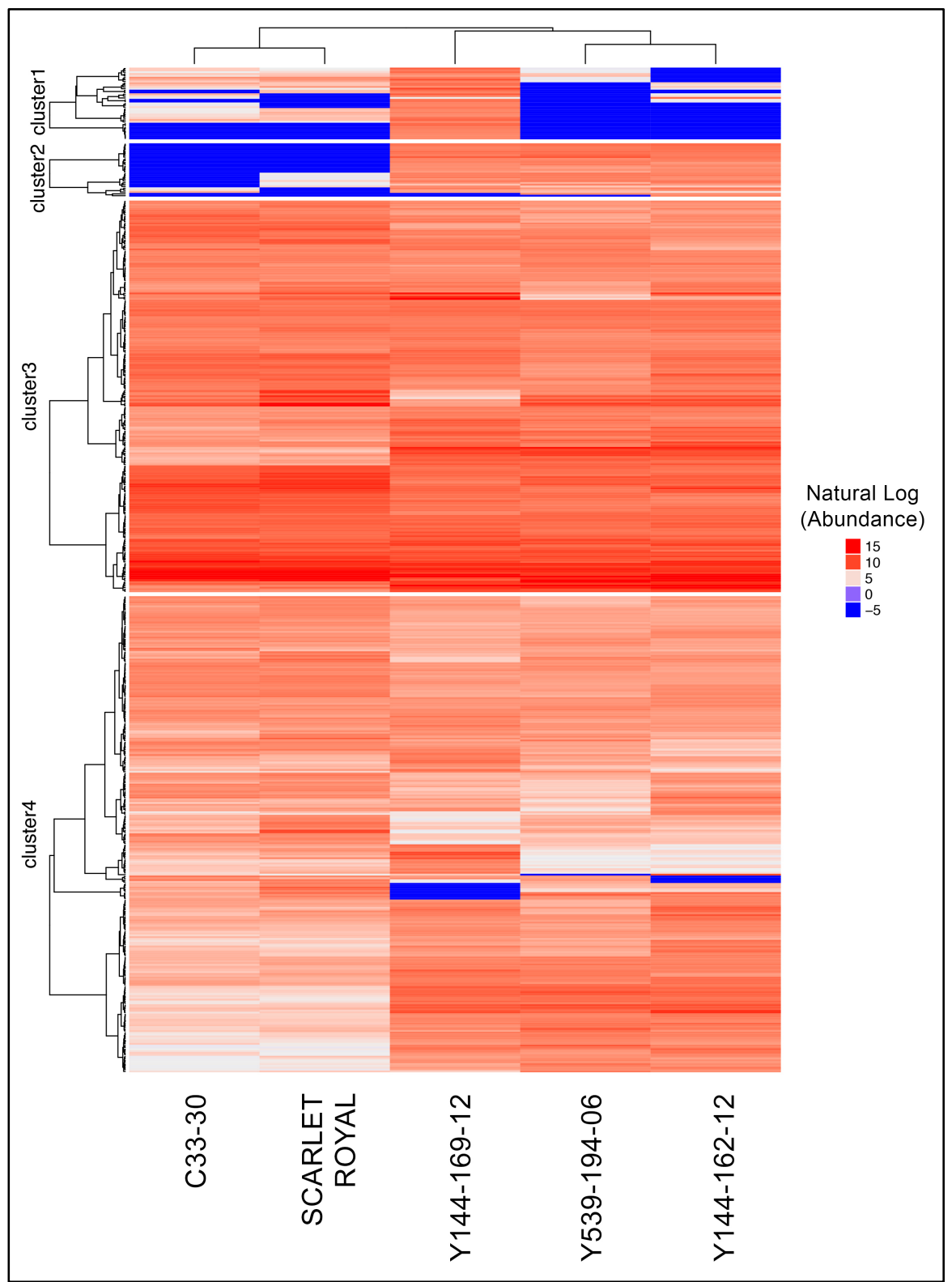

Figure 88. Heatmap Representation of Relative Metabolite Abundance.

Figure 89 shows scatter plots comparing a set of two red grapes - (Set 1- C33/SR) against the three purple grapes (Set 2 - Y539/\#162/\#169). X-axes represent the metabolite abundance observed in Set 1 samples and the Y-axes represents the Set 2. Each red-colored 
dot represents a peak group where Set 1 has a higher relative peak area than Set 2 (Group A), and vice versa for blue dots (Group B). In this diagram, only the analyte peak groups with statistically significant differences $(\mathrm{p}<0.05)$ between the two sample sets are displayed. All three peaks under the group A are highly abundant in the two RTSGs (496, $914,264 \mathrm{~m} / \mathrm{z}$ ), while B shows the analyte peaks more particularly present in the Y-series grapes $(1091,492,315 \mathrm{~m} / \mathrm{z})$. According to the Scripps METLIN metabolite database, 492 $\mathrm{m} / \mathrm{z}$ matches the $\mathrm{m} / \mathrm{z}$ value of $[\mathrm{M}-\mathrm{H}]^{-}$adduct of malvidin 3-O-glucoside (M3G) which is one of the anthocyanins commonly present in purple grapes like the three $\mathrm{Y}$-series grapes. The structure of $\mathrm{M} 3 \mathrm{G}$ is analogous to $\mathrm{Q} 3 \mathrm{G} / \mathrm{K} 3 \mathrm{G}$, which explains why the retention time is similar to other 3-glucoside of flavonoids. There were matches for two more $\mathrm{m} / \mathrm{z}$ values: $[\mathrm{M}+\mathrm{Cl}]^{-}$adduct of cyanidin 3-O-(6"-acetyl-arabinoside) at $496 \mathrm{~m} / \mathrm{z}$, and [M-H] $]^{-}$adduct for leucodelphinidin 4-O-[2,4-bisgalloyl-6-(3-galloylgalloyl)-beta-D-glucopyranoside] at $1091 \mathrm{~m} / \mathrm{z}$.

Figure 90 displays the results of comparison between Y539/\#162 (Set 1) and \#169 (Set 2) cultivars. Set 1 showed higher abundance for two peak groups (group C) at 178, and $432 \mathrm{~m} / \mathrm{z}$. Again, the identity of $432 \mathrm{~m} / \mathrm{z}$ peak can be a flavonoid glucoside based on its similar retention time to $\mathrm{Q} 3 \mathrm{G} / \mathrm{K} 3 \mathrm{G}$. In fact, the METLIN database indicated the potential match for $432 \mathrm{~m} / \mathrm{z}$ as $[\mathrm{M}-\mathrm{H}]^{-}$adduct of flavonoid glycosides (e.g., isopeonidin- x 2, peonidin- $x$ 2, petunidin-, cyanidin-, luteolinidin-, pelargonidin-). In the meantime, two intense peak groups were observed for \#169 (group D): potential [M-H] ${ }^{-}$adduct of cyanidin 3-malonylglucoside $(534 \mathrm{~m} / \mathrm{z})$ at $6.02 \mathrm{mins}$ and $[\mathrm{M}+\mathrm{Cl}]^{-}$adduct of petunin $(676 \mathrm{~m} / \mathrm{z})$ at 7.27 mins. 


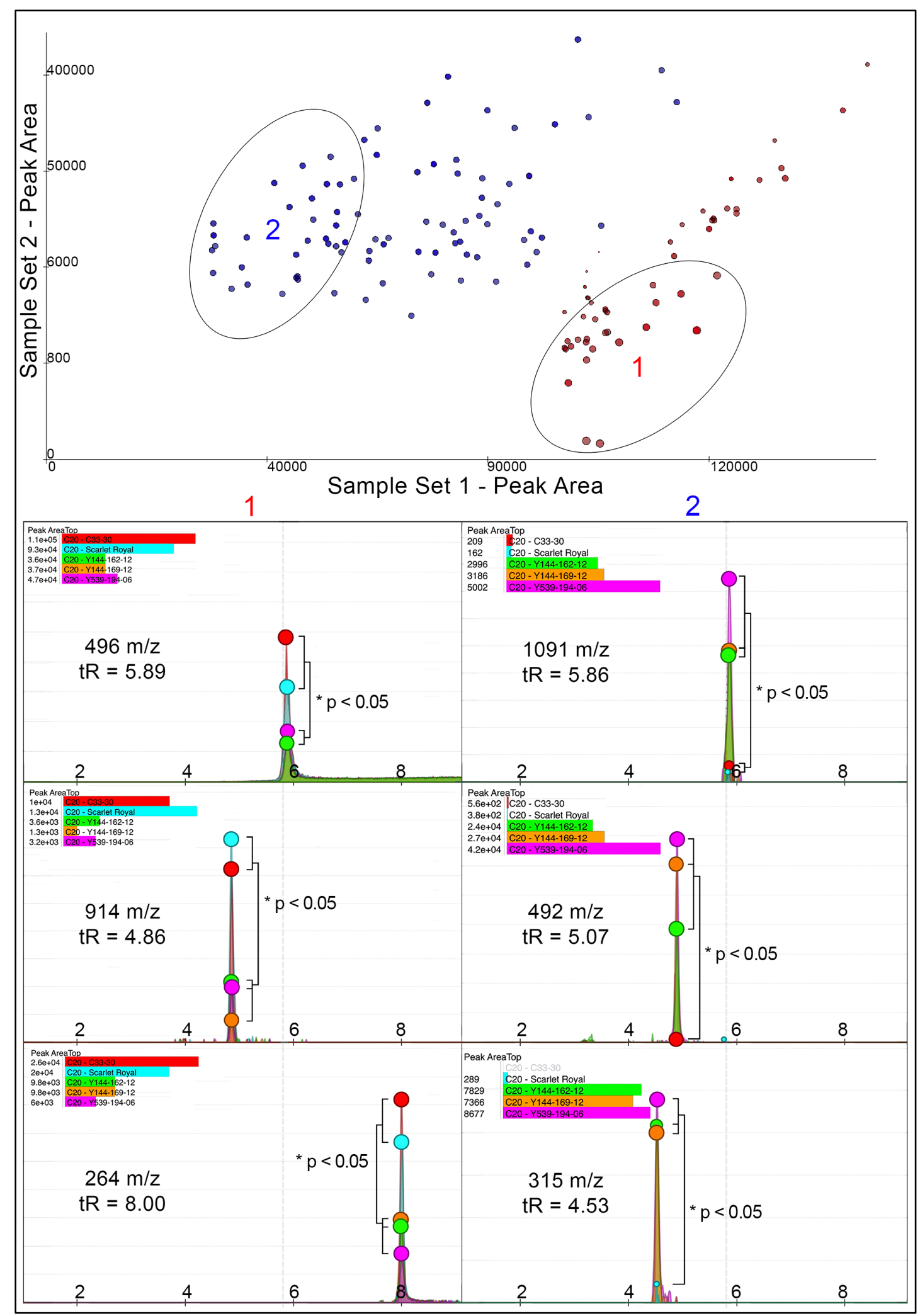

Figure 89. Metabolome Abundance Differences Between the Two Types of Grapes. 


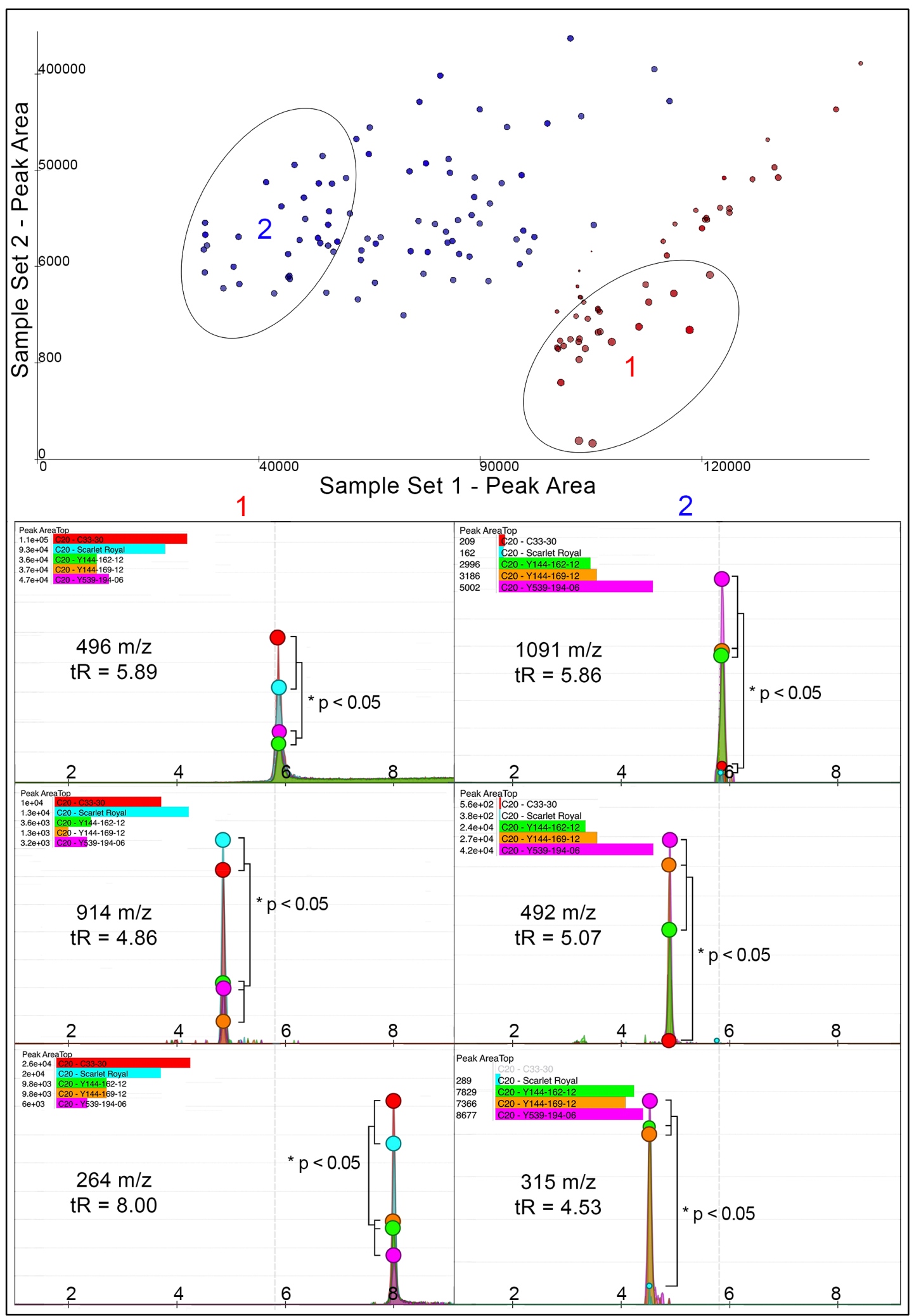

Figure 90. Metabolome Abundance Differences Between Y539/\#162 vs. \#169. 


\subsection{Proton Back-exchange Reduction by ANP-coupled HDX-MS}

As discussed in Section 2.3.6., ANP chromatography can theoretically decrease the

D-H back-exchange (BE) during elution by reducing the amount of protic solvent usage.

Figure 91 shows the mass distribution of $\mathrm{D}_{2} \mathrm{O}$-reconstituted enkephalin peptide $\left(\mathrm{D}_{2} \mathrm{O}\right.$ -

EKP) eluted at 100-10\% B isocratic conditions, which may support this hypothesis.

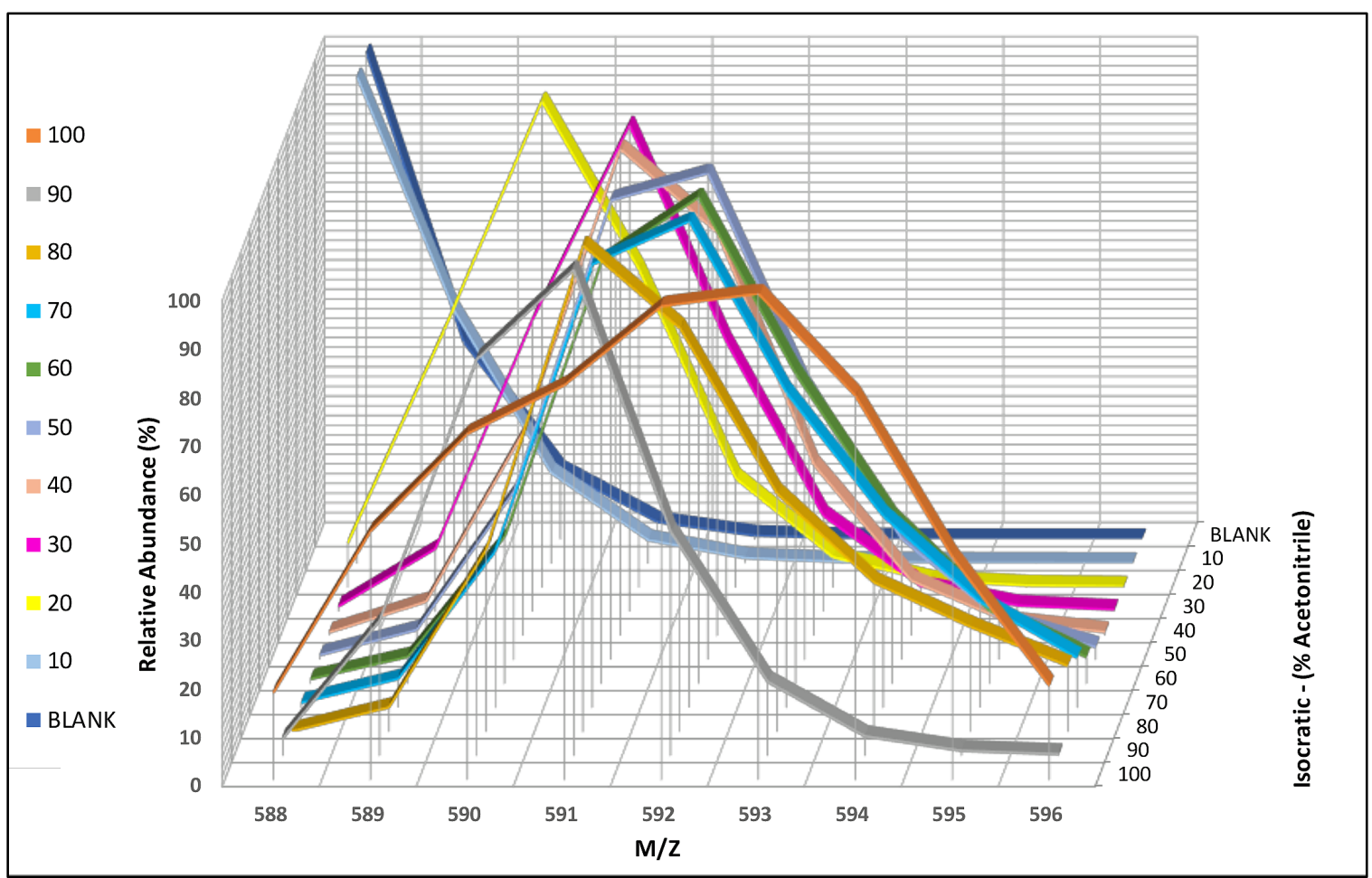

Figure 91. Isotope Mass Distribution of $\mathrm{D}_{2} \mathrm{O}$-EKP at Various Elution Conditions.

Since the EKP peptide virtually makes no contact with protiated solvent water at $100 \% \mathrm{~B}$, the corresponding mass spectrum shows high abundance of fully deuterated EKP $(592 \mathrm{~m} / \mathrm{z})$. Moreover, high ion abundance at $593-596 \mathrm{~m} / \mathrm{z}$ indicates that peptides are possibly deuterated at non-amide positions as well (in dark orange). The spectrum of EKP eventually completely shifts towards the lower non-deuterated mass $(588 \mathrm{~m} / \mathrm{z})$ as water composition increases. At $10 \% \mathrm{~B}$, mass profile of $\mathrm{D}_{2} \mathrm{O}-\mathrm{EKP}$ is essentially identical 
to the mass of blank, which is an EKP reconstitute in $\mathrm{H}_{2} \mathrm{O}\left(\mathrm{H}_{2} \mathrm{O}-\mathrm{EKP}\right.$; in dark blue). This trend seems to be consistent except at $90 \%$ B where the retention time of the peptide drastically increases; consequently, $\mathrm{D}_{2} \mathrm{O}$-EKP peptides are exposed to protons for a longer period (in gray).

In Figure 92, $\mathrm{D}_{2} \mathrm{O}-\mathrm{EKP}$ elution is demonstrated by using ANP/RP gradient methods. Realistically, the isocratic elution of the peptides is not advisable since it tends to produce broader peak shape. Here, analyte retention by ANP gradient (green) is achieved on a C20 RP column at comparable levels to RP gradient elution (red) performed on the same column. The mass spectrum shown on the right indicates lower BE for the ANP gradient compared to the RP gradient. Although, reduced time of solvent exposure also likely contributes in lowering the BE.

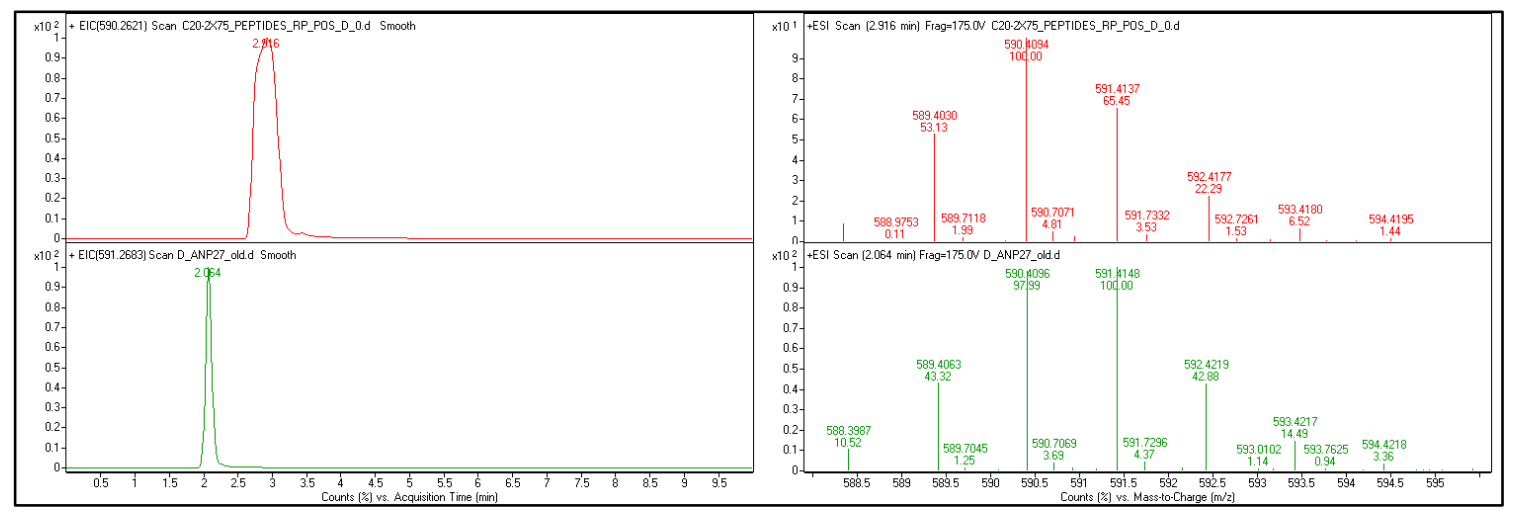

Figure 92. ANP and RP Elution of $\mathrm{D}_{2} \mathrm{O}$-EKP at Normalized $\% \mathrm{H}_{2} \mathrm{O}$ Composition.

Based on the calculated average masses of $\mathrm{D}_{2} \mathrm{O}$-EKP peptides, the $\mathrm{BE}$ profile of the ANP gradient method is equivalent to about 20-30\% B isocratic conditions (Table 19). However, the retention time observed for the ANP gradient is relatively longer than the two isocratic conditions used. The demonstrated ANP/RP gradient had a normalized \% 
$\mathrm{H}_{2} \mathrm{O}$ composition of about $54 \%$; hence peptide retention observed from the ANP gradient is not as strong as the RP gradient.

Table 19. Isotope Mass Distribution of $D_{2} O$-EKP Peptide at All Tested Conditions.

\begin{tabular}{|c|c|c|c|c|c|c|c|c|c|c|c|c|c|}
\hline $\mathbf{M} / \mathbf{Z}$ & $\underbrace{00^{103}}$ & $90^{00^{3}}$ & $8^{80^{10}}$ & $0^{0,0^{30}}$ & $6^{10^{30}}$ & $50^{00^{3}}$ & $x^{00^{30}}$ & $3^{00^{108}}$ & $2^{00^{30}}$ & $8^{108}$ & $5^{+5}$ & $E^{s}$ & \& \\
\hline 588 & & & & & & & & & & & & & \\
\hline 589 & & & & & & & & & & & & & \\
\hline 590 & & & & & & & & & & & & & \\
\hline 591 & & & & & & & & & & & & & \\
\hline 592 & & & & & & & & & & & & & \\
\hline 593 & & & & & & & & & & & & & \\
\hline 594 & & & & & & & & & & & & & \\
\hline 595 & & & & & & & & & & & & & \\
\hline 596 & & & & & & & & & & & & & \\
\hline $\operatorname{Avg}(\mathbf{M} / \mathrm{Z})$ & 592.0 & \begin{tabular}{|l|}
590.8 \\
\end{tabular} & 592.1 & 592.2 & 592.1 & 591.9 & 591.5 & 591.1 & 590.3 & 588.6 & 588.5 & 590.6 & 590.3 \\
\hline tR (mins) & 0.57 & 1.61 & 0.97 & 0.79 & 0.73 & 0.78 & 0.79 & 0.99 & 2.05 & $>20$ & - & 2.06 & 2.92 \\
\hline \#D & 3.5 & 2.3 & 3.6 & 3.7 & 3.6 & 3.3 & 3.0 & 2.6 & 1.7 & 0.1 & 0.0 & 2.0 & 1.7 \\
\hline
\end{tabular}

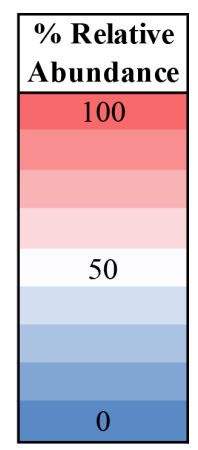

While the ANP gradient with shorter retention time clearly shows lower BE than the RP gradient, the difference in average masses were much less significant than anticipated ( 590.6 vs. $590.3 \mathrm{~m} / \mathrm{z}$ ). Essentially, the extent of BE may not be solely controlled by the amount of water consumption or the length of solvent exposure. Instead, the results suggest that $\mathrm{BE}$ is more critically affected by the mobile phase composition the peptides are exposed to (e.g., aqueous/organic ratio).

In order to effectively assess the solvent composition effects, the ANP and RP gradient methods must be designed to produce an identical retention time to normalize the time of solvent exposure. Figure 93 shows the retention time of angiotensin-II (A-II; $523 \mathrm{~m} / \mathrm{z}$ at $[\mathrm{M}+2 \mathrm{H}]^{2+}$ ) peptide under RP (red) and ANP (green) gradient conditions. 


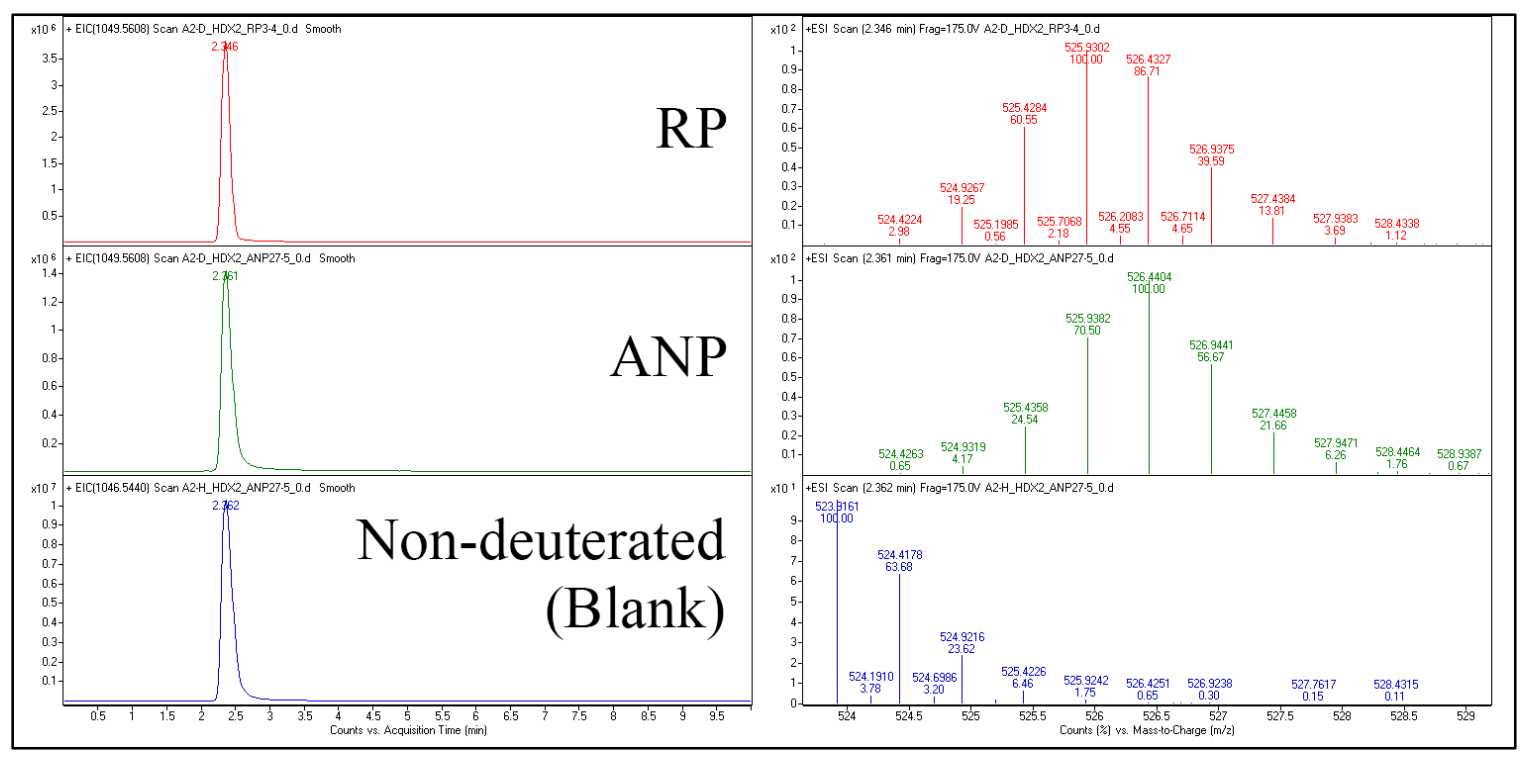

Figure 93. ANP and RP Elution of A-II at Normalized Retention Time.

Table 20. A-II Peptide Mass Profile at Normalized Solvent Exposure Time.

\begin{tabular}{|c|c|c|c|}
\hline $\mathbf{M} / \mathbf{Z}$ & Blank & ANP & RP \\
\hline 523.9 & 100.0 & 0.0 & 0.0 \\
\hline 524.4 & 63.7 & 0.6 & 2.9 \\
\hline 524.9 & 23.6 & 4.2 & 19.1 \\
\hline 525.4 & 6.5 & 24.6 & 59.8 \\
\hline 525.9 & 1.8 & 71.2 & 100.0 \\
\hline 526.4 & 0.7 & 100.0 & 86.7 \\
\hline 526.9 & 0.3 & 56.7 & 39.6 \\
\hline 527.4 & 0.0 & 21.9 & 13.5 \\
\hline 527.9 & 0.0 & 6.3 & 3.7 \\
\hline 528.4 & 0.0 & 1.7 & 1.1 \\
\hline Avg. (M/Z) & 524.1 & 526.3 & 526.0 \\
\hline tR (mins) & - & 2.36 & 2.35 \\
\hline \%-H2O & - & 49.7 & 60.6 \\
\hline mL-H2O & - & 0.47 & 0.57 \\
\hline \#D & - & 4.3 & 3.7 \\
\hline
\end{tabular}

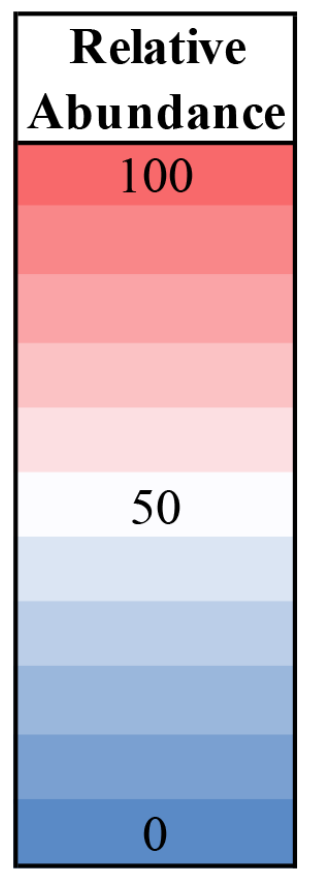


It is apparent that A-II peptide eluted at the same retention time does not produce identical mass profiles (Table 20). The ANP gradient shows mass spectrum slightly shifted towards higher $\mathrm{m} / \mathrm{z}$ values and the most abundant peptide ions were observed at $526.4 \mathrm{~m} / \mathrm{z}$ (equivalent to peptide mass deuterated at five locations); meanwhile, the RP gradient provides the highest mass abundance of $525.9 \mathrm{~m} / \mathrm{z}$ ions (equivalent to four deuterations). About $0.1 \mathrm{~mL}$ of total $\mathrm{H}_{2} \mathrm{O}$ volume difference (roughly $10 \%$ difference in $\mathrm{H}_{2} \mathrm{O}$ composition) has increased the average mass by more than half of a deuterium mass. Based on 95\% confidence statistical significance test, the difference in BE profile between the two modes of separation at tested conditions are statistically significant $(\mathrm{P}<$ 0.05 ; Table 21).

Table 21. Statistical Significance Test for BE Reduction by ANP Gradient.

\begin{tabular}{|c|c|}
\hline \multicolumn{2}{|c|}{$\Delta \# D$} \\
\hline \hline Mean & 0.622 \\
\hline SD & 0.023 \\
\hline N & 6 \\
\hline Level of Probability & $5 \%$ \\
\hline Std Error & 0.01 \\
\hline Actual T-Value & 67.67 \\
\hline Critical T-Value & 2.57 \\
\hline "P" & $1.33 E-08$ \\
\hline \hline Significance: & Significant \\
\hline
\end{tabular}

The same chromatographic condition was tested on a PFP column which possesses weaker RP characteristics than the C20 column; hence ANP retention is stronger even in 
the same mobile phase composition. The Figure 94 indicates that ANP has higher relative peptide mass than the RP gradient, despite longer retention time achieved.

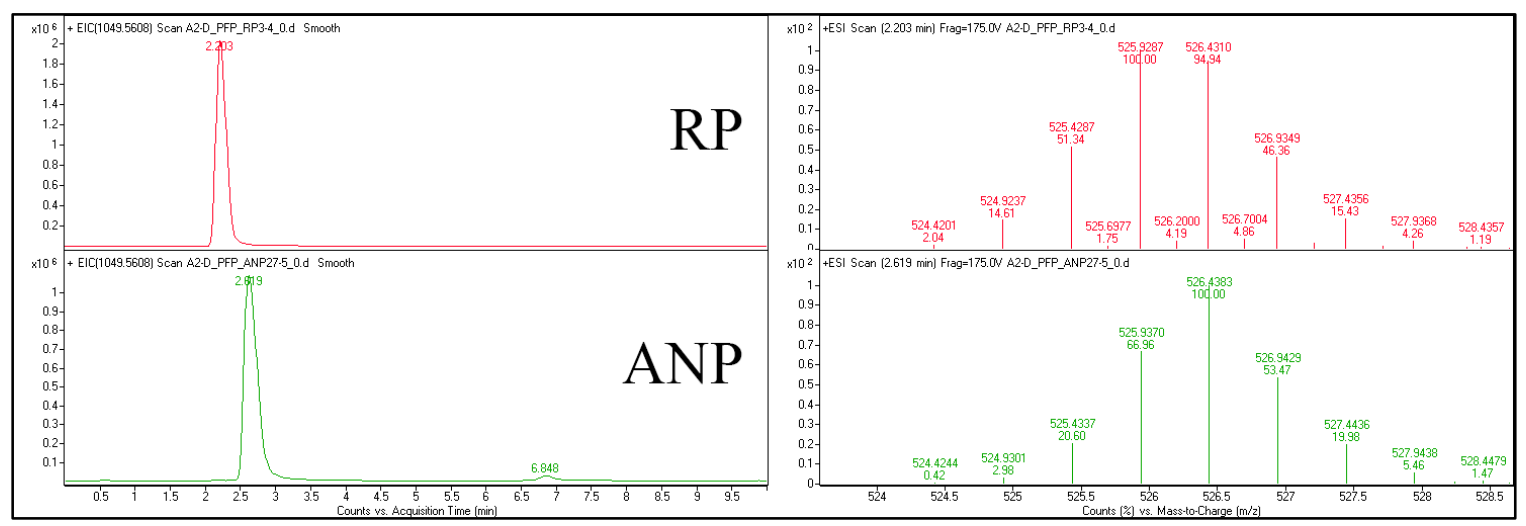

Figure 94. A-II Peptide Elution with ANP/RP Gradient Using PFP Column.

Table 22. A-II Peptide Mass Profile at Normalized Aqueous Solvent Volume.

\begin{tabular}{|c|c|c|c|}
\hline $\mathbf{M} / \mathbf{Z}$ & Blank & ANP & RP \\
\hline 523.9 & 100.0 & 0.0 & 0.0 \\
\hline 524.4 & 63.7 & 0.4 & 2.0 \\
\hline 524.9 & 23.6 & 3.0 & 14.6 \\
\hline 525.4 & 6.5 & 20.6 & 51.3 \\
\hline 525.9 & 1.8 & 67.0 & 100.0 \\
\hline 526.4 & 0.7 & 100.0 & 94.9 \\
\hline 526.9 & 0.3 & 53.5 & 46.4 \\
\hline 527.4 & 0.0 & 20.0 & 15.4 \\
\hline 527.9 & 0.0 & 5.5 & 4.3 \\
\hline 528.4 & 0.0 & 1.5 & 1.2 \\
\hline Avg. (M/Z) & 524.1 & 526.3 & 526.1 \\
\hline tR (mins) & - & 2.62 & 2.20 \\
\hline \%-H2O & - & 50.7 & 61.8 \\
\hline mL-H2O & - & 0.53 & 0.54 \\
\hline \#D & - & 4.3 & 3.8 \\
\hline
\end{tabular}

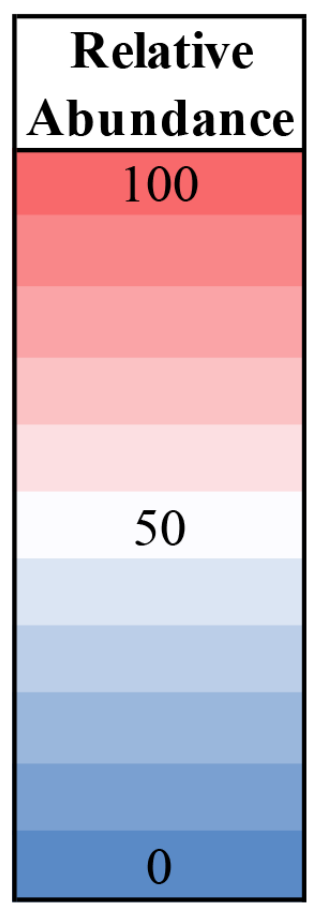


Overall, the ANP gradient consumes nearly as much water volume as the RP gradient does: $0.53 \mathrm{~mL}$ and $0.54 \mathrm{~mL}$, respectively (Table 22). Due to the nature of ANP chromatography, retention of polar analytes can be achieved with relatively low amount of water. In fact, ANP retention on a PFP column extended the retention time by $\sim 0.3$ minutes compared to $\mathrm{C} 20$ but with no significant change in mass profile. Potentially, BE by ANP-HDX-MS could be further enhanced by implementing additional equipment for BE minimization such as: short, small particle-sized column coupled to the UHPLC and/or refrigerated-LC system [91].

\subsection{Selectivity Test}

Lastly, analyte selectivity of each column was assessed by observing the ANP/RP retention behavior of several compound groups. For the RP gradient, initial condition was set to $10 \% \mathrm{~B}$ from $0.0-0.5 \mathrm{~min} ; 0.5-3.0 \mathrm{~min}$ to $80 \% \mathrm{~B} ; 3.0-4.0 \mathrm{~min}$ at $80 \% \mathrm{~B}$; 4.0 $5.0 \mathrm{~min}$ at $10 \% \mathrm{~B}$; and $5.0-10.0 \mathrm{~min}$ at $10 \% \mathrm{~B}$. For the ANP gradient: $0.0-0.5 \mathrm{~min}$ at $90 \% \mathrm{~B} ; 0.5-3.0 \mathrm{~min}$ to $30 \% \mathrm{~B} ; 3.0-4.0 \mathrm{~min}$ at $30 \% \mathrm{~B} ; 4.0-5.0 \mathrm{~min}$ at $90 \% \mathrm{~B}$; and $5.0-$ $10.0 \mathrm{~min}$ at $90 \%$ B. Elution was done at $0.4 \mathrm{~mL} / \mathrm{min}$ flow rate using $\mathrm{DI} \mathrm{H}_{2} \mathrm{O}+0.1 \%$ formic acid (A) and acetonitrile $+0.1 \% \%$ formic acid as mobile phase.

Figure 95 shows the retention trends of eleven steroids on three columns. As expected from typical RP retention, molecules with higher counts of polar functional

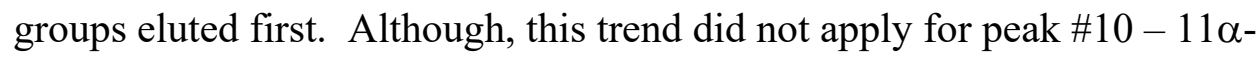
acetoxyprogesterone with the fourth lowest calculated partition coefficient (CLogP) among eleven steroids. Unique selectivity from each column was also observed to some extent for PFP at peak $\# 2$ and $\# 7$, and TOCO for peak \#5, \#7, and \#9 to a lesser extent. 
Red-colored numbering indicates the retention order change compared to the elution order of $\mathrm{C} 20$. The TOCO phase seems to well-retain steroids consisting of alkyne groups (peak \#5, 7, and 9).

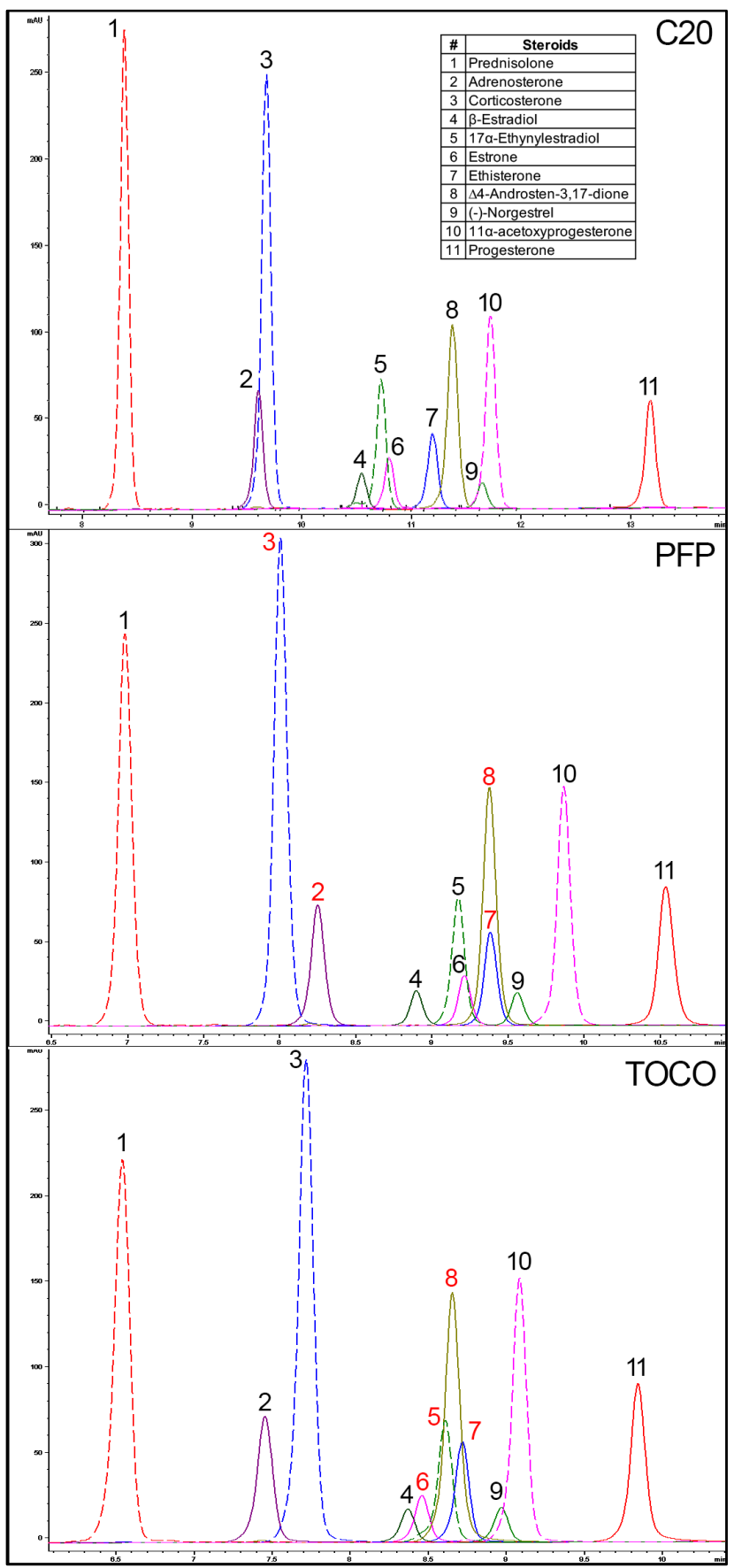

Figure 95. RP Retention and Selectivity Profile of Eleven Steroids. 
The next group of analytes are called "nootropics", commonly known as "smart drugs" or "cognitive enhancers". Two types of nootropics observed were: six amphipathic racetams (2-pyrrolidone derivatives) and two hydrophilic cholinergic molecules.

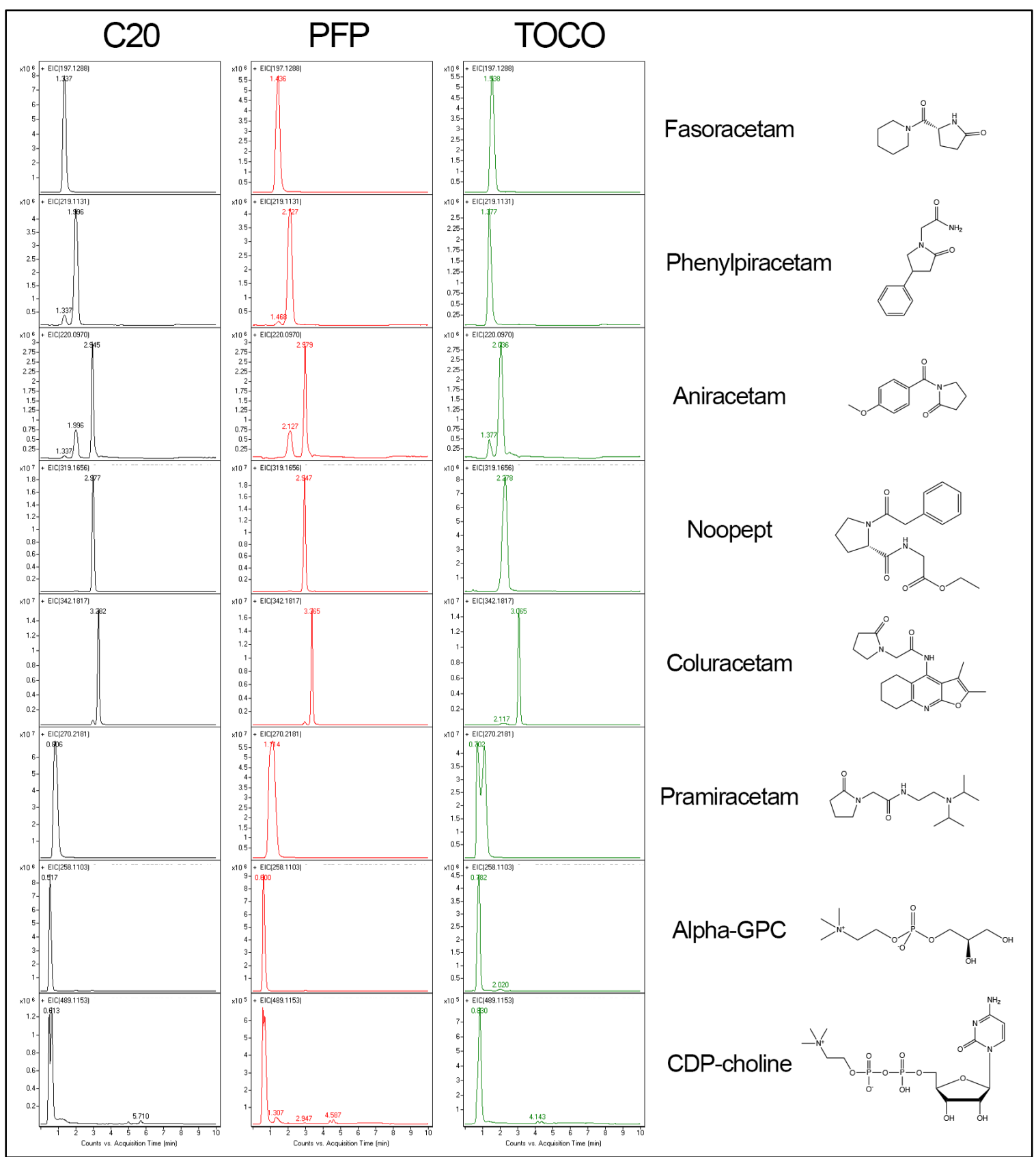

Figure 96. RP Retention and Selectivity Profile of Eight Nootropic Drugs. 


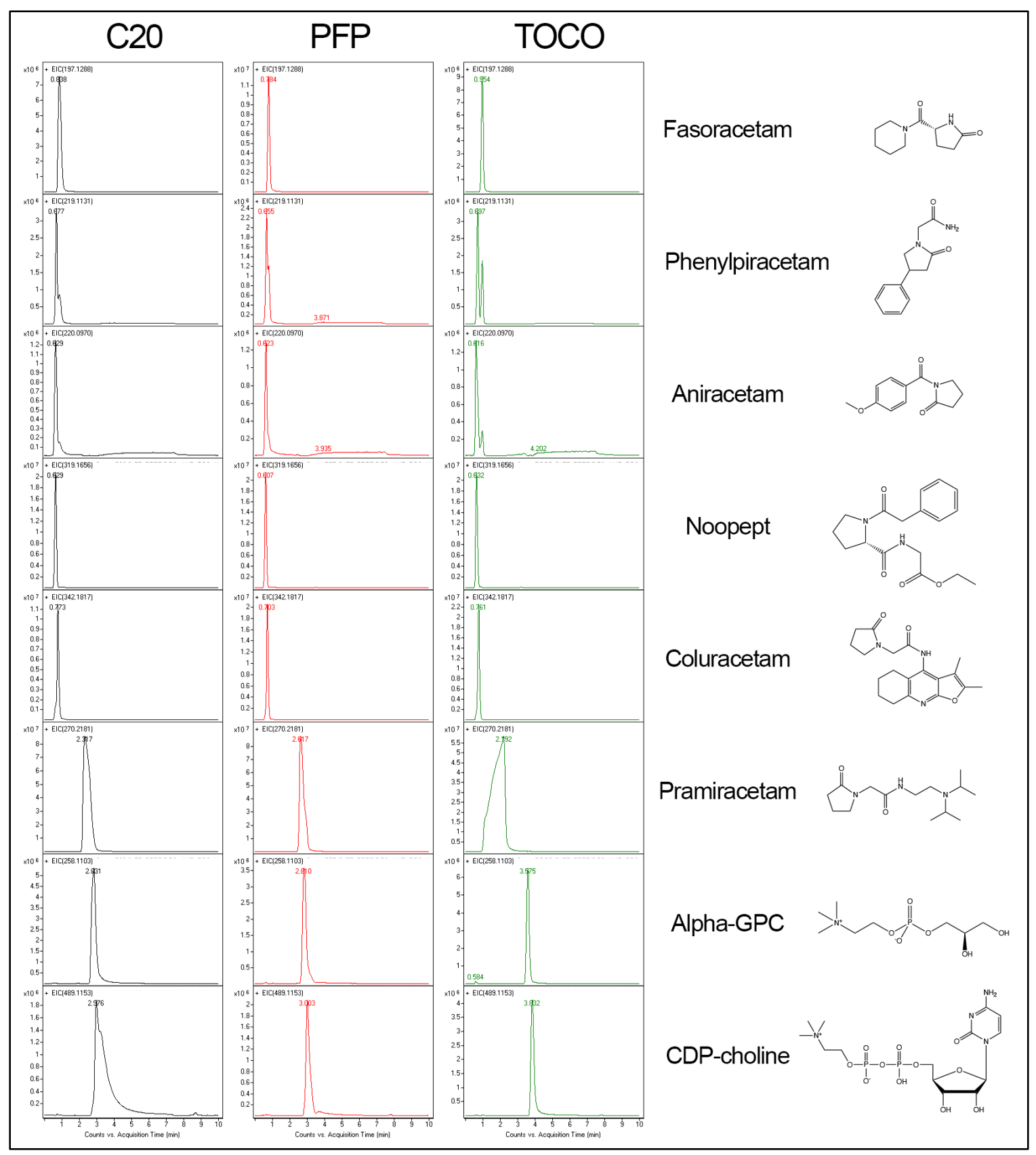

Figure 97. ANP Retention and Selectivity Profile of Eight Nootropic Drugs.

Figure 96 shows the retention profile of racetams under RP conditions. Pramiracetam was the only racetam molecule unretained under RP conditions along with relatively polar alpha-GPC and CDP-choline. Other racetam molecules were retained and wellseparated except for aniracetam and noopept. At the elution condition used, TOCO 
seems to be the only phase able to distinguish and separate the two molecules by nearly 0.25 mins. Fasoracetam also seem to favor interaction with the TOCO phase and thus retained the longest among the three columns.

Figure 97 shows the ANP retention of molecules previously unretained by RP mode. The peak distortion of pramiracetam is potentially due to overloading and slight peak tailing observed for cholinergic molecules are possibly due to the base activity of the cationic choline group. Peak tailing effects from cholinergic compounds are nearly nonexistent and retained well by the TOCO phase.

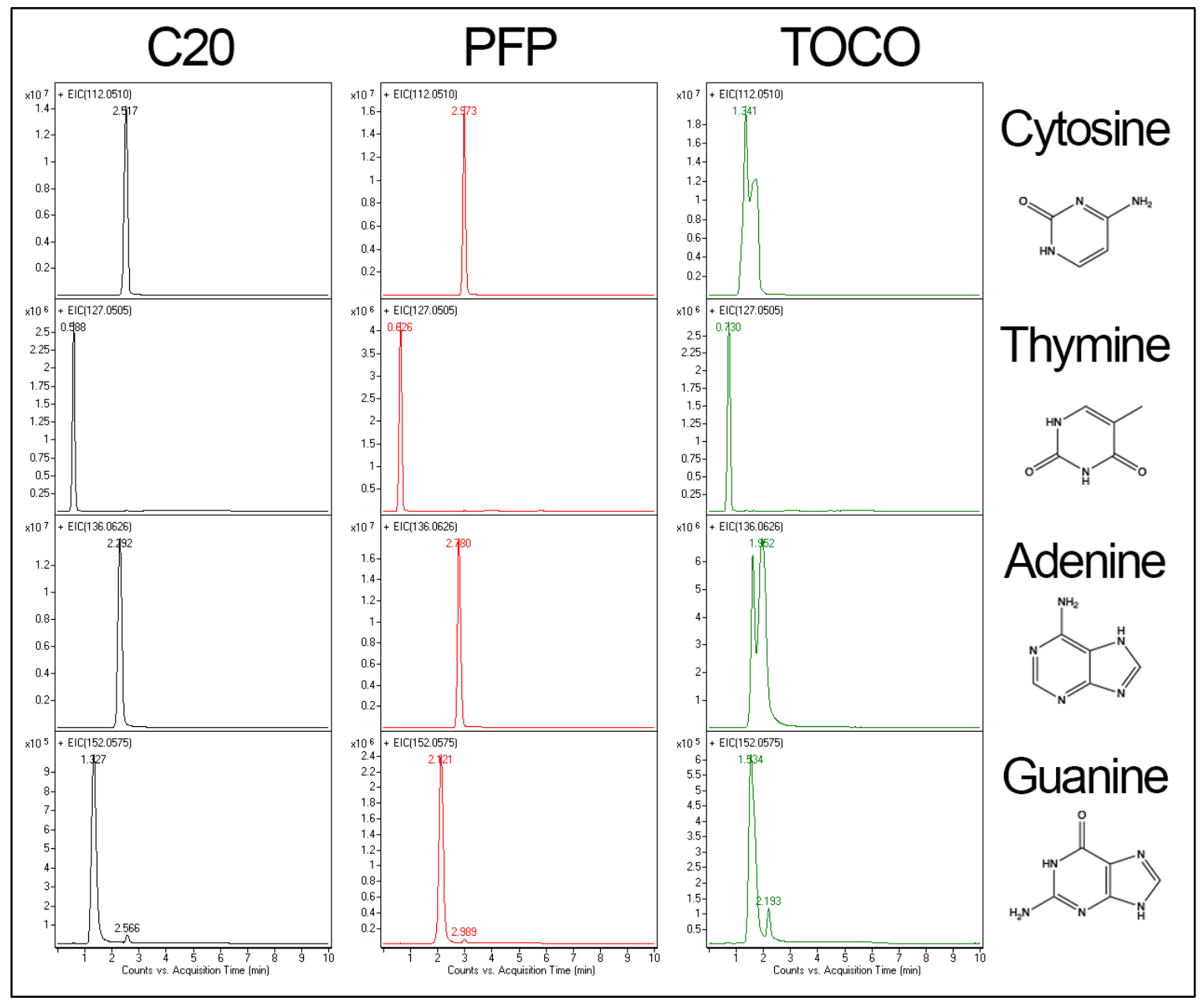

Figure 98. ANP Retention of Four Nucleobases. 
Figure 98 shows four highly polar nucleobases tested on the new columns under ANP condition. Some levels of retention were achieved for three nucleobases excluding thymine which is a structural analogue of uracil. The PFP column has achieved the longest retention followed by the $\mathrm{C} 20$ column. Once again, the TOCO phase produced poorly retained split peaks.

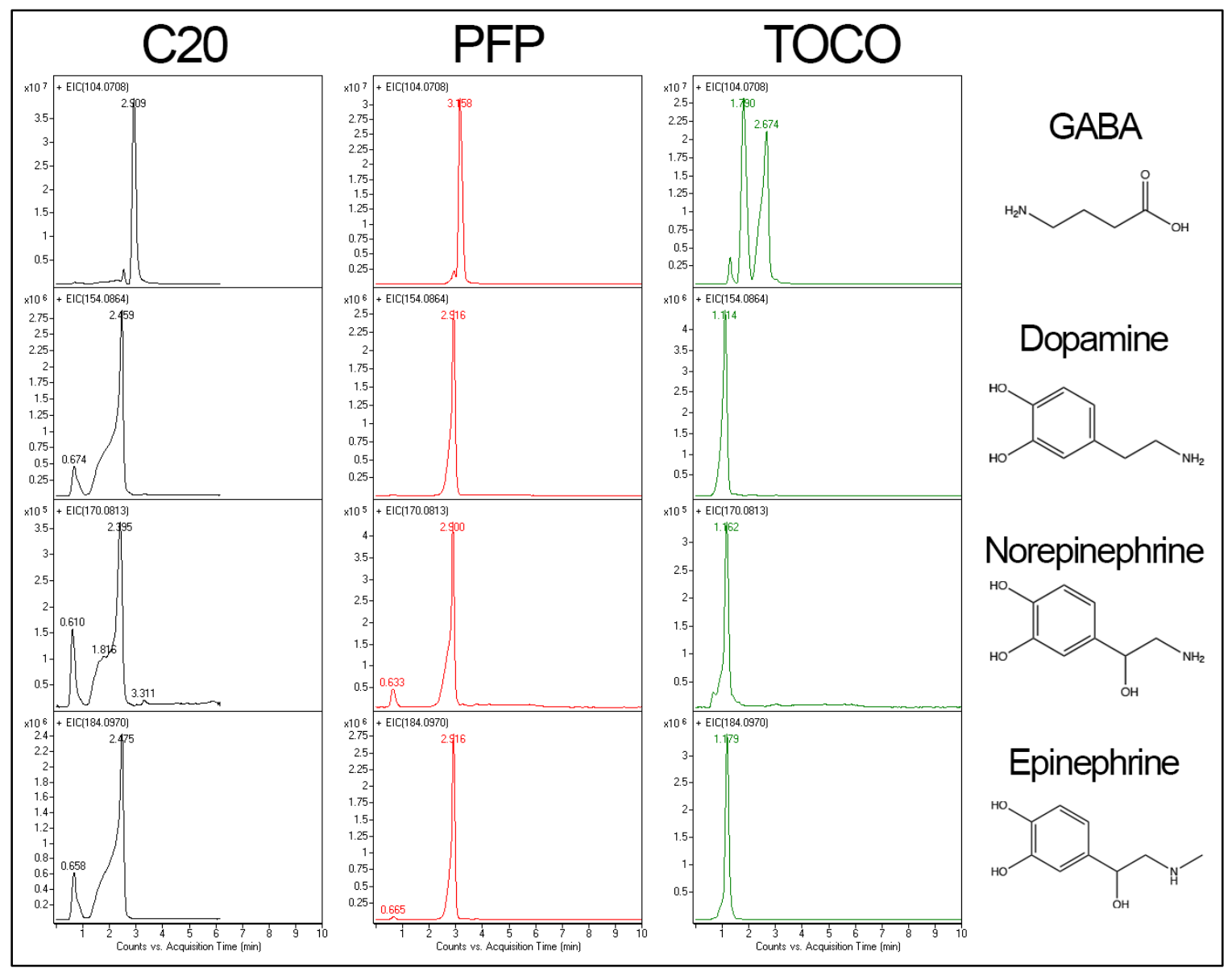

Figure 99. ANP Retention of Four Neurotransmitters.

Neurotransmitters were not only difficult to separate but also to obtain decently shaped peaks (Figure 99). The TOCO phase was the only column unable to achieve the analyte retention. Peak fronting was continuously observed for the $\mathrm{C} 20$ column except for GABA. Based on the peak shape and intensity (ion count $>1 \times 10^{7}$ ), peak distortion 
is likely due to sample overloading which can be improved by reducing the analyte concentration. The PFP column was able to produce symmetric peaks with very slight peak fronting.

The retention of water-soluble vitamins is very likely achievable through the ANP mode, but the RP mode is also effective if a column has strong RP characteristics. Figure 100 shows the retention of three vitamins under RP conditions. While C20 achieves very minimal separation, all three vitamins were retained for more than 2 minutes with the gradient method used. For ANP retention shown in Figure 101, the method was effective for pyridoxine and thiamine for all three columns; however, riboflavin and folic acid were only slightly retained by the TOCO phase.

Figure 102 shows ANP retention of sugar molecules. Using the designed gradient, the TOCO phase was the only column capable of retaining the molecules. The retention trend observed here is generally related to the length of sugar chain: monomer $<$ dimer $<$ trimer. The first three monosaccharides were barely retained for about 0.89 to $1.20 \mathrm{~min}$. Disaccharides were retained for more than $1.90 \mathrm{~min}$ and all five were partially separated by about $0.1 \mathrm{~min}$ or more. Lastly, both trisaccharides were retained for over 2.6 minutes with 0.07 mins difference in retention time. 


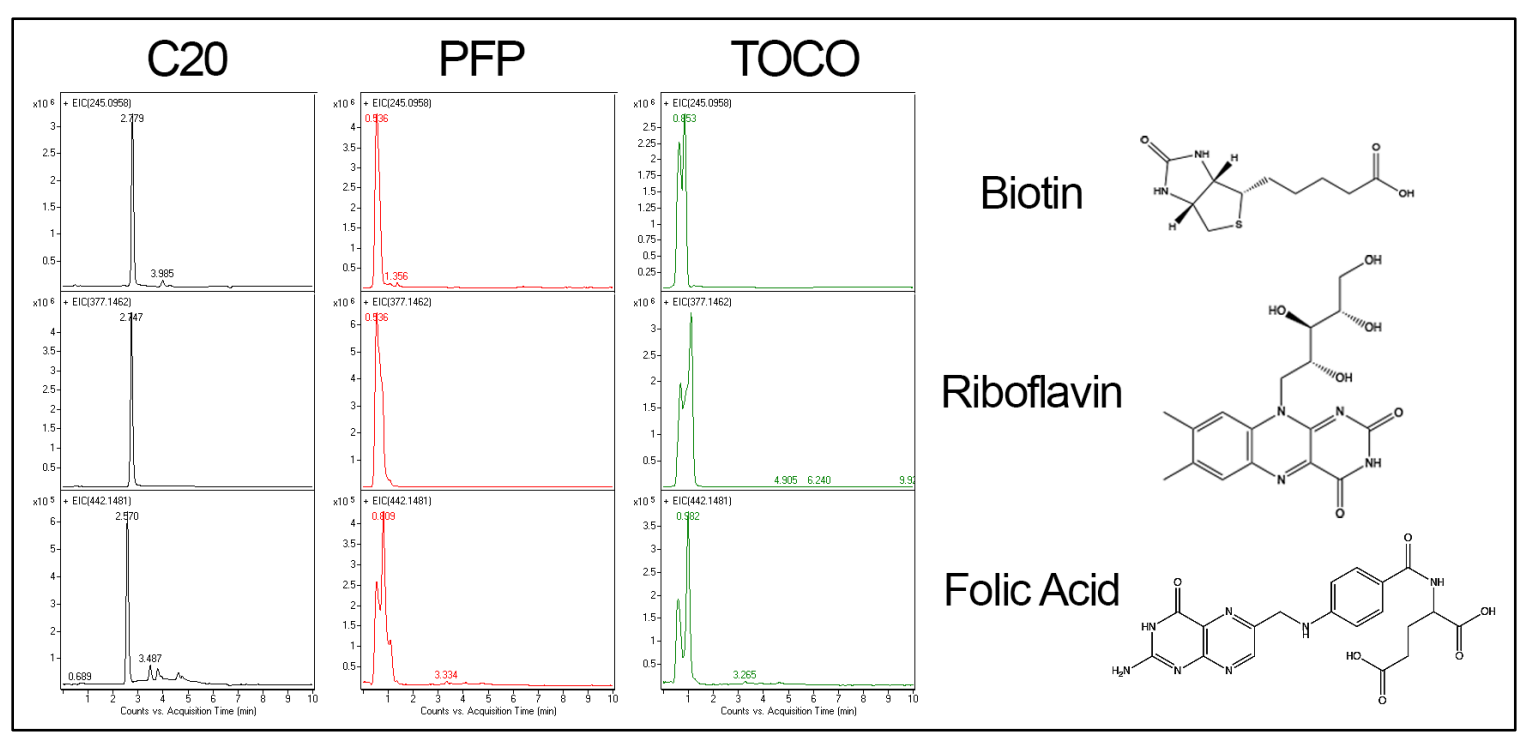

Figure 100. RP Retention of Three Vitamins.

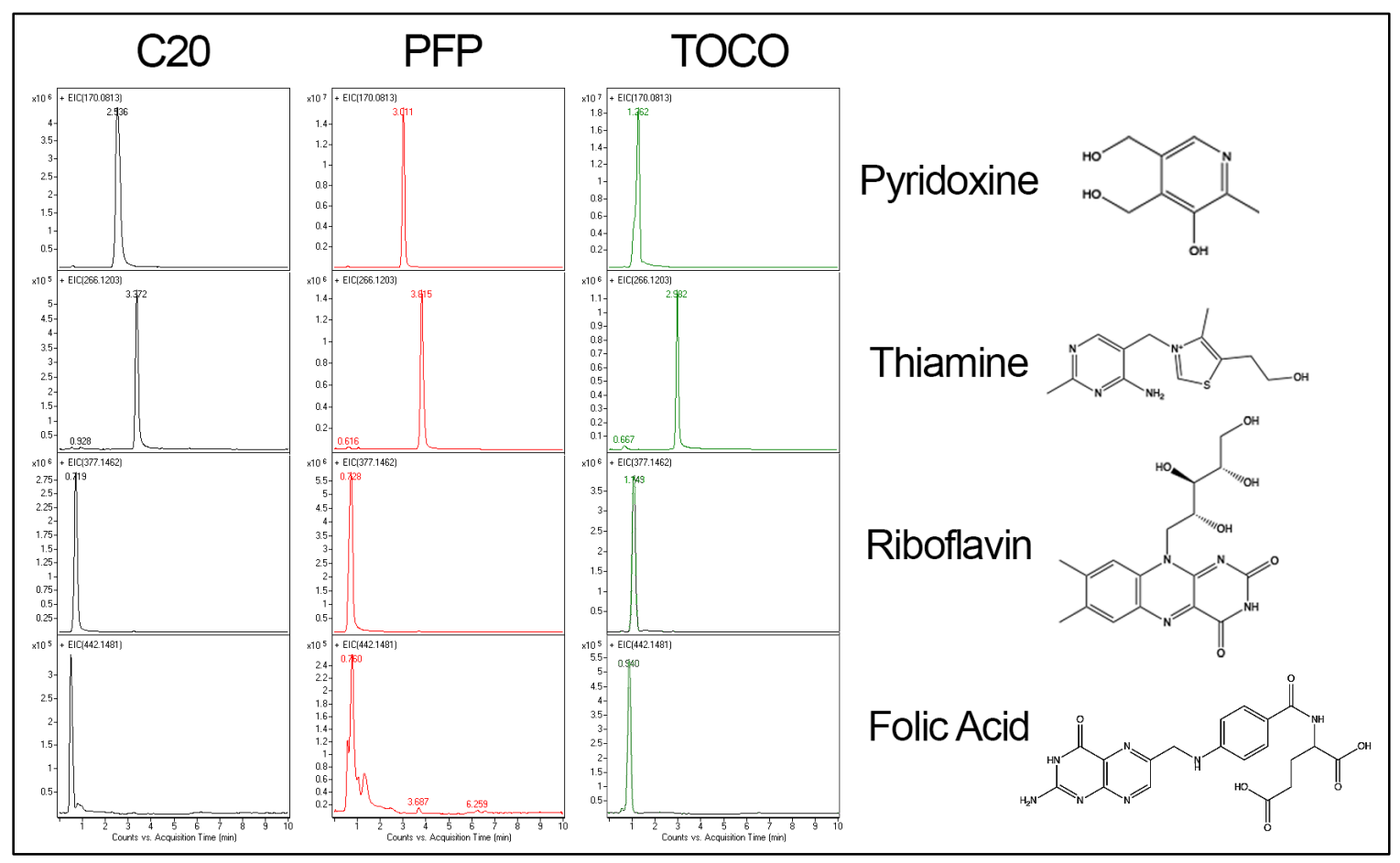

Figure 101. ANP Retention of Four Vitamins. 


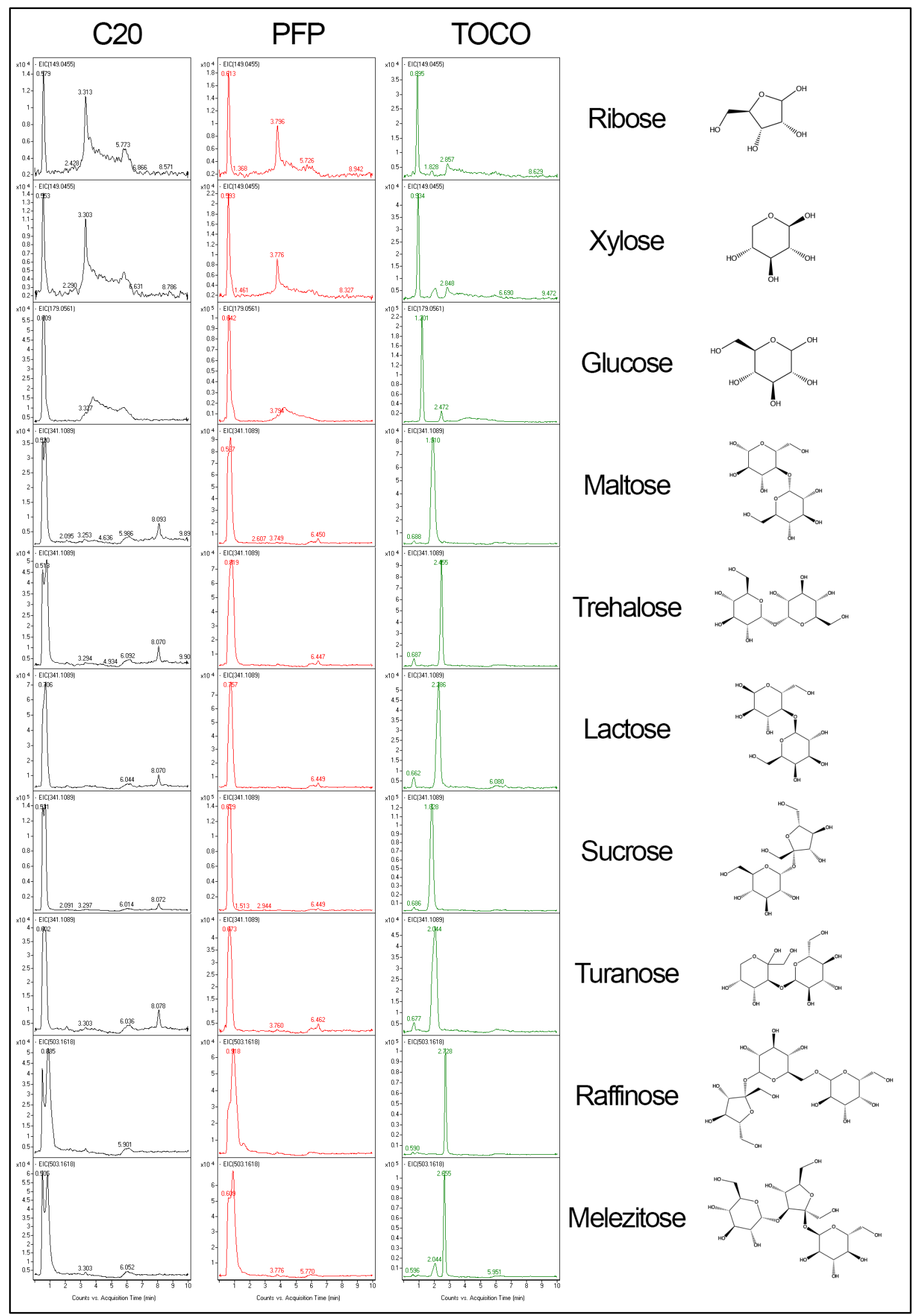

Figure 102. ANP Retention of Mono-, Di-, and Tri- Saccharides. 


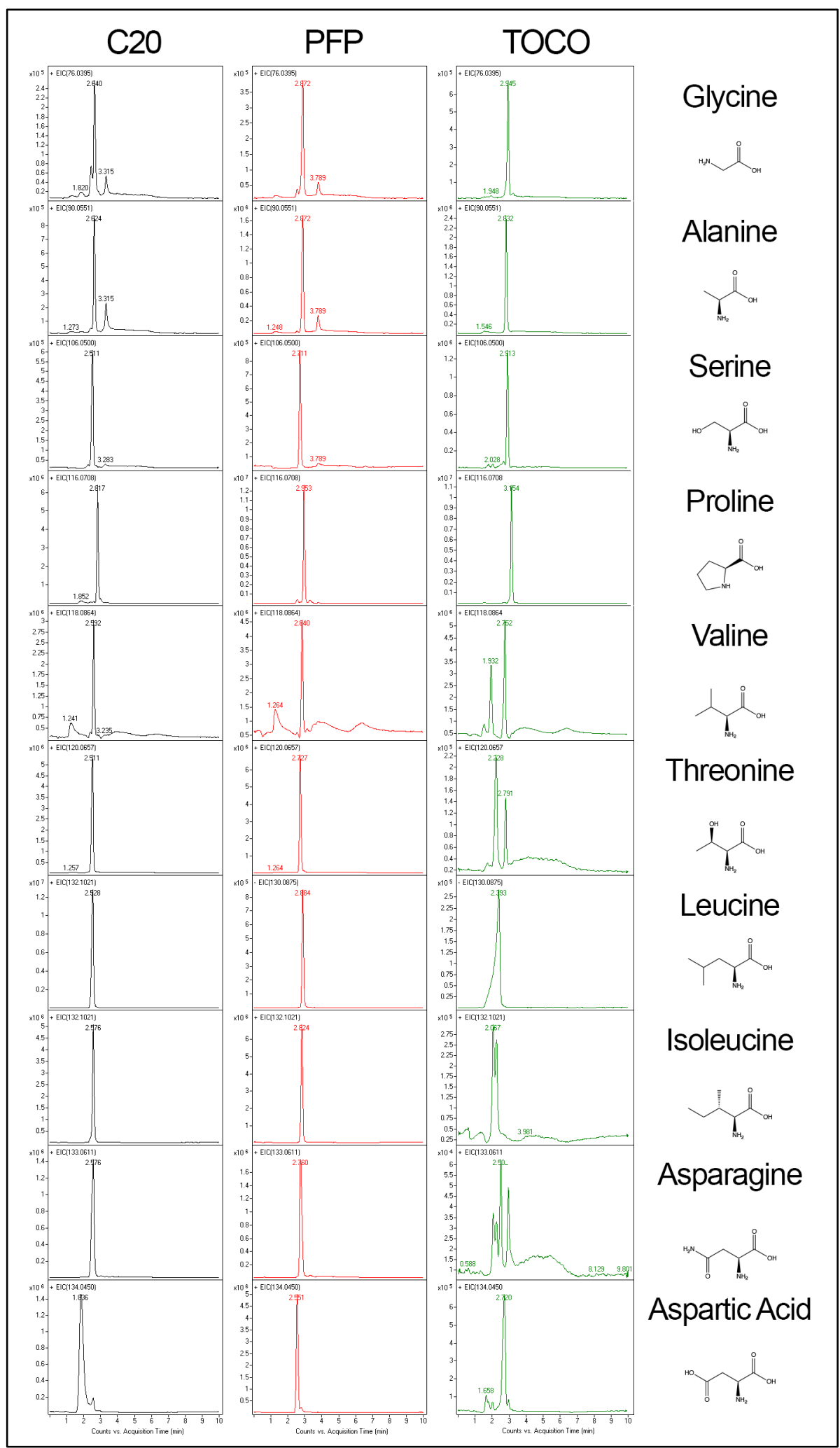

Figure 103. ANP Retention of Nineteen Amino Acids (1/2). 


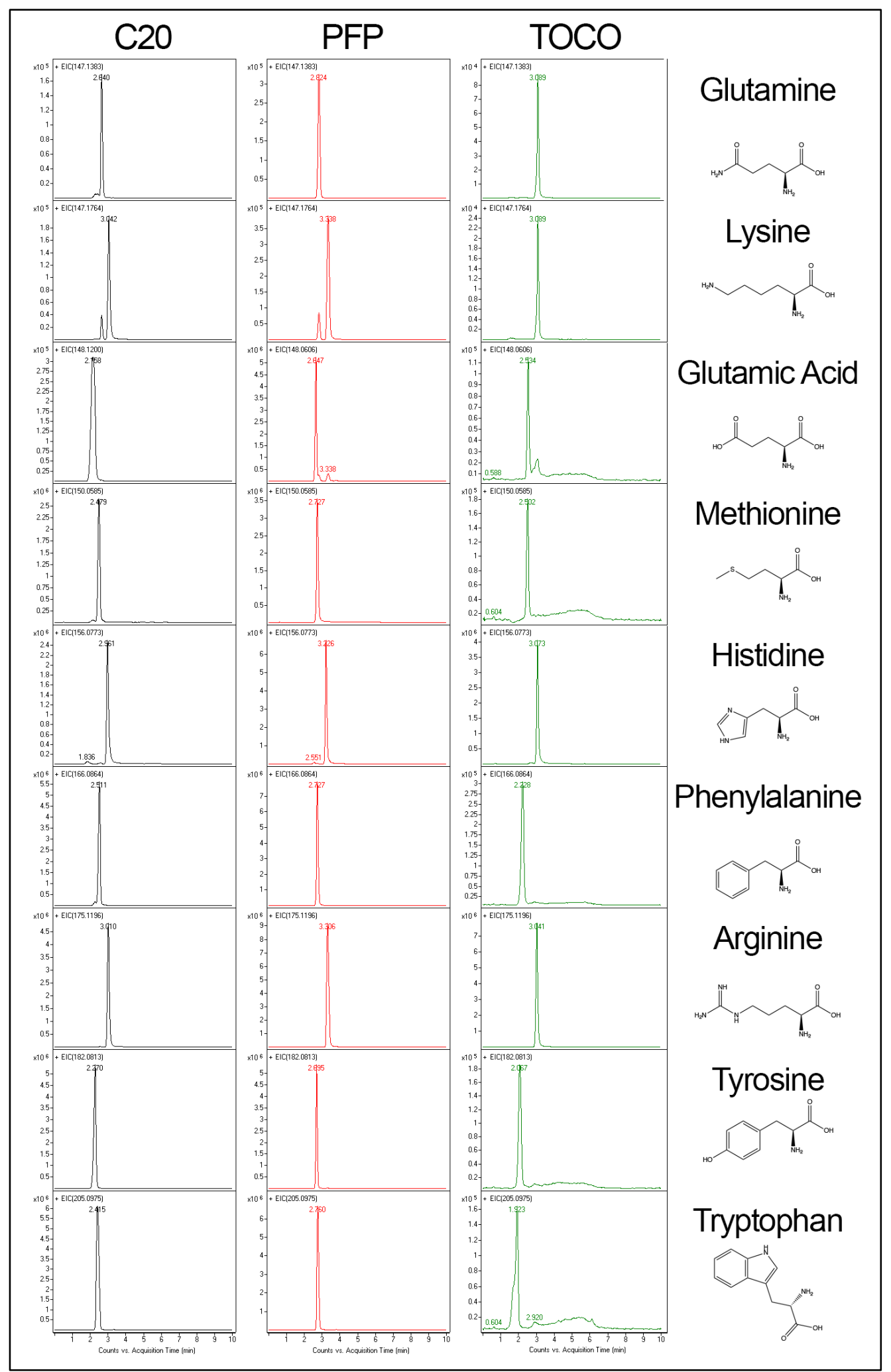

Figure 104. ANP Retention of Nineteen Amino Acids (2/2). 
Finally, the ANP retention of nineteen amino acids were demonstrated; cysteine was neglected from the selectivity study due to its poor ionization efficiency. Overall, amino acid retention was possible on all three columns (Figure 103,104) with retention time ranging from $1.8-3.3$ minutes. The $\mathrm{C} 20$ and PFP columns were able to consistently produce sharp and symmetric peaks. On the other hand, the TOCO phase occasionally had severely distorted or split peaks (for: valine, threonine, leucine, isoleucine, and asparagine). All amino acid residues are retained the longest on the PFP column except for alanine, proline, serine, aspartic acid and glutamine which were retained longer on the TOCO column.

\section{CONCLUSION}

The objective of the study to synthesize novel silica hydride stationary phases (TOCO, PFP, C20, LEU) by catalytic hydrosilylation was successfully achieved. The DRIFT spectra of reaction products were consistent with expected product spectra, based on the reference ATR spectra collected from starting olefin materials. ${ }^{13} \mathrm{C} \mathrm{CP}-$ MAS solid-state NMR spectra contained all predicted peaks for carbons directly bonded to hydrogen(s); however, spectra for LEU cannot be interpreted due to severe peak broadening effect. Elemental carbon analysis results and the Berendsen - de Galan equation were used to calculate surface coverage by the moieties; coverage values ranged from 1.269 to $1.736 \mu \mathrm{mol} / \mathrm{m}^{2}$ (Table 23). Since the LEU phase was the most recently developed column, it was excluded from extensive performance studies. 
Table 23. Summary of Four Column Properties.

\begin{tabular}{|c|c|c|c|c|}
\hline Criteria & C20 & PFP & TOCO & LEU \\
\hline \hline $\begin{array}{c}\text { Surface Coverage } \\
\left(\mu \mathrm{mol} / \mathrm{m}^{2}\right)\end{array}$ & 1.434 & 1.736 & 1.269 & 1.286 \\
\hline Column Spec. & \multicolumn{3}{|c|}{$2.1 \times 75 \mathrm{~mm}, 100 \AA, 4 \mu \mathrm{m}$} \\
\hline Silanol Activity & \multicolumn{4}{|c|}{ Low } \\
\hline $\begin{array}{c}\text { Metal Activity } \\
\text { Hydrophobicity } \\
\text { Parameter }\end{array}$ & 0.0354 & 0.0290 & 0.0224 & N/A \\
\hline $\begin{array}{c}\text { Dual Retention } \\
\text { Capability }\end{array}$ & \multicolumn{3}{|c|}{ Yes } \\
\hline
\end{tabular}

Table 24. Analyte Retention Time in Dual Retention Capability Test.

\begin{tabular}{|c|c|c|c|c|c|c|c|c|c|c|c|c|}
\hline \multirow{2}{*}{$\% \mathrm{~B}$} & \multicolumn{4}{|c|}{ Adenine/Adenosine } & \multicolumn{4}{|c|}{ Caffeine } & \multicolumn{4}{|c|}{ Glyburide } \\
\hline & $* \mathrm{C}$ & $* \mathrm{P}$ & $\mathrm{T}$ & $\mathrm{L}$ & $\mathrm{C}$ & $\mathrm{P}$ & $\mathrm{T}$ & $\mathrm{L}$ & $\mathrm{C}$ & $\mathrm{P}$ & $\mathrm{T}$ & $\mathrm{L}$ \\
\hline 100 & $* *_{-}$ & $* *_{-}$ & $* *_{-}$ & $* *_{-}$ & 1.9 & 1.6 & 2.0 & 1.9 & 0.6 & 0.6 & 0.7 & 0.6 \\
\hline 90 & 2.6 & 3.1 & 1.4 & 2.3 & 0.9 & 0.8 & 0.9 & 0.8 & 0.6 & 0.6 & 0.6 & 0.5 \\
\hline 80 & 1.2 & 1.4 & 0.8 & 1.1 & 0.8 & 0.8 & 0.8 & 0.7 & 0.5 & 0.6 & 0.6 & 0.5 \\
\hline 70 & 0.9 & 1.0 & 0.7 & 0.8 & 0.7 & 0.7 & 0.8 & 0.7 & 0.7 & 0.7 & 0.6 & 0.6 \\
\hline 60 & 0.7 & 0.8 & 0.6 & 0.7 & 0.7 & 0.7 & 0.8 & 0.7 & 0.8 & 0.8 & 0.7 & 0.7 \\
\hline 50 & 0.7 & 0.7 & 0.6 & 0.6 & 0.8 & 0.8 & 0.8 & 0.7 & 1.1 & 1.0 & 0.8 & 0.9 \\
\hline 40 & 0.6 & 0.7 & 0.6 & 0.6 & 0.8 & 0.8 & 0.8 & 0.7 & 1.8 & 1.5 & 1.0 & 1.3 \\
\hline 30 & 0.6 & 0.7 & 0.6 & 0.6 & 0.8 & 1.0 & 0.9 & 0.9 & 4.5 & 2.8 & 1.6 & 2.5 \\
\hline 20 & 0.6 & 0.7 & 0.6 & 0.7 & 1.0 & 1.4 & 1.1 & 1.2 & $* *_{-}$ & 8.7 & 3.6 & 6.7 \\
\hline 10 & 0.7 & 0.7 & 0.7 & 0.8 & 1.7 & 3.1 & 2.0 & 2.3 & $* *_{-}$ & $* *_{-}$ & $* *_{-}$ & $* *_{-}$ \\
\hline \multicolumn{13}{|c|}{$\begin{array}{l}\mathrm{C}=\mathrm{C} 20 ; \mathrm{P}=\mathrm{PFP} ; \mathrm{T}=\mathrm{TOCO} ; \mathrm{L}=\mathrm{LEU} \\
* \text { - Adenine was used } \\
* * \text { - Over Retention }\end{array}$} \\
\hline
\end{tabular}

According to the QC test and the SRM870 evaluation results, all newly developed columns exhibited low silanol/metal activities. Based on both SRM870 and Neue tests, 
the order of highest to the lowest hydrophobicity of the three columns are as follows: C20 $>$ PFP $>$ TOCO. The retention of the three analyte molecules by all four novel stationary phases showed "U-shaped" profiles, which confirms the ANP/RP dualretention capability of the columns (Table 24).

A series of analyte selectivity tests provided better understanding of the column's general behavior and their potential applications. First, the $\mathrm{C} 20$ phase was shown to be the most reliable column for its consistency to produce reasonable retention and separation for analytes over a wide polarity range. Furthermore, the highest RP retentivity and selectivity for polyaromatic hydrocarbons and methylene groups were observed for the $\mathrm{C} 20$ column (Table 25).

Table 25. Analyte Retention Time in Evaluation Tests Under RP Condition.

\begin{tabular}{|c|c|c|c|c|c|}
\hline \multirow{2}{*}{$\begin{array}{c}\text { Test } \\
\text { Types }\end{array}$} & \multirow{2}{*}{ Analytes } & \multicolumn{4}{|c|}{ tR (min) } \\
\cline { 2 - 6 } & & $\mathbf{C 2 0}$ & PFP & TOCO & LEU \\
\hline \hline \multirow{3}{*}{ QC Test } & Uracil & 0.9 & 1.0 & 0.8 & 1.1 \\
\cline { 2 - 6 } & Pyridine & 0.9 & 1.0 & 1.0 & 1.3 \\
\cline { 2 - 6 } & Phenol & 5.7 & 3.6 & 4.0 & 4.1 \\
\hline \hline \multirow{4}{*}{ Neue Test } & Uracil & 2.1 & 2.2 & 2.3 & N/A \\
\cline { 2 - 6 } & Naphthalene & 10.5 & 5.9 & 4.0 & N/A \\
\cline { 2 - 6 } & Acenaphthene & 20.2 & 9.6 & 5.4 & N/A \\
\hline \hline \multirow{5}{*}{ SRM 870 } & Uracil & 1.1 & 1.4 & 1.4 & 1.0 \\
\cline { 2 - 6 } & Toluene & 1.9 & 1.4 & 1.4 & 1.2 \\
\cline { 2 - 6 } & Ethyl Benzene & 2.1 & 1.4 & 1.4 & 1.2 \\
\cline { 2 - 6 } & Quinizarin & 4.5 & 1.4 & 2.3 & 2.1 \\
\cline { 2 - 6 } & Amitriptyline & 20.0 & 7.1 & 6.5 & 11.7 \\
\hline \hline \multirow{4}{*}{ DMB Mix } & Aniline & 8.6 & 5.4 & 3.2 & 5.8 \\
\cline { 2 - 6 } & o-DMB & 8.1 & 6.1 & 3.5 & 5.5 \\
\cline { 2 - 6 } & p-DMB & 8.6 & 6.8 & 4.3 & 6.1 \\
\cline { 2 - 6 } & m-DMB & 8.9 & 7.1 & 4.6 & 6.3 \\
\hline
\end{tabular}


Table 26. Analyte Retention Time in ANP Selectivity Test (1/2).

\begin{tabular}{|c|c|c|c|c|}
\hline \multirow{2}{*}{ Groups } & \multirow{2}{*}{ Analytes } & \multicolumn{3}{|c|}{ tR (min) } \\
\hline & & $\mathrm{C20}$ & PFP & TOCO \\
\hline \multirow{8}{*}{$\begin{array}{l}\text { Nootropics } \\
\text { (ANP) }\end{array}$} & Aniracetam & 0.6 & 0.6 & 0.6 \\
\hline & Noopept & 0.6 & 0.6 & 0.6 \\
\hline & Phenylpiracetam & 0.7 & 0.7 & $*_{-}$ \\
\hline & Coluracetam & 0.8 & 0.7 & 0.8 \\
\hline & Fasoracetam & 0.8 & 0.8 & 1.0 \\
\hline & Pramiracetam & 2.3 & 2.6 & $*_{-}$ \\
\hline & Alpha-GPC & 2.8 & 2.8 & 3.6 \\
\hline & CDP-choline & 3.0 & 3.0 & 3.6 \\
\hline \multirow{4}{*}{$\begin{array}{l}\text { Vitamins } \\
\text { (ANP) }\end{array}$} & Folic Acid & 0.5 & $*_{-}$ & 0.9 \\
\hline & Riboflavin & 0.7 & 0.7 & 1.1 \\
\hline & Pyridoxine & 2.5 & 3.0 & 1.3 \\
\hline & Thiamine & 3.4 & 3.6 & 3.0 \\
\hline \multirow{4}{*}{$\begin{array}{l}\text { Nucleobases } \\
\text { (ANP) }\end{array}$} & Thymine & 0.6 & 0.6 & 0.7 \\
\hline & Guanine & 1.3 & 2.1 & 1.5 \\
\hline & Adenine & 2.3 & 2.8 & $*_{-}$ \\
\hline & Cytosine & 2.5 & 3.0 & $*_{-}$ \\
\hline \multirow{4}{*}{$\begin{array}{c}\text { Neurotransmitters } \\
\text { (ANP) }\end{array}$} & Norepinephrine & $*_{-}$ & 2.9 & 1.2 \\
\hline & Dopamine & $*_{-}$ & 2.9 & 1.1 \\
\hline & Epinephrine & $*_{-}$ & 2.9 & 1.2 \\
\hline & GABA & 2.9 & 3.2 & $*_{-}$ \\
\hline \multirow{10}{*}{$\begin{array}{l}\text { Sugars } \\
\text { (ANP) }\end{array}$} & Ribose & $* *_{-}$ & $* *_{-}$ & 0.9 \\
\hline & Xylose & $* *_{-}$ & $* *_{-}$ & 0.9 \\
\hline & Glucose & $* *_{-}$ & $* *_{-}$ & 1.2 \\
\hline & Sucrose & $* *_{-}$ & $* *_{-}$ & 1.8 \\
\hline & Maltose & $* *_{-}$ & $* *_{-}$ & 1.9 \\
\hline & Turanose & $* *_{-}$ & $* *_{-}$ & 2.0 \\
\hline & Lactose & $* *_{-}$ & $* *_{-}$ & 2.3 \\
\hline & Trehalose & $* *_{-}$ & $* *_{-}$ & 2.5 \\
\hline & Melezitose & $* *_{-}$ & $* *_{-}$ & 2.7 \\
\hline & Raffinose & $* *_{-}$ & $* *_{-}$ & 2.7 \\
\hline
\end{tabular}


Table 27. Analyte Retention Time in ANP Selectivity Test (2/2).

\begin{tabular}{|c|c|c|c|c|}
\hline \multirow{2}{*}{ Groups } & \multirow{2}{*}{ Analytes } & \multicolumn{3}{|c|}{ tR (min) } \\
\hline & & $\mathrm{C20}$ & PFP & TOCO \\
\hline \multirow{19}{*}{$\begin{array}{l}\text { Amino Acids } \\
\text { (ANP) }\end{array}$} & Aspartic Acid & 1.8 & 2.6 & 2.7 \\
\hline & Glutamic Acid & 2.2 & 2.6 & 2.5 \\
\hline & Tyrosine & 2.3 & 2.7 & 2.1 \\
\hline & Tryptophan & 2.4 & 2.8 & 1.9 \\
\hline & Methionine & 2.5 & 2.7 & 2.5 \\
\hline & Serine & 2.5 & 2.7 & 2.9 \\
\hline & Threonine & 2.5 & 2.7 & $*_{-}$ \\
\hline & Phenylalanine & 2.5 & 2.7 & 2.2 \\
\hline & Leucine & 2.5 & 2.9 & 2.4 \\
\hline & Isoleucine & 2.6 & 2.8 & 2.1 \\
\hline & Asparagine & 2.6 & 2.8 & $*_{-}$ \\
\hline & Valine & 2.6 & 2.8 & $*_{-}$ \\
\hline & Alanine & 2.6 & 2.9 & 2.8 \\
\hline & Glycine & 2.6 & 2.9 & 2.9 \\
\hline & Glutamine & 2.6 & 2.8 & 3.1 \\
\hline & Proline & 2.8 & 3.0 & 3.2 \\
\hline & Histidine & 3.0 & 3.2 & 3.1 \\
\hline & Arginine & 3.0 & 3.3 & 3.0 \\
\hline & Lysine & 3.0 & 3.3 & 3.1 \\
\hline $\begin{array}{l}\text { * - Poor/Split } \\
\text { ** - No Retent }\end{array}$ & & & & \\
\hline
\end{tabular}

While polar molecules such as amino acids can also be retained by C20 under ANP conditions, the retention times were the shortest among the three columns (Table 26, 27). Second, the PFP column had the best regio-isomer selectivity for methoxybenzene analogues (Table 25). This feature is potentially useful for the separation of similar pharmaceutically active molecules that tend to contain benzene rings with polarizable functional groups. 
For example, nucleobases and neurotransmitters were retained remarkably well on the PFP column under ANP conditions (Table 26). In addition, aromatic compounds such as nootropic drug molecules are also very well-retained under RP condition (Table 28).

Table 28. Analyte Retention Time in RP Selectivity Test.

\begin{tabular}{|c|c|c|c|c|}
\hline \multirow{2}{*}{ Groups } & \multirow{2}{*}{ Analytes } & \multicolumn{3}{|c|}{ tR (min) } \\
\hline & & $\mathrm{C20}$ & PFP & TOCO \\
\hline \multirow{11}{*}{$\begin{array}{l}\text { Steroids } \\
\text { (RP) }\end{array}$} & Prednisolone & 8.4 & 7.0 & 6.5 \\
\hline & Adrenosterone & 9.6 & 8.2 & 7.5 \\
\hline & Corticosterone & 9.7 & 8.0 & 7.7 \\
\hline & $\beta$-Estradiol & 10.5 & 8.9 & 8.4 \\
\hline & Estrone & 10.7 & 9.2 & 8.6 \\
\hline & 17 $\alpha$-Ethynylestradiol & 10.8 & 9.2 & 8.5 \\
\hline & $\Delta 4$-Androsten-3,17-dione & 11.2 & 9.4 & 8.7 \\
\hline & Ethisterone & 11.4 & 9.4 & 8.7 \\
\hline & (-)-Norgestrel & 11.6 & 9.6 & 9.0 \\
\hline & $11 \alpha$-acetoxyprogesterone & 11.7 & 9.9 & 9.1 \\
\hline & Progesterone & 13.2 & 10.5 & 9.8 \\
\hline \multirow{8}{*}{$\begin{array}{l}\text { Nootropics } \\
\text { (RP) }\end{array}$} & "Alpha-GPC & 0.5 & 0.6 & 0.8 \\
\hline & CDP-choline & 0.6 & 0.6 & 0.8 \\
\hline & Pramiracetam & 0.8 & 1.1 & $*_{-}$ \\
\hline & Fasoracetam & 1.3 & 1.4 & 1.5 \\
\hline & Phenylpiracetam & 2.0 & 2.1 & 1.4 \\
\hline & Aniracetam & 2.9 & 3.0 & 2.0 \\
\hline & Noopept & 3.0 & 2.9 & 2.2 \\
\hline & Coluracetam & 3.3 & 3.4 & 3.1 \\
\hline \multirow{3}{*}{$\begin{array}{l}\text { Vitamins } \\
\text { (RP) }\end{array}$} & Folic Acid & 2.6 & $*_{-}$ & $*_{-}$ \\
\hline & Riboflavin & 2.7 & 0.5 & $*_{-}$ \\
\hline & Biotin & 2.8 & 0.5 & $*_{-}$ \\
\hline
\end{tabular}


Table 29. RP Analyte Retention Profile in Bioanalytical Projects.

\begin{tabular}{|c|c|c|c|c|}
\hline \multirow{2}{*}{ Projects } & \multirow{2}{*}{ Analytes } & \multicolumn{3}{|c|}{ tR (min) } \\
\hline & & $\mathrm{C} 20$ & PFP & TOCO \\
\hline Cannabinoid & CBD & 6.6 & 5.9 & 5.3 \\
\hline \multirow{13}{*}{$\begin{array}{l}\text { Drug Analysis } \\
\text { (Human } \\
\text { Urine) }\end{array}$} & D-amphetamine & 4 & 1.9 & 1.2 \\
\hline & D-methamphetamine & $*_{-}$ & $*_{-}$ & $*_{-}$ \\
\hline & Phentermine & $*_{-}$ & $*_{-}$ & $*_{-}$ \\
\hline & Ephedrine & $*_{-}$ & $*_{-}$ & $*_{-}$ \\
\hline & Pseudoephedrine & $*_{-}$ & $*_{-}$ & $*_{-}$ \\
\hline & MDA & 4 & 1.9 & 1.2 \\
\hline & MDMA & 7 & 4.1 & 1.7 \\
\hline & Nordiazepam & 8.1 & 4.9 & 3.7 \\
\hline & Diazepam & 10.5 & 9.4 & 8.9 \\
\hline & Temazepam & 10 & 9.1 & 8.8 \\
\hline & Alpha-hydroxyalprazolam & 10.1 & 9 & 8.8 \\
\hline & THCA & $* *_{-}$ & $* *_{-}$ & $* *_{-}$ \\
\hline & Morphine-3-glucuronide & 9.3 & 8.8 & 8.7 \\
\hline \multirow{8}{*}{$\begin{array}{l}\text { Drug Analysis } \\
\text { (Standards) }\end{array}$} & $(\mathrm{R} / \mathrm{S})$-Cathinone & 2.3 & 1.6 & 0.9 \\
\hline & 4-fluromethcathinone & 3.9 & 2.3 & 1.1 \\
\hline & N-ethylcathinone & 5 & 2.9 & 1.5 \\
\hline & MDMA & 7 & 4 & 1.6 \\
\hline & Mephedrone & 7.2 & 4.5 & 1.7 \\
\hline & Pentedrone & 7.8 & 5.3 & 2 \\
\hline & Heroin & 8.4 & 8.2 & 5.2 \\
\hline & LSD & 9.1 & 9.1 & 8.8 \\
\hline \multirow{5}{*}{ Grape Skin } & Catechin & 3.6 & 2.6 & 1.8 \\
\hline & Epicatechin & 3.9 & 2.9 & 2.4 \\
\hline & Quercetin-3-O-glucoside & 4.8 & 4.5 & 4.2 \\
\hline & Kaempeferol-3-O-glucoside & 5.2 & 4.7 & 4.4 \\
\hline & Quercetin-3-O-glucoside & 6.2 & 5.8 & 5.2 \\
\hline
\end{tabular}


Third, the TOCO phase was by far the most unique column characterized in this study. Retentivity on TOCO is considerably weaker than the other two columns, but it seems to interact strongly with a few selective compounds. Based on the trends observed in the selectivity test, the TOCO column generally has stronger selectivity for larger and complex analyte molecules such as polycyclic aromatic and heteroaromatic compounds. For example, catechin and epicatechin were completely separated in a shorter period compared to the C20 column (Table 29). Also, it is visibly apparent that alpha-GPC and CDP-choline were most efficiently retained under ANP conditions by the TOCO column.

Most importantly, the TOCO phase was the only column capable of retaining and show some levels of separation for all disaccharides and trisaccharides (Table 26). The retention order observed for the sugar molecules was also based on their unit sizes; thus, the TOCO column can be potentially useful for the separation of larger sugar oligomers and glycosylated biopolymers. It is also worth noting that the TOCO column generally produces better analyte peak shape (e.g., amitriptyline). Split peaks occasionally observed for small analyte molecules (e.g., amino acids) are very likely concentrationdependent; thus, peak shape was significantly improved after 10 to 100 -fold sample dilution and comparable to the other two columns (not shown).

The novel silica hydride columns were tested for their potential applicability and effectiveness in demonstration research projects. The retention of selected phytocannabinoid was easily accomplished by all three columns. The C20 column seems to be the most effective column for the retention of most drug molecules in the 
human urine sample. Both PFP and TOCO were less retentive but capable of isolating specific drug classes such as benzodiazepines (Table 28). This feature can be useful even in HPLC coupled to less selective detectors such as UV or fluorescence.

For the grape metabolomics project, the dual retention capability of silica hydride materials can be beneficial in discovery-based studies where there is/are no targetedanalyte compound(s). In this study, at least ten statistically significant $(p<0.05)$ metabolomes were detected with reasonable signal intensity and peak shape. With further method optimization, more peak groups can be potentially generated and identified using metabolite databases.

In ANP-HDX-MS project, the study has shown that BE during the elution step is most significantly affected by the aqueous/organic composition of the mobile phase. Furthermore, ANP was demonstrated to be an effective alternative to RP for peptide retention and separation at reduced protiated solvent usage. In summary, the BE observed from an ANP gradient is much less than RP to a statistically significant level $(\mathrm{p}<0.05)$. At equal water volume usage, ANP gradient has reduced BE at least by $12 \%$ from an RP gradient method even when ANP retention was longer by 0.42 minutes. At equal retention time, the ANP gradient method that consumes $10 \%$ less water has reduced $\mathrm{BE}$ by at least $15 \%$.

Future investigation includes collaboration research with Dr. Ningkun Wang's group to utilize ANP-HDX-MS approach for the structural elucidation of human SIRT1 protein. In addition, the low temperature effect on ANP-HDX will be assessed. Other interesting studies could be further performance evaluation of the columns. For 
example, the LEU column is planned to be evaluated for chiral chromatography application. Furthermore, TOCO can be tested for selectivity of larger analyte molecules. Lastly, C20 and PFP columns can be compared to commercial C18 and phenyl/PFP columns to understand their differences in chromatographic properties. 


\section{REFERENCES}

[1] Tswett, M. S., Труды Варшавского общества естествоиспытателей, отделении биологии, 1905, 14 (6), 20-39.

[2] Moldoveanu, S. C.; David, V. Essentials In Modern HPLC Separations, Elsevier, MA, USA, 2013.

[3] Karger, B. L., J. Chem. Educ. 1997, 74 (1), 45.

[4] Martin, J. P.; Synge, R. L. M. Biochem. J. 1941, 35.

[5] Wicke, E. J. C. Giddings: Dynamics of Chromatography. Part. I: Principles and Theory. Marcel Dekker, NY, 1967, 71 (2), 236-236.

[6] Snyder, L.; Kirkland, J.; Dolan, J., Introduction to Modern Liquid Chromatography 3rd Ed. Wiley. 2010, 331-347.

[7] Dill, K. A. J. Phys. Chem. 1987, 91, 1980-19889.

[8] Vailaya, A.; Horváth, C. J. Chromatogr. A 1998, 829 (1-2), 1-27.

[9] Dorsey, J. G.; Dill, K. A. Chem. Rev. 1989, 89 (2), 331-346.

[10] J. Dolan. LCGC Europe 2008, 21, 5, 258-263.

[11] James, H.; Smith, N.W., Chromatography Today. 2012, 44-47.

[12] Brown, L.; Ciccone, B.; Pesek, J. J.; Matyska, M. T. Am. Lab. 2003, 23.

[13] Pesek, J. J.; Matyska, M. T. J. Sep. Sci. 2005, 28 (15), 1845-1854.

[14] Pesek, J. J.; Matyska, M. T. J. Sep. Sci. 2009, 32 (23-24), 3999-4011.

[15] Chu, C. H.; Jonsson, E.; Auvinen, M.; Pesek, J. J.; Sandoval, J. E.; Anal. Chem. 1993, 65, 808-816.

[16] Snyder, L. R.; Kirkland, J. J.; Glajch, J. L. Practical HPLC Method Development, 2nd ed.; Wiley: New York, NY, 1997.

[17] Nawrocki, J. Chromatographia 1991, 31 (3-4), 177-192.

[18] Nawrocki, J. Chromatographia 1991, 31 (3-4), 193-205. 
[19] Sandoval, J. E.; Pesek, J. J. Anal. Chem. 1989, 61 (18), 2067-2075.

[20] Wirth, M. J.; Fatunmbi, H. O. Anal. Chem. 1993, 65 (6), 822-826.

[21] Sandoval, J. E.; Pesek, J. J. Anal. Chem. 1991, 63 (22), 2634-2641.

[22] Pesek, J. J.; Matyska, M. T.; Sandoval, J. E.; Williamsen, E. J. J. Liq. Chromatogr. Relat. Technol. 1996, 19 (17-18), 2843-2865.

[23] Cobb, K. A.; Dolnik, V.; Novotny, M. Anal. Chem. 1990, 62 (22), 2478-2483.

[24] Olah, G. A.; Molnár, Á. Hydrocarbon Chemistry 2nd ed., 2003, 322-324.

[25] Speier, J. L.; Webster, J. A.; Barnes, G. H. J. Am. Chem. Soc. 1957, 79, 974.

[26] Chalk A. J.; Harrod, J. F. J. Am. Chem. Soc. 1965, 87, 16.

[27] Harrod, J. F.; Chalk, A. J. J. Am. Chem. Soc. 1966, 88, 3491.

[28] Ojima, I. The Hydrosilylation Reaction. The Chemistry of Functional Groups, Wiley, 1989, 1479-1526.

[29] Chalk, A. J. Trans. N. Y. Acad. Sci. 1970, 32, 481.

[30] Benkesser, R.; Kang, J. J. Organomet. Chem., 1980, 185, C9.

[31] Akapo, S. O.; Dimandja, J. M.; Pesek, J. J.; Matyska, M. T. Chromatographia, 1996, 42, 141.

[32] Pesek, J. J.; Matyska, M. T.; Oliva, M.; Evanchic, M. J. Chromatogr. A, 1998, 818, 145.

[33] Pesek, J. J.; Matyska, M. T.; Muley, S. Chromatographia 2000, 52, 439.

[34] Soukup, J.; Jandera, P. J. Chromatogr. A 2014, 1374, 102-111.

[35] Greco, G.; Letzel, T. J. Chromatogr. Sci. 2013, 51 (7), 684-693.

[36] Pesek, J. J.; Matyska, M. T.; Natekar, H. J. Sep. Sci. 2016, 39 (6), 1050-1055.

[37] Kulsing, C.; Yang, Y.; Munera, C.; Tse, C.; Matyska, M. T.; Pesek, J. J.; Boysen, R. I.; Hearn, M. T. W. Anal. Chim. Acta 2014, 817, 48-60. 
[38] Kulsing, C.; Nolvachai, Y.; Marriott, P. J.; Boysen, R. I.; Matyska, M. T.; Pesek, J. J.; Hearn, M. T. W.. J. Phys. Chem. B 2015, 119 (7), 3063-3069.

[39] Gray-Weale, A.; Beattie, J. K. Phys. Chem. Chem. Phys. 2009, 11 (46), 10994.

[40] Boysen, R. I.; Yang, Y.; Chowdhury, J.; Matyska, M. T.; Pesek, J. J.; Hearn, M. T. W. J. Chromatogr. A 2011, 1218 (44), 8021-8026.

[41] Bell, D. S.; Cramer, H. M.; Jones, A. D. J. Chromatogr. A. 2005, 1095 (1-2), 113118.

[42] Petruczynik, A.; Wróblewski, K.; Dzioba, K.; Waksmundzka-Hajnos, M. Open Chem. 2015, 13 (1).

[43] Gilar, M.; Yu, Y.-Q.; Ahn, J.; Fournier, J.; Gebler, J. C. J. Chromatogr. A 2008, $1191(1-2), 162-170$.

[44] Zhang, K.; Liu, X. J. Pharm. Biomed. Anal. 2016, 128, 73-88.

[45] Bell, D. S.; Jones, A. D. J. Chromatogr. A. 2005, 1073 (1-2), 99-109.

[46] Azzi, A.; Meydani, S. N.; Meydani, M.; Zingg, J. M. Arch. Biochem. Biophys. 2016, 595, 100-108.

[47] Azzi, A. Mol. Aspects Med. 2018, 61.

[48] Rimbach, G.; Moehring, J.; Huebbe, P.; Lodge, J. K. Molecules 2010, 15 (3), 1746-1761.

[49] Pullman, B.; Jortner, J. Eur. J. Biochem., 1982, 123, 473-475.

[50] Kuchan, M. J.; Moulton, C. J.; Dyer, R. A.; Jensen, S. K.; Schimpf, K. J.; Innis, S. M. Curr. Dev. Nutr. 2018, 2 (8).

[51] Wilburn, E. E.; Mahan, D. C.; Hill, D. A.; Shipp, T. E.; Yang, H. J. Anim. Sci. 2008, 86 (3), 584-591.

[52] Yarabe, H. H.; Shamsi, S. A.; Warner, I. M. Anal. Chem. 1999, 71 (18), 39923999.

[53] Billiot, E.; Macossay, J.; Thibodeaux, S.; Shamsi, S. A.; Warner, I. M. Anal. Chem. 1998, 70 (7), 1375-1381. 
[54] Neue, U. D.; Van Tran, K.; Iraneta, P. C.; Alden, B. A. J. Sep. Sci. 2003, 26 (3-4), 174-186.

[55] Vanommeslaeghe, K.; Guvench, O.; MacKerell A. D. Curr Pharm Des. 2014 ; 20(20): 3281-3292.

[56] Halgren, T. A. J. Comput. Chem. 1996, 17 (5-6), 490-519.

[57] Lewis, L. N.; Lewis, N. J. Am. Chem. Soc. 1986, 108, 7228.

[58] Benvenuto, K.; Botch-Jones, S.R.; Mallet, C. R. J Forensic Sci Criminol. 2017, 5(5): 502

[59] Pesek J. J., Matyska M. T., Kim A. M., J. Sep. Sci. 2013, 36 (17), 2760-2766.

[60] Melamud, E.; Vastag, L.; Rabinowitz, J. D. Anal. Chem. 2010, 82 (23), 9818 9826.

[61] Gu, Z.; Eils, R.; Schlesner, M. Bioinformatics 2016, 32 (18), 2847-2849.

[62] Wang, H.; Rempel, D. L.; Giblin, D.; Frieden, C.; Gross, M. L. Anal. Chem. 2017, $89(20), 10687-10695$.

[63] Das, T. K. Biophys. Methods Biother.; John Wiley \& Sons, Inc.: Hoboken, New Jersey, 2014, 381.

[64] Buxbaum, E. Biophysical Chemistry of Proteins: An Introduction to Laboratory Methods; Springer US: New York, NY, 2011.

[65] Kipping, M.; Schierhorn, A. J. Mass Spectrom. 2003, 38 (3), 271-276.

[66] Guttman, M.; Lee, K. K. Methods Enzymol.; Elsevier, 2016; 566, 405-426.

[67] Zhang, Y.; Fonslow, B. R.; Shan, B.; Baek, M.-C.; Yates, J. R. Chem. Rev. 2013, 113 (4), 2343-2394.

[68] Pesek, J. J.; Tang, V. H. Chromatographia 1994, 39 (11-12), 649-654.

[69] Matyska, M. T.; Pesek, J. J.; Grandhi, V. J. Sep. Sci. 2002, 25 (12), 741-748.

[70] G.E. Beredensen, L. de Galan, J. Liq. Chromatogr. 1978, 561.

[71] Sandoval, J. E. J. Chromatogr. A 1999, 852 (2), 375-381. 
[72] Akitt, J. W.; Mann, B. E. NMR and Chemistry: An Introduction to Modern NMR Spectroscopy, 4th ed.; S. Thornes: Cheltenham, U.K, 2000.

[73] Duer, M. J. Solid-State NMR Spectroscopy Principles and Applications; Blackwell Science Ltd: Oxford, UK, 2001.

[74] Slitcher, C. P. Principles of Magnetic Resonance, $3^{\text {rd }}$ ed.; Springer-Verlag: Berlin, Germany, 1989, 244.

[75] Fukushima, E.; Roeder, S. B. W. Experimental Pulse NMR: A Nuts and Bolts Approach; Westview Press: Boulder, CO, 2004.

[76] Kazakevich, Y., LoBrutto, R., HPLC for Pharmaceutical Scientists; WileyInterscience: Hoboken, N.J, 2007.

[77] Mamyrin, B. A.; Karataev, V. I.; Shmikk, D. V.; Zagulin, V. A. J. Exp. Theor. Phys., 1973, 64, 37, 1.

[78] Grabowsky, S.; Beckmann, J.; Luger, P. Aust. J. Chem. 2012, 65 (7), 785.

[79] Pesek, J.; Cash, T. A. Chromatographia 1989, 27 (11-12), 559-564.

[80] Pesek, J. J.; Matyska, M. T.; Prajapati, K. V. J. Sep. Sci. 2010, 33 (19).

[81] Bayer, E.; Albert, K.; Reiners, J.; Nieder, M.; Müller, D. J. Chromatogr. A, 1983 264, 197-213.

[82] Pesek, J. J.; Matyska, M. T.; Yu, R. J. J. Chromatogr. A 2002, 947 (2), 195-203.

[83] Pisanti, S.; Malfitano, A. M.; Ciaglia, E.; Lamberti, A.; Ranieri, R.; Cuomo, G.; Abate, M.; Faggiana, G.; Proto, M. C.; Fiore, D. Pharmacol Ther. 2017, 175, 133150 .

[84] Moreno-Sanz, G. Cannabis Cannabinoid Res. 2016, 1, 124-130.

[85] Perrotin-Brunel, H.; Buijs, W.; Van Spronsen, J.; Van Roosmalen, M. J.; Peters, C. J.; Verpoorte, R.; Witkamp, G. J. J. Mol. Struct. 2011, 987, 67-73.

[86] Watanabe, S.; Santos, T. Q.; Matyska-Pesek, M. T.; Pesek, J. J. J. Sep. Sci. 2019, 42, 1972-1979.

[87] Verpoorte, R., Alfermann, A. W., Metabolic Engineering of Plant Secondary Metabolism; Kluwer Academic Publishers: Dordrecht, 2000. 
[88] Xie, D.-Y. Plant Physiol. 2004, 134 (3), 979-994.

[89] Kanehisa, M.; Sato, Y.; Kawashima, M.; Furumichi, M.; Tanabe, M. Nucleic Acids Res. 2016, 44 (D1), D457-D462.

[90] Kanehisa, M.; Goto, S. Nucleic Acids Res. 2017, 45 (D1), D353-D361.

[91] Keppel, T. R.; Jacques, M. E.; Young, R. W.; Ratzlaff, K. L.; Weis, D. D. J. Am. Soc. Mass Spectrom. 2011, 22 (8), 1472-1476. 